\title{
Neuronal mechanisms of the adaptation of conditional visuomotor behavior
}

\author{
D I S S E R T A T I O N \\ for the award of the degree \\ "Doctor rerum naturalium" \\ Division of Mathematics and Natural Science \\ of the Georg-August-Universität Göttingen
}

submitted by

Stephanie Westendorff

from Essen

Göttingen 2010 
Doctoral thesis committee:

Dr. Alexander Gail (Advisor, First Referee)

Sensorimotor Group, BCCN

Deutsches Primatenzentrum (DPZ)

Kellnerweg 4

37077 Göttingen

Prof. Dr. Stefan Treue (Second Referee)

Abt. Kognitive Neurowissenschaften

Deutsches Primatenzentrum (DPZ)

Kellnerweg 4

37077 Göttingen

Prof. Dr. Fred Wolf

Theoretical Neurophysics, BCCN

Max Planck Institute for Dynamics and Self-Organization

Bunsenstrasse 10

37073 Göttingen

External thesis advisor:

Prof. Dr. Julia Fischer

Abt. Kognitive Ethologie

Deutsches Primatenzentrum (DPZ)

Kellnerweg 4

37077 Göttingen

PD. Dr. Ralf Heinrich

Abt. Neurobiologie

Johann-Friedrich-Blumenbach-Institut für Zoologie und Anthropologie

Berliner Strasse 28

37073 Göttingen

Prof. Dr. med. Hansjörg Scherberger

Abt. Neurobiologie

Deutsches Primatenzentrum (DPZ)

Kellnerweg 4

37077 Göttingen

Date of submission of the thesis: 30 September, 2010

Date of disputation: 28 October, 2010 
I hereby declare that this thesis has been written independently and with no other sources and aids than quoted.

Göttingen, 30 September, 2010

Stephanie Westendorff 
"In theory, theory and practice are the same. In practice, theory and practice are different."

Larry Snyder, Cold Spring Harbor, 2010 


\section{Acknowledgements}

During the time it took to write my PhD-thesis I occupied the time and help of several people, more than I will be able to mention here.

First and foremost I thank Alexander Gail for the opportunity to write this thesis in his laboratory and especially for his supervision during the whole time. I'm really grateful for all the time he spent helping me and I always enjoyed working for him. Furthermore I thank Stefan Treue for his advice and constructive feedback and Fred Wolf for his support as member of my thesis committee.

Julia Fischer, Ralf Heinrich and Hans Scherberger kindly agreed to evaluate my thesis, for which I'm also very thankful.

I'm very happy to also thank Sina Plümer for all her help in endless things during my time in this laboratory. Not only I could always rely on her help for problems concerning my work, but she also helped me in a lot of other things (e.g. driving me to the doctor and physiotherapist while I was on crutches for several weeks).

For help regarding the electrophysiological recordings and the monkeys I owe special thanks to Leonore Burchard, Dirk Prüße and Ludwig Ehrenreich.

I'm grateful for the help of Sabine Stuber and Beatrix Glaser regarding administrative questions and for the help of Ralf Brockhausen and Kevin Windolph regarding technical and computer questions.

Daniel, Steffen, Laura, Anja, Valeska, Ina, Sonia, Katharina, Pinar, Shubo, Christian all had a share in making to work here enjoyable and fun.

I would also like to thank all my friends who helped to think from time to time about other things than the brain and goal directed reaching.

At the end I'm very grateful for the emotional support and encouragement of my parents, my sister and the rest of my family. I could always count on them and several times they motivated me again, when things were not going that well. I'm glad you always keep faith with me. 



\section{Contents}

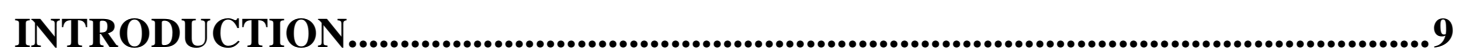

I. FRONTOPARIETAL REACH NETWORK ..............................................11

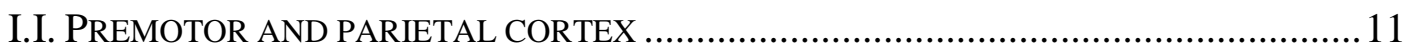

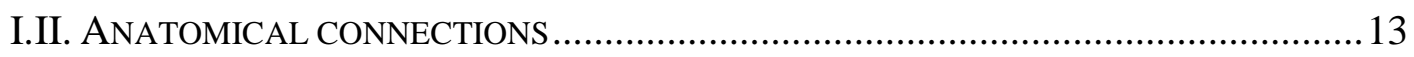

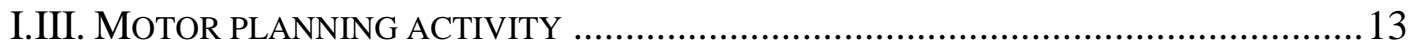

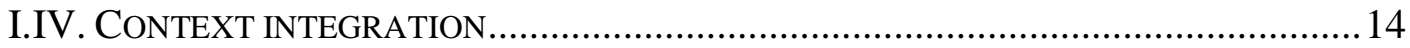

Implementation of Spatial Transformation Rules for Goal-Directed Reaching via

Gain Modulation in Monkey Parietal and Premotor Cortex.............................. 17

The Cortical Timeline for Deciding on Reach Motor Goals ............................... 36

Choosing Goals, Not Rules: Deciding among Rule-Based Action Plans ............ 55

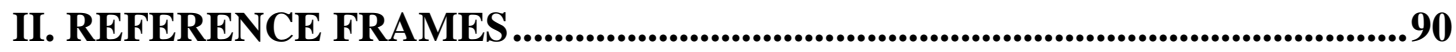

II.I. REACH PLANNING IN DIFFERENT REFERENCE FRAMES ..................................90

What is 'anti' about anti-reaches? - Reference frames selectively affect reaction times and endpoint-variability ...................................................................... 92

III. MOTOR LEARNING AND MOTOR ADAPTATION...................................102

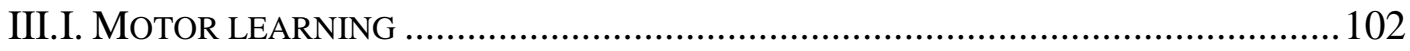

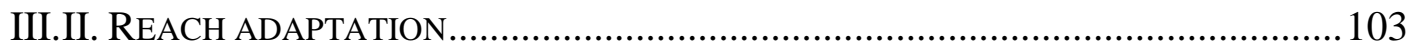

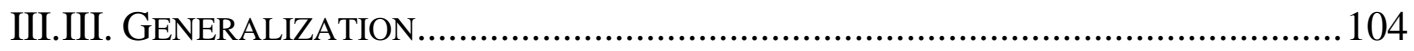

Asymmetric generalization in adaptation to target displacement errors .......... 105

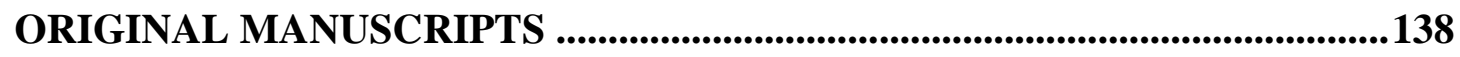

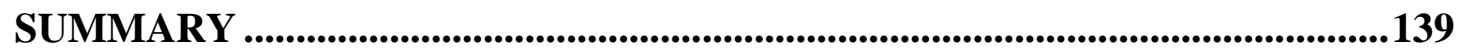





\section{Introduction}

Every day we use movements to interact with our environment. Even a relatively simple movement, such as reaching to a target requires the integration of multiple sensory signals from different modalities. Furthermore, with the same sensory input our actions and the goals of such actions are influenced by the context of the current situation (e.g. thirst or hunger) as well.

This thesis deals with the transformation of sensory information into goal directed reach movement plans in the brain and the influences of the contextual information on reach planning. It consists of five research articles dealing with the different aspects of the topic. Two different but complimentary approaches were used to investigate reach planning and how it is influenced by certain task rules or perturbations of the environment. First, I measured the spiking activity of single neurons in two brain areas of macaque rhesus monkeys, the dorsal premotor cortex (PMd) and the parietal reach region (PRR), both known to be involved in the planning of reach movements. The macaque rhesus monkey provides a good model organism as its reach capabilities are highly developed and similar to those of the human. Further, its cognitive abilities allow investigating influences of cognitive factors such as task rules, on reaching behavior and underlying brain function. Second, psychophysical methods were used to measure behavioral parameters of human reaching and motor learning behavior.

The thesis is organized in three chapters dealing with the cortical representations of reach plans, influence of reference frames on reach plans, and reach adaptation, respectively. At the beginning of each chapter a brief introduction provides a summary about the current literature and is followed by the original manuscripts.

The first chapter of the thesis deals with neuronal encoding of reach planning and consists of three original research articles and manuscripts. In the first manuscript, we investigate how spatial tuning properties of neurons in PMd and PRR are influenced by task rules and whether the neural encoding of the reach goal differs when the physical reaches are identical but the task rule is different. The second manuscript focuses on inter- and intra-areal response latencies and how these are modulated by 
task rules. The relative timing of reach goal encoding between PMd and PRR can be helpful in parsing out the direction of information flow within the frontoparietal reach network. The third manuscript explores situations in which the neuronal ensembles need to encode several equally likely possibilities instead of one pre-defined reach goal. Here, we characterize how the neurons in PMd and PRR respond to such multiple potential motor goals, and which of these available options are represented in these brain areas.

The second chapter deals with reference frames, which were investigated with psychophysical methods in humans and summarized in one manuscript. Unlike electrophysiology, psychophysical studies cannot yield information about neural activity in specific brain areas but can provide insights into possible neural mechanisms influencing behavioral parameters. The manuscript investigates how the relative position of a spatial instruction stimulus and the reach goal with respect to the eye and the hand influences behavioral parameters of reaching. Depending on whether the hand, eye, or a combination of both is used as a reference point, parameters such as reaction times and reach precision could be affected by the different neuronal processes during a reach.

The third chapter deals with reach adaptation as a form of motor learning. One manuscript analyzes reach adaptation and generalization in a human psychophysical study. Learning to correct for a disruption at one point in space transfers to other locations, and a study of the pattern of such transfers provides clues about how such kind of learning is implemented in the brain. 


\section{Frontoparietal reach network}

\section{I.I. Premotor and parietal cortex}

The ability for goal-directed reaching is highly developed in primates, including humans. Every day we execute hundreds of reach movements which are aimed at specific objects. Normally, these movements are guided by vision, but we are also able to reach to auditory or somatosensory defined targets. For those goal-directed movements multimodal sensory information has to be combined in order to compute the position of objects that serve as targets for goal-directed movements. In a classical feed-forward view visual information about the location of object is processed via the posterior parietal cortex as part of the dorsal processing pathway and transmitted to the frontal premotor cortex. The premotor cortex in turn, projects to the primary motor cortex (M1). While the parietal areas were thought to deal with multisensory integration (for review see Colby and Goldberg, 1999), combining multimodal sensory inputs to a visuo-spatial map, premotor and motor cortex were known to deal with the planning, initiation and directing of voluntary movements (Godschalk et al., 1981; Weinrich and Wise, 1982; Wise and Mauritz, 1985).

However, during the last years it has been shown that the parietal cortex itself is important for movement planning and decision making. The involvement of parietal cortex in movement planning was shown in electrophysiology studies in monkeys (e.g.: Mountcastle et al., 1975; Gnadt and Andersen, 1988), in human imaging studies (Grafton et al., 1992; Connolly et al., 2000; Connolly et al., 2003; Medendorp et al., 2005) as well as in lesion studies. Patients with a damage of the posterior parietal cortex (PPC), besides other possible deficits, were shown to suffer from deficits in localization of objects leading to problems in reaching (optic ataxia: Balint, 1909; Rondot, 1977), and from the inability to plan movements (apraxias: Geshwind \& Damasio, 1985) and to form the shape of the hand appropriately in preparation to grasp an object (Perenin and Vighetto, 1988; Goodale and Milner, 1992). These studies suggest that PPC is involved in integrating multisensory spatial information for the planning of goal directed movements. The PPC and the premotor cortex, which is located in the frontal cortex, form a highly interconnected network for movement planning (Pandya and Kuypers, 1969; Jones and Powell, 1970; Kurata, 1991; Johnson et al., 1996; Caminiti et al., 1999; Marconi et al., 2001; Tanne-Gariepy et al., 2002; Battaglia-Mayer et al., 2003). Within this network, various sub-areas are 
specialized for different effectors (Snyder et al., 1997; Connolly et al., 2000; Rushworth et al., 2001). For example, the lateral intraparietal area (LIP), which lies at the lateral part of the intraparietal sulcus, and the anatomical connected frontal eye fields (FEF) in the rostral bank of the arcuate sulcus are specialized for the planning of eye movements (Bruce and Goldberg, 1985; Bruce et al., 1985; MacAvoy et al., 1991; Mazzoni et al., 1996; Snyder et al., 1997; Snyder et al., 2000). Similarly, area AIP in the parietal and F5 in the ventral premotor cortex are specialized for hand (grasping) movements. Firing rate in neurons in these areas encode different grip types (e.g. power grip versus precision grip) as well as the wrist orientation of the hand (Rizzolatti et al., 1987; Rizzolatti et al., 1988; Sakata and Taira, 1994; Baumann et al., 2009) prior to the upcoming movement. Reach movement planning, in contrast, is encoded by the dorsal premotor cortex (PMd) and the functionally defined parietal reach region (PRR) (Weinrich et al., 1984; Wise et al., 1986; Kurata, 1989; Kurata, 1993; Snyder et al., 1997; Batista et al., 1999; Colby and Goldberg, 1999; Connolly et al., 2000; Rushworth et al., 2001; Calton et al., 2002). Anatomically, PRR overlaps mainly with the medial intraparietal area (MIP) and might include parts of area V6a and area 5. The following sections will focus on the properties of PMd and PRR since the electrophysiological recordings reported in the first part of this thesis have been conducted in these two areas. Figure 1 shows a schematic surface plot of a macaque monkey brain and MRI scans from one of the monkeys in which the experiments were done.
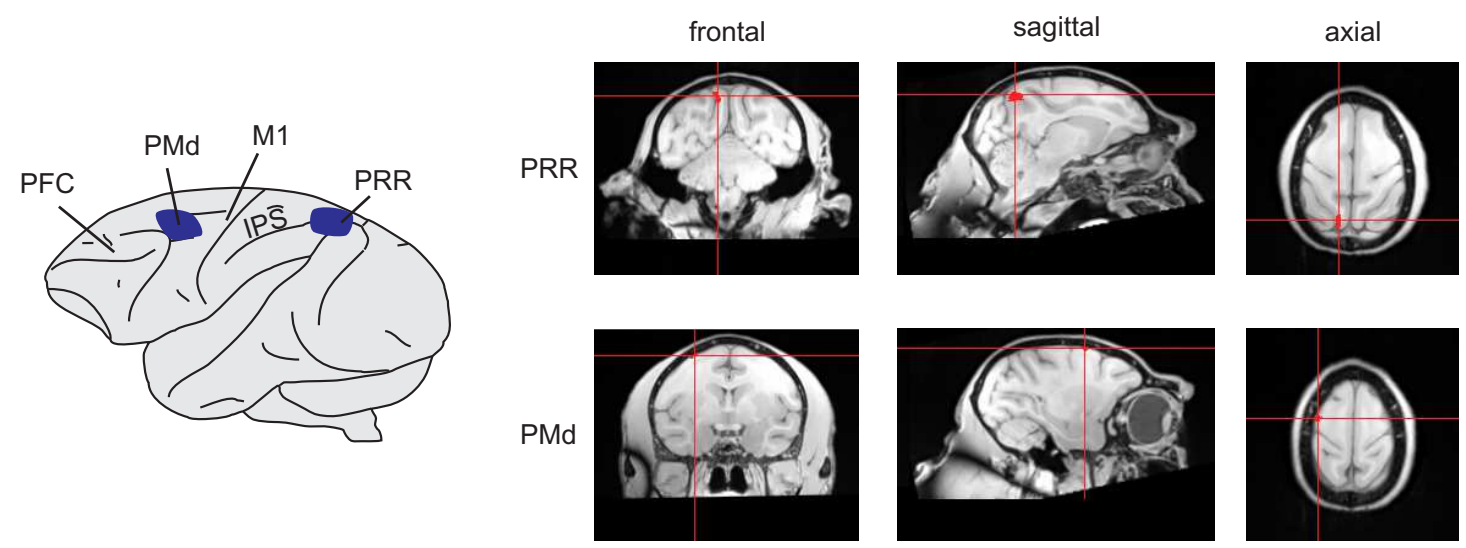

Figure 1: Right: Schematic surface plot a macaque monkey brain. The blue patches label the two brain areas, PMd and PRR, in which electrophysiological investigations were done. Note that for PRR the surface is labeled even though it lies within the intraparietal sulcus. Left: MRI scans from monkey A. Within the slices recording positions for PRR (top row) and PMd (bottom row) are marked. PFC: prefrontal cortex; PMd: dorsal premotor cortex; M1: primary motor cortex; IPS: intraparietal sulcus; PRR: parietal reach region 


\section{I.II. Anatomical connections}

The parietal occipital area (PO), which lies in the rostral bank of the parieto-occipital sulcus deep in the medial wall of the hemisphere, is the input structure for visual information into PRR (i.e. MIP). Area PO itself receives input from the primary visual cortex (Colby et al., 1988) and is reciprocally connected with PRR (Colby et al., 1988; Blatt et al., 1990). Anatomical studies of retrograde tracer injections in PMd show that it is robustly and reciprocally connected with PRR (Pandya and Kuypers, 1969; Jones and Powell, 1970; Kurata, 1991; Johnson et al., 1996; Caminiti et al., 1999; Petrides and Pandya, 1999; Marconi et al., 2001; Tanne-Gariepy et al., 2002; Battaglia-Mayer et al., 2003). PMd itself projects, beside other connections, to the primary motor cortex as well as direcly to the spinal cord (Rizzolatti and Luppino, 2001). Note that the described connections are not the complete projections of PRR and PMd, but just meant as an overview.

\section{I.III. Motor planning activity}

Neurons in PMd and PRR are thought to be involved in reach movement planning. Electrophysiology studies in monkeys have shown that in both areas neurons are spatially selective for the direction of an upcoming reach. The firing rate of a single neuron is modulated by the direction of the upcoming reach, with reaches in the preferred direction of the neuron leading to increases in its firing rate. The response of the same neuron is diminished if the monkey is preparing a reach in the opposite direction whereas it responds at some intermediate level if the upcoming reach is in orthogonal directions. The activity in PMd and PRR thought to relate to motor planning rather than execution, because the neurons can become active up to several seconds before the actual movement starts. In so-called delay-reach tasks the neuron gets active - with some latency - as soon as the monkey knows the reach goal even if it has to withhold its movements up to several seconds (e.g.: Gnadt and Andersen, 1988; Snyder et al., 1997; Batista and Andersen, 2001). However, those tasks do not allow differentiating whether the activity of the neurons does really reflect movement planning or rather reflects the visual memory encoding the spatial location of the instruction stimulus. The dissociation between visual memory and motor goal tuning is possible with an anti-reach task (Crammond and Kalaska, 1994; Gail and Andersen, 
2006). In these tasks the monkey sees a peripheral spatial instruction stimulus and, depending on the current valid task rule, it has to reach, starting from a central position, either toward this spatial cue or opposite to it. If a neuron's response varies with the position spatial instruction stimulus regardless of whether it has to reach toward or opposite to it, it would indicate that the neuron reflects visual memory. If, in contrast, a neuron's response varies with the movement direction, irrespective of whether the direction is defined by direct reach or anti-reach instruction that would indicate that the response of the neuron is related to the direction of the reach and the neuron is therefore tuned for the motor goal.

\section{I.IV. Context integration}

In order to define a reach goal it is not sufficient to gather all spatial information about the objects in our environment. In our daily life, we often encounter several objects of which we interact with only selected ones. Internal states can influence our decision about a reach goal. At a table, we choose a glass of water when we are thirty but a sandwich when we are hungry. In a different situation, abstract learned rules might decide our motor behavior. We know the visuomotor association of stopping at a red traffic light, and walking at a green one. The information about the context, which might be an internal state or a learned visuomotor association, has to be integrated with the spatial information about objects in the environment to define the final reach goal/motor plan. How and where does this integration happen?

It is known that neurons in the frontal areas of the cortex are selective for task rules (Boussaoud and Wise, 1993; Asaad et al., 1998; Wallis et al., 2001; Wallis and Miller, 2003). This was e.g. tested in a task (match-to-sample task) where a monkey was presented sequentially with images of two objects and depending on the current task rule had to release a lever either if the two objects were the same (same-rule) or if they were different (different-rule). Neurons in the prefrontal cortex (PFC), rostral to the arcuate sulcus, were selective for these task rules. For example, they responded whenever the same-rule was valid but not for the different-rule, or vice versa (Hoshi et al., 1998; Wallis et al., 2001; Johnston and Everling, 2006). Similarly, PFC neurons were shown to represent arbitrary visuomotor associations in different tasks (Asaad et al., 1998; White and Wise, 1999; Everling and Desouza, 2005) Furthermore, human 
imaging data also show activation of PFC during learning of arbitrary cue-response associations (Boettiger and D'Esposito, 2005; Hanakawa et al., 2006).

Within the fronto-parietal reach network, neurons even though mainly selective for the direction of an upcoming movement, are also modulated by a certain context or a task rule. Neurons in the premotor cortex e.g. respond differently if different cues instruct the same movement or, if different rules instruct the same behavioral response (Boussaoud and Wise, 1993) and they also show selectivity to task rules in the above mentioned match-to-sample task (Wallis and Miller, 2003). More recently, it has been shown that a population of neurons in the PPC also encodes the currently valid task rule (Stoet and Snyder, 2004; Gail and Andersen, 2006; Gail et al., 2009). In the above mentioned anti-reach tasks, for both PMd and PRR (Crammond and Kalaska, 1994; Gail and Andersen, 2006) it has been shown that during a delay, in which the monkey already knows the reach goal but is not allowed to execute the movement, neurons indeed encode the upcoming motor goal and not just the position of the spatial cue. This is true for nearly all spatially tuned neurons and shows that at this processing level the spatial information is already integrated with the contextual information. However, some of those neurons, if analyzed during an earlier time window after the onset of the cue, represent the spatial position of the cue instead of the motor goal. Those neurons therefore show a dynamic visuomotor tuning, i.e. they are first visually tuned but after a while, when the context information is integrated, the same neuron switches its representation and the tuning now encodes the motor goal. These examples show evidence that within the fronto-parietal reach network the integration of spatial information with the contextual information is achieved. But where within this network does the integration of the context information with the spatial information about object happen, such that context dependent goal-directed reaching is possible?

Within the fronto-parietal reach network the anatomical position of the premotor cortex makes it an ideal candidate as an interface for integrating the spatial sensory information about reach goal positions with contextual information. As mentioned above PMd receives projections from the dorsal pathway via PRR (Pandya and Kuypers, 1969; Jones and Powell, 1970; Kurata, 1991; Johnson et al., 1996; Caminiti et al., 1999; Marconi et al., 2001; Tanne-Gariepy et al., 2002; Battaglia-Mayer et al., 2003) and from the frontal cortex (Barbas and Pandya, 1987; Lu et al., 1994; Wise et al., 1997). In the classical feed forward view, PMd would combine the spatial 
information received via the parietal cortex with the context information received via the prefrontal cortex into a motor goal. This information of the motor goal would then be sent to M1, which is the output stage to the spinal cord. If this strict feed-forward view is true, why then do we see contextual tuning and modulation also in PRR, which has no direct connection to M1? One interpretation for parietal motor goal tuning comes from a theoretical approach (Brozovic et al., 2007), which suggested that a feedback projection from motor output structures to sensory motor layers instead of classical feed-forward projections mediates the visuomotor transformation. The integration of the context within this network is achieved by gain-field mechanisms. In such a mechanism the directional tuning of a neuron would be constant for different contexts (i.e. a given neuron would always respond best if the upcoming reach is to the right), but the overall strength of the response would change with the context (i.e. the same neuron would be more active if the upcoming (e.g. rightward) reach is directed towards a spatial cue than if the reach is directed opposite). In the first research article of this thesis, we tested this prediction of the model for the visuomotor transformations in the fronto-parietal reach network. A further prediction from the model is that the motor goal should be represented earlier in PMd than in PRR. The second research article of this PhD-thesis tested this prediction. Furthermore it investigates the influence of spatial remapping rules as well as motor commands on motor goal latencies within PMd and PRR. 


\title{
Implementation of Spatial Transformation Rules for Goal-Directed Reaching via Gain Modulation in Monkey Parietal and Premotor Cortex
}

\author{
Alexander Gail, Christian Klaes, and Stephanie Westendorff \\ Bernstein Center for Computational Neuroscience, German Primate Center-Leibniz Institute for Primate Research, 37077 Göttingen, Germany
}

\begin{abstract}
Planning goal-directed movements requires the combination of visuospatial with abstract contextual information. Our sensory environment constrains possible movements to a certain extent. However, contextual information guides proper choice of action in a given situation and allows flexible mapping of sensory instruction cues onto different motor actions. We used anti-reach tasks to test the hypothesis that spatial motor-goal representations in cortical sensorimotor areas are gain modulated by the behavioral context to achieve flexible remapping of spatial cue information onto arbitrary motor goals. We found that gain modulation of neuronal reach goal representations is commonly induced by the behavioral context in individual neurons of both, the parietal reach region (PRR) and the dorsal premotor cortex (PMd). In addition, PRR showed stronger directional selectivity during the planning of a reach toward a directly cued goal (pro-reach) compared with an inferred target (anti-reach). PMd, however, showed stronger overall activity during reaches toward inferred targets compared with directly cued targets. Based on our experimental evidence, we suggest that gain modulation is the computational mechanism underlying the integration of spatial and contextual information for flexible, rule-driven stimulus-response mapping, and thereby forms an important basis of goal-directed behavior. Complementary contextual effects in PRR versus PMd are consistent with the idea that posterior parietal cortex preferentially represents sensory-driven, "automatic" motor goals, whereas frontal sensorimotor areas are stronger engaged in the representation of rule-based, "inferred" motor goals.
\end{abstract}

\section{Introduction}

The same visual scene can lead to very different actions taken depending on the behavioral context. In a real boxing match, the face of your opponent is the direct goal of your arm movement. In a show fight, the face is not the goal of the movement but still guides it. Planning a reach toward a visual target object or inferring a reach goal from the position of an object according to a spatial transformation rule requires context-specific sensorimotor transformations (Gail and Andersen, 2006). Here, we test how the frontoparietal reach network flexibly creates context-specific motor-goal representations.

Spatially flexible cue-response mapping, as in a pro-reach/ anti-reach task, requires integration of spatial sensory information with the context. Computational models suggest that this flexibility can be achieved with contextual gain modulation of spatially selective neurons (Salinas, 2004; Brozović et al., 2007), equivalent to gain field mechanisms suggested for multisensory integration (Andersen et al., 1985; Zipser and Andersen, 1988; Boussaoud et al., 1993, 1998; Brotchie et al., 1995; Galletti et al.,

Received March 5, 2009; revised June 13, 2009; accepted June 16, 2009.

This work was supported by the Federal Ministry for Education and Research (Germany) Grants 01600433 and 01GQ0814. We thank Sina Plümer and Ludwig Ehrenreich for their extensive support in data collection, and Axel Lindner for helpful discussions on previous versions of this manuscript.

Correspondence should be addressed to Dr. Alexander Gail, Bernstein Center for Computational Neuroscience, German Primate Center-Leibniz Institute for Primate Research, Kellnerweg 4, 37077 Göttingen, Germany. E-mail: agail@gwdg.de.

D0I:10.1523/JNEUROSCI.1095-09.2009

Copyright $\odot 2009$ Society for Neuroscience $\quad$ 0270-6474/09/299490-10\$15.00/0
1995; Salinas and Abbott, 1996; Snyder et al., 1998; Salinas and Thier, 2000). If the idea of gain modulation for space-context integration holds true, then spatially selective neurons in primate cortical sensorimotor areas, like the parietal reach region (PRR) and the dorsal premotor cortex (PMd), should be upregulated and downregulated by the behavioral context.

Spatial sensory information presumably reaches the frontoparietal sensorimotor network via the posterior parietal cortex (Colby et al., 1988; Blatt et al., 1990; Marconi et al., 2001). Associative goal selection criteria or arbitrary transformation rules are believed to exert their influence on motor planning via prefrontal and premotor areas (Rushworth et al., 1997; Wise and Murray, 2000; Toni et al., 2001; Wallis and Miller, 2003). Both PMd and PRR have been shown to express sustained spatially selective activity during movement planning (Weinrich and Wise, 1982; Andersen et al., 1985; Boussaoud and Wise, 1993; Kalaska, 1996; Wise et al., 1997; Andersen and Buneo, 2002; Cisek, 2007), predominantly representing motor goals (di Pellegrino and Wise, 1993; Crammond and Kalaska, 1994; Gail and Andersen, 2006). We compare contextual modulations in PRR and PMd to test whether the two areas represent inferred versus directly cued motor goals differently. Posterior parietal cortex, for example, was hypothesized to mainly represent fast "automatic" motor goals (Desmurget et al., 1999; Pisella et al., 2000), as in pro-reaches.

We tested whether and how motor-goal representations in PRR and PMd are modulated by context-specific spatial transformation rules during reach planning. We used a memory-guided 
a

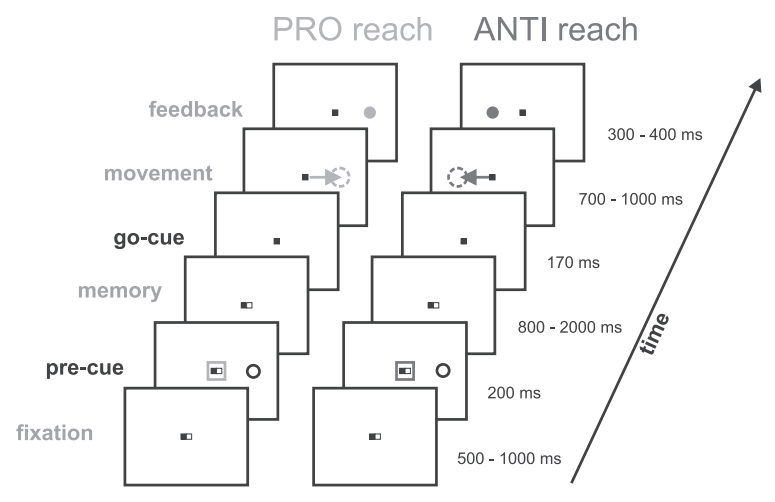

b

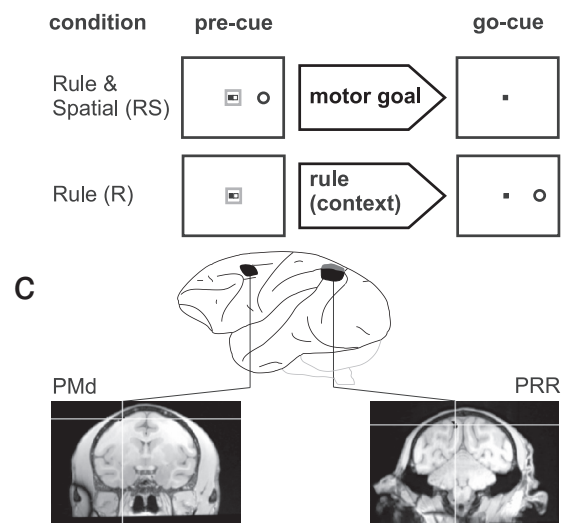

Figure 1. Memory-guided anti-reach task with precuing. $\boldsymbol{a}$, The left sequence shows an example of a pro-trial, and the right, an example of an anti-trial. Subjects had to maintain eye fixation (central dark spot) throughout the trial and hand fixation (central white spot) until the go instruction (disappearance of the white spot). The reach goal was defined by the combination of a context cue (colored central frame), indicating the pro/anti transformation rule, and a color-neutral spatial cue, which was presented at any of four different peripheral screen positions $\left(0,90,180,270^{\circ}\right)$. Cues could be presented before (precue period) or after a variable memory period (go cue period), simultaneously or separately. In the example, the context and the spatial cue are both presented during the precue period. To be rewarded, the subject had to make a reach toward the previous spatial cue position (pro-reach) or to the opposite side (anti-reach). The arrows and dotted circles are for demonstration purpose only and were not shown to the subjects. $\boldsymbol{b}$, In this study, we compare conditions in which the subjects either had full information on the upcoming motor goal (transformation rule and spatial cue presented as precue; RS condition) or only the context information available (transformation rule presented as precue, spatial cue during go cue period; R condition). c, Extracellular signals from multiple individual neurons were recorded simultaneously in PRR and PMd (regions of interest for monkey S) while the monkeys performed the task.

pro-reach/anti-reach task with partial precuing to investigate contextual modulations that were either independent or dependent of the spatial tuning. We tested separately the effect of context on either the response amplitude or the directional selectivity. Our results are consistent with the idea of space-context integration by a gain modulation mechanism. Additionally, we found complementary effects of context on motor-goal representations in PRR and PMd, which indicate different mutual roles of the two areas in context-specific visuomotor integration and motor-goal representation.

\section{Materials and Methods}

Tasks and control of behavioral parameters. Two male rhesus monkeys (Macaca mulatta; S and A) were trained to perform a memory-guided anti-reach task with partial precuing (Fig. 1a). Two visual cues, one spatial and one contextual, were presented to the subjects either simultaneously or at different points in time (19 inch ViewSonic LCD VX922; $5 \mathrm{~ms}$ off-on-off response time). The contextual cue (transformation rule) consisted of a green (pro-reach) or blue (anti-reach) frame around the eye and hand fixation points and instructed the subject to reach either toward (pro) or diametrically opposite to the spatial cue (anti). The peripheral spatial cue was located at one of four possible positions $(0,90$, 180 , and $270^{\circ}$ ) with an eccentricity of $9 \mathrm{~cm}\left[14.5^{\circ}\right.$ visual angle (VA)] relative to the fixation point. The motor goal was only defined uniquely, once both cues were known to the subject.

The monkey initiated a trial, by fixating a small red square in the center of the screen (eye fixation tolerance, 2.5-4. $0^{\circ} \mathrm{VA} ; 224 \mathrm{~Hz} \mathrm{CCD}$ camera; ET-49B; Thomas Recording) and touching an adjacent white square of the same size (hand fixation tolerance: $4.0^{\circ} \mathrm{VA}$, touch screen mounted directly in front of the video screen; IntelliTouch; ELO Systems). After a short period of 500-1000 ms (fixation period), the precue appeared for $200 \mathrm{~ms}$. The precue could be either the contextual and the spatial cue at once [rule and spatial condition (RS)], only the spatial cue [spatial-only condition (S)], only the contextual cue [rule-only condition (R)], or nothing [null condition $(\mathrm{N})$ ], leading to four different memory conditions. After the precue, the monkey had to keep eye and hand fixation for 800-2000 ms (memory period). Depending on the memory condition, additional information was given to the monkey at the end of memory period. In the $\mathrm{R}, \mathrm{S}$, and $\mathrm{N}$ conditions, the missing information required to fully specify the reach goal (the spatial cue, the contextual cue, or both) was briefly flashed during the $170 \mathrm{~ms}$ go cue period. In the RS condition, no additional information was shown. Simultaneously, with the presentation of the second cue, the hand fixation square disappeared ("go" signal) and the monkey had to reach toward the instructed goal within a maximum of $700-1000 \mathrm{~ms}$ (movement period; $4.9^{\circ} \mathrm{VA}$ reach tolerance) and hold the goal position for $300-400 \mathrm{~ms}$ (feedback period). The monkey received visual feedback about the correct movement goal, which consisted of a filled circle of the same color as the contextual cue and was presented at the goal location during the feedback period. Eye fixation had to be kept throughout the trial; otherwise, the trial was aborted immediately. Liquid reward and acoustic feedback indicated correct (high pitch tone, reward) or incorrect (low pitch tone, no reward) behavior.

Pro-trials and anti-trials were randomly interleaved from trial to trial ( $\sim 400$ trials per data set) and the four memory conditions were randomly mixed in small blocks (10-20 trials of same condition per block). To test the contextual influence on motor planning, only the results of the RS and R conditions (transformation rule available during the planning phase of the movement) are meaningful and presented here (Fig. 1b).

Animal preparation and neural recordings. After training of the task, both monkeys were implanted with titanium head holders that were custom-fit to their heads based on computer-tomographical surface reconstruction of the skull (3di $\mathrm{GmbH})$. After recovery and additional training, each monkey was implanted with two magnetic resonance imaging (MRI)-compatible recording chambers, also custom-fit to the skull. One chamber was placed above PRR [Horsley Clarke coordinates: $6 \mathrm{~mm} /-8.5 \mathrm{~mm}$ (monkey S/A) lateral; $-10 \mathrm{~mm} /-9.5 \mathrm{~mm}$ anterior], the other chamber above PMd (13 mm/- $13.5 \mathrm{~mm}$ lateral; $17 \mathrm{~mm} / 19.8 \mathrm{~mm}$ anterior). Presurgical structural MRI was used to position the chambers (Fig. 1c). Postsurgical MRIs, showing the chamber relative to the brain, verified the correct chamber positions and allowed precise targeting of the desired anatomical structure. Sustained, direction-selective, neural responses during center-out reach planning was used as a physiological signature in both areas to confirm the imaging-based positioning. Both chambers were implanted contralaterally to the handedness of the monkey (A, left hemisphere; S, right hemisphere). All surgical and imaging procedures were conducted under general anesthesia.

For the extracellular recordings, up to four microelectrodes in each cortical area in a five-channel microdrive ("mini-matrix"; Thomas Recording) were used simultaneously. In most sessions, simultaneous recordings were conducted in both areas. The raw signals were preamplified (20×; Thomas Recording), bandpass filtered, and amplified (154 
$\mathrm{Hz}$ to $8.8 \mathrm{kHz}$; $400-800 \times$; Plexon), before on-line spike-sorting was conducted (Sort Client; Plexon). In addition to spike times, the spike waveforms were recorded and later subjected to off-line sorting for optimal isolation quality (Offline Sorter; Plexon).

Animal care and all experimental procedures were conducted in accordance with German law (Animal Welfare Act).

Selection of neural data. All recorded and sufficiently well isolated units, regardless of their tuning properties, were included in the neural data analyses unless explicitly stated otherwise. We analyzed the neural activity during the last $300 \mathrm{~ms}$ of the memory period (i.e., activity succeeding the precue with a time lag of at least $500 \mathrm{~ms}$, and immediately preceding the go cue). This period was chosen to extract movement planning activity without confounding effects of (1) immediate visual input from the cue stimuli, or (2) transition phases from visual to motor-goal tuning (Gail and Andersen, 2006), or (3) visual and somatosensory input and motor-control signals related to movement initiation.

Analyses of neuronal directional selectivity. Directional selectivity was quantified with a directional tuning vector (DTV), which is defined as the vector average across all center-out cue directions $\vec{u}_{i}$ (unit vectors) weighted with the corresponding mean neural spike rates across trials with this cue direction $\left(r_{i j}\right)$ and normalized to the total mean spike rate across all trials for the neuron $j$ as follows:

$$
\operatorname{DTV}_{j}=\sum_{i=1}^{4} r_{i j} \vec{u}_{i} / \sum_{i=1}^{4} r_{i j}
$$

The length of the resulting vector is between 0 and 1 and is a measure of the tuning strength. Its direction will be referred to as preferred direction (PD) of a neuron. The DTV was computed separately for pro-trials and anti-trials and was defined relative to the position of the spatial cue, not relative to the movement goal.

Significance of the directional selectivity was tested with a nonparametric one-way ANOVA (Kruskal-Wallis; Matlab; Mathworks) with the four different visual cue directions as groups and sample sizes defined by the number of identical trial repetitions. The ANOVA was calculated independently within each transformation rule (pro/anti) and precuing condition (total of $2 \times 4=8$ combinations).

The relative difference in PD between pro-trials and anti-trials in the RS condition indicates whether the tuning of a neuron in the given time window reflects the visual cue or the reach goal position. We quantified this difference with the tuning direction difference index (DD) as follows:

$$
\mathrm{DD}=\mathrm{PD}_{A}-\mathrm{PD}_{P}
$$

$\mathrm{PD}_{P}$ and $\mathrm{PD}_{A}$ are the preferred directions of a neuron in pro-trials and anti-trials, respectively. A DD of $\pm 0^{\circ}$ indicates idealized visual tuning. A $\mathrm{DD}$ of $\pm 180^{\circ}$ means opposing PDs (relative to the cue position), which indicates idealized motor-goal tuning.

Analyses of contextual modulations. We tested three different effects of the context on the neuronal activity and quantified each of them with a contextual modulation index: gain modulation of spatially selective neurons (i.e., amplitude changes independent of changes in directional selectivity [gain modulation (GM)], changes in directional selectivity independent of changes in overall neural response strength [selectivity modulation $(\mathrm{SM})]$, and direct modulation of neuronal activity levels independent of spatial tuning [direct modulation (DM)]).

Contextual gain modulation is characterized by amplitude changes of spatial tuning, independent of changes in directional selectivity. It is defined as follows:

$$
\mathrm{GM}=\frac{r_{P}^{\mathrm{RS}}-r_{A}^{\mathrm{RS}}}{r_{P}^{\mathrm{RS}}+r_{A}^{\mathrm{RS}}}
$$

where $r_{P}^{\mathrm{RS}}$ and $r_{A}^{\mathrm{RS}}$ are the mean spike rates for pro-trials and anti-trials in the RS condition. Since most neurons in PRR and PMd are spatially highly selective with a DD of around $\pm 180^{\circ}$ (see Results), large absolute GM values indicate strong gain modulation effects of the spatial motorgoal tuning by the context.
Contextual selectivity modulation is characterized by changes in directional selectivity independent of changes in response amplitude. To quantify contextual selectivity modulation, we computed the contrast of the normalized DTV length between pro-trials and anti-trials in the RS condition as follows:

$$
\mathrm{SM}=\frac{\left|\mathrm{DTV}_{P}\right|-\left|\mathrm{DTV}_{A}\right|}{\left|\mathrm{DTV}_{P}\right|+\left|\mathrm{DTV}_{A}\right|},
$$

where $\mathrm{DTV}_{P}$ and $\mathrm{DTV}_{A}$ are the normalized directional tuning vectors for pro-trials and anti-trials. A positive SM indicates that a neuron is more strongly tuned in pro-trials than in anti-trials, and vice versa for negative values. Values close to zero indicate that tuning selectivity is not modulated by the transformation rule.

Both GM and SM quantify modulations of spatial tuning by context. GM and SM are independent of each other in that each can change without affecting the other by varying parameters of hypothetical tuning functions (supplemental material S1, available at www.jneurosci.org). This does not mean that a single cell could not simultaneously have a high GM and SM. In fact, for neurons that are very strongly modulated by the context the activity in one of the two conditions (pro or anti) may be so weak that the tuning becomes arbitrarily shaped, which can lead to large GM and SM values and "random" DD values.

Direct context modulation can be quantified by differences in the memory activity between pro-reaches and anti-reaches in the R condition, since these differences reflect contextual influence independent of any spatial tuning. We defined the direct context modulation as the contrast of the average activities between pro-trials and anti-trials as follows:

$$
\mathrm{DM}=\frac{r_{P}^{R}-r_{A}^{R}}{r_{P}^{R}+r_{A}^{R}} .
$$

$r_{P}^{R}$ and $r_{A}^{R}$ are the mean spike rates in the $\mathrm{R}$ condition for pro-trials and anti-trials, respectively. A positive DM indicates neurons that are more active in pro-trials. A negative DM indicates stronger activity in anti-trials. Values close to zero indicate neurons indifferent to the transformation rule.

Note that all modulation indices are nonparametric and do not require fitting of any predefined tuning functions.

All contextual modulations were statistically analyzed on the level of individual neurons and, additionally, on the population level. We used bootstrap methods ( $n=100$ samples) to estimate the confidence limits of all indices (GM, SM, DM, and DD) for each individual neuron. The trial-by-trial spike rates were randomly permuted with repetitions, whereas the assignment of each trial to a certain direction, transformation rule, and memory condition was kept unchanged. GM, SM, and DM were considered significant if the $95 \%$ confidence limit did not overlap with zero. DD was considered to significantly deviate from motor-goal tuning if the circular confidence interval did not overlap with $180^{\circ}$.

On the population level, we estimated the to-be-expected distributions of all indices when assuming random data (shuffle test; $n=100^{*}$ number of used neurons). We randomly permuted trial-by-trial spike rates across the two transformation rules, while keeping the cue direction and memory condition unchanged. This procedure eliminated any effect of the transformation rule. For the GM, SM, and DM, $t$ tests were used to quantify deviations of the population means from zero. Additionally, a Bartlett test was used to test for deviations of the original distribution's variance from the normally distributed shuffled data. A positive Bartlett test indicates contextual modulations beyond what is expected because of random variations, even if the distribution of indices is centered at zero (i.e., even when there is no bias of the index toward either propreference or antipreference). To test whether a distribution of DD across the population of neurons deviates significantly from the predefined $\pm 180^{\circ}$ direction, we used circular statistics to compute the $95 \%$ confidence limits according to the following:

$$
d=\arccos \left[\frac{\sqrt{\frac{2 n\left(2 R^{2}-n \chi_{\alpha, 1}^{2}\right)}{4 n-\chi_{\alpha, 1}^{2}}}}{R}\right],
$$

where $R=n r . n$ is the number of samples, $r$ is the mean resultant vector length of the angular direction data (here, DD indices), and $\chi$ is the 
a

Classical motor goal cell $\mathrm{SM}=0.07, \mathrm{GM}=-0.02(P M d$, monkey $S)$

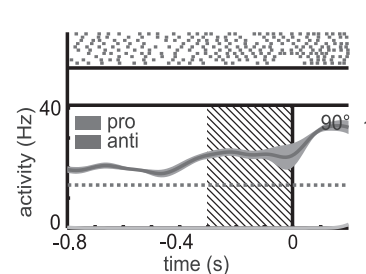

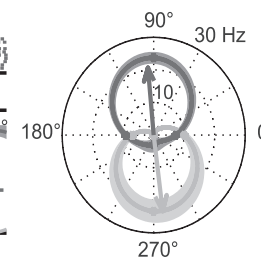

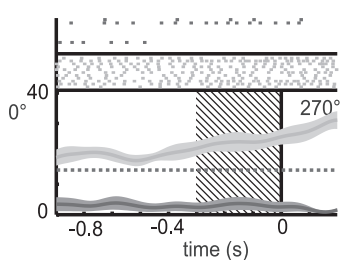

b

Relative tuning direction difference (DD)

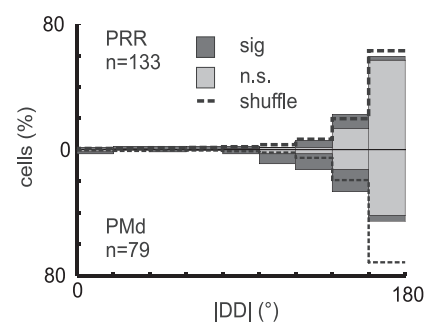

Figure 2. Motor-goal tuning in PRR and PMd during the late planning phase of reach movements. The example cell (a) shows classical motor-goal tuning, characterized by a PD depending on the motor goal and not the cue (DD not significantly different from $180^{\circ}$ ), as shown in the polar plot (middle). The response strength and selectivity were independent of the behavioral context (GM and SM not different from zero). Raster plots and spike density functions for pro (light gray) and anti (dark) trials are shown for the two most active directions (here, 90 and $270^{\circ}$ ) during the late memory period. Time 0 marks the onset of the go cue period. The mean firing rate during the fixation period is provided for comparison (dashed line). Spike density curves are smoothed with a Gaussian kernel ( $\sigma=50 \mathrm{~ms}$; dark lines, mean; light area, SE) for presentation purposes only. Also, polar tuning functions are interpolated for presentation purposes only, whereas all analyses are conducted nonparametrically on the original spike data. $\boldsymbol{b}$, Tuning DDs in PRR (top) and PMd (bottom) indicate that neurons in these areas are preferentially motor-goal tuned. Most individual neurons do not deviate significantly from $180^{\circ}$ (light gray bars); a few do according to their bootstrap confidence limits (dark bars). The circular distributions of the DD for all neurons in each area do not significantly deviate from $180^{\circ}$. Note that because of symmetry the distribution of absolute values (|DD|) are plotted, whereas circular statistics are applied to the original DD values.

inverse of the $\chi^{2}$ cumulative distribution function with a confidence limit of $1-\alpha$ (Zar, 1999).

To quantify the correlation between the signs and strengths of different forms of contextual modulation in individual neurons, we computed Pearson's cross-correlation coefficients of the different indices across the population of neurons. To make the modulation indices scale-invariant, we normalized the modulation indices to the variances of their respective distributions as follows:

$$
z_{i}=\frac{x_{i}}{\sigma_{i}}
$$

Here, $i$ is the considered index (GM, SM, DM), $x$ is the value of the index, and $\sigma$ is the variance of the index distribution over the cell population. Note that this normalization is only for easier comparison of the modulation strengths in the scatter plots of Figure 7. The cross-correlation coefficient is independent of this linear scaling, which is applied within each index distribution. To test whether different types of modulation tend to occur in complementary neuronal subpopulations, regardless of the "direction" of modulation (i.e., propreference vs antipreference), we also compared the absolute values of the different modulation indices. We used Spearman's rank correlation to account for the non-normal distribution of absolute modulation indices.

\section{Results}

Both monkeys performed the task with high accuracy. The percentage of overall correct trials was 78\% (monkey S) and $86 \%$ (monkey A) in the RS condition, and 75\% (S) and $83 \%$ (A) in the $\mathrm{R}$ condition. Error trials were mainly attributable to early trial abortion (mostly breaks in ocular fixation) but not attributable to a confusion of the "pro" and "anti" rules. The percentage of correct pro-reach and anti-reach decisions in otherwise-correct trials was 98\% (S) and 99\% (A) in the RS condition, and 94\% (S) and $99 \%$ (A) in the R condition. The performance of both monkeys showed no significant difference between pro-trials and anti-trials in the RS condition ( $p>0.05$, paired $t$ test), and a minimal difference in the $\mathrm{R}$ condition ( $\mathrm{S}: m_{\text {pro }}=95 \%, m_{\text {anti }}=94 \%, p=$ 0.008; A: $m_{\text {pro }}=99 \%, m_{\text {anti }}=98 \%, p=0.003$, paired $t$ test).

A total of 258 neurons from PRR (monkey S, 99; A, 159) and 193 from PMd (S, 75; A, 118) were recorded. Regardless of their spatial tuning properties, we tested all recorded neurons for direct context modulation (i.e., modulations of neuronal response amplitude by the context independent of directional selectivity) during the late memory period in the R condition (DM) (see Materials and Methods). For analyzing the effect of context on directional tuning properties (GM and SM) (see Materials and Methods and supplemental material S1, available at www. jneurosci.org, for details on modulation indices), we used a subset of neurons that had significant directional selectivity in at least one of the two spatial transformation conditions (pro/anti) during the late memory period of the RS condition. A total of 205 (79\%) neurons in PRR [S, 71 (71\%); A, $134(84 \%)]$ and 132 (68\%) neurons in PMd [S, 56 (75\%); A, 76 (64\%)] fulfilled this criterion. Data from both monkeys showed the same results and will be presented jointly (for comparison of the two monkeys, see supplemental material S2, available at www.jneurosci.org).

\section{Contextual gain modulations of motor-goal tuning}

When the reach goal was known to the monkeys during the memory period (RS condition), most of the spatially selective neurons in PRR $(82.0 \%)$ and PMd (72.2\%) were tuned for the motor goal, as was expected from previous results (Crammond and Kalaska, 1994; Gail and Andersen, 2006). Some individual neurons in the current study showed "classical" motor-goal tuning, which was independent of the spatial transformation rule that led to this motor goal (i.e., independent of the context). In the example neuron (Fig. $2 a$ ), the preferred directions for procondition and anticondition were opposite to each other when measured relative to the position of the cue, which means they were aligned with the motor goal (tuning direction difference DD not different from $180^{\circ} ; p>0.05$; bootstrap test) (see Materials and Methods), and their response strength as well as the directional selectivity did not differ between the pro and the anti condition (GM and SM not different from $0 ; p>0.05$; bootstrap test) (see Materials and Methods and below). The population of neurons in both areas on average represented the direction of the motor goal and not the cue position. In other words, the distribution of DD across the population of neurons did not deviate from $180^{\circ}$ neither in PRR (circular mean, $179 \pm 8^{\circ} \mathrm{SEM}$ ) (see Materials and Methods) nor in PMd (mean, $184 \pm 10^{\circ}$ ) (Fig. $2 b$ ). To be included in this analysis, the cells had to be significantly tuned in both spatial transformation conditions (pro/anti). This criterion was met by 133 (52\%) of all neurons in PRR [S, 50 (51\%); A, 83 $(52 \%)]$ and 79 (41\%) of all neurons in PMd [S, 35 (47\%); A, 44 $(37 \%)]$.

To test for contextual effects on spatial tuning properties, we distinguished between contextual GM and contextual SM (see 
a pro-preferring motor goal cell $\mathrm{SM}=0.09, \mathrm{GM}=0.36(P M d$, monkey $A)$
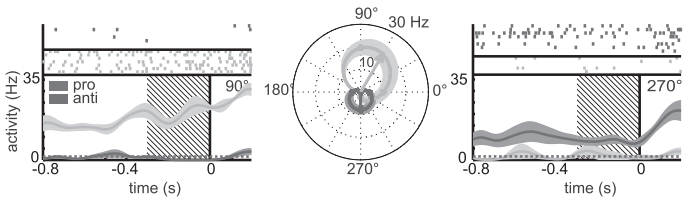

\section{anti-preferring motor goal cell} $\mathrm{SM}=0.05, \mathrm{GM}=-0.38(P R R$, monkey $A)$

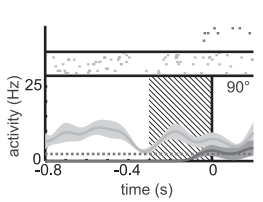

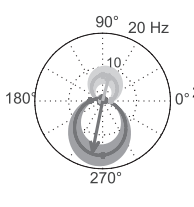

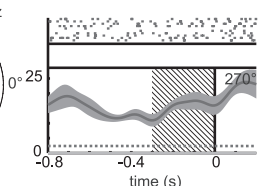

b

Gain modulation (GM)

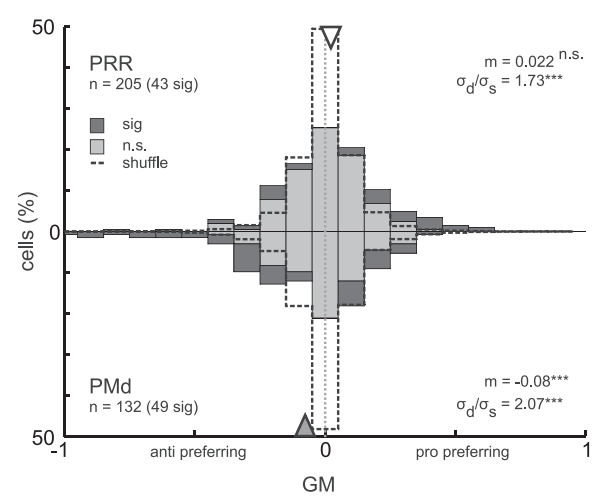

Figure 3. Contextual gain modulation of motor-goal tuning in PRR and PMd. $\boldsymbol{a}$, Examples of neurons with a high absolute GM indicating stronger activity for either pro-reaches (top) or anti-reaches (bottom), with invariant motor-goal tuning preference (DD not significantly different from $180^{\circ}$ ) and selectivity ( $\mathrm{SM}$ not significantly different from zero; conventions are as in Fig. $2 a$ ). The anti-preferring neuron (bottom) was also one that fulfilled the strict criteria for an ideal contextual gain modulation of motor-goal tuning: the neuron was significantly tuned in pro and anti trials, had a significant $\mathrm{GM}$, the $\mathrm{DD}$ was not significantly different from $180^{\circ}$, and the $\mathrm{SM}$ not significantly different from zero. (Note that the tuning vectors shown in the polar plot are not normalized, resulting in unequal length for pro and anti trials despite equally strong tuning; the SM is computed with normalized tuning vectors.) $\boldsymbol{b}$, Distribution of GM across the neuronal populations (conventions are as in Fig. 2b). In PMd (bottom), there was a significant bias toward negative values (mean, -0.08 ; gray triangle; $t$ test) indicating on average stronger antipreference in PMd, but not in PRR (top; white triangle). The GM distributions in both areas were broader than for the shuffled data (dashed line; $\sigma_{\mathrm{d}}>\sigma_{s^{\prime}}$ Bartlett's test) indicating significant modulation effects in both areas. Significance levels were as follows: ${ }^{*} p<0.05,{ }^{* *} p<0.01,{ }^{* * *} p<0.001$.

Materials and Methods and supplemental material S1, available at www.jneurosci.org). Figure $3 a$ shows two example neurons that were gain modulated by the behavioral context. The first neuron (top) was highly active in pro-trials, but responded only weakly in anti-trials. The preferred direction, representing the motor goal, remained unchanged (DD not significantly different from $180^{\circ}$ ), as did the directional selectivity (SM not different from zero). The second neuron (bottom row) showed the opposite preference and responded strongly in anti-trials, but only weakly in pro-trials. Again the spatial tuning encoded the motor goal in both rule conditions in a similar manner. Twenty-one percent (43 of 205) of neurons in PRR and 37\% (49 of 132) in PMd had a significant GM when tested in individual neurons (Fig. 3b). There was a significant difference between the numbers of significant pro-preferring and anti-preferring neurons in PRR (31 pro, 12 anti; $p=3.7 \times 10^{-3} ; \chi^{2}$ test) but not in PMd (28:21; $p>0.05$ ) (for the number ratios, see Fig. $6 c$ ). On the population level, in both areas the GM values were more broadly distributed (i.e., on average, had higher absolute values) than the GM of the shuffled data (PRR, $p<10^{-10}$; PMd, $p<10^{-10}$; Bartlett's test), indicating contextual gain modulation (Fig. 3b) (see also Fig. 6a). In PMd, the GM distribution additionally had a significant bias toward anti-preferring neurons $\left(m=-0.08 ; p=8 \times 10^{-4} ; t\right.$ test) (i.e., neurons in PMd were on average more active during the planning of anti-reaches). In PRR, the GM distribution was centered on zero $(m=0.021 ; p>0.05 ; t$ test) (see also Fig. $6 b)$.

\section{Contextual modulations of directional selectivity}

In addition to a modulatory effect on the gain of the motor-goal tuning, context also modulated the directional selectivity of many neurons in our experiment (Fig. 4). In the first example (Fig. 4a, top), the tuning of the neuron, which was highly selective for pro-reaches to the left, became bimodal in the case of antireaches (i.e., was active for leftward and rightward anti-reaches). The second example (Fig. 4a, bottom) shows another neuron that was spatially tuned in pro-trials but not tuned in anti-trials. Twenty-six percent (54 of 205) of neurons in PRR and 25\% (33 of 132 ) in PMd had a significant SM (Fig. 4b). There was a signifi- cant difference between the numbers of significant propreferring and anti-preferring neurons in PRR (45 pro, 9 anti; $p=3.2 \times 10^{-7} ; \chi^{2}$ test) but not in PMd (18:15; $\left.p>0.05\right)$ (see also Fig. $6 c$ ). Correspondingly, the population distribution of SM (Fig. $4 b)$ in PRR shows a shift toward positive values $(m=0.11 ; p=2.0 \times$ $10^{-7} ; t$ test), indicating a bias toward greater directional selectivity in pro-trials than in anti-trials (see also Fig. 6b). In PMd, there was no shift of the population mean toward pro-preferring or antipreferring selectivity $(m=0.02 ; p>0.05 ; t$ test). SMs were more widely distributed in the original data than in the shuffled data in both areas (Bartlett's test: PRR, $p=2.4 \times 10^{-4}$; PMd, $p=4.7 \times$ $\left.10^{-6}\right)$, indicating significantly stronger modulation of tuning selectivity than expected by chance (see also Fig. $6 a$ ). The SM values in PMd had a trend to be bimodally distributed, indicating simultaneous presence of neurons with rather strong modulation of directional selectivity preferring either pro-reaches or anti-reaches.

\section{Direct context modulation}

Modulation of motor-goal tuning in $\mathrm{PMd}$ and $\mathrm{PRR}$ requires that the contextual information about the currently valid transformation rule is accessible to these cortical regions. How strongly is this transformation rule represented in PRR and PMd neurons at a time when only the rule (pro/anti) and not the complete motor goal is known to the monkey (i.e., independent of any spatial representations)? The direct context modulation index (DM) compares the level of neuronal activity during the memory period in the $\mathrm{R}$ condition, when contextual but not spatial information is available. Sixteen percent (42 of 258) of neurons in PRR and 25\% (49 of 193) in PMd had a significant DM (Fig. 5). There was no significant difference between the numbers of significant pro-preferring and anti-preferring neurons in either PRR (24 pro, 18 anti; $p>0.05 ; \chi^{2}$ test) or PMd (23:26; $\left.p>0.05\right)$ (Fig. $6 c$ ). The balance between propreference and antipreference was also reflected in the fact that the distributions of DM values were centered at zero in PRR (mean $m=0.011 ; p>0.05 ; t$ test) and PMd ( $m=0.013 ; p>0.05$ ) (Fig. $6 b)$. The variances of the DM distributions were significantly larger than the variances of the 
a Pro selective cell (bimodal in anti) $\mathrm{SM}=0.73, \mathrm{GM}=-0.06($ PRR, monkey $A)$
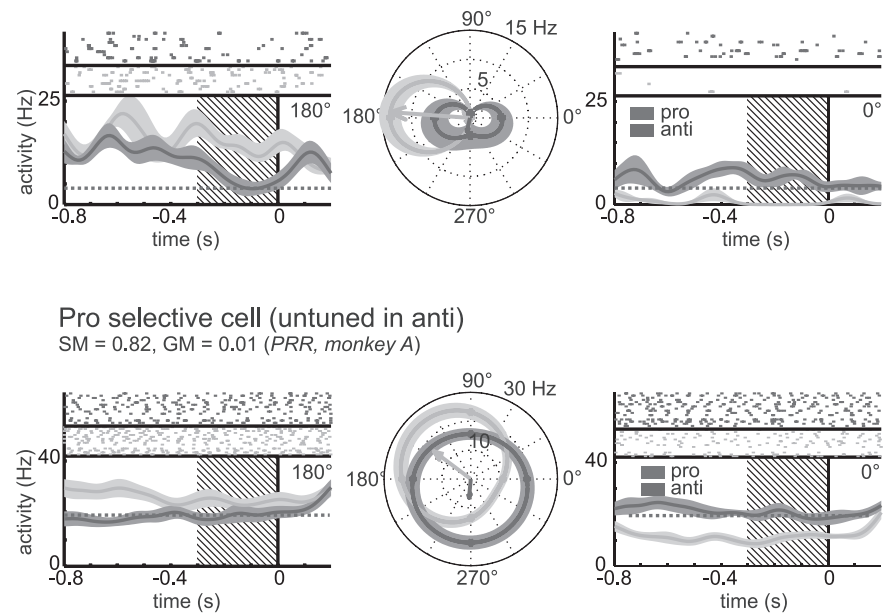

ned in anti)
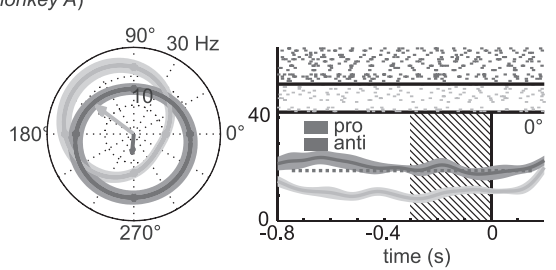

b

Selectivity modulation (SM)

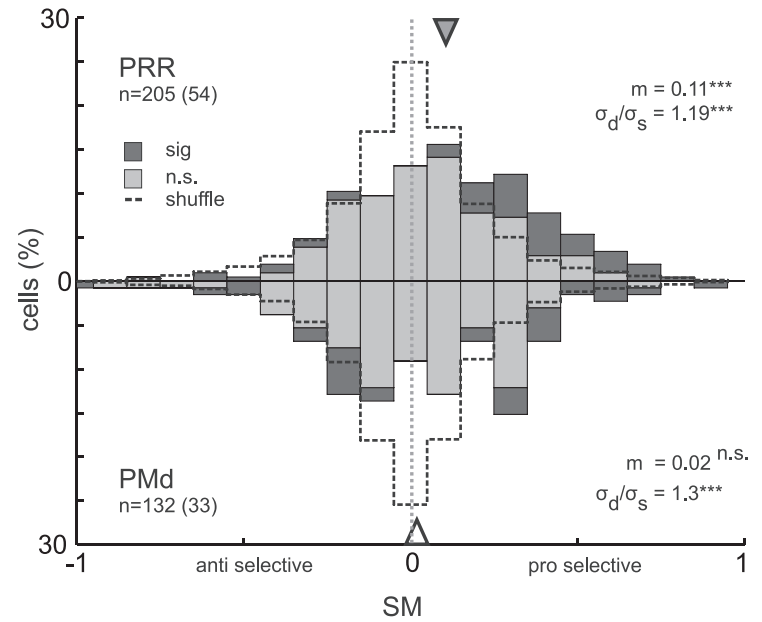

Figure 4. Contextual selectivity modulation of motor-goal tuning in PRR and PMd. $\boldsymbol{a}$, Examples of cells with a high absolute SM, indicating stronger directional selectivity for either pro-reaches or anti-reaches, but having the same response strength for both conditions on average across all reach directions (GM not significantly different from zero; conventions are as in Fig. 2a). The modulation resulted in different forms of tuning [e.g., bimodal (top) or nonselective (bottom) tuning functions during anti-reach planning]. $\boldsymbol{b}$, Distribution of SM across the neuronal populations (conventions are as in Fig. 2b). There was a significant bias for positive (pro-preferring) values in PRR (top), but not in PMd (bottom), indicating on average stronger directional selectivity during pro-reach planning in PRR, like the examples shown in $\boldsymbol{a}$. The SM distributions in both areas were broader than those of the shuffled data (dashed line; $\sigma_{\mathrm{d}}>\sigma_{\mathrm{s}^{\prime}}$ Bartlett's test) indicating significant modulation effects in both areas (conventions are as in Fig. 3).

shuffled DM data in both areas (PRR, $p=9.1 \times 10^{-4} ; \mathrm{PMd}, p=$ $1.1 \times 10^{-7}$; Bartlett's test) (Fig. $6 a$ ).

\section{Complementary contextual modulations in PRR and PMd}

Figure 6 summarizes the contextual modulation effects on the gain of tuned neural responses (GM) and their directional selectivity (SM), together with the direct neural responses to the context information itself (DM). When compared with the shuffle prediction, contextual gain modulation showed the strongest effect, both in PRR and PMd (Fig. 6a). Yet directional selectivity modulation and direct contextual modulation indices were also significantly larger than expected by chance in both areas. The bias of the contextual modulation effects in terms of a propreference/antipreference were complementary in the two areas (Fig. 6b): Contextual gain modulations in PMd were biased toward antipreference [i.e., tuned responses were on average $15 \%$ $(\mathrm{GM}=-0.08)$ higher during anti-reaches compared with proreaches, whereas in PRR gain modulations on average were balanced]. In contrast, directional selectivity modulations in PRR were biased toward propreference; i.e., spatial tuning was on average stronger in pro-reaches compared with anti-reaches by $20 \%$ ( SM = 0.11), whereas in PMd, selectivity modulations on average were balanced. In PRR, the fraction of individual neurons being biased toward higher response amplitudes or stronger directional selectivity was larger in pro-reaches compared with anti-reaches, whereas in PMd the number of individually modulated neurons was balanced (Fig. 6c).

\section{Different types of modulation in different neuronal populations?}

The contextual gain and selectivity modulation indices (GM and $\mathrm{SM}$ ) per se are independent of each other (supplemental material S1, available at www.jneurosci.org). But one could imagine that, in individual neurons, the different modulation effects are correlated. This would be the case if two types of modulation are the phenomenological consequences of one and the same underlying computational mechanism, or if the underlying processes causing the different types of modulation interfere with each other. There was no correlation in either PRR or in PMd between GM and DM $\left(r_{\mathrm{PRR}}=0.1, p_{\mathrm{PRR}}>0.05 ; r_{\mathrm{PMd}}=0.03, p_{\mathrm{PMd}}>0.05\right), \mathrm{SM}$ and DM $\left(r_{\mathrm{PRR}}=0.13, p_{\mathrm{PRR}}>0.05 ; r_{\mathrm{PMd}}=0.07, p_{\mathrm{PMd}}>0.05\right)$, or $\mathrm{SM}$ and $\mathrm{GM}\left(r_{\mathrm{PRR}}=0.06, p_{\mathrm{PRR}}>0.05 ; r_{\mathrm{PMd}}=0.03, p_{\mathrm{PMd}}>0.05\right)$ (Fig. $7 a-c$ ).

Two reasons could account for a lack of correlation between GM, SM, and DM. Either there was no interaction between the underlying processes that caused the gain and the selectivity modulations, but they still occurred within the same neurons; or these processes affected complementary neuronal subpopulations. If different neuronal subpopulations were affected by contextual gain and selectivity modulations, then high absolute values in GM and DM should be paired with low absolute values of the SM, and vice versa. In PRR, absolute values of SM were negatively correlated with absolute values of both GM $(r=-0.3$; $p=0.005$; Spearman's rank correlation $)$ and DM $(r=-0.32 ; p=$ 0.003 ) (Fig. $7 d-f$ ). In PMd, only SM and DM were negatively correlated $(r=-0.36 ; p=0.006)$.

High absolute GM values do not necessarily mean that the neurons have to be gain modulated in an "ideal" way (i.e., in a multiplicative manner, as depicted in the example of Fig. $3 a$ ). Neurons with substantial GM might also have a high absolute SM value, which means, in principle, they might not have the typical unimodal directional tuning. In fact, a high absolute GM indicates a weak response of the neuron in either pro-reaches or anti-reaches. This can lead to weak or undefined directional tuning in the nonresponsive condition, and hence to high SM values. The lack of correlation between GM and SM, together with the anticorrelation of absolute GM and SM values in PRR, indicates that such interdependencies of GM and SM were not very common. To identify those cells that fulfilled all criteria for an ideal contextual gain modulation, we used an additional strict set of constraints that is compatible with the idea of gain modulation proper. We found that $48 \%$ (44 of 92) of cells that had a signifi- 
cant GM were (1) tuned in both context conditions, but did not have a significant SM and did not show a change in preferred direction, or (2) were only tuned in the corresponding context condition (i.e., in the pro condition if they had a positive $\mathrm{GM}$, or in the anti conditions if they had a negative GM) (PRR, 26 of 43, 60\%; PMd, 18 of $49,37 \%$ ).

\section{Discussion}

We found strong modulation effects of behavioral context on the predominant motor-goal tuning in PRR and PMd. We propose gain modulation as a mechanism to achieve flexible goal-directed visuomotor remapping in a context-specific manner. Second, we found different types of modulation with complementary biases in PRR and PMd. PRR showed stronger directional selectivity during the planning of reaches toward directly cued (pro) compared with inferred (anti) goals. PMd, however, showed stronger overall activity during reaches toward inferred compared with directly cued goals.

\section{Contextual modulations in PRR and PMd}

Behavioral context affected neural activity in PRR and PMd in two major ways, either by directly driving neurons (direct context modulation, DM) or by modulating spatial motor-goal representations (contextual gain/selectivity modulations, GM/ $\mathrm{SM}$ ). Both types of context modulation can be predicted from the hidden-layer properties of a neural network model (Brozović et al., 2007). Gain-field modulation evolved in this model as a consequence of learning context-specific spatial cue-response mapping, equivalent to our anti-reach task. Gain modulation of spatially selective neurons is the key principle underlying the spatial transformation mechanism in this model, similar to previous models of multisensory integration for spatial reference frame transformations (Zipser and Andersen, 1988; Salinas and Abbott, 1996). Gain modulation during multisensory integration for eye or hand movements had previously been found in the posterior parietal cortex (Andersen et al., 1985; Brotchie et al., 1995; Galletti et al., 1995; Snyder et al., 1998; Batista et al., 1999; Nakamura et al., 1999; Buneo et al., 2002) and frontal areas (Boussaoud et al., 1993, 1998; Mushiake et al., 1997; Cisek and Kalaska, 2002). In contrast, here we have shown gain modulation effects in PRR and PMd for remapping visuospatial information onto reach motor goals according to abstract cognitive transformation rules. a
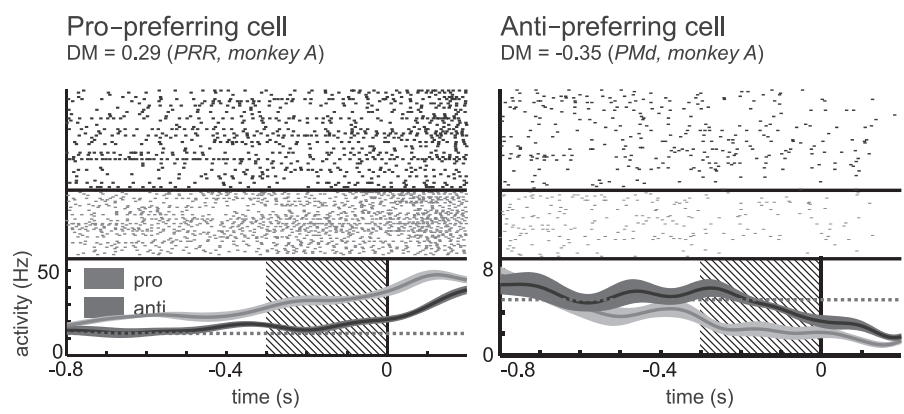

b

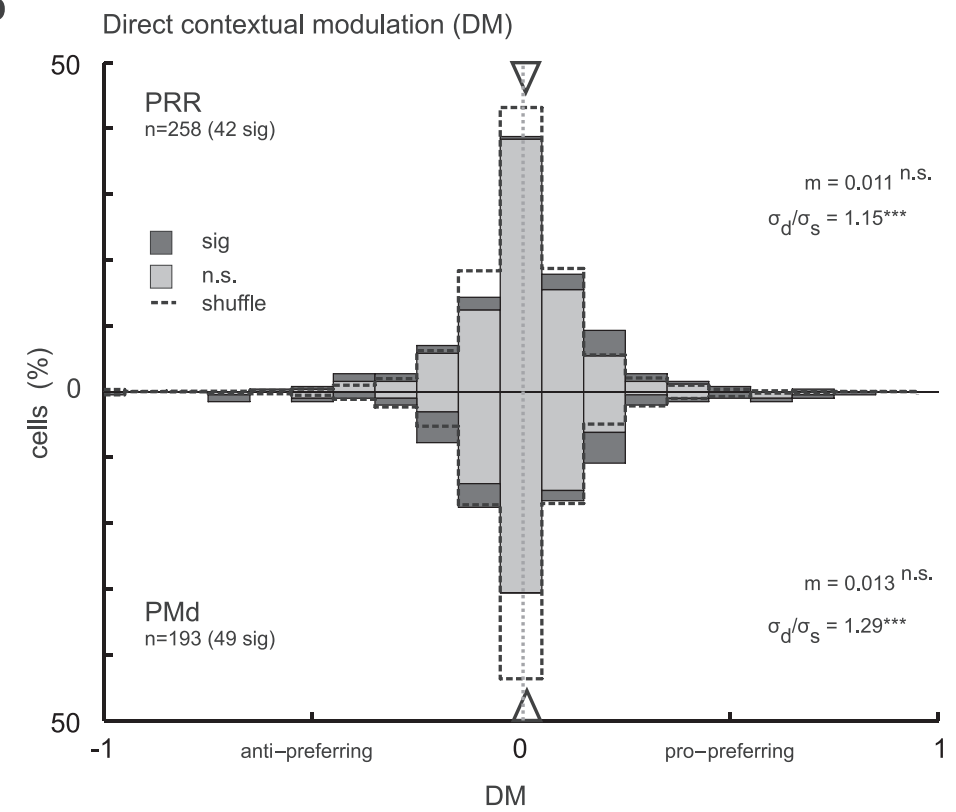

Figure 5. Direct context modulation in PRR and PMd independent of spatial tuning. $\boldsymbol{a}$, Examples show a pro-preferring cell (left), which was continuously more active in the memory period of pro-trials than during anti-trials ( $R$ condition). In the antipreferring cell (right), the contextual modulation appeared later (i.e., only $\sim 500 \mathrm{~ms}$ before the go cue). $\boldsymbol{b}$, Distribution of DM across the neuron populations. In PRR (top) as in PMd (bottom), the distributions were unbiased (i.e., centered at zero). The distribution of DM in both areas was broader than that of the shuffled data (dashed line; $\sigma_{\mathrm{d}}>\sigma_{\mathrm{s}^{\prime}}$ Bartlett's test) indicating contextual modulation effects (all conventions are as in Fig. 3).
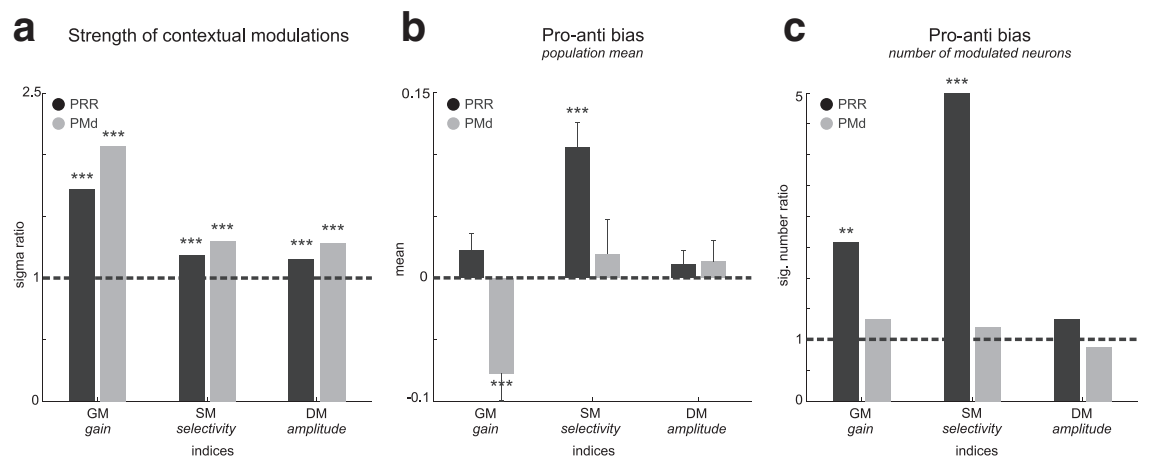

Figure 6. Summary of the strength and bias of contextual modulations in PRR (black) and PMd (gray). $\boldsymbol{a}$, Strength of contextual modulations. Both areas showed contextual modulations of all three types (GM, SM, and DM; Bartlett's test; significance levels are as in Fig. 3), with GM being the strongest. The $\sigma$ ratio indicates the width of the distribution of modulation indices relative to the width of the distribution of the shuffled data (see Materials and Methods) (Figs. 3-5). $\boldsymbol{b}$, Pro/anti bias in the population mean. PRR and PMd showed complementary pro/anti biases in the mean contextual gain and selectivity modulation (GM and SM; mean \pm SEM; $t$ test). $c$, Pro/anti bias in the number of modulated neurons. The relative number of neurons with a propreference versus an antipreference was higher in PRR for GM and SM, but balanced otherwise $\left(\chi^{2}\right.$ test). 
a
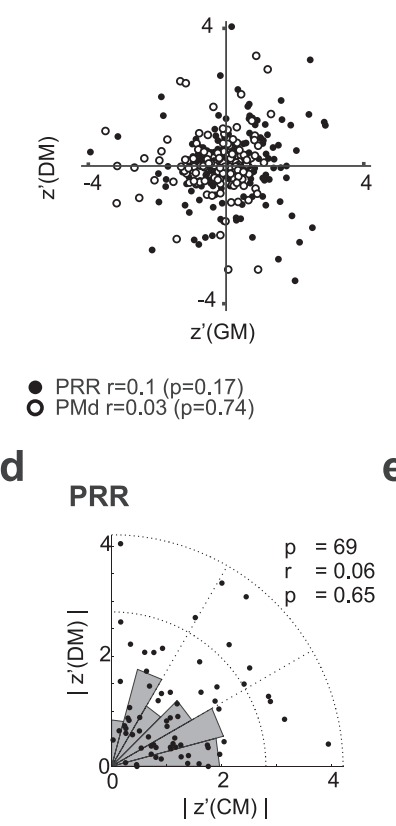

PMd

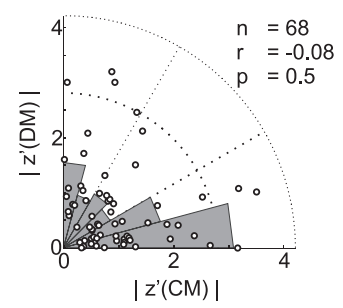

b

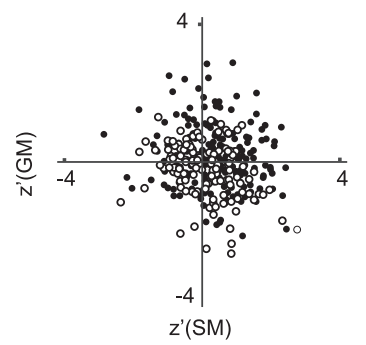

PRR $r=-0.13(p=0.07)$
PMd $r=-0.07(p=0.42)$

e
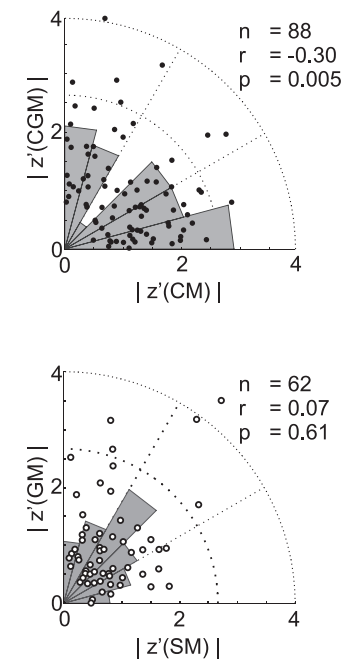

C

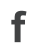

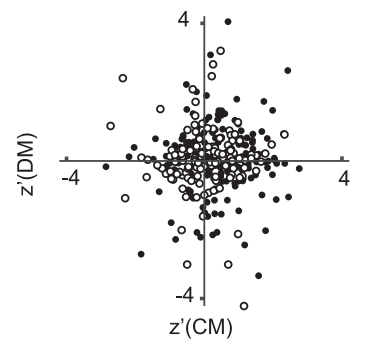

PRR $r=0.06(p=0.4)$
PMd $r=-0.03(p=0.7)$
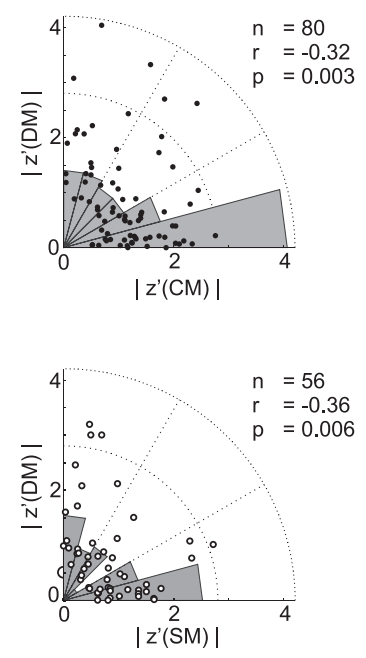

Figure 7. Interdependence of contextual modulations in PRR and PMd. $\boldsymbol{a}-\boldsymbol{c}$, Pairwise cross-correlations between the three modulation indices. The Pearson correlation of the three modulation indices ( $r$ and $p$ values given at the bottom of the diagrams) across neurons shows that none of the indices in either PRR or PMd were significantly correlated. $\boldsymbol{d}-\boldsymbol{f}$, Pairwise cross-correlation of the absolute values of the modulation indices for PRR (top) and PMd (bottom) separately. In PRR, SM-DM (f; top) and SM-GM ( $\boldsymbol{e}$; top) were negatively correlated [i.e., strong GM and DM modulations implied weak SM modulations, and vice versa (Spearman's rank correlation)]. In PMd, only SM and DM showed negative correlations $(\boldsymbol{f})$. The underlying rose plots (gray circular histograms) illustrate the frequencies of relative modulation strengths.

Gain modulation of neurons with motor-goal tuning was partly accompanied by modulation of the directional selectivity. This is consistent with the idea that a contextual gain modulation network builds the computational basis for the remapping process. Gain modulation then does not imply multiplicative modulations in the strict mathematical sense, but rather a summation of bell-shaped tuning functions (directional tuning) with a gain function (contextual tuning), which is passed through a recurrent network with nonlinear transfer properties (Salinas and Abbott, 1996; Brozović et al., 2007, 2008). Such a computational architecture results in gain modulations, which may not only affect the amplitude but also the shape of the tuning functions. This can be observed to varying extend for neurons within the same sensorimotor layer (Brozović et al., 2007).

Direct context modulation, as observed during the R condition, is also very plausible in the light of a gain modulation network. It is predicted for the sensorimotor ("hidden") layer of the network model, when only contextual information but no spatial information is available (Brozović et al., 2007), and all inputs are a priori additive. The impact of the context input in directly driving the neurons might be relatively weak, and only unfold to its full modulatory potential when the neuron is additionally

driven by strong spatial input (Abbott and Chance, 2005). Because of their latency (Fig. 5, right example), we do not attribute the direct context modulations to the sensory properties of the context cue, but rather to the behavioral relevance of the cue (Boussaoud and Wise, 1993; Snyder et al., 1997; Toth and Assad, 2002; Stoet and Snyder, 2004; Nakayama et al., 2008).

Neurons in PMd had previously been reported to be modulated by context, since task rules had an effect on spatial selectivity (di Pellegrino and Wise, 1993; Crammond and Kalaska, 1994). Spatially selective responses were shown to be dependent on whether the motor goal was at the same position as the visual cue or at a default location (di Pellegrino and Wise, 1993). In contrast to our data, the strong condition dependency in the study of di Pellegrino and Wise is attributable to the fact that, in the default condition, the visual cue did not contain any relevant spatial information. Hence the modulation did not reflect an effect of contextualspecific spatial remapping, but rather an effect of motor planning based on spatial remapping versus nonspatial stimulusresponse associations (Crammond and Kalaska, 2000). In contrast, in our experiment, the visual cue location was always relevant for the reach direction and the context modulations are the consequence of two different spatial transformation rules. Crammond and Kalaska reported a substantial fraction of cells in PMd with a significant interaction effect of a remapping rule on the reach-related response during the late phase of an instructed delay period (Crammond and Kalaska, 1994). Their finding could reflect a similar gain mechanism as observed here. However, they could not analyze specific effects of context on gain or selectivity of directional tuning because of their experimental protocol. Also, both previous studies could not compare the effects in PMd with PRR, in which gain modulation effects on directional tuning by spatial transformation rules, to our knowledge, have not been shown before.

\section{Differences between PRR and PMd}

Spatial motor-goal tuning in PRR and PMd, in part, was differently affected by contextual modulations. First, PMd neurons were on average stronger active by $15 \%$ during planning of antireaches, but only if spatial information was already available, i.e., in the RS but not in the R condition, negative bias of GM, but not DM. We interpret this as an indication for the need of overruling a "default" movement plan (pro-reach) induced by the visual cue (Schlag-Rey et al., 1997; Everling et al., 1999), which might otherwise dominate. The stronger activity in PMd during antireaches in this view reflects the learned counterbalance of the network, which is necessary to compensate for the imbalance between pro-reaches and anti-reaches in terms of cue-response congruency. The counterbalance is only necessary in conditions 
in which a spatial cue was already presented, to suppress a default pro-movement (RS condition), but not when only the rule is known and no specific default plan yet exists (R condition). Enhanced activation because of spatial cue-response incongruence was found previously in the supplementary eye fields during the planning of antisaccades (Schlag-Rey et al., 1997; Amador et al., 2004). Stronger responses during planning of prosaccades compared with antisaccades, however, were found in the posterior parietal area LIP (lateral intraparietal area) (Gottlieb and Goldberg, 1999). We found a higher fraction of individual neurons in PRR with a pro-preferring bias in response strength, but no bias in the average population response.

Second, neurons in PRR were more directionally selective during planning of pro-reaches (positive bias of SM corresponding to 20\% stronger selectivity). This might reflect conflicting input to PRR specifically during planning of anti-reaches. Opposing input could result from bottom-up visuospatial information about the (remembered) cue location or default reach goal, on the one hand, and top-down projections of spatial motor-goal information from motor-tuned output stages, on the other hand (Brozović et al., 2007). Despite predominant motor-goal tuning on the population level (Gail and Andersen, 2006), the combination of such opposing inputs could explain the strongly reduced directional selectivity, which was found in many PRR neurons mainly in the anti-reach condition.

Together, the complementary effects of context on motorgoal representations indicate that posterior parietal cortex might more strongly represent stimulus-driven default or automatic movement plans (Desmurget et al., 1999; Pisella et al., 2000), whereas premotor cortex counterbalances this with predominant representations of rule-guided "inferred" movement plans.

\section{Different contextual modulations in different neuronal populations}

In PRR, those neurons that show strong gain modulation (high absolute GM) tended to have weak directional selectivity modulation (low absolute SM), and vice versa. This suggests that gain and selectivity modulations are the result of two separate underlying mechanisms, which affect different neuronal subpopulations. Gain and direct modulations, on the other hand, did not show such mutual exclusiveness. These findings are consistent with our predictions based on the previous model (Brozović et al., 2007). If the reduced directional selectivity in anti-reaches is indeed a consequence of conflicting independent bottom-up visuospatial and top-down motor-goal input to PRR, then we would not expect the GM/DM versus SM to necessarily be large in the same neurons. In PMd, we could not identify a systematic relationship or correlation pattern between the different types of contextual modulations.

\section{Gain modulation as universal mechanism for flexible remapping}

The contextual gain modulation of motor-goal representations in PMd and PRR strongly suggests gain-field mechanisms for flexibly mapping spatial sensory information onto spatial motorgoal representations according to arbitrary transformation rules. Based on our data, we propose that gain modulation is used by the brain not only in sensorimotor areas to achieve reference frame transformations driven by multisensory input (Andersen et al., 1985; Zipser and Andersen, 1988; Boussaoud et al., 1993, 1998; Brotchie et al., 1995; Galletti et al., 1995; Buneo et al., 2002), but also in the frontoparietal reach network to achieve contextually modulated, goal-directed visuomotor remapping, as previ- ously suggested theoretically (Salinas, 2004; Brozović et al., 2007). Our current data support this idea by providing first experimental evidence for context-specific gain modulations of spatial motor-goal tuning in PRR and PMd, which could denote the key underlying principle of flexible goal-directed behavior.

\section{References}

Abbott LF, Chance FS (2005) Drivers and modulators from push-pull and balanced synaptic input. Prog Brain Res 149:147-155.

Amador N, Schlag-Rey M, Schlag J (2004) Primate antisaccade. II. Supplementary eye field neuronal activity predicts correct performance. J Neurophysiol 91:1672-1689.

Andersen RA, Buneo CA (2002) Intentional maps in posterior parietal cortex. Annu Rev Neurosci 25:189-220.

Andersen RA, Essick GK, Siegel RM (1985) Encoding of spatial location by posterior parietal neurons. Science 230:456-458.

Batista AP, Buneo CA, Snyder LH, Andersen RA (1999) Reach plans in eyecentered coordinates. Science 285:257-260.

Blatt GJ, Andersen RA, Stoner GR (1990) Visual receptive field organization and cortico-cortical connections of the lateral intraparietal area (area LIP) in the macaque. J Comp Neurol 299:421-445.

Boussaoud D, Wise SP (1993) Primate frontal-cortex-effects of stimulus and movement. Exp Brain Res 95:28-40.

Boussaoud D, Barth TM, Wise SP (1993) Effects of gaze on apparent visual responses of frontal-cortex neurons. Exp Brain Res 93:423-434.

Boussaoud D, Jouffrais C, Bremmer F (1998) Eye position effects on the neuronal activity of dorsal premotor cortex in the macaque monkey. J Neurophysiol 80:1132-1150.

Brotchie PR, Andersen RA, Snyder LH, Goodman SJ (1995) Head position signals used by parietal neurons to encode locations of visual stimuli. Nature 375:232-235.

Brozović M, Gail A, Andersen RA (2007) Gain mechanisms for contextually guided visuomotor transformations. J Neurosci 27:10588-10596.

Brozović M, Abbott LF, Andersen RA (2008) Mechanism of gain modulation at single neuron and network levels. J Comput Neurosci 25:158-168.

Buneo CA, Jarvis MR, Batista AP, Andersen RA (2002) Direct visuomotor transformations for reaching. Nature 416:632-636.

Cisek P (2007) Cortical mechanisms of action selection: the affordance competition hypothesis. Philos Trans $\mathrm{R}$ Soc Lond B Biol Sci 362:1585-1599.

Cisek P, Kalaska JF (2002) Modest gaze-related discharge modulation in monkey dorsal premotor cortex during a reaching task performed with free fixation. J Neurophysiol 88:1064-1072.

Colby CL, Gattass R, Olson CR, Gross CG (1988) Topographical organization of cortical afferents to extrastriate visual area PO in the macaque-a dual tracer study. J Comp Neurol 269:392-413.

Crammond DJ, Kalaska JF (1994) Modulation of preparatory neuronal activity in dorsal premotor cortex due to stimulus-response compatibility. J Neurophysiol 71:1281-1284.

Crammond DJ, Kalaska JF (2000) Prior information in motor and premotor cortex: activity during the delay period and effect on pre-movement activity. J Neurophysiol 84:986-1005.

Desmurget M, Epstein CM, Turner RS, Prablanc C, Alexander GE, Grafton ST (1999) Role of the posterior parietal cortex in updating reaching movements to a visual target. Nat Neurosci 2:563-567.

di Pellegrino G, Wise SP (1993) Visuospatial versus visuomotor activity in the premotor and prefrontal cortex of a primate. J Neurosci 13:1227-1243.

Everling S, Dorris MC, Klein RM, Munoz DP (1999) Role of primate superior colliculus in preparation and execution of anti-saccades and prosaccades. J Neurosci 19:2740-2754.

Gail A, Andersen RA (2006) Neural dynamics in monkey parietal reach region reflect context-specific sensorimotor transformations. J Neurosci 26:9376-9384.

Galletti C, Battaglini PP, Fattori P (1995) Eye position influence on the parieto-occipital area PO (V6) of the macaque monkey. Eur J Neurosci 7:2486-2501.

Gottlieb J, Goldberg ME (1999) Activity of neurons in the lateral intraparietal area of the monkey during an antisaccade task. Nat Neurosci 2:906-912.

Kalaska JF (1996) Parietal cortex area 5 and visuomotor behavior. Can J Physiol Pharmacol 74:483-498. 
Marconi B, Genovesio A, Battaglia-Mayer A, Ferraina S, Squatrito S, Molinari M, Lacquaniti F, Caminiti R (2001) Eye-hand coordination during reaching. I. Anatomical relationships between parietal and frontal cortex. Cereb Cortex 11:513-527.

Mushiake H, Tanatsugu Y, Tanji J (1997) Neuronal activity in the ventral part of premotor cortex during target-reach movement is modulated by direction of gaze. J Neurophysiol 78:567-571.

Nakamura K, Chung HH, Graziano MS, Gross CG (1999) Dynamic representation of eye position in the parieto-occipital sulcus. J Neurophysiol 81:2374-2385.

Nakayama Y, Yamagata T, Tanji J, Hoshi E (2008) Transformation of a virtual action plan into a motor plan in the premotor cortex. J Neurosci 28:10287-10297.

Pisella L, Gréa H, Tilikete C, Vighetto A, Desmurget M, Rode G, Boisson D, Rossetti Y (2000) An "automatic pilot" for the hand in human posterior parietal cortex: toward reinterpreting optic ataxia. Nat Neurosci 3:729-736.

Rushworth MF, Nixon PD, Passingham RE (1997) Parietal cortex and movement. 1. Movement selection and reaching. Exp Brain Res 117:292-310.

Salinas E (2004) Fast remapping of sensory stimuli onto motor actions on the basis of contextual modulation. J Neurosci 24:1113-1118.

Salinas E, Abbott LF (1996) A model of multiplicative neural responses in parietal cortex. Proc Natl Acad Sci U S A 93:11956-11961.

Salinas E, Thier P (2000) Gain modulation: a major computational principle of the central nervous system. Neuron 27:15-21.

Schlag-Rey M, Amador N, Sanchez H, Schlag J (1997) Antisaccade perfor- mance predicted by neuronal activity in the supplementary eye field. Nature 390:398-401.

Snyder LH, Batista AP, Andersen RA (1997) Coding of intention in the posterior parietal cortex. Nature 386:167-170.

Snyder LH, Grieve KL, Brotchie P, Andersen RA (1998) Separate body- and world-referenced representations of visual space in parietal cortex. Nature 394:887-891.

Stoet G, Snyder LH (2004) Single neurons in posterior parietal cortex of monkeys encode cognitive set. Neuron 42:1003-1012.

Toni I, Rushworth MF, Passingham RE (2001) Neural correlates of visuomotor associations - spatial rules compared with arbitrary rules. Exp Brain Res 141:359-369.

Toth LJ, Assad JA (2002) Dynamic coding of behaviourally relevant stimuli in parietal cortex. Nature 415:165-168.

Wallis JD, Miller EK (2003) From rule to response: neuronal processes in the premotor and prefrontal cortex. J Neurophysiol 90:1790-1806.

Weinrich M, Wise SP (1982) The premotor cortex of the monkey. J Neurosci 2:1329-1345.

Wise SP, Murray EA (2000) Arbitrary associations between antecedents and actions. Trends Neurosci 23:271-276.

Wise SP, Boussaoud D, Johnson PB, Caminiti R (1997) Premotor and parietal cortex: corticocortical connectivity and combinatorial computations. Annu Rev Neurosci 20:25-42.

Zar JH (1999) Biostatistical analysis. Upper Saddle River, NJ: Prentice Hall.

Zipser D, Andersen RA (1988) A back-propagation programmed network that simulates response properties of a subset of posterior parietal neurons. Nature 331:679-684. 


\section{Supplemental Material}

\section{S1: Relation between GM and SM}

We use the GM and the SM to measure two different types of contextual modulation of directionally tuned cells: decreased (or increased) overall activity for one of the two transformation rules, on the one hand, and reduced (or enhanced) spatial selectivity, on the other hand. To be good indicators for these modulations, the indices should be selective and independent. Figure S1 shows how GM and SM independently change as a function of either tuning amplitude $A$ or width $\kappa$ of idealized von Mises tuning curves. The von Mises distribution is given by:

$$
f(x \mid \mu, \kappa)=\frac{e^{\kappa \cos (x-\mu)}}{2 \pi I_{0}(\kappa)}
$$

where $I_{0}$ is the modified Bessel function of order zero. Figure S1 shows that for an idealized motor-goal cell with identical tuning width in both task conditions, a change of the amplitude in one task condition only results in a change of the GM without influencing the SM. Note that non-normalized tuning vectors are plotted. Normalized tuning vectors in the left half of the graph would have equal lengths for pro and anti. Note also that in case the response completely vanishes for one transformation rule, the SM becomes arbitrarily high even without systematic change in $\kappa$ (far left). With additional random fluctuations, as typical for empirical neural data, this effect of high SM values would have to be expected for GM values smaller but close to one. If, on the other hand, we only modify the tuning width without changing the overall activity (in a normalized von Mises distribution the area under the curve does not change if $\kappa$ is changed), then only the SM changes and the GM remains unchanged. Since empirical neuronal tuning 
functions not necessarily exactly fit von Mises functions in all cases, we decided to use our non-parametric approach. Our modulation indices (GM, SM) reflect contextual modulations of average amplitude and selectivity independently and without the requirement of a certain exact tuning shape. 


\section{S2: Results in individual monkeys}

Population data for the individual monkeys (Fig. S2) show that contextual modulations in both animals are very similar. Due to the similarity we pooled the data for the main manuscript. In both monkeys DCMi distributions were unbiased in PRR ( $\mathrm{S}: \mathrm{m}=0.006, \mathrm{p}$ $=0.69 ; \mathrm{A}: \mathrm{m}=0.014, \mathrm{p}=0.34 ; \mathrm{t}$-test $)$ and $\mathrm{PMd}(\mathrm{S}: \mathrm{m}=0.018, \mathrm{p}=0.44 ; \mathrm{A}: \mathrm{m}=0.010, \mathrm{p}$ $=0.67)$, but broader than the distribution of the shuffled data, except for PRR in monkey S (PRR S: $p=0.17, A: p=0.0022$; PMd S: $p=6.3 \times 10^{-4}, A: p=3.38 \times 10^{-4}$; Bartlett test). CGM distributions were unbiased in PRR ( $\mathrm{S}: \mathrm{m}=0.0026, \mathrm{p}=0.88 ; \mathrm{A}: \mathrm{m}=0.035, \mathrm{p}$ $=0.064 ; \mathrm{t}$-test $)$ and negatively (anti) biased in PMd $(\mathrm{S}: \mathrm{m}=-0.095, \mathrm{p}=0.013 ; \mathrm{A}: \mathrm{m}=-$ $0.064, p=0.025)$ for both animals. In both areas CGM distributions are broader than expected by chance in both monkeys (PRR S: $p=3.1 \times 10^{-4}$, A: $p<10^{-4}$; PMd S: $p<10^{-}$

${ }^{10}$, A: $\mathrm{p}<10^{-10}$; Bartlett test). The CSM distributions consistently showed a positive bias in PRR (S: $\left.\mathrm{m}=0.14, \mathrm{p}=7.56 \times 10^{-5} ; \mathrm{A}: \mathrm{m}=0.086, \mathrm{p}=4.81 \times 10^{-4}\right)$ and no bias in PMd $(\mathrm{S}: \mathrm{m}=-0.028, \mathrm{p}=0.53 ; \mathrm{A}: \mathrm{m}=0.054, \mathrm{p}=0.13)$. Again $\mathrm{CSM}$ distributions in both areas are broader than expected by chance (PRR S: $p=0.0011, A: p=0.032$; PMd $S: p=$ 0.0018, A: $\mathrm{p}=0.0014 ;$ Bartlett test). 


\section{S3: Gain-modulation of motor-goal versus visual tuning}

The idea that visual cue locations are flexibly mapped onto arbitrary motor-goals by gainmodulation mechanisms implies that at the intermediate level of the visuomotor transformation one should expect gain-modulated visual tuning, rather than gainmodulated motor-goal tuning. After all, motor-goal tuning is considered the outcome of the transformation process, not the intermediate representation. Why did we then see gain-modulation of motor-goal tuning in PRR and PMd? The key to understanding this seeming contradiction is the differentiation between a pure feed-forward network, for achieving the desired transformation, compared to a network with top-down feedback. Brozovic et al. (Brozovic et al., 2007) showed that with both network architectures one can perform context-specific visuomotor transformations via gain-modulation. But the feed-forward network developed gain-modulated visual tuning in the 'hidden' sensorimotor layer, while the top-down network produced the same output with gainmodulated motor-goal tuning in the sensorimotor layer. The latter architecture was considered more physiological in the sense that PRR and PMd had been shown to be tuned for the motor-goal during instructed-delay periods (Crammond and Kalaska, 1994;Gail and Andersen, 2006) and connectivity between the posterior parietal cortex and premotor areas is known to be reciprocal.

One can assume a certain delay before the top-down motor-goal projections exert their influence on the spatial tuning in the sensorimotor part of the network. Hence, the predominant motor-goal tuning during the instructed-delay is preceded by a short period of 'visual' tuning (or default motor-goal tuning for the pro-target) during spatial cue 
presentation, both in the model (Brozovic et al., 2007), and in corresponding physiological data (Gail and Andersen, 2006;Zhang et al., 1997;Zhang and Barash, 2000;Zhang and Barash, 2004). Once the transformation is achieved and the output stage of the sensorimotor network shows motor-goal tuning, this motor-goal tuning will be fed back to the intermediate sensorimotor stages and replace the predominant visual tuning quickly with predominant motor-goal tuning, as has been found. The observed gainmodulation of the motor-goal tuning in this view is a reflectance of the general contextinduced gain-modulation at this processing stage, which has its major functional relevance during the transformation process itself, i.e. when visual tuning dynamically changes to motor-goal tuning, but is still visible in the resulting motor-goal tuning itself. Importantly, if the motor-goal tuning constitutes the outcome of a transformation process based on gain-modulation of visual cue representations then we should expect the shortterm visual tuning during cue presentations to be gain-modulated. This was the case in the model (Brozovic et al., 2007). It is technically very difficult to test this prediction in a physiological experiment like our current study. This is because of the following potential confounds: Since the emergence of a motor goal is a dynamic process which is influenced by the sensory input, it is difficult to find a time window that only accounts for visual tuning - gain-modulated or not - and does not also include those dynamical processes which ultimately lead to a motor goal representation. For example, a neurons early response might have smaller amplitude in anti-reaches because the instruction cue was in its response field (e.g., right) but the motor-goal is on the opposite side, i.e. outside the response field. Hence, we have to expect down-regulation of this neurons' response as soon as the transformation process is initiated (Supplementary Fig. 3). The 
exact timing of this is hard to identify (Supplementary Fig. 3 top and bottom), which makes it impossible to judge if any early difference in response amplitude is an expression of a gain-modulated 'visual' response or the consequence of the transformation process itself. Albeit desirable, we could not achieve a readily interpretable analysis of this early trial period equivalently to the more stationary late memory period.

\section{Reference List}

1. Brozovic,M., Gail,A., and Andersen,R.A. (2007). Gain Mechanisms for Contextually Guided Visuomotor Transformations. Journal of Neuroscience 27, 10588-10596.

2. Crammond,D.J. and Kalaska,J.F. (1994). Modulation of preparatory neuronal activity in dorsal premotor cortex due to stimulus-response compatibility. Journal of Neurophysiology 71, 1281-1284.

3. Gail,A. and Andersen,R.A. (2006). Neural Dynamics in Monkey Parietal Reach Region Reflect Context-Specific Sensorimotor Transformations. Journal of Neuroscience 26, 9376-9384.

4. Zhang,J., Riehle,A., Requin,J., and Kornblum,S. (1997). Dynamics of single neuron activity in monkey primary motor cortex related to sensorimotor transformation. $\mathrm{J}$ Neurosci 17, 2227-2246.

5. Zhang,M. and Barash,S. (2000). Neuronal switching of sensorimotor transformations for antisaccades. Nature 408, 971-975.

6. Zhang,M. and Barash,S. (2004). Persistent LIP Activity in Memory Antisaccades: Working Memory For a Sensorimotor Transformation. Journal of Neurophysiology 91, 1424-1441. 

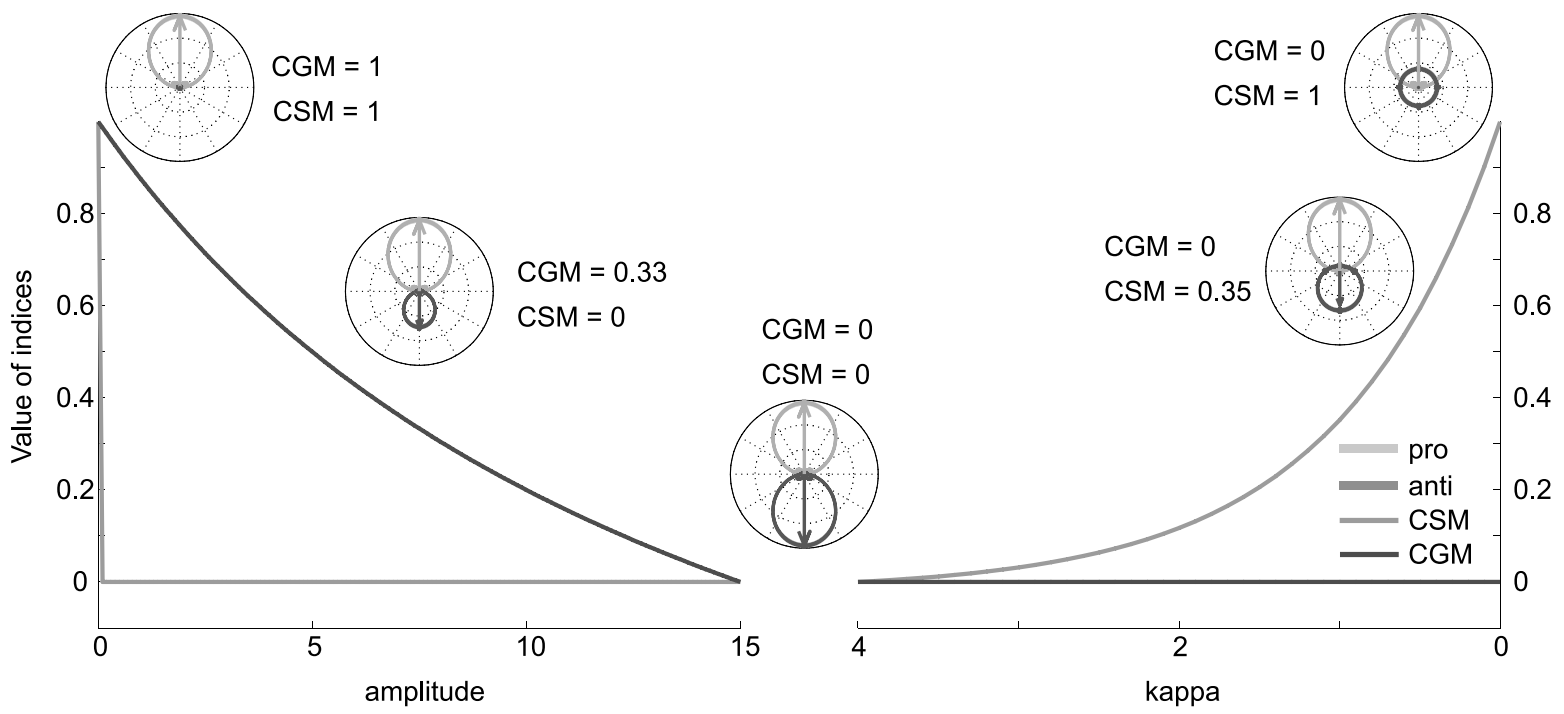

supplementary figure 1 
Monkey A

Monkey S
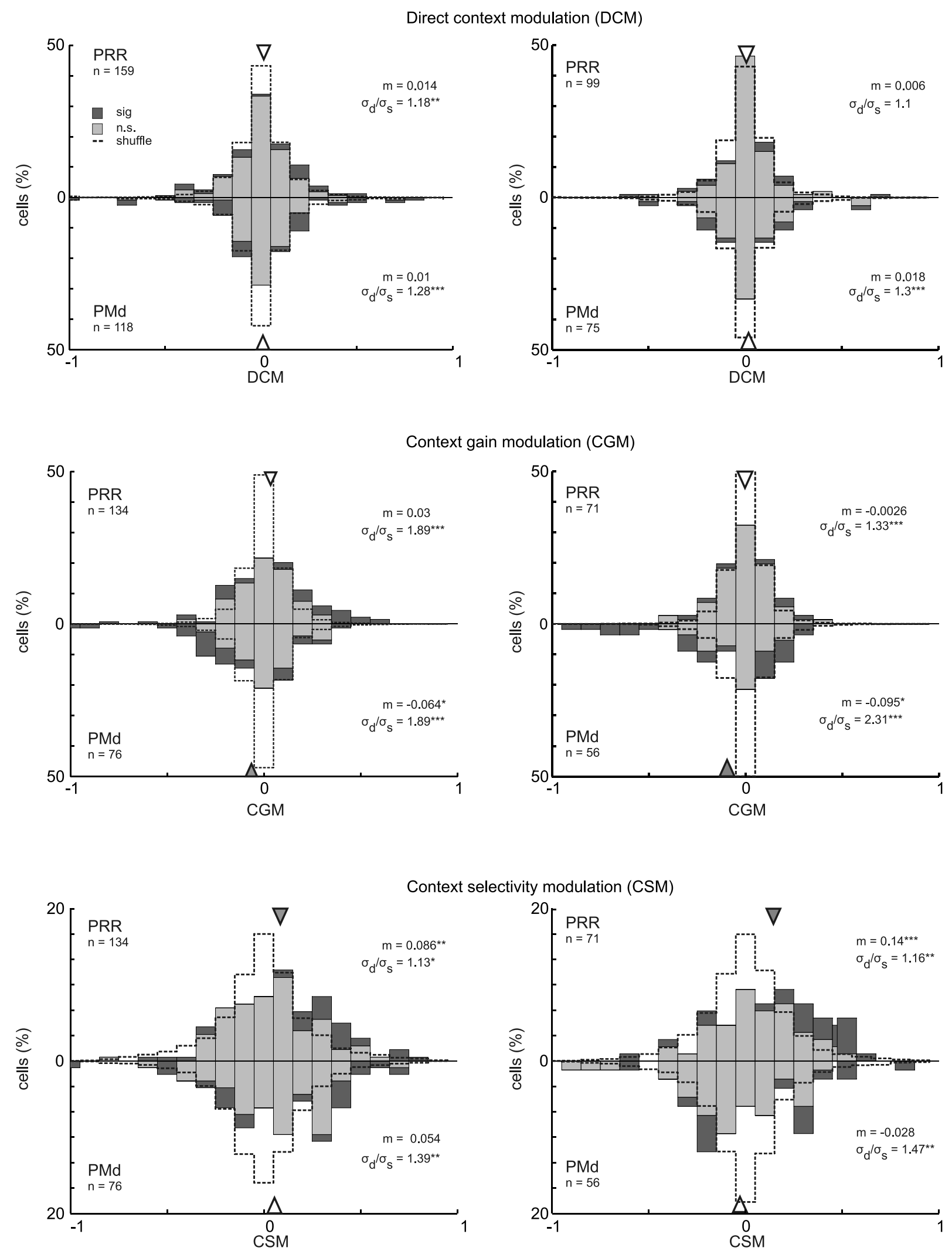

supplementary figure 2 
visual cell example 1

(PMd, monkey A)

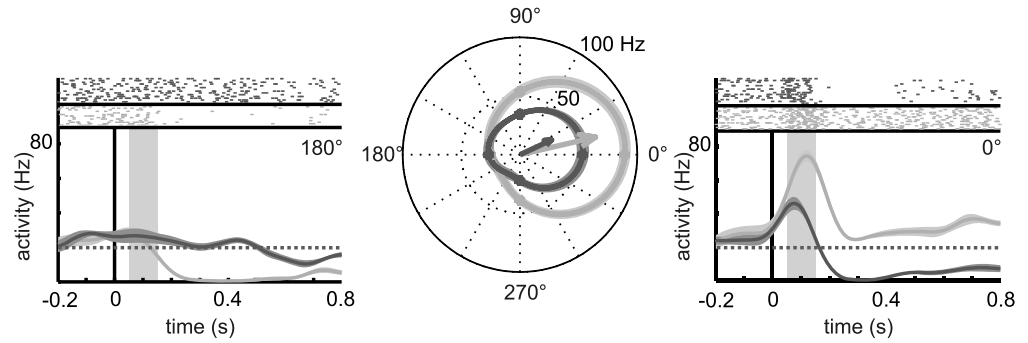

visual cell example 2

(PRR, monkey S)
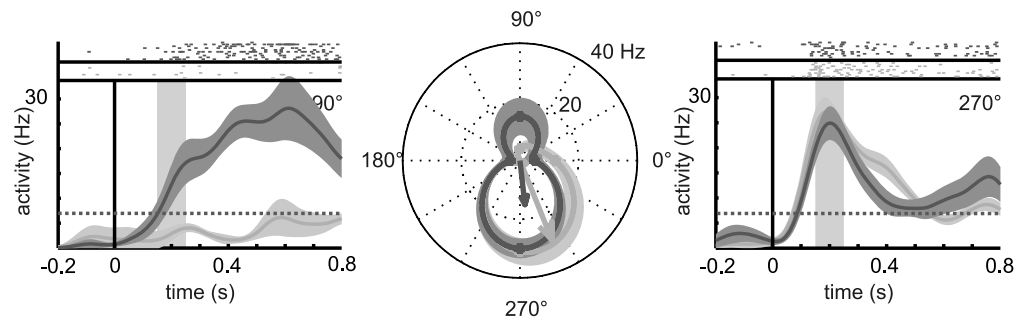

\section{supplementary figure 3}




\title{
The Cortical Timeline for Deciding on Reach Motor Goals
}

\author{
Stephanie Westendorff, Christian Klaes, and Alexander Gail \\ Bernstein Center for Computational Neuroscience, German Primate Center-Leibniz Institute for Primate Research, 37077 Göttingen, Germany
}

Flexible sensorimotor planning is the basis for goal-directed behavior. We investigated the integration of visuospatial information with context-specific transformation rules during reach planning. We were especially interested in the relative timing of motor-goal decisions in monkey dorsal premotor cortex (PMd) and parietal reach region (PRR). We used a rule-based mapping task with different cueing conditions to compare task-dependent motor-goal latencies. The task allowed us a separation of cue-related from motor-related activity, and a separation of activity related to motor planning from activity related to motor initiation or execution. The results show that selectivity for the visuospatial goal of a pending movement occurred earlier in PMd than PRR whenever the task required spatial remapping. Such remapping was needed if the spatial representation of a cue or of a default motor plan had to be transformed into a spatially incongruent representation of the motor goal. In contrast, we did not find frontoparietal latency differences if the spatial representation of the cue or the default plan was spatially congruent with the motor goal. The fact that frontoparietal latency differences occurred only in conditions with spatial remapping was independent of the subjects' partial a priori knowledge about the pending goal. Importantly, frontoparietal latency differences existed for motor-goal representations during movement planning, without immediate motor execution. We interpret our findings as being in support of the hypothesis that latency differences reflect a dynamic reorganization of network activity in PRR, and suggest that the reorganization is contingent on frontoparietal projections from PMd.

\section{Introduction}

Goal-directed behavior is guided by spatial sensory input in combination with context-specific rules. This way, the same sensory input can lead to a wide variety of motor outputs. The reciprocally connected posterior parietal cortex (PPC) and dorsal premotor cortex (PMd) are believed to mediate such space-context integration (Mitz et al., 1991; Kalaska, 1996; Wise et al., 1996; 1997; Kalaska et al., 1997; Passingham et al., 2000; Eliassen et al., 2003; Wallis and Miller, 2003; Stoet and Snyder, 2004; Buneo and Andersen, 2006; Cisek, 2007; Pesaran et al., 2008). For example, neurons in the parietal reach region (PRR) (Gail and Andersen, 2006; Gail et al., 2009) and PMd (Boussaoud and Wise, 1993; di Pellegrino and Wise, 1993; Crammond and Kalaska, 1994, 2000) show sustained motor-goal tuning when the reach goal has to be inferred from a spatial cue by applying a context-specific transformation rule.

How is such space-context integration accomplished, and what is the functional role of reciprocal frontoparietal projections? Network models of space-context integration have suggested frontoparietal projections to help sustain motor-goal memory (Cisek, 2006) or to mediate context-specific visuomotor transformations via gain modulation (Brozović et al., 2007; Gail et al., 2009). Also, frontoparietal neural latency differences (LDs) were shown for motor-related activity around the time of movement initiation (Kalaska et al., 1983; Johnson et al., 1996), which

\footnotetext{
Received Sept. 17, 2009; revised Jan. 22, 2010; accepted Jan. 29, 2010.

This work was supported by German Federal Ministry for Education and Research (Bundesministerium für Bildung und Forschung) Grants 01GQ0433 and 01GQ0814. We thank Sina Plümer and Ludwig Ehrenreich for their help in data collection.

Correspondence should be addressed to Dr. Alexander Gail at the above address. E-mail: agai@@gwdg.de.

DOI:10.1523/JNEUROSCI.4628-09.2010

Copyright $\odot 2010$ the authors $\quad 0270-6474 / 10 / 305426-11 \$ 15.00 / 0$
}

nourished the ideas that frontoparietal LDs could reflect corollary discharge signals (Mountcastle et al., 1975; Bioulac and Lamarre, 1979; Kalaska et al., 1983; Seal and Commenges, 1985; Johnson et al., 1996) and parietal cortex might be involved in internal forward modeling for current state estimation during motor control (Mulliken et al., 2008; Shadmehr and Krakauer, 2008). Finally, for motor planning activity before movement initiation frontoparietal LDs and transient bidirectional frontoparietal interactions are pronounced specifically during decisionmaking tasks, but not for tasks with direct cueing (Pesaran et al., 2008). This suggests that the functional interdependencies between frontal and parietal areas are task dependent (Kalaska and Crammond, 1992; Cisek, 2006; 2007).

We tested how compatible frontoparietal LDs are with the above ideas. We asked specifically whether frontoparietal LDs are the consequence of a corollary discharge signal, and in which way they depend on cognitive decision processes. Different from previous studies, we explicitly isolated the latencies of motor planning activity from cue- or movement-related activity by using a pro-reach/anti-reach task (Crammond and Kalaska, 1994; Zhang et al., 1997; Gail and Andersen, 2006) in combination with partial precueing (Lecas et al., 1986; Riehle and Requin, 1989; Riehle, 1991; Hoshi and Tanji, 2000; Nakayama et al., 2008).

The pattern of frontoparietal LDs that we found in our study suggests that slower motor-goal representations in PRR than PMd reflect a need for the buildup or reorganization of spatial representations in PRR during spatial remapping tasks, independent of corollary discharge or decision signals. We will discuss how far the results could indicate the existence of prospective internal forward model predictions triggered by motor planning rather than by motor execution. 
A

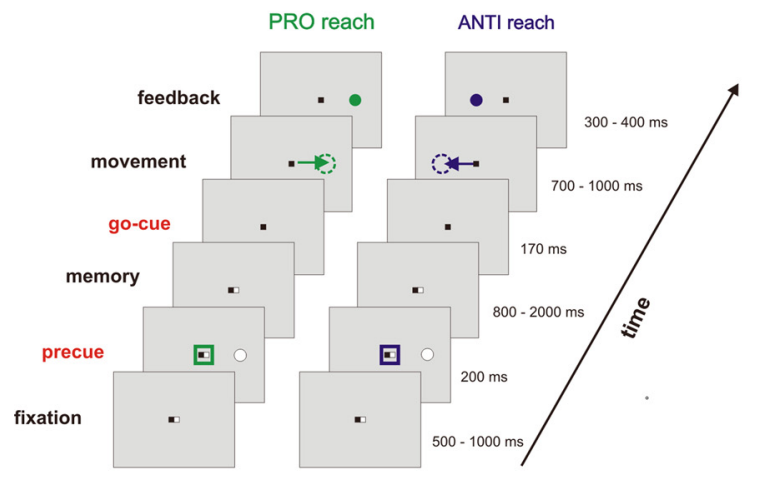

C

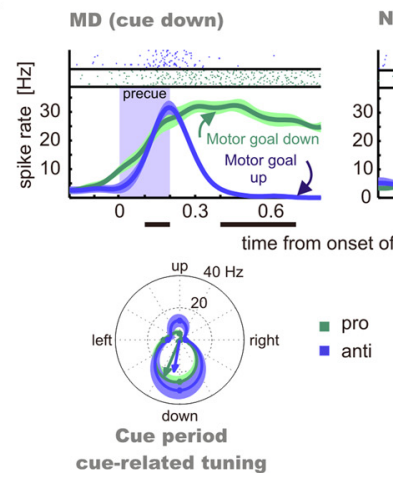

B

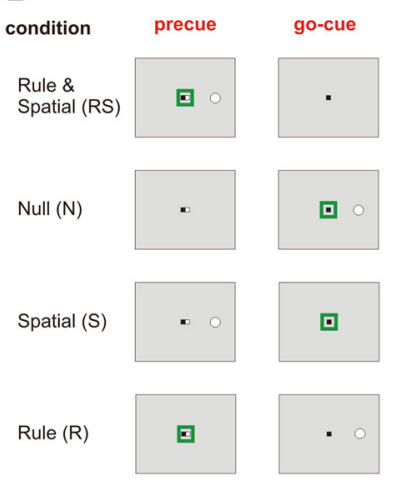

D

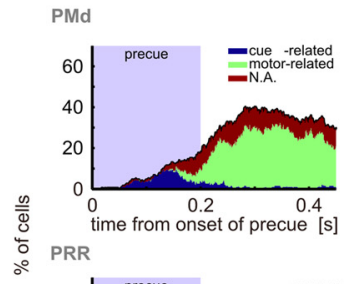

Figure 1. Anti-reach task with different cueing conditions and visuomotor tuning in PMd and PRR. A, Memory-guided antireach task. The left sequence shows an example of a pro-trial, the right an example of an anti-trial in the RS condition. Black and white central squares represent the eye- and hand-fixation stimuli. The central colored frame represents the contextual cue instructing the transformation rule pro or anti, and the peripheral white circle represents the spatial cue. The target position is indicated by the dotted circle. Arrows and dotted circles are for demonstration purpose only and were not shown to the subjects. Note that a go-cue period of $170 \mathrm{~ms}$ is only relevant for other cueing conditions, as in $\boldsymbol{B}$. $\boldsymbol{B}$, In four cueing conditions, the contextual and spatial cue could be presented simultaneously or separately either before or after a variable memory period: transformation rule and spatial cue presented as precue ( $R S$ condition), no precue (N; rule and spatial cue as go-cue), only rule as precue ( $R$; spatial cue as go-cue), or only spatial cue as precue (S; rule as go-cue). C, PSTHs and raster plots of a PRR example neuron. This example motor-goal neuron shows a dynamic switching for cue- to motor-related tuning in response to the precue (shaded area) in the RS condition. Neuronal activities are shown for spatial cues presented at $270^{\circ}$ (left panel; MD) and $90^{\circ}$ (right panel; NP). Polar plots show the directional tuning of the neuron between 100 and $200 \mathrm{~ms}$ (cue period), and 400 and $700 \mathrm{~ms}$ (memory period) relative to the cue position separately for pro- and anti-trials. $\boldsymbol{D}$, Visuomotor dynamics in the population of PMd and PRR neurons. The percentage of spatially tuned neurons of all recorded neurons is plotted for PMd (top) and PRR (bottom) for the first $450 \mathrm{~ms}$ after onset of the precue in the RS condition. Blue bars reflect the percentage of neurons with cue-related tuning, green bars reflect motor-related tuning, and red bars reflect spatially undefined tuning (see Materials and Methods).

\section{Materials and Methods}

Tasks and control of behavior. With our task, we wanted to test how the dynamics of motor-goal decisions and their cortical representations depend on whether the subjects had to perform a memory or reaction-time (RT) task. Further, we aimed to test how partial advance information (precueing) about the to-be-performed reach influences the dynamics of motor-goal decisions.

To separate neural motor-goal representations from other neural signals, we used an anti-reach task design (Gail and Andersen, 2006). The anti-reach task required the subjects to map a spatial instruction cue onto one of two reach goals, either at the location of the cue (pro) or opposite to it (anti). The spatial transformation rule was instructed with a colored context cue (Fig. 1A) (see below for details). The motor goal could only be defined uniquely by the combination of both cues. The eight reach conditions (two context conditions $\times$ four cue directions) were randomly interleaved.

Different cueing conditions were realized by presenting the cues either before (precue period) or after (go-cue period) an instructed delay. The precue consisted of the contextual and the spatial cue together [rule and spatial condition (RS)], only the spatial cue [spatial-only condition (S)], only the contextual cue [rule-only condition $(\mathrm{R})$ ], or nothing [null con-

dition $(\mathrm{N})$ ]. The four cueing conditions were randomly mixed in small blocks of $10-20$ trials (Fig. 1B).

Stimuli were presented on an LCD screen (19 inch ViewSonic LCD VX922; onset latencies corrected). The contextual cue consisted of a colored frame around the small, central red eye and white hand fixation points, and instructed the subject to reach toward (green $=$ pro) or diametrically opposite (blue $=$ anti) of the spatial cue. The peripheral and colorneutral spatial cue was located left, right, above, or below the central fixation points at 9 $\mathrm{cm}$ eccentricity $\left[14.5^{\circ}\right.$ visual angle (VA)].

The exact timeline of the trials was as follows (Fig. 1A): Monkeys initiated the trials by acquiring central eye fixation (tolerance: 2.5-4.0 $0^{\circ} \mathrm{VA} ; 224 \mathrm{~Hz}$ CCD camera, ET-49B, Thomas Recording) and hand fixation at a touch screen (tolerance: $4.0^{\circ} \mathrm{VA}$; IntelliTouch screen, ELO Systems). A variable-length fixation period (500-1000 ms) was followed by the brief precue period $(200 \mathrm{~ms})$. The monkeys had to keep eye and hand fixation for the memory period ( $800-2000 \mathrm{~ms})$, before the hand fixation stimulus disappeared (gosignal). In non-RS trials, a simultaneous gocue was flashed $(170 \mathrm{~ms})$ and completed the missing information to uniquely specify the reach goal. Monkeys had to reach toward the instructed goal within a maximum of 700-1000 ms (movement period; reach tolerance: $\left.5.7^{\circ} \mathrm{VA}\right)$ and hold the goal position for 300-400 ms (feedback period). Visual feedback indicated the correct movement goal (filled circle of the same color as the contextual cue at the goal location) during the last $300 \mathrm{~ms}$ of the feedback period, i.e., only once the monkey had finished the reach. Eye fixation had to be kept throughout the trial. Liquid reward and acoustic feedback indicated correct (high pitch tone, reward) or incorrect (low pitch tone, no reward) behavior.

Animal preparation and neural recordings. Two male rhesus monkeys (Macaca mulatta; monkey $S$ and monkey A) were trained to perform the anti-reach task with partial precueing. The recording procedure was as in previous experiments (Gail et al., 2009). In brief, monkeys were implanted with a titanium head holder and two magnetic resonance imaging (MRI)-compatible recording chambers, custom-fit to the monkeys' heads (3di). Chamber positioning above PRR [Horsley Clarke coordinates: $6 \mathrm{~mm} / 8.5 \mathrm{~mm}$ (monkey S/monkey A) contralateral; $10 \mathrm{~mm} / 9.5 \mathrm{~mm}$ posterior], and $\mathrm{PMd}(13 \mathrm{~mm} / 13.5 \mathrm{~mm}$ contralateral; $17 \mathrm{~mm} / 19.8 \mathrm{~mm}$ anterior) was guided by presurgical structural MRI and confirmed by postsurgical MRI. Sustained directionselective neural responses during center-out reach planning (memory period) were used as a physiological signature in both areas to confirm the region of interest. Both chambers were implanted contralaterally to the handedness of the monkey (monkey A, left hemisphere; monkey S, right hemisphere). All surgical and imaging procedures were conducted under general anesthesia.

We used two five-channel microdrives ("mini-matrix"; Thomas Recording) for extracellular recordings, mostly simultaneously in each chamber. The raw signals of the electrodes were preamplified $(20 \times$; Thomas Recording), bandpass filtered, and amplified ( $154 \mathrm{~Hz}$ to $8.8 \mathrm{kHz}$; 400-800×; Plexon) before online spike sorting was conducted (Sort Client; Plexon). Spike times and spike waveforms were recorded and later subjected to additional offline sorting (Offline Sorter; Plexon). 
Animal care and all experimental procedures were conducted in accordance with German laws governing animal care.

Time-resolved directional and rule selectivity in individual neurons. Time-resolved analysis of directional and rule selectivity was conducted to reveal the cortical dynamics of motor-goal decisions. The analysis time window was between $200 \mathrm{~ms}$ before onset of the relevant cue and $450 \mathrm{~ms}$ after cue onset. In the RS condition, the time around the precue was analyzed. In the N, R, and S conditions, the time around the go-cue was analyzed. All recorded and sufficiently well isolated units were included in the analysis, regardless of their tuning properties, unless explicitly stated otherwise.

For time-continuous analyses, we used spike densities, which were computed by convolving each spike with a causal kernel, which was defined as follows:

$$
R(t)=\frac{\tau_{g}+\tau_{d}}{\tau_{d}^{2}} *\left(1-e^{\frac{-t}{\tau_{g}}}\right) * e^{\frac{-t}{\tau_{d}}},
$$

where $R(t)$ is the spike density at time point $t$. The rise time constant $\tau_{g}$ was set to $2 \mathrm{~ms}$, and the decay time constant $\tau_{d}$ was set to $20 \mathrm{~ms}$ (Thompson et al., 1996; Monosov et al., 2008). Average spike densities across trials with identical conditions (9-15 repetitions per condition) were sampled at $1 \mathrm{~ms}$ resolution and aligned to the onset of either the precue or the go-cue. To reduce variability in the time-resolved tuning analyses of individual neurons (see below), we additionally used a sliding window of $20 \mathrm{~ms}$ length, within which the spike densities were averaged and which was shifted in steps of $1 \mathrm{~ms}$.

Time-resolved rule selectivity (context tuning) was defined by a significant difference (rank sum test) between the average spike densities in pro- and anti-trials in the memory period of the $\mathrm{R}$ condition. The analysis was conducted in each time bin.

Time-resolved directional selectivity was quantified with a directional tuning vector (DTV). It is defined as the vector average across all centerout cue directions $\vec{u}_{i}$ (unit vectors) weighted with the corresponding mean spike rates $r_{i j}$ of neuron $j$ as follows:

$$
\operatorname{DTV}_{j}(t)=\sum_{i=1}^{4} r_{i j}(t) \vec{u}_{i} .
$$

The direction $\alpha$ of the DTV can take any value between $0^{\circ}$ and $360^{\circ}$. The DTV was defined relative to the location of the spatial cue, i.e., $0^{\circ}$ corresponds to a rightward cue. The significance of directional tuning was tested with a nonparametric one-way ANOVA (Kruskal-Wallis, $\alpha=$ 0.05 , unless stated otherwise) plus an additional criterion. The criterion required that the length of the DTV had to be $>0.2$, after the DTV was normalized to the spike density at the direction with the maximal response in that time bin. The fixed criterion of 0.2 was arbitrary, but varying it between 0.15 and 0.25 did not change our conclusions. With this criterion, we excluded neurons with a bimodal tuning close to symmetric. Such symmetric bimodal tuning could represent two potential motor goals instead of one selected motor goal and would be characterized by a significant ANOVA but a DTV length close to zero (see S-4, available at www.jneurosci.org as supplemental material). The DTV was computed in every time bin, separately for pro- and anti-trials, and separately within each cueing condition.

Cue-related versus motor-related tuning. The anti-reach task design allowed separating cue-related from motor-related tuning by comparing the directional selectivity in pro- and anti-reaches (Gail and Andersen, 2006). A neuron's instantaneous (bin-to-bin) directional tuning was categorized according to the absolute difference in DTV direction between pro- and anti-trials in each time bin: cue-related tuning $\left(|\Delta \alpha|<45^{\circ}\right)$, motor-related tuning $\left(|\Delta \alpha|>135^{\circ}\right)$, or spatially undefined tuning (else). This definition of instantaneous tuning depended on a direct bin-wise comparison of spatial tuning between pro- and anti-trials in each cueing condition.

As known from previous studies (Crammond and Kalaska, 1994; Gail and Andersen, 2006), spatiotemporal tuning profiles of individual neurons in PMd and PRR can change over time from cue related to motor related (Fig. 1C), whereas other neurons may show motor-related tuning from the earliest onset of their response. The activity profile of the exam- ple neuron in Figure $1 C$ shows the changing tuning properties of the neuron between the time windows 100-200 ms (cue period) and 400$700 \mathrm{~ms}$ (memory period). Figure $1 D$ shows the number of cue- and motor-related neurons as a fraction of all recorded neurons over the time of the trial in PMd and PRR.

We characterized the neurons according to their spatiotemporal response profiles. We considered neurons to have a significant cue-related response when the instantaneous cue-related tuning in the RS condition persisted in at least $90 \%$ of 30 consecutive time bins within $200 \mathrm{~ms}$ after the onset of the precue (an equivalent definition of cue-related response could be achieved for responses following the go-cue in the $\mathrm{N}$ condition, which did not change our results). We defined neurons as motor-goal neurons when they showed significant movement planning activity. Motor planning activity was defined as motor-related tuning for at least $90 \%$ of 50 consecutive time bins within $450 \mathrm{~ms}$ after precue onset in the RS condition. The different number of time bins for a cue-related response and motor planning activity was set because the cue response is transient, whereas the motor-related response is more sustained. We defined neurons as perimovement neurons that did not show motor planning activity but that at some point in time after the go-cue were motor tuned.

Finally, we defined a time-invariant, motor-related preferred direction (PD) for each neuron. The PD was computed by averaging the tuning vectors in pro-reaches over all time bins of the analysis time window in which the neuron showed significant instantaneous motor-related tuning. The PD was computed separately within each cueing condition.

Determination of motor-goal latencies separately in pro- and anti-trials. If motor-goal latencies in a cortical area were different in pro- and antitrials, then the analysis of instantaneous motor-related tuning would reveal the time course of the motor-goal tuning in the slower of two conditions (pro or anti), since instantaneous tuning is based on an binto-bin comparison between pro- and anti-trials.

To analyze motor-goal latencies separately in pro- and anti-trials, we needed to extract the time courses of cue- versus motor-related tuning in pro- and anti-trials separately. We accomplished this with two measures. First, we restricted our data set to neurons that did not show cue-related responses. Cue-related responses are indistinguishable from motorrelated responses in pro-reaches, since cue and motor goals are spatially identical in this case. Hence, cue-related responses would confound measurements of motor-goal latencies in pro-trials. Second, we compared the instantaneous tuning direction of a neuron in the currently considered context (pro or anti) with the time-invariant PD of this neuron (see definition in the previous section) to decide on the time-resolved cue versus motor relatedness within this context.

Determination of motor-goal latencies in reaction-time conditions. Motor-related tuning could result either from motor planning activity or from perimovement activity (corollary discharge or sensory feedback about the movement). In the RT conditions (N, R, S), the isolation of motor-goal latencies (planning activity) from motor feedback (perimovement activity) was not guaranteed without further precautions. (Only the motor-related tuning in the memory period of the RS condition could be directly considered motor-goal tuning, since movements in these trials were initiated much later.) To isolate motor-goal latencies from latencies of perimovement activity in RT conditions, we separately conducted our latency analyses within either a population of exclusive motorgoal neurons, i.e., neurons that showed significant motor planning activity, or within the complementary population of perimovement neurons.

Population analyses of motor-related tuning. We characterized the motor-related tuning in neural populations in two ways. First, we computed average peristimulus time histograms (PSTHs) across neurons of each cortical area and within each different cueing condition. We did this to illustrate general neural response properties as a function of time. Second, we computed the time-resolved fraction of motor-tuned neurons (recruitment curves) in each cortical area and cueing condition for statistical analyses. We excluded neurons with cue-related responses to show the motor-related time courses.

PSTHs across neurons were computed as the average response in the direction of each neuron's maximal response [PSTH for maximum direction (MD)] and, separately, in the opposite direction [nonpreferred direction (NP)]. The maximum direction for each neuron was defined as 
the direction with the maximal average response across time and trials in pro-trials within the time window of 200-350 ms after cue onset (precue in RS; go-cue in N, R, and S conditions). A time window shorter than the analysis time window was chosen because in PMd the activity of the neurons starts to decrease around $300 \mathrm{~ms}$ after the go-cue (see Fig. 2C,D). Note that the MD and NP directions are restricted to the discrete cue directions left, right, up, and down, since only for these directions PSTHs can be computed. The response of each neuron was normalized to its maximal response within the 200-350 ms time window and within each task condition before averaging across neurons. Average PSTHs across neurons were calculated separately for pro- and anti-trials in MD and NP for each cueing condition. Neurons that did not show significant directional selectivity in any time bin during the analysis time window in either pro- or anti-trials were excluded from this analysis. Because of the necessary normalization, random fluctuations in such nontuned neurons would be inappropriately amplified, and would contribute to the average PSTH with similar weight as strongly tuned neurons.

Recruitment curves denote the fraction of neurons that were motor tuned in a specific time bin relative to the total number of neurons that were motor tuned in any time bin of the analysis time window (see Fig. $2 B$ ). Recruitment curves in our experiment have two advantages over PSTH comparisons. First, normalization and averaging of neural responses were not needed for computing recruitment curves. Averaging across neurons, as done for population PSTHs, typically cannot account for the different response profiles of the individual neurons. For example, many neurons have very low baseline firing and show brisk response onsets after cue presentation, whereas others have high baseline levels and might exhibit their directional selectivity by small excitatory responses to one cue direction and strong inhibitory responses to other directions. Also, normalization tends to boost weak, noisy responses. Second, the recruitment curves are based on tuning functions that interpolate between all four measured directions. The comparison between MD and NP in PSTHs is restricted to two of the four measured directions. As a consequence, PSTHs are less sensitive for neurons with oblique PDs and broad tuning, which is not the case for recruitment curves. For these reasons, we based our statistical analyses on the recruitment curves and use PSTHs only for illustration purposes.

Quantification of neural LDs. We determined motor-goal latencies by a threshold criterion for the neuronal recruitment curves (for controls and alternatives, see S1-S3, available at www.jneurosci.org as supplemental material). Latency of motor tuning within each condition was defined as the average time relative to cue onset at which the fraction of motortuned neurons exceeded each of seven threshold levels for at least $100 \mathrm{~ms}$. Seven equidistant threshold levels were defined relative to the following baseline: $T h(p)=$ baseline $+(p \times(1-$ baseline $))$, where "baseline" refers to the average fraction of tuned neurons in the $200 \mathrm{~ms}$ before cue onset. The $p$ value was set to $0.05,0.1,0.15,0.2,0.25,0.3$, and 0.35 to characterize the early tuning onset of the fastest $\sim 35 \%$ of neurons. The average across seven thresholds was built to become less sensitive to minor random fluctuations. Note that absolute latencies depend on the choice of thresholds, and hence are of limited conclusiveness. We statistically analyzed only latency differences between conditions or neural populations.

Pairwise neural LDs between task conditions or cortical areas were computed and tested for being different from zero. Averaging across absolute latencies measured with multiple threshold levels would induce artificial variance and compromise statistical power. Therefore, we computed the LDs separately for each threshold level and only then computed the average LD across thresholds. Permutation tests $(N=10,000$ samples) were used to estimate the probability that the original sample indicates a significant difference from zero. In each permutation, each neuron's tuning data were randomly assigned to one of the two task conditions or cortical areas in consideration, such that the numbers of neurons in both populations were the same as in the original sample. The resulting $p$ value represents the percentage of random permutations leading to an LD larger or equal to the original sample. A difference was considered significant if the $p$ value was $<5 \%$.

Neural response latencies and behavioral reaction times. Behavioral RTs were defined as the median time from onset of the go-cue until the release of the touch screen within each recording session and task condition. Average RTs were computed across recording sessions. RT differences between pro- and anti-trials were tested with a paired $t$ test across sessions and within each task condition.

To relate motor-goal latencies of individual neurons to behavioral RTs, we analyzed motor-goal latencies after the go-cue in all RT conditions (N, R, and S) on a trial-by-trial basis. We used a threshold criterion to define latencies in those trials where the neurons showed a goodenough response to the pending motor goal: post-go-cue, single-trial activity needed to exceed baseline activity ( = mean spike density of the last $200 \mathrm{~ms}$ of the $\mathrm{N}$ condition memory period) by at least three times its SD for at least $100 \mathrm{~ms}$ within the analysis time window. To cope with the sparse spike events in low-firing neurons, we estimated spike densities in single trials with a Gaussian kernel of width $\sigma=50 \mathrm{~ms}$, as follows:

$$
R(t)=\frac{1}{\sqrt{2 \pi \sigma^{2}}} * e^{\frac{-t^{2}}{2 \sigma^{2}}}
$$

The Gaussian kernel resulted in stronger smoothing than the EPSP kernel used for the trial-average data above. In the RS condition, the onset of motor-goal tuning (after the precue) is not related to the RT (after the gocue); therefore, single-trial latencies in the RS condition were not taken into account. Nontuned neurons and neurons with a cue-related response (see above) were excluded from the analysis to avoid confounds.

We removed RT and single-trial neural latency outliers for the trialto-trial regression analysis between RTs and neural latencies. A value was classified as an outlier if its distance from the median was $>1.5$ times the interquartile range (distance between the 75 th and 25 th percentiles). Since only a small fraction of trials allowed determination of neural response latencies, data from all RT conditions were merged, and linear regression curves were calculated for all neurons in which single trial latencies could be estimated in at least 10 trials.

\section{Results}

Extracellular single-neuron spiking activities were recorded from 258 neurons in PRR (monkey S, 99; monkey A, 159) and 192 neurons in PMd (monkey S, 74; monkey A, 118) while the monkeys performed the anti-reach task with partial precueing (Fig. 1). The average performance of monkey $S$ was $77 \% / 77 \%$ (pro/anti) in the RS condition, $80 \% / 79 \%$ in the $\mathrm{N}$ condition, $75 \% / 75 \%$ in the $\mathrm{R}$ condition, and $79 \% / 79 \%$ in the $\mathrm{S}$ condition. The average performance of monkey A was $86 \% / 86 \%$ in the RS condition, $86 \% / 85 \%$ in the $\mathrm{N}$ condition, $85 \% / 83 \%$ in the $\mathrm{R}$ condition, and $85 \% / 84 \%$ in the $\mathrm{S}$ condition for pro- and anti-reaches, respectively. Errors were mainly caused by ocular fixation breaks and not by incorrect target choices. The choice of the monkey for a reach target was correct in $97 \%$ for monkey S, and in 99\% for monkey A.

\section{Motor-goal latencies after simultaneous cueing of transformation rule and spatial information}

Is motor planning activity represented earlier in either PMd or PRR? If there is an LD, do the LDs depend on a motor command or motor execution, or do they also exist for planning activity proper? To answer these questions, we first analyzed motor-goal tuning latencies in PMd and PRR, when the monkeys were instructed simultaneously with both the transformation rule (pro/anti) and the spatial cue. We compared motor-goal latencies in response to the simultaneous cues in instructed-delay trials (precue in the RS condition) and in reaction-time trials (go-cue in the $\mathrm{N}$ condition). If motorgoal latency differences exist in both conditions - especially if they exist after the precue at the beginning of an instructed delay-then they must be independent of motor execution and represent dynamics of motor planning. If latency differences only existed after a gocue immediately before or during motor execution, then they should be related to corollary discharge or motor-related sensory feedback. The anti-reach task allowed us to separate cue- from motor-related 
PRO

A

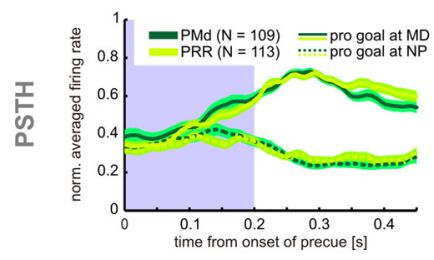

B

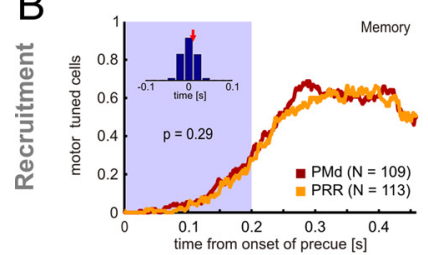

C

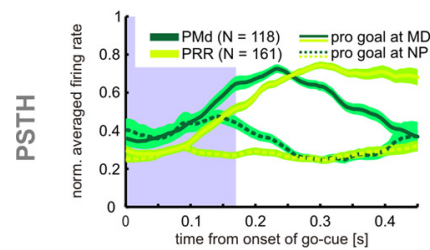

D

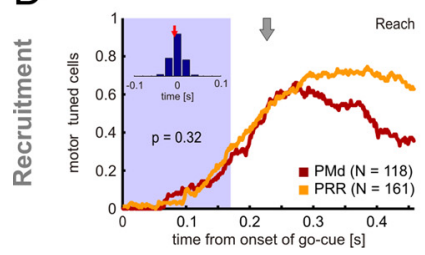

ANTI
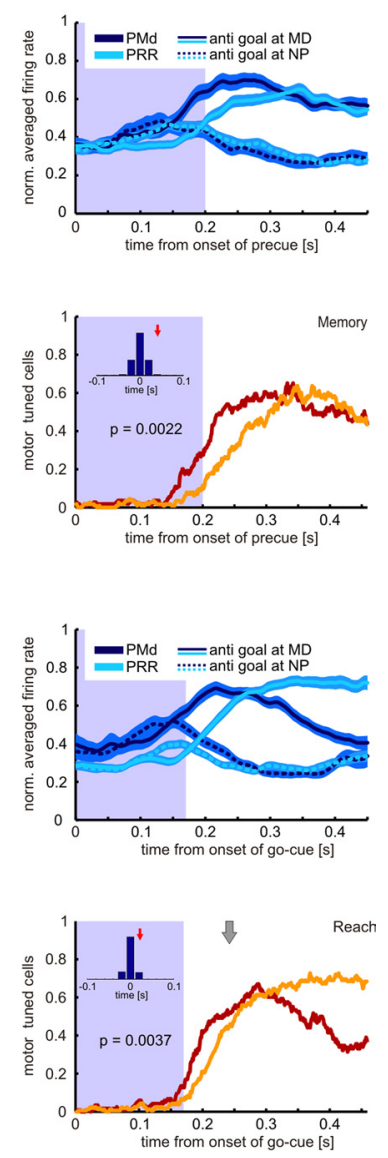

Figure 2. Dynamics of motor-related tuning in PMd and PRR in memory and reaction-time conditions. $\boldsymbol{A}, \boldsymbol{B}$, Data for pro-trials (left column, green) and anti-trials (right column, blue) aligned to the onset of the precue in the RS condition (memory). Shaded areas indicate the time of cue visibility. $\boldsymbol{C}, \boldsymbol{D}$, Equivalent data aligned to the onset of the go-cue in the $\mathrm{N}$ condition (reaction time). Gray arrows in $D$ indicate mean RT across 176 recording sessions. $A, C$, PSTHs: Mean \pm SE (shaded) of PMd (dark colors) and PRR (light colors) normalized population activity in MD (solid) and NP (dotted). $\boldsymbol{B}, \boldsymbol{D}$, Recruitment curves: fraction of neurons tuned in PMd (red) and PRR (yellow) in each time bin relative to the number of neurons tuned for the pro- or anti-goal in any time bin of the analysis time window (numbers in legend). Latencies in each condition were determined via a threshold criterion. Frontoparietal LDs between PMd and PRR were tested with a randomization test (see Materials and Methods). The histogram insets show the distribution of LDs with randomized data compared with the LD of the original sample (red arrow). The $p$ values indicate the significance of the LDs. PMd showed earlier motor-related tuning than PRR in anti-reaches but not in pro-reaches, both in the RS and N condition.

responses (Fig. 1C,D) and to extract motor-goal latencies separately in each context (pro and anti). For analyses of motor-goal latencies separately within each context we had to restrict the neurons to those that did not show cue-related responses (see Materials and Methods). In PMd, 175 of 192 neurons (91\%) fulfilled this criterion. In PRR, this was the case for 215 of 258 neurons ( $83 \%$, RS condition). Because of this necessary constraint, our conclusions-strictly speaking - are limited to neurons without a cue-related response. However, since the fraction of neurons with a cue-related response is small, and only a subpopulation of these would qualify for our analyses because of additional selection criteria, we do not expect that these few neurons would change the results in any significant way.

Figure 2 shows the population PSTHs and fraction of significantly motor-tuned neurons (recruitment curves) in PMd and PRR for pro- and anti-reaches in the RS condition (Fig. $2 A, B$ )

A Rule only (R)
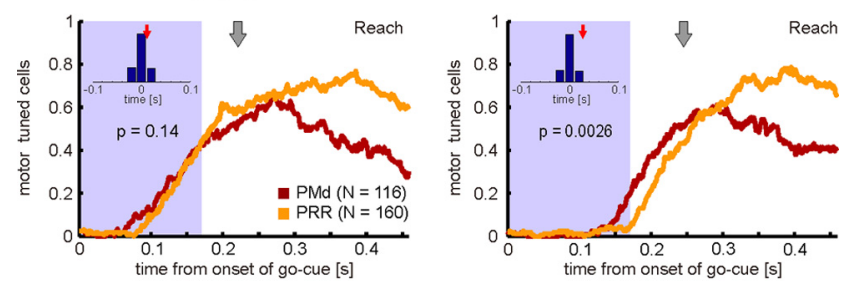

B spatial only (S)
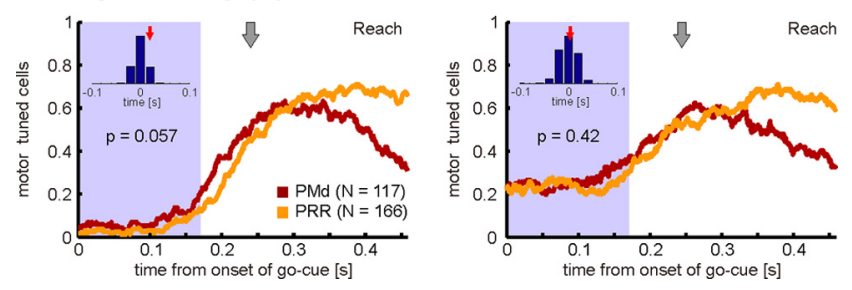

Figure 3. Dynamics of motor-related tuning in conditions with partial precueing. All the conventions for the recruitment curves are as in Figure 2D. $\boldsymbol{A}$, Recruitment curves for the $R$ condition. $\boldsymbol{B}$, Recruitment curves for the $S$ condition. Conditions that required spatial remapping (R-anti, S-pro) showed earlier motor-related tuning in PMd than PRR, whereas conditions without remapping (R-pro, S-anti) showed no LDs. See Results and Figure 5 for details on why S-pro, and not $S$-anti, required spatial remapping from the monkeys. Note that the LD in S-pro was significant when selecting neurons according to a stricter criterion (supplemental Fig. S1, available at www.jneurosci.org as supplemental material).

and $\mathrm{N}$ condition (Fig. 2C,D). In the pro-trials of the RS condition, the population PSTHs in PMd and PRR were practically identical (Fig. $2 \mathrm{~A}$, left). In the anti-trials, on the other hand, the average PRR response to the MD had a higher latency than the PMd response (Fig. 2A, right). Correspondingly, there was no significant LD between PMd and PRR for pro-reaches $(p>0.05$, randomization test), but there was for anti-reaches $\left(\mathrm{LD}_{\mathrm{PRR}-\mathrm{PMd}}=\right.$ $41 \mathrm{~ms}, p=0.0022$ ) in the memory period of the RS condition (Fig. $2 B$ ). In the movement period of the $\mathrm{N}$ condition, the results look similar (for a more in-depth discussion of potential differences, see supplemental Fig. S4, available at www.jneurosci.org as supplemental material). There was no LD between PMd and PRR for pro-reaches, whereas in anti-reaches the motor tuning emerged earlier in PMd than in PRR $\left(\mathrm{LD}_{\mathrm{PRR}-\mathrm{PMd}}=22 \mathrm{~ms}\right.$, $p=0.0037)$. The fact, that the LD is present at the beginning of motor-goal memory trials (RS condition), at a time when the corresponding motor command will be issued at earliest $\sim 1-1.5$ $s$ later, suggests that the LD is related to the decision process about the motor goal or movement planning, rather than to movement execution.

Spatial remapping induces differences in motor-goal latencies between PMd and PRR

In the $\mathrm{N}$ as well as the RS condition, spatial and rule information were presented together, and in both conditions motor-goal tuning was earlier in PMd than PRR, but only for anti-reaches. What factors determine the time course of motor-goal decisions in the frontoparietal network? Is it possible to influence the LDs between PMd and PRR by changing the temporal sequence in which spatial and rule information are provided? We tested the influence of presenting rule information before spatial information $(R$ condition) and of presenting spatial information before rule information (S condition). The results are shown in Figure 3. In the $\mathrm{R}$ condition (Fig. $3 A$ ), there was no frontoparietal LD for progoals ( $p>0.05)$, but for anti-goals motor tuning occurred earlier 


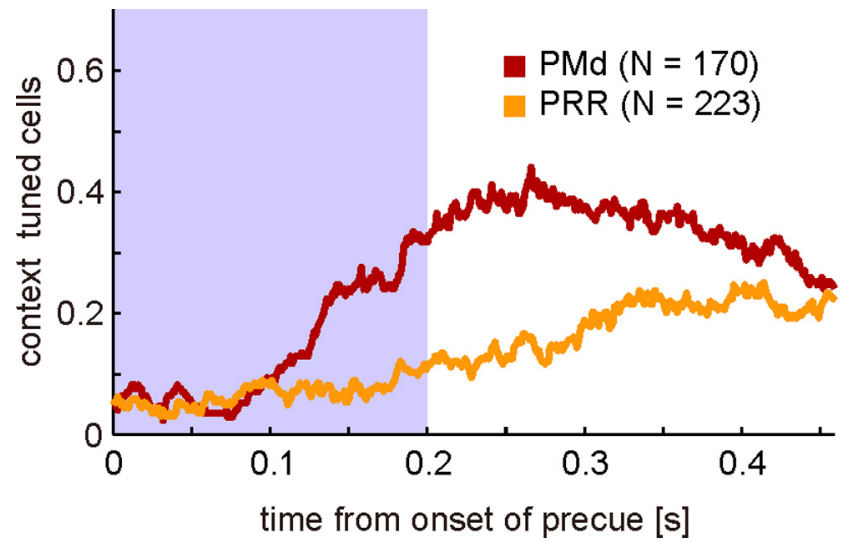

Figure 4. Dynamics of contextual tuning after precueing of the transformation rule. Recruitment curves show that the fraction of neurons with significantly different spike densities between pro-and anti-trials was higher in PMd (red) than PRR (yellow). This was the case especially early in the memory period after the $R$ condition precue. Other conventions are the same as in Figure $2 D$.

in PMd than PRR ( $\left.\mathrm{LD}_{\mathrm{PMd}-\mathrm{PRR}}=27 \mathrm{~ms}, p=0.0026\right)$. In contrast, in the $S$ condition (Fig. $3 B$ ) there was a trend for earlier motor tuning in PMd than PRR in pro-reaches. The LD was close to significant when we selected the neurons according to the standard criterion for spatial tuning, as defined in Materials and Methods $\left(\mathrm{LD}_{\mathrm{PRR} \_\mathrm{PMd}}=19 \mathrm{~ms}, p=0.057\right)$ (Fig. $3 B$ ). It was significant $\left(\mathrm{LD}_{\mathrm{PRR} \longrightarrow \mathrm{PMd}}=25 \mathrm{~ms}, p=0.026\right)$ when neurons were selected according to a stricter tuning criterion, which we did as a control (supplemental Fig. S1, available at www.jneurosci.org as supplemental material). There was no significant LD in antireaches in the $S$ condition $\left(\mathrm{LD}_{\mathrm{PRR}-\mathrm{PMd}}=3 \mathrm{~ms}, p>0.05\right)$ for either selection criterion (Fig. 3B; supplemental Fig. S1, available at www.jneurosci.org as supplemental material). Note that the higher baseline level in the S anti-trials can be explained by the spatial tuning during the $S$ condition memory period, as will be discussed below.

The results of the RS, N, and R conditions, which showed LDs only for anti-goals but not for pro-goals, suggest that the frontoparietal LDs could reflect the process of spatial remapping required in anti- but not pro-reaches. The LDs that we found in the $S$ condition during pro- but not anti-reaches seem to be at odds with this remapping hypothesis. In the following, we will demonstrate that the seemingly counterintuitive results of the $\mathrm{S}$ condition are consistent with the idea that spatial remapping is the relevant factor for inducing motor-goal LDs between PRR and PMd. For this, we analyzed the neural encoding and putative cognitive strategy of the monkeys in the memory periods of the $\mathrm{R}$ and $\mathrm{S}$ conditions, first with respect to the context encoding, and second-and more importantly-with respect to the spatial encoding.

Figure 4 shows the comparative dynamics of context tuning in PMd and PRR. The context was represented earlier and more prevalently in PMd than PRR. Toward the end of the R condition memory period, the fraction of rule-tuned cells was only slightly higher in PMd than PRR, consistent with earlier reports (Gail et al., 2009). This finding does not imply any specific effect on the relative time courses of motor-goal encoding in PMd versus PRR after the spatial go-cue at the end of the R condition memory period.

The situation is different in the $\mathrm{S}$ condition. The $\mathrm{S}$ condition leaves some ambiguity as to what the animals memorize during the memory period of these trials: (1) a memory of the spatial precue; (2) the two potential motor goals (pro/anti), which are
A PMd $(\mathrm{N}=53)$
Rule \& Spatial (RS)

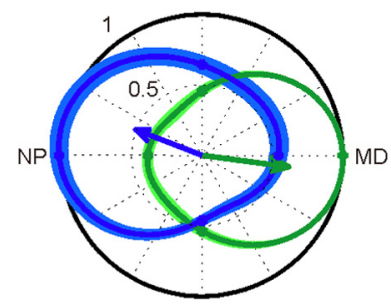

pro

$B \operatorname{PRR}(\mathrm{N}=54)$

Rule \& Spatial (RS)

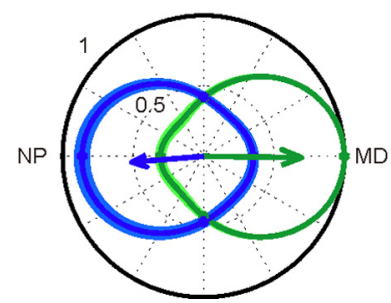

Spatial only (S)

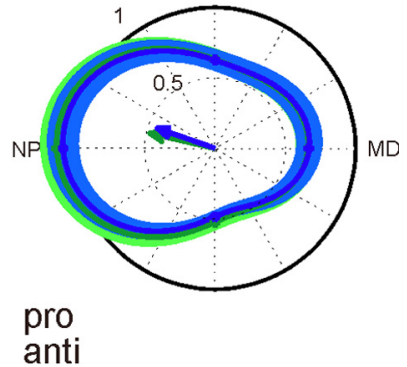

Spatial only (S)

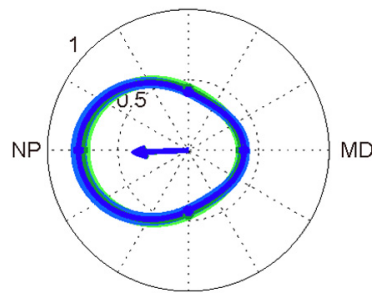

Figure 5. Predominant spatial tuning preference for the anti-goal in the memory period of the $S$ condition. $\boldsymbol{A}, \boldsymbol{B}$, The normalized population tuning in pro-trials (green) and anti-trials (blue) is shown relative to the preferred spatial cue position in PMd $(\boldsymbol{A})$ and PRR $(\boldsymbol{B})$. Tuning curves of each neuron were aligned such that their MD in pro-reaches corresponded to $0^{\circ}$ before averaging across neurons. The population tuning in the RS memory periods (left) confirms the typical expected motor-goal tuning in PMd and PRR. The population tuning in the $S$ conditions (right) indicates preference for the anti-goal. Note that the memory period of the $S$ condition precedes the go-cue. Since the transformation rule (pro or anti) could not be known by the monkeys, the tuning curves for pro- and anti-trials are practically identical. Only motor-goal neurons were used for this analysis (see Materials and Methods).

associated with each spatial cue as long as the context is not known; and (3) a preliminary default movement plan to either the pro- or anti-goal. If the monkeys memorized the spatial precue or by default planned a pro-reach during the memory period of the $S$ condition, then a spatial remapping would have been required after the contextual go-cue during anti- but not proreaches, as was the case in the other cueing conditions. If, on the other hand, the monkeys by default planned an anti-reach during the memory period of the $S$ condition, then a spatial remapping would have been required after the contextual go-cue during probut not anti-reaches. To test whether such a need for spatial remapping during $\mathrm{S}$ condition pro-reaches can explain the observed LDs in this condition, we analyzed the population tuning during the memory period of the $\mathrm{S}$ condition before the contextual go-cue was shown. Only the population of motor-goal neurons (see Materials and Methods) was used for this analysis, because for other neurons we could not expect a sustained response during the memory period. In PMd, two of the motorgoal neurons dropped out of the analysis because of a lack of activity at the end of the $S$ condition memory period (spike density at $\mathrm{MD},<1 \mathrm{~Hz})$.

Importantly, and against intuition, in both monkeys neurons in PMd and PRR in this experiment almost exclusively encoded the anti-goal during the memory period of the $\mathrm{S}$ condition. Figure 5 shows the population tuning of motor-goal neurons for PMd and PRR in the last $200 \mathrm{~ms}$ of the memory period in the RS 
and the $\mathrm{S}$ conditions (the baseline period for the recruitment curves). The time window was chosen to analyze the activity in the $\mathrm{S}$ condition immediately before the contextual information was given to the monkey. The tuning curves of all motor-goal neurons were aligned and normalized with respect to their maximal response (plotted at $0^{\circ}$ ), which was calculated as the average spike density in the $200 \mathrm{~ms}$ across pro-trials in the RS condition. The motor-goal tuning in the RS condition was taken as a reference to be able to differentiate in the $\mathrm{S}$ condition between tuning to the pro- and to the anti-goal. Neurons in PMd and PRR in the RS condition (Fig. $5 A, B$, left column) on average show the typical opposing tuning curves between pro- and anti-trials, reflecting their motor-goal encoding (Fig. 1C). The right columns of Figure $5, A$ and $B$, show that the same neurons in the memory period of the $\mathrm{S}$ condition are spatially tuned toward the anti-reach direction. We interpret this tuning as a preliminary default movement plan of both monkeys to the anti-goal. It suggests that after the contextual go-cue in the $\mathrm{S}$ condition a remapping is indeed required in pro- but not in anti-reaches, consistent with the neural LD data above.

Figure 6, $A$ and $B$, summarizes the latency results for PMd and $\mathrm{PRR}$ in all conditions. There was a significant difference between PMd and PRR motor-related latencies in only those conditions where a spatial remapping was required (RS-anti, N-anti, R-anti, $\mathrm{S}$-pro). There was no LD in conditions without remapping (RSpro, N-pro, R-pro, S-anti).

Motor planning versus motor feedback in reaction-time trials Sensorimotor areas, especially in the posterior parietal cortex, are activated by movement-induced somatosensory or visual feedback (Sakata et al., 1973; Mountcastle et al., 1975; Kalaska et al., 1983; Colby and Duhamel, 1991), not only by sensory cues or motor planning activity. The motor-goal LDs between PMd and PRR in the three RT conditions (N, R, and S) could be confounded by such motor feedback, including corollary discharge signals, if, for example, PRR was more strongly driven by movement-induced feedback signals, whereas PMd was more strongly driven by motor planning signals. To test whether during movement initiation in RT conditions the LDs between PMd and PRR were related to motor planning rather than motor feedback, we split our data set into motor-goal neurons (N_PMd/ $\mathrm{PRR}=65 / 75)$ and a complementary group of perimovement neurons (N_PMd/PRR $=127 / 183)$. Motor-goal neurons were characterized by motor-related tuning during the memory period of the RS condition and perimovement neurons by motorrelated tuning in the movement period, but not during the memory period (see Materials and Methods). Figure $6 \mathrm{C}$ shows the LDs between PRR and PMd averaged across all RT conditions without spatial remapping (N-pro, R-pro, S-anti) and with spatial remapping ( $\mathrm{N}$-anti, $\mathrm{R}$-anti, S-pro). The average LDs were computed separately for the population of all neurons, the population of motor-goal neurons, and the population of perimovement neurons. In the conditions without remapping, there were no significant LDs for either group. In the conditions with remapping, there were significant LDs between PMd and PRR for the population of all neurons ( $23 \mathrm{~ms}, p=0.017)$ and the motorgoal neurons ( $21 \mathrm{~ms}, p=0.03$ ). For the perimovement neurons, there was only a nonsignificant trend $(21 \mathrm{~ms}, p=0.084)$, despite the fact that the number of eligible neurons in this category was higher than that for the motor-goal neurons. The absolute latencies for the motor-goal tuning were shorter in the group of motor-goal neurons than the perimovement neurons (average across all three RT conditions and both contexts: $\Delta=30 \mathrm{~ms}$;
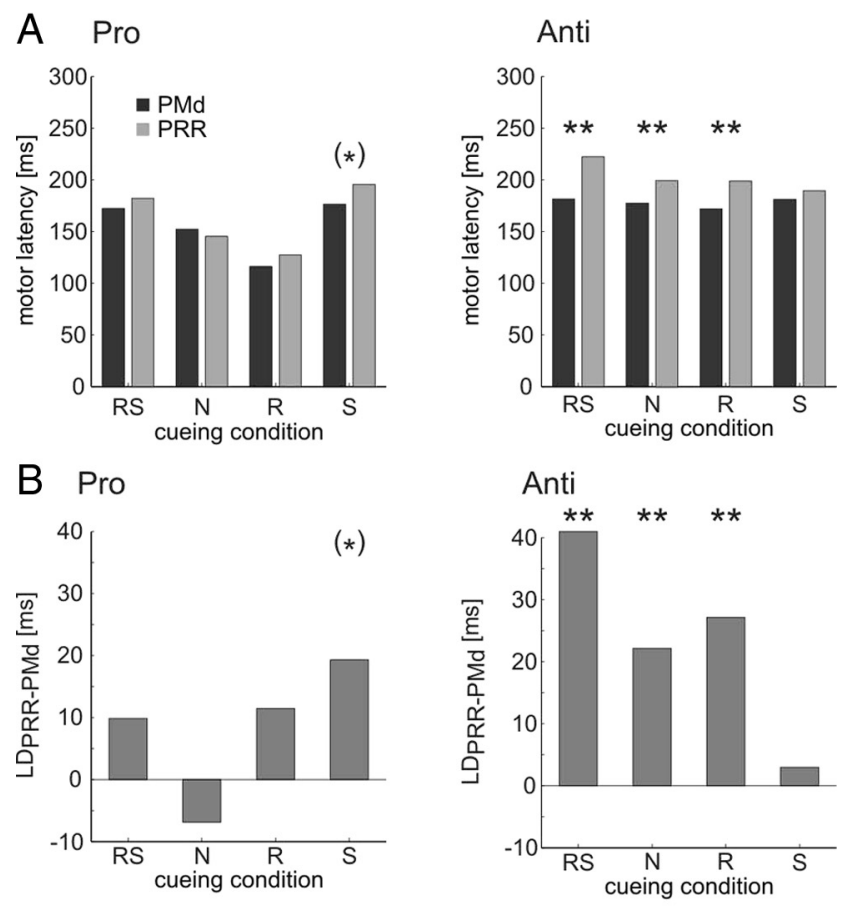

\section{No Remapping}

\section{Remapping}
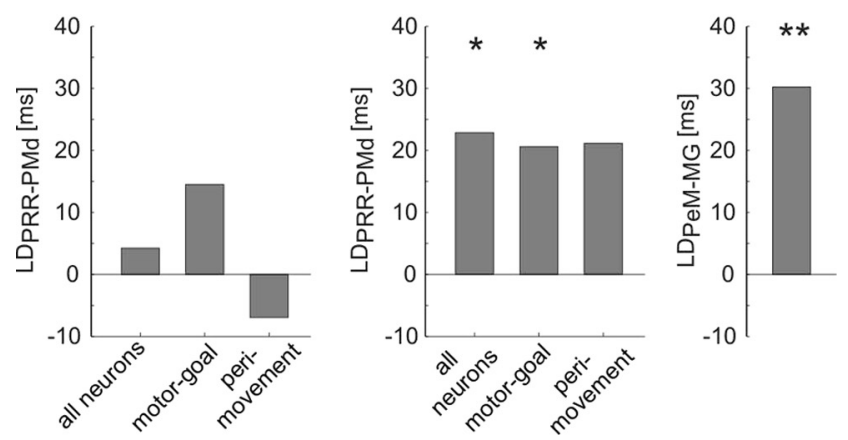

Figure 6. Summary of frontoparietal latencies and split analysis for different neuron populations. $\boldsymbol{A}, \boldsymbol{B}$, Summary of average absolute latencies $(\boldsymbol{A})$ and $\operatorname{LD}(\boldsymbol{B})$ between PRR and PMd for motor-related tuning for pro-trials (left) and anti-trials (right) for all cueing conditions (RS, N, R,S). C, Comparison of remapping conditions with other conditions. LDs between PRR and PMd (PRR - PMd) averaged across all $\mathrm{RT}$ conditions with no required remapping ( $\mathrm{N}$-pro, $\mathrm{R}$-pro, $\mathrm{S}$-anti) and with required remapping (N-anti, R-anti, S-pro) for either all neurons or separately for motor-goal (MG) and perimovement (PeM) neurons. Asterisks indicate the level of significance $\left({ }^{*} p<0.05 ;{ }^{* *} p<0.01\right.$, randomization test). See Results and supplemental material (available at www.jneurosci.org as supplemental material) for the significance of the S-pro condition. Only remapping conditions induce significant frontoparietal LDs. These differences are visible mainly in motor-goal neurons, less so in perimovement neurons. $\boldsymbol{D}$, Average tuning latencies are smaller in motor-goal compared with perimovement neurons. The bar shows LDs (PeM - MG) across all RT conditions and both cortical areas.

$p=0.00049$ ) (Fig. 6D). This difference in absolute latency confirmed that the splitting of the two groups of neurons according to their tuning properties in the RS condition memory period was meaningful with respect to the differentiation between motorgoal and motor-feedback latencies.

\section{Effects of remapping on motor-goal latencies within PMd and PRR}

We also compared motor-goal latencies between pro- and antireaches within each cortical area, rather than latencies between cortical areas (Fig. 7). Note that these are the same data as in the above analyses, just rearranged for different statistical comparisons. Pro-goal tuning emerged earlier than anti-goal tuning in 


\section{PMd}

A
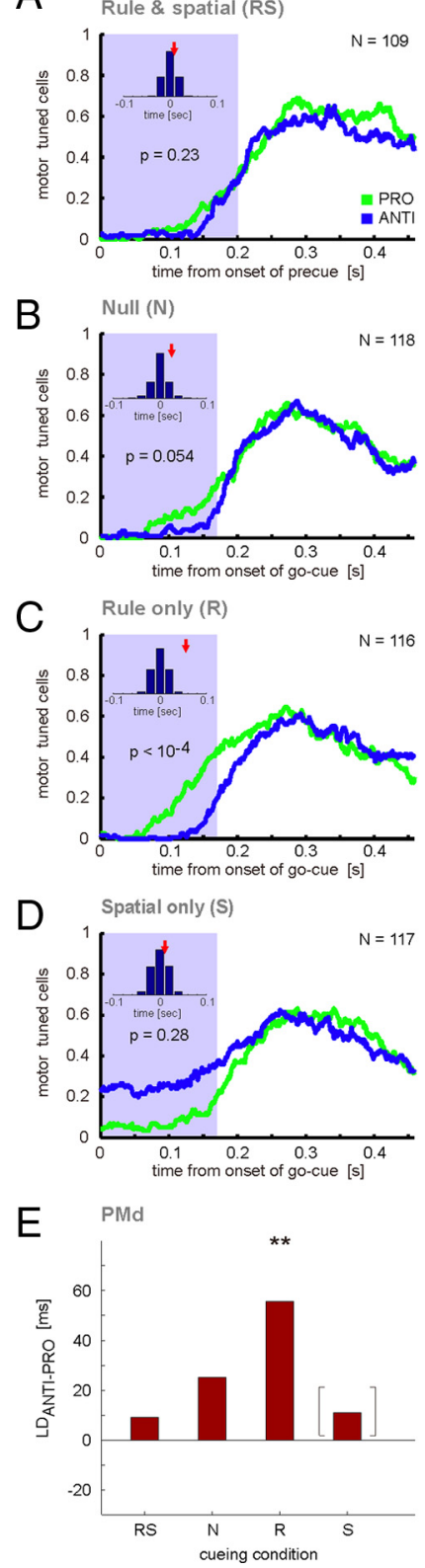

Figure 7. Comparison of motor-tuning latencies between pro- and anti-trials. $A-D$, Recruitment curves for all four cueing conditions in pro-trials (green) and anti-trials (blue), separately for PMd (left) and PRR (right). Other conventions are as in Figures 2 and 3 . Note that the data are the same as in Figures 2 and 3 , but are grouped differently to emphasize and quantify the differences between pro- and anti-trials rather than PMd and PRR. $\boldsymbol{E}$, Summary of LDs between pro- and anti-trials (anti - pro). Brackets for the $S$ condition indicate that the conclusiveness of LDs is questionable because of the different baseline levels in this condition.

PRR whenever the motor-goal decision was immediately preceded by a spatial cue: pro-anti-LDs were significant after the precue in the RS condition $\left(\mathrm{LD}_{\mathrm{A}-\mathrm{P}}=40 \mathrm{~ms}, p=0.0021\right)$, after the go-cue in the $\mathrm{N}$ condition $\left(\mathrm{LD}_{\mathrm{A}-\mathrm{P}}=54 \mathrm{~ms}, p=0\right)$, and after the go-cue in the $\mathrm{R}$ condition $\left(\mathrm{LD}_{\mathrm{A}-\mathrm{P}}=71 \mathrm{~ms}, p=0\right)$. There was no pro-anti-LD in response to the go-cue in the $\mathrm{S}$ condition $(p=$ 0.32). In PMd, we found faster representation of pro-goals compared with anti-goals only in response to the go-cue in the $\mathrm{R}$ condition $\left(\mathrm{LD}_{\mathrm{P}-\mathrm{A}}=56 \mathrm{~ms}, p<10^{-4}\right)$. In $\mathrm{PMd}$, none of the other conditions showed a significant difference between proand anti-goals (RS: $p=0.27 ; \mathrm{N}: p=0.054 ; \mathrm{S}: p=0.42$ ). Note that for the $\mathrm{S}$ condition in either area the LD analysis between proand anti-trials is confounded by the default anti-tuning in the preceding memory period, which makes interpretation in this case difficult.

\section{Motor-goal latencies and behavioral reaction times}

If motor-goal latencies in PMd and PRR reflect decisions on arm movements, a correlation between motor-goal latencies and RTs should be expected. Figure $8 \mathrm{~A}$ shows the median RTs in proreaches (light gray) and anti-reaches (dark gray) for the different cueing conditions averaged across all recording sessions $(N=$ 176). The RTs for pro-reaches were $240 \pm 2 \mathrm{~ms}$ (RS), $227 \pm 2 \mathrm{~ms}$ $(\mathrm{N}), 221 \pm 2 \mathrm{~ms}(\mathrm{R})$, and $240 \pm 2 \mathrm{~ms}(\mathrm{~S})$. The RTs in anti-reaches were $246 \pm 2(\mathrm{RS}), 242 \pm 2 \mathrm{~ms}(\mathrm{~N}), 245 \pm 2 \mathrm{~ms}(\mathrm{R})$, and $244 \pm$ $2 \mathrm{~ms}(\mathrm{~S})$. The RT differences between pro- and anti-reaches were significant in each cueing condition (RS: $6 \pm 1 \mathrm{~ms}, p<10^{-6} ; \mathrm{N}$ : $15 \pm 1 \mathrm{~ms}, p<10^{-6}$; R: $24 \pm 1 \mathrm{~ms}, p<10^{-6}$; $\mathrm{s}: 4 \pm 1 \mathrm{~ms} ; p=4.6$ $10^{-5}$; data not shown). The RT differences between pro- and anti-reaches in the RS condition cannot be explained by motorgoal latencies, since motor-goal tuning in these conditions was already present during the memory period. To compensate for RT differences between pro- and anti-trials for the nonspecific effects of SR congruency observed in the RS condition, we also computed RT differences that were corrected for this nonspecific effect by subtracting the RT difference in the RS condition from the original RT differences (Fig. $8 B$ ). The corrected RT differences were $9 \pm 1 \mathrm{~ms}$ ( $\mathrm{N}$ condition, $\left.p<10^{-6}\right), 18 \pm 1 \mathrm{~ms}(\mathrm{R}, p<$ $\left.10^{-6}\right)$, and $-2 \pm 1 \mathrm{~ms}(\mathrm{~S}, p=0.015)$.

We tested whether the neuronal motor-goal latencies better correlated with the time of the cue presentation or with the behavioral response onset (reaction time). The top left panel of Figure $8 C$ shows as an example the slopes of the linear regression between single-trial RTs and neuronal onset latencies (relative to cue presentation; see Materials and Methods) for the motor-goal neurons in PRR. In a complementary manner, the slopes of the regression between (negative) single-trial RTs and neuronal lead times (onset of neuronal response relative to movement onset) were also analyzed (Fig. $8 C$, top right). Note that these two ways of quantifying the relationship between neural latencies and behavioral responses are not mutually independent, but help to illustrate the results. For the regression analysis, we only included trials of the RT conditions ( N, R, and S) from neurons that did not show cue responses (see Materials and Methods). A slope close to 1 in the comparison between RTs and neuronal onset latencies (top left) indicates that the onset of activity in PRR motor-goal neurons was correlated to the movement onset. A slope close to 1 in the comparison between RTs and neuronal lead times (top right) would have indicated that the onset of activity was related to the cue onset. Overlap of the regression lines with the upper gray-shaded area indicates neural latencies larger than the RT, which only occurred in a small fraction of the motor-goal neurons. The pattern of slopes showed better correlation of the neural latencies with the movement onset compared with the cue onset for PRR motor-goal neurons, and also for perimovement neurons (Fig. $8 C$, bottom right). For motor-goal neurons in PRR, the median slope for relatedness to RT was 0.76 , and for relatedness to cue onset 0.14 ( $p=0.00026$, rank sum test). For perimovement neurons in PRR, the median slope for RT was 0.62 , and for cue onset 0.20 ( $p<10^{-5}$, rank sum test). In PMd, we could not find a significant bias in either neural population. For motor-goal neurons in PMd, the median slope for RT was 0.60, and for cue onset 0.25 ( $p>0.05)$. For perimovement neurons, the median slope for RT was 0.47 and for cue onset 0.39 ( $p>$ 
0.05). The distribution of slopes for both neural populations was unimodal and did not suggest that the moderate correlation with either cue onset or RT is the result of an averaging effect across an inhomogeneous distribution of neurons (data not shown). In summary, in PRR neural motor-goal latencies were best correlated with the time of the behavioral response; in PMd, there was no bias, which would indicate stronger correlation of the neuronal latencies with either the cue onset or the reaction time.

\section{Statistical controls}

Latency measures depend on methodological procedures. For example, any statistical significance level (here, $\alpha=5 \%$ ) for motor-related tuning (significant spatial tuning in pro- and anti-reaches with opposite tuning vectors) marks an arbitrary threshold criterion. To avoid confounds caused by potential differences of the effect size in PMd and PRR, we performed our analysis using different threshold criteria for spatial tuning (supplemental Fig. S1, available at www. jneurosci.org as supplemental material). Also, we balanced the number of neurons between PMd and PRR by random subsampling (supplemental Fig. S2, available at www.jneurosci.org as supplemental material). Finally, we used alternative latency measures based on cumulative sums, as were used in previous studies from other groups (supplemental Fig. S3, available at www.jneurosci.org as supplemental material). None of the control measurements or alternative methods led to conclusions different from those presented in the main text.

\section{Discussion}

We compared motor-goal latencies in PMd and PRR during a nonstandard visuomotor association task. Neurons in PMd were tuned earlier for the pending motor goal than neurons in PRR, whenever the task required spatial visuomotor remapping. Frontoparietal LDs existed for motor goals during movement planning, independent of movement execution. We interpret our findings in support of the hypothesis that spatial reorganization of network activity in PRR causes frontoparietal LDs. We suggest that the initiation of the remapping process in PRR is contingent on frontoparietal projections from PMd.

\section{Shorter motor-goal latencies in PMd than PRR in remapping conditions}

We expected the relative timing of motor-goal decision processes between reach planning areas to change with precueing, as speculated previously (Kalaska and Crammond, 1992; Cisek, 2006; 2007). Against our expectation, PMd-PRR latency differences depended on the required visuospatial mapping instead. Variation of the precue did not change this basic dependency. Our results showed earlier motor-goal tuning in PMd than PRR whenever the reach goal had to be spatially remapped from the position of the preceding incongruent spatial cue (anti-trials in $\mathrm{RS}, \mathrm{N}$, and R conditions) or the incongruent preliminary movement plan (pro-trials in $\mathrm{S}$ condition).
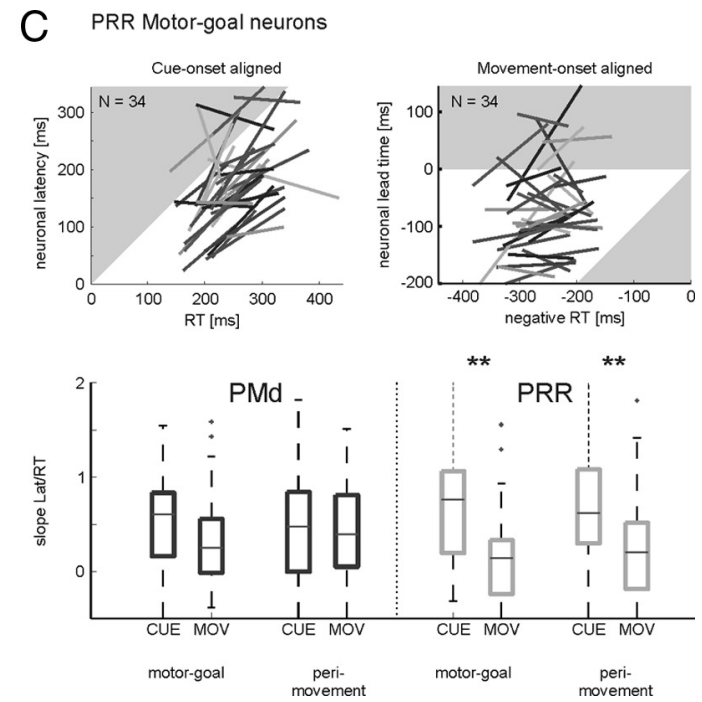
movement alignment (MOV) in both areas separately for motor-goal and perimovement neurons. In both groups of neurons, neural latencies in PRR but not PMd were correlated with behavioral response latencies.

Different reasons could account for the dependency of frontoparietal LDs on spatial remapping. It was previously suggested that frontoparietal interactions and corresponding LDs are the consequences of a decision-making process (Pesaran et al., 2008). In line with this view, one could emphasize the inherent difference in cognitive strategy required to perform either a pro-reach or an anti-reach task. To be consistent with our data, we would have to assume that all conditions that include a remapping require an active decision, whereas conditions without remapping do not. The function of such a decision could be the active updating of the preliminary motor goal, which was induced by the spatial cue or preliminary movement planning.

Alternatively, PRR motor-goal representations could depend on frontoparietal projections from PMd in any case, while becoming visible as interareal LDs only under specific circumstances. LDs of several tens of milliseconds, as seen here and also in previous studies (Riehle, 1991; Kalaska and Crammond, 1992; Johnson et al., 1996; Pesaran et al., 2008), seem rather long to be attributable to transmission delays between the monosynaptically connected areas PMd and PRR (Pandya and Kuypers, 1969; Jones and Powell, 1970; Kurata, 1991; Johnson et al., 1996; Caminiti et al., 1999; Marconi et al., 2001; Tanné-Gariépy et al., 2002; BattagliaMayer et al., 2003). Instead, we prefer to attribute the observed large frontoparietal LDs to the dynamic reorganization of network activity, which is required in visuospatially organized areas like PRR in the case of spatial remapping. According to this view, the dynamic reorganization is only started in PRR, once the motor goal has been selected in PMd. The dependency of PRR on PMd output would only become detectable as a frontoparietal LD in cases of spatially incongruent mapping, when an isolated spatial motor-goal representation is not directly induced by the spatial cue, but has to build up first. We predict that tasks with 
nonspatial symbolic cueing could also induce frontoparietal LDs, similar to tasks with a spatially balanced distribution of cues (visual search task, Pesaran et al., 2008). What is common to all three tasks is the need for the buildup of a spatial goal representation in PRR that is not induced directly by a spatial cue. Spatially congruent mapping conditions, on the other hand, do not require dynamic remapping or buildup. The seamless and immediate transition from cue- to motor-goal-related tuning, even if its "initiation" in PRR might depend on PMd output, would render frontoparietal LDs invisible in this case.

The latter interpretation fits our and previous data, and explains long frontoparietal motor-goal LDs independently of interareal transmission delays. It is parsimonious since it requires neither an assumption that PMd-to-PRR projections become active only during decision tasks nor that remapping trials involve a decision process, whereas other trials do not. Note that we cannot exclude the alternative, but similar, possibility that both areas, PMd and PRR, receive a motor-goal decision signal from an independent third area, and that PRR in the case of remapping needs longer than PMd to converge onto a new dynamic state to represent the spatial motor goal.

\section{Latency differences between PMd and PRR for motor planning activity}

Previous physiological studies suggested that frontoparietal LDs between reach-related areas in premotor and parietal cortices exist for perimovement activity immediately preceding or accompanying motor execution (Bioulac and Lamarre, 1979; Georgopoulos et al., 1982; Kalaska et al., 1983; Riehle and Requin, 1989; Riehle, 1991; Johnson et al., 1996), in line with a previously stated corollary discharge hypothesis (Mountcastle et al., 1975; Bioulac and Lamarre, 1979; Kalaska et al., 1983; Seal and Commenges, 1985; Johnson et al., 1996). A previous study (Pesaran et al., 2008) showed that latency differences between PMd and PRR also exist for motor-goal representations during movement planning, i.e., independent of movement execution, whereas another study did not show such differences (Johnson et al., 1996). The fact that frontoparietal LDs depend on the type of task rather than the movement execution can explain these different results. We found shorter latencies in PMd than PRR for motor-goal activity in response to a preparatory stimulus (RS condition) and in response to a go-instruction ( $\mathrm{N}, \mathrm{R}$, and $\mathrm{S}$ conditions). Even in the RT conditions, we could attribute the frontoparietal LDs to differences in the latencies of motor-goal signals, rather than motor feedback signals. Our findings contradict a corollary discharge hypothesis in its strict sense (see alternative suggestion below), in which PMd-PRR latency differences would be the consequence of a frontoparietal efference copy signal, i.e., would be dependent on explicit motor commands (Kalaska et al., 1983; Johnson et al., 1996).

\section{Motor-goal latencies in relation to behavioral reaction times}

To discuss the neural dynamics of sensorimotor integration in the context of behavioral performance, it is important to distinguish between general neural response onset latencies (Kalaska et al., 1983; Riehle, 1991; Kalaska and Crammond, 1992; Johnson et al., 1996; Pesaran et al., 2008) and latencies of neural activities that are more specifically related to the cue, the motor-goal decision, or motor execution.

First, with the anti-reach task design we separated cue- from motor-related tuning (Crammond and Kalaska, 1994; Zhang et al., 1997; Gail and Andersen, 2006). Neurons in PMd (Crammond and Kalaska, 1994) and PRR (Gail and Andersen, 2006) can dynamically switch from cue- to motor-related encoding, whereas others are motor related as soon as they become active. Population analysis of neural onset latencies including all neurons would lead to confounded motor latencies. Especially, frontoparietal LDs could be obscured because of the simultaneity of the fastest responding neurons (Johnson et al., 1996), which respond to the cue. We avoided this confound by analyzing neurons without cue-related responses.

Second, using different partial precueing conditions (Lecas et al., 1986; Riehle and Requin, 1989; Riehle, 1991; Hoshi and Tanji, 2000; Nakayama et al., 2008), we additionally separated motor-goal latencies related to motor planning (Riehle, 1991; Johnson et al., 1996) from latencies of perimovement activity related to motor-execution (Riehle and Requin, 1989; Crammond and Kalaska, 2000). Perimovement activities could comprise movement-induced corollary discharge, somatosensory, or visual signals, especially in parietal areas (Sakata et al., 1973; Mountcastle et al., 1975; Kalaska et al., 1983; Colby and Duhamel, 1991; Nakayama et al., 2008). Average response latencies from mixed motor-goal and perimovement activity (Kalaska et al., 1983; Riehle and Requin, 1989; Johnson et al., 1996; Pesaran et al., 2008) would overestimate motor-goal latencies in areas with strong motor-feedback signals. We avoided this confound by differentiating motor-goal from perimovement neurons.

The trial-by-trial motor-goal latencies in PRR were better correlated with manual RTs than those of PMd. This could mean that manual responses are triggered only once the motor-goal decision (which becomes visible in PMd before PRR) leads to the required (and slower) evolution of the proper motor-goal representations in PRR.

\section{Frontoparietal projections for prospective forward model estimates?}

Parietal cortex function is important for proper online correction of movements (Desmurget et al., 1999) and is suspected to use forward model predictions for this purpose (Wolpert et al., 1998; Desmurget et al., 1999; Mulliken et al., 2008; Shadmehr and Krakauer, 2008). We suggest that such a forward estimate is generated not only during movements, but prospectively, before a motor command is issued, as an integral part of the planning process. In visuomotor tasks, movement planning then would equal the process of analyzing the difference between the current sensory state and the prospective sensory state, which is predicted to result from the currently imagined movement in the future, once it is executed (Salinas, 2004; Shadmehr and Wise, 2005). This view is consistent with our data and previous studies, which showed activation of PPC during motor imagery (Decety et al., 1994; Stephan et al., 1995; Sirigu et al., 1996; Gerardin et al., 2000; Naito et al., 2002), and the fact that parietal electrical stimulation can trigger subjective movement intentions without actual movements being performed (Desmurget et al., 2009).

\section{References}

Battaglia-Mayer A, Caminiti R, Lacquaniti F, Zago M (2003) Multiple levels of representation of reaching in the parieto-frontal network. Cereb Cortex 13:1009-1022.

Bioulac B, Lamarre Y (1979) Activity of postcentral cortical neurons of the monkey during conditioned movements of a deafferented limb. Brain Res 172:427-437.

Boussaoud D, Wise SP (1993) Primate frontal-cortex-effects of stimulus and movement. Exp Brain Res 95:28-40.

Brozović M, Gail A, Andersen RA (2007) Gain mechanisms for contextually guided visuomotor transformations. J Neurosci 27:10588-10596.

Buneo CA, Andersen RA (2006) The posterior parietal cortex: sensorimotor interface for the planning and online control of visually guided movements. Neuropsychologia 44:2594-2606.

Caminiti R, Genovesio A, Marconi B, Mayer AB, Onorati P, Ferraina S, 
Mitsuda T, Giannetti S, Squatrito S, Maioli MG, Molinari M (1999) Early coding of reaching: frontal and parietal association connections of parieto-occipital cortex. Eur J Neurosci 11:3339-3345.

Cisek P (2006) Integrated neural processes for defining potential actions and deciding between them: a computational model. J Neurosci 26:97619770.

Cisek P (2007) Cortical mechanisms of action selection: the affordance competition hypothesis. Philos Trans R Soc Lond B Biol Sci 362: 1585-1599.

Colby CL, Duhamel JR (1991) Heterogeneity of extrastriate visual areas and multiple parietal areas in the macaque monkey. Neuropsychologia 29:517-537.

Crammond DJ, Kalaska JF (1994) Modulation of preparatory neuronal activity in dorsal premotor cortex due to stimulus-response compatibility. J Neurophysiol 71:1281-1284.

Crammond DJ, Kalaska JF (2000) Prior information in motor and premotor cortex: activity during the delay period and effect on pre-movement activity. J Neurophysiol 84:986-1005.

Decety J, Perani D, Jeannerod M, Bettinardi V, Tadary B, Woods R, Mazziotta JC, Fazio F (1994) Mapping motor representations with positron emission tomography. Nature 371:600-602.

Desmurget M, Epstein CM, Turner RS, Prablanc C, Alexander GE, Grafton ST (1999) Role of the posterior parietal cortex in updating reaching movements to a visual target. Nat Neurosci 2:563-567.

Desmurget M, Reilly KT, Richard N, Szathmari A, Mottolese C, Sirigu A (2009) Movement intention after parietal cortex stimulation in humans. Science 324:811-813.

di Pellegrino G, Wise SP (1993) Visuospatial versus visuomotor activity in the premotor and prefrontal cortex of a primate. J Neurosci 13:12271243.

Eliassen JC, Souza T, Sanes JN (2003) Experience-dependent activation patterns in human brain during visual-motor associative learning. J Neurosci 23:10540-10547.

Gail A, Andersen RA (2006) Neural dynamics in monkey parietal reach region reflect context-specific sensorimotor transformations. J Neurosci 26:9376-9384.

Gail A, Klaes C, Westendorff S (2009) Implementation of spatial transformation rules for goal-directed reaching via gain modulation in monkey parietal and premotor cortex. J Neurosci 29:9490-9499.

Georgopoulos AP, Kalaska JF, Caminiti R, Massey JT (1982) On the relations between the direction of two-dimensional arm movements and cell discharge in primate motor cortex. J Neurosci 2:1527-1537.

Gerardin E, Sirigu A, Lehéricy S, Poline JB, Gaymard B, Marsault C, Agid Y, Le Bihan D (2000) Partially overlapping neural networks for real and imagined hand movements. Cereb Cortex 10:1093-1104.

Hoshi E, Tanji J (2000) Integration of target and body-part information in the premotor cortex when planning action. Nature 408:466-470.

Johnson PB, Ferraina S, Bianchi L, Caminiti R (1996) Cortical networks for visual reaching: physiological and anatomical organization of frontal and parietal lobe arm regions. Cereb Cortex 6:102-119.

Jones EG, Powell TP (1970) An anatomical study of converging sensory pathways within the cerebral cortex of the monkey. Brain 93:793-820.

Kalaska JF (1996) Parietal cortex area 5 and visuomotor behavior. Can J Physiol Pharmacol 74:483-498.

Kalaska JF, Crammond DJ (1992) Cerebral cortical mechanisms of reaching movements. Science 255:1517-1523.

Kalaska JF, Caminiti R, Georgopoulos AP (1983) Cortical mechanisms related to the direction of two-dimensional arm movements-relations in parietal area 5 and comparison with motor cortex. Exp Brain Res 51: 247-260.

Kalaska JF, Scott SH, Cisek P, Sergio LE (1997) Cortical control of reaching movements. Curr Opin Neurobiol 7:849-859.

Kurata K (1991) Corticocortical inputs to the dorsal and ventral aspects of the premotor cortex of macaque monkeys. Neurosci Res 12:263-280.

Lecas JC, Requin J, Anger C, Vitton N (1986) Changes in neuronal activity of the monkey precentral cortex during preparation for movement. J Neurophysiol 56:1680-1702.

Marconi B, Genovesio A, Battaglia-Mayer A, Ferraina S, Squatrito S, Molinari M, Lacquaniti F, Caminiti R (2001) Eye-hand coordination during reaching. I. Anatomical relationships between parietal and frontal cortex. Cereb Cortex 11:513-527.

Mitz AR, Godschalk M, Wise SP (1991) Learning-dependent neuronal ac- tivity in the premotor cortex: activity during the acquisition of conditional motor associations. J Neurosci 11:1855-1872.

Monosov IE, Trageser JC, Thompson KG (2008) Measurements of simultaneously recorded spiking activity and local field potentials suggest that spatial selection emerges in the frontal eye field. Neuron 57:614-625.

Mountcastle VB, Lynch JC, Georgopoulos A, Sakata H, Acuna C (1975) Posterior parietal association cortex of the monkey: command functions for operations within extrapersonal space. J Neurophysiol 38:871-908.

Mulliken GH, Musallam S, Andersen RA (2008) Decoding trajectories from posterior parietal cortex ensembles. J Neurosci 28:12913-12926.

Naito E, Kochiyama T, Kitada R, Nakamura S, Matsumura M, Yonekura Y, Sadato N (2002) Internally simulated movement sensations during motor imagery activate cortical motor areas and the cerebellum. J Neurosci 22:3683-3691.

Nakayama Y, Yamagata T, Tanji J, Hoshi E (2008) Transformation of a virtual action plan into a motor plan in the premotor cortex. J Neurosci 28:10287-10297.

Pandya DN, Kuypers HG (1969) Cortico-cortical connections in the rhesus monkey. Brain Res 13:13-36.

Passingham RE, Toni I, Rushworth MF (2000) Specialisation within the prefrontal cortex: the ventral prefrontal cortex and associative learning. Exp Brain Res 133:103-113.

Pesaran B, Nelson MJ, Andersen RA (2008) Free choice activates a decision circuit between frontal and parietal cortex. Nature 453:406-409.

Riehle A (1991) Visually induced signal-locked neuronal activity changes in precentral motor areas of the monkey: hierarchical progression of signal processing. Brain Res 540:131-137.

Riehle A, Requin J (1989) Monkey primary motor and premotor cortex: single-cell activity related to prior information about direction and extent of an intended movement. J Neurophysiol 61:534-549.

Sakata H, Takaoka Y, Kawarasaki A, Shibutani H (1973) Somatosensory properties of neurons in the superior parietal cortex (area 5) of the rhesus monkey. Brain Res 64:85-102.

Salinas E (2004) Fast remapping of sensory stimuli onto motor actions on the basis of contextual modulation. J Neurosci 24:1113-1118.

Seal J, Commenges D (1985) A quantitative analysis of stimulus- and movement-related responses in the posterior parietal cortex of the monkey. Exp Brain Res 58:144-153.

Shadmehr R, Krakauer JW (2008) A computational neuroanatomy for motor control. Exp Brain Res 185:359-381.

Shadmehr R, Wise SP (2005) The computational neurobiology of reaching and pointing: a foundation for motor learning. Cambridge, MA: MIT.

Sirigu A, Duhamel JR, Cohen L, Pillon B, Dubois B, Agid Y (1996) The mental representation of hand movements after parietal cortex damage. Science 273:1564-1568.

Stephan KM, Fink GR, Passingham RE, Silbersweig D, Ceballos-Baumann AO, Frith CD, Frackowiak RS (1995) Functional anatomy of the mental representation of upper extremity movements in healthy subjects. J Neurophysiol 73:373-386.

Stoet G, Snyder LH (2004) Single neurons in posterior parietal cortex of monkeys encode cognitive set. Neuron 42:1003-1012.

Tanné-Gariépy J, Rouiller EM, Boussaoud D (2002) Parietal inputs to dorsal versus ventral premotor areas in the macaque monkey: evidence for largely segregated visuomotor pathways. Exp Brain Res 145:91-103.

Thompson KG, Hanes DP, Bichot NP, Schall JD (1996) Perceptual and motor processing stages identified in the activity of macaque frontal eye field neurons during visual search. J Neurophysiol 76:4040-4055.

Wallis JD, Miller EK (2003) from rule to response: neuronal processes in the premotor and prefrontal cortex. J Neurophysiol 90:1790-1806.

Wise SP, di Pellegrino G, Boussaoud D (1996) The premotor cortex and nonstandard sensorimotor mapping. Can J Physiol Pharmacol 74:469482.

Wise SP, Boussaoud D, Johnson PB, Caminiti R (1997) Premotor and parietal cortex: corticocortical connectivity and combinatorial computations. Annu Rev Neurosci 20:25-42.

Wolpert DM, Goodbody SJ, Husain M (1998) Maintaining internal representations: the role of the human superior parietal lobe. Nat Neurosci 1:529-533.

Zhang J, Riehle A, Requin J, Kornblum S (1997) Dynamics of single neuron activity in monkey primary motor cortex related to sensorimotor transformation. J Neurosci 17:2227-2246. 


\section{Supplemental Material}

Supplemental Material 1 - Control of latency results for effect size

Inhomogeneity of average spike rate or effect size, e.g. differences in the neural response selectivity, could bias latency measures between different cortical areas. First, since our recruitment curves focused on only the significant neurons, different firing rates in both area could lead to a higher number of significantly tuned neurons in the area with higher firing rates due to statistical power. We analyzed firing rates in PMd and PRR on average across those neurons, which contributed to the analyses in the main text, i.e. neurons with motor-related tuning and no cue-response. For each neuron the average firing rate was determined in the same time window as was used for computing the maximum direction (MD), which was 200 to $350 \mathrm{~ms}$ after onset of the cue (precue in RS, go-cue in N, R, S). Firing rates in PMd during anti-trials were not significantly different from those in PRR in either cueing condition. Second, the effect size in each area was quantified with the maximum p-value for directional selectivity within the analysis time window averaged across all neurons (The negative logarithm of the $\mathrm{p}$ value to the basis of ten was used for statistical comparisons; $\mathrm{p}$ values smaller than $10^{-6}$ were set to $10^{-6}$ ). There was either no significant difference in effect size between PMd and PRR (RS-pro, R-anti, S-pro) or the effect size was significantly higher ( $\mathrm{p}<0.05$, t-test) in PRR compared to PMd (RS-anti, N-pro, N-anti, R-pro and S-anti). Only in RS-anti the effect size in PMd was higher than in PRR. If at all, higher effect size in PRR should lead to opposite latency differences between both areas in most cueing conditions than the ones we found. Taken together, neither differences in average firing rate nor in average tuning strength could explain the observed LDs between PMd and PRR.

In the standard analysis neurons were included if they showed motor-related tuning at some point in time during the analysis time window. The significance criterion of being tuned $(\mathrm{p}<0.05$ in Kruskal-Wallis test) implies an artificial threshold. As additional control we ran our analysis with different thresholds for the significance of an individual neuron's tuning ( $\alpha=0.01,0.1$, or 0.2 ). In the S-pro condition the effect 
of LD(PRR-PMd), which only showed a non-significant trend $(\mathrm{p}=0.057)$ in the standard analysis with tuning threshold $\alpha=0.05$ (Fig. 3B and 6A), was significant ( $\mathrm{p}$ $=0.026$ ) when neurons were selected with a stricter significance criterion for tuning ( $\alpha=0.01$ ). In all other conditions the significances did not change (Fig. S-1). These findings corroborate our conclusions of the main manuscript, especially they provide evidence for earlier motor-goal representations in PMd than PRR in the S-pro condition.

Supplemental Material 2 - Control of latency results for sampling bias

Similar to effect size (Supplemental Material 1) differences in the size of the neuron sample between the two areas could affect latency measures based on recruitment curves. We randomly subsampled the same number of neurons in PMd and PRR (Pesaran et al. 2008), to balance the number of neurons analyzed in each area (Fig. S2). The distributions of LDs in the different task conditions obtained from repeated random sampling of neurons were not different from the original values $(p>0.4$, confidence interval), which means the results were independent of the specific neuron samples. In summary, section 1 and 2 of Supplementary Material suggest that population size, effect size, or average spike rate differences did not confound our latency measures.

Supplemental Material 3 - Alternative latency measures

As an alternative latency measurement we compared the cumulative sums over all single-unit motor-related tuning onset latencies (Fig. S-3). Within each cueing condition and for each neuron the onset latency was defined as the first time point at which the tuning became motor-related and continued to do so for at least $90 \%$ of 50 consecutive time bins (equivalent to the definition of motor planning activity in the memory period of the RS condition; see Material and Methods). The time window of analysis for each cueing condition was identical to the standard analysis in the main manuscript, which was $-200 \mathrm{~ms}$ to $+450 \mathrm{~ms}$ relative to the onset of the pre-cue (RS) 
or go-cue $(\mathrm{N}, \mathrm{R}, \mathrm{S})$. The number of neurons which fulfilled the criterion for motortuning is provided in the legend of Fig. S-3. Median onset latencies for motor-tuning were lower in PMd than PRR ( $\mathrm{p}<0.01$, Wilcoxon signed rank test) in all four cueing conditions which required spatial remapping (RS-anti, N-anti, R-anti, S-pro) These results confirm the results in the main text.

\section{Supplemental Material 4 - Temporary bimodal tuning properties}

Population PSTHs around the time of the go-cue in the N condition (Fig. 2C) suggest that for a brief period of time, between approx. 100-250 ms after cue onset, neural activity in PMd did not only increase for reaches to the maximum direction (MD), but also for reaches to the non-preferred direction (NP). As a consequence, the separation between MD and NP PSTH curves occurred later in PMd than PRR during proreaches, while the recruitment curves and statistical tests indicated synchronous onset of motor-goal tuning in PMd and PRR. Here we explain this seeming contradiction by showing that in PMd in response to the go-cue in the $\mathrm{N}$ condition a small fraction of neurons temporarily showed bimodal tuning, i.e. they briefly reflect both potential motor goals, the pro- and the anti-goal.

To test the significance of directional tuning in our standard analysis in the main text we used a non-parametric 1-way ANOVA with the additional criterion that the length of the normalized DTV had to be larger than 0.2. With this criterion we excluded neurons with a bipolar tuning close to symmetric (ANOVA significant, $|\mathrm{DTV}|<0.2$ ) from the recruitment curves and statistical analysis, but not from the population PSTHs. Such symmetric bipolar tuning could represent two potential motor goals instead of one selected motor goal and would be characterized by a significant ANOVA while the DTV has a length close to zero (see Material and Methods). The test for bimodality was conducted separately in pro- and anti-trials for all neurons which did not have a cue-related response. Figures S-4 A and B show the timeresolved number of bimodal neurons in PMd and PRR for the RS and N conditions. Especially in the $\mathrm{N}$ condition in PMd there was a clear increase in the number of bimodal neurons between 100-250 ms after cue onset, most pronounced for proreaches. Figure S4 C shows the tuning between 100-250 ms for PMd neurons in the 
$\mathrm{N}$-pro condition (normalized within each neuron and averaged across neurons), which showed bimodal tuning for at least 10 time bins in this time window. The fact, that the average tuning shows opposing local maxima at 0 and 180 deg is consistent with the interpretation that the tuning reflects the encoding of two potential motor goals.

We speculate that bimodal tuning was stronger in the $\mathrm{N}$ condition than in the RS condition because of the time pressure for the monkey in the $\mathrm{N}$ condition. It takes longer to process the rule information than the spatial information (compare RTs for R and S conditions in pro-reaches, Fig. 6A). Evaluation of the spatial cue before the rule is known narrows down the choices to two out of four remaining spatial targets, the pro- and the anti-goal. Once the rule information was processed, one of the two putative activity peaks had to be suppressed (resulting in the motor-goal tuning of which we analyzed the latencies with our recruitment curves). This strategy might have been faster for the monkey than building up a new activity peak after the delayed evaluation of the transformation rule. In the RS condition after the pre-cue, there was no time pressure for the monkey and therefore no need to build up potential motorgoal tuning. 
Figure S-1

A: $\alpha=0.2$
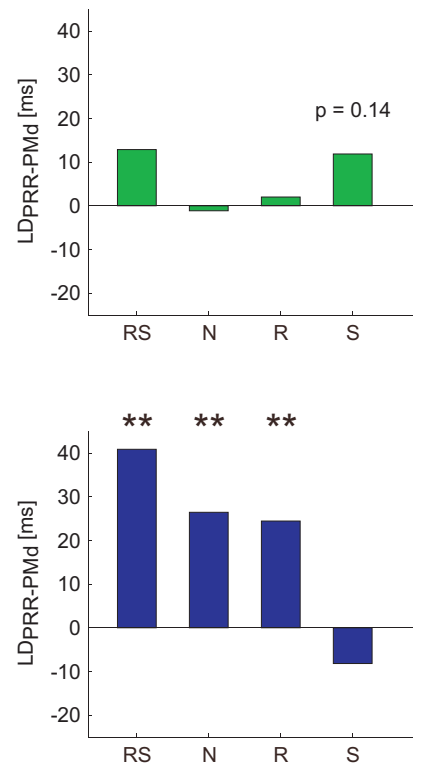

$\mathrm{B}: \alpha=0.1$
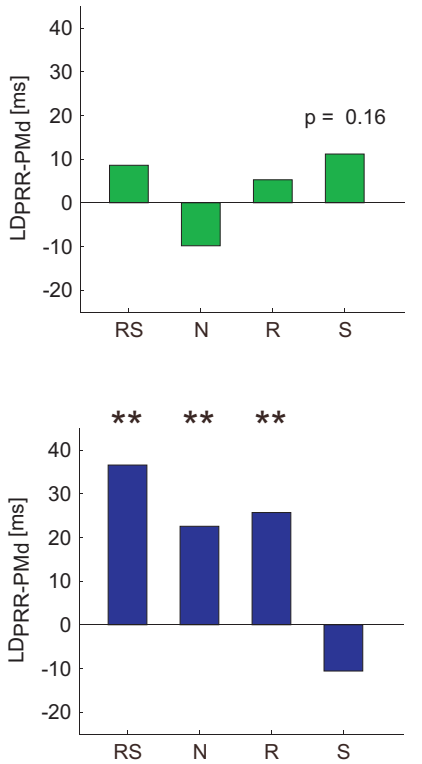

C: $\alpha=0.01$
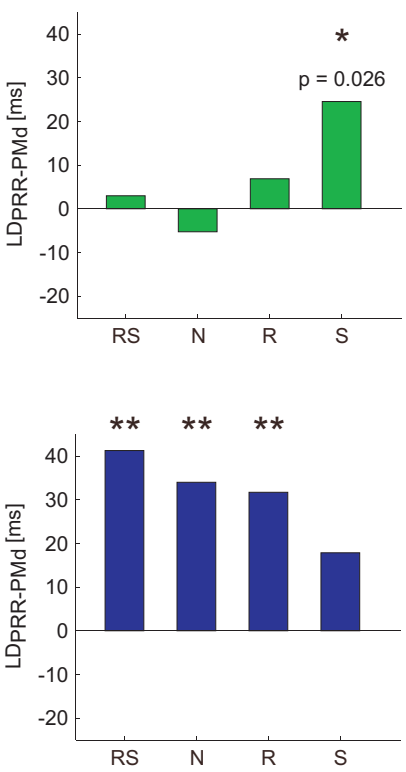

Figure S-1: Control for the effect of tuning significance thresholds on LD measures. Frontoparietal LDs are shown for pro- (top row) and anti-trials (bottom row) in the different cueing conditions. A-C: LD results when different significance thresholds were applied to the spatial tuning of each neuron in proand anti-reaches. A: LDs if significance of spatial tuning in single neurons and time windows was defined by $p<0.2$ (Kruskal-Wallis). B and C: Same as A but significance defined with $p<0.1$ and 0.01 , respectively. 
Figure S-2

\section{Equalization of number of neurons in PMd and PRR}
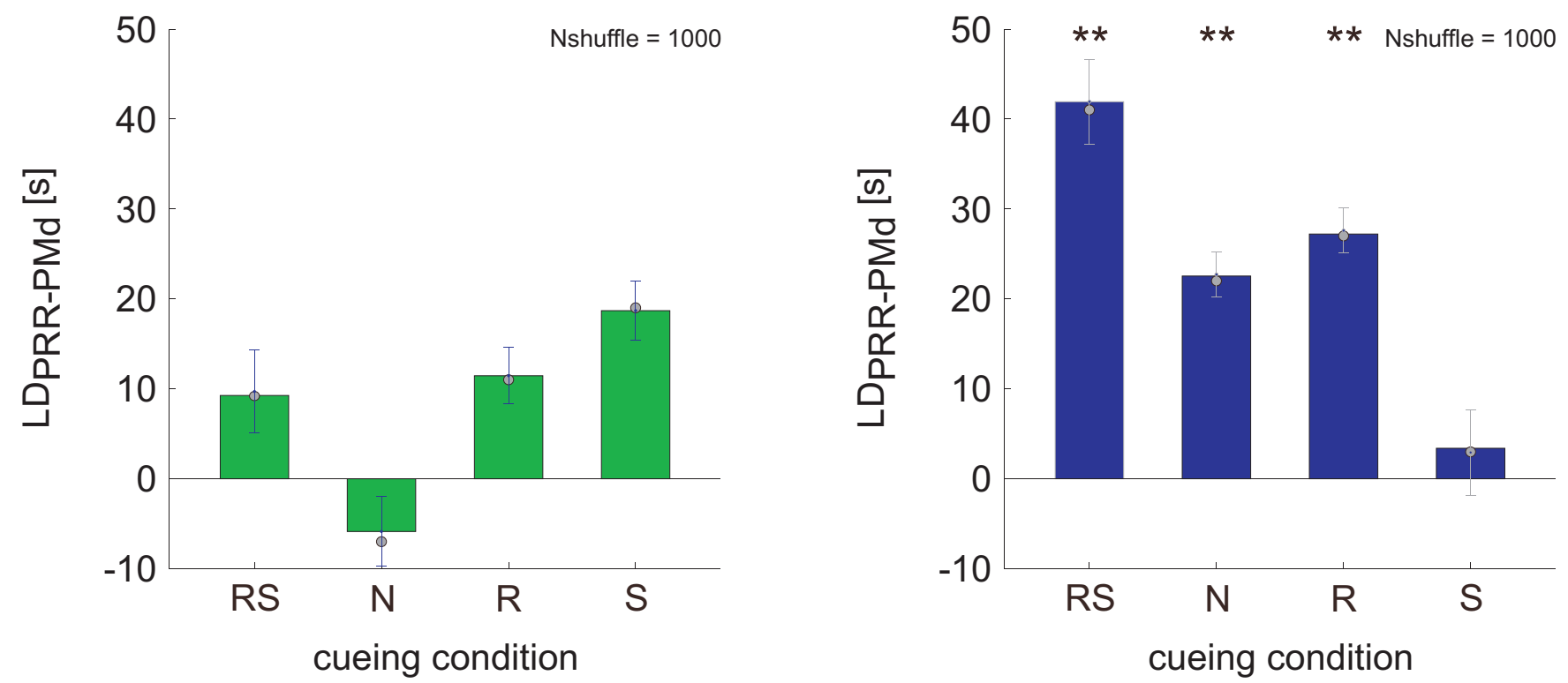

Figure S-2: Control for the effect of sample sizes in PMd and PRR on LD measures. Mean and standard deviation (1000 randomizations) of frontoparietal LDs for pro- (left panel) and anti-trials (right panel) in the different cueing conditions. In each randomization run a random sub-sample of the same number of neurons in PRR and PMd were taken. Asterisks indicate the level of significance $\left({ }^{*}: p<0.05,{ }^{* *}: p<0.01\right)$. The dots indicate the original value with unequal number of neurons in PMd and PRR. The original LDs were not different from the LDs derived from balanced sample sizes $(p>0.4)$. 
Figure S-3

PRO

A: Rule \& Spatial

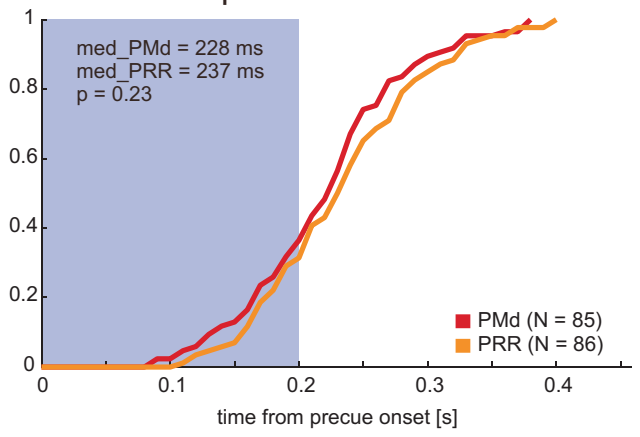

B: Null

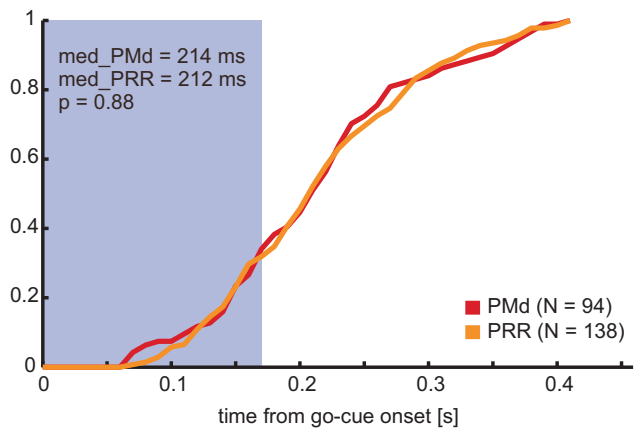

C: Rule only

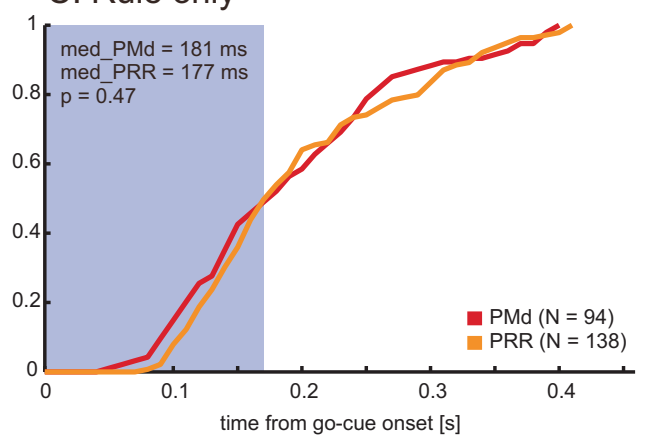

D: Spatial only

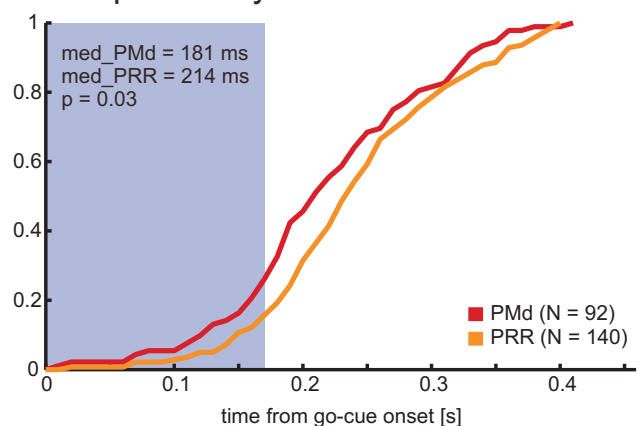

ANTI
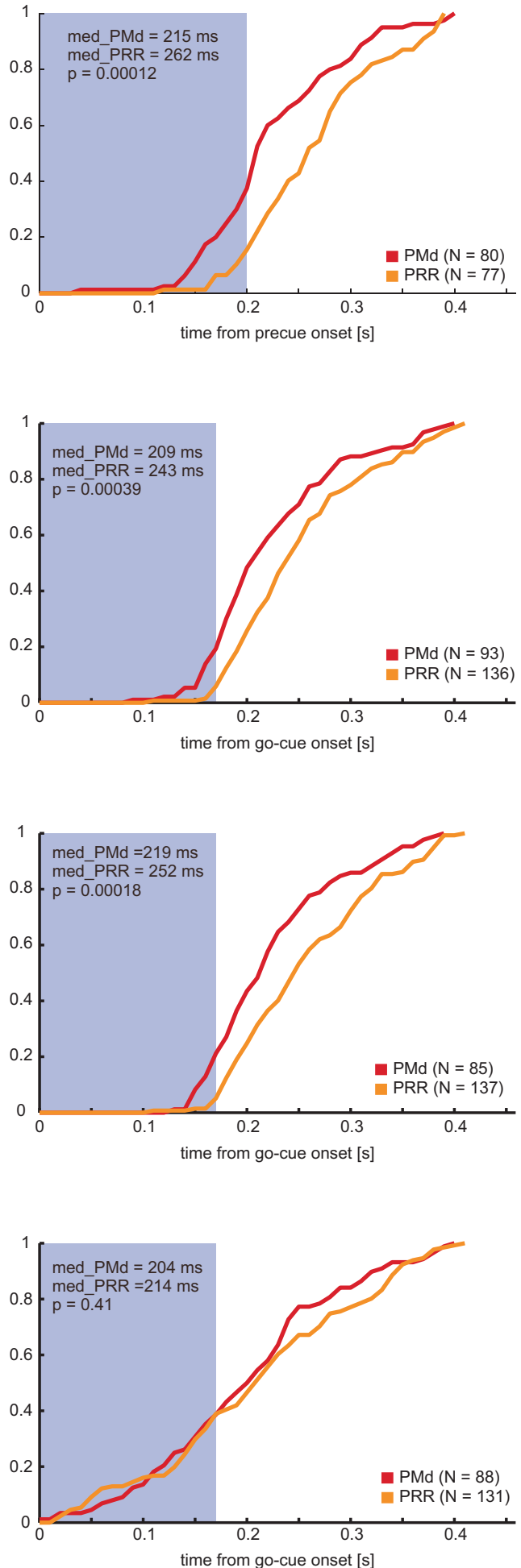

Figure S-3: Alternative neural latency measure. The curves show cumulative sums over the onset latencies of motor-related tuning. PMd (dark) and PRR (light) data are shown separately for pro- (left) and anti-trials (right) in the different cueing conditions (A-D). The motor-tuning onset latencies are derived for each single neuron within each condition. Additional to the cumulative sums the median onset latency and the p-value (ranksum-test) for the comparison between PMd and PRR onset latency distribution are provided. Also, the numbers of neurons in each area are provided, for which an onset latency could be computed. 
Figure S-4

PRO

A Rule \& Spatial
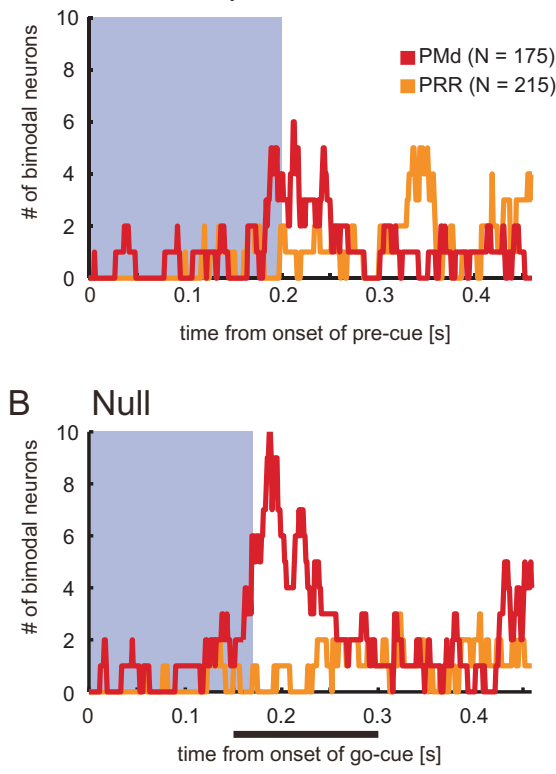

ANTI
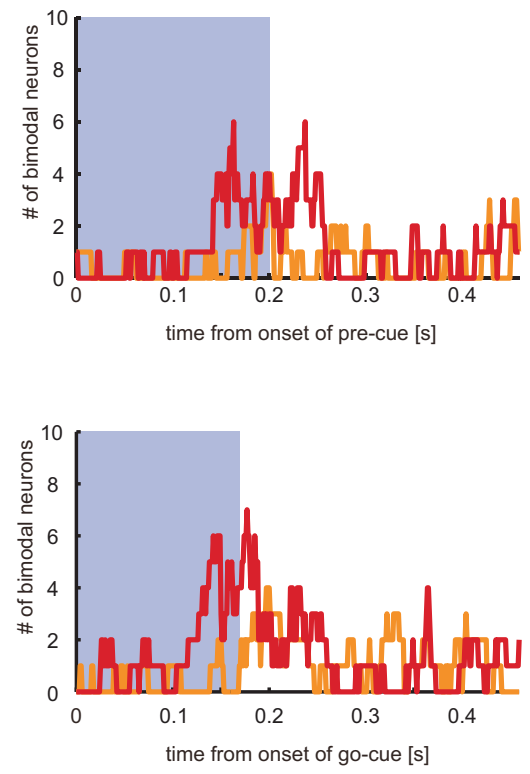

C Null

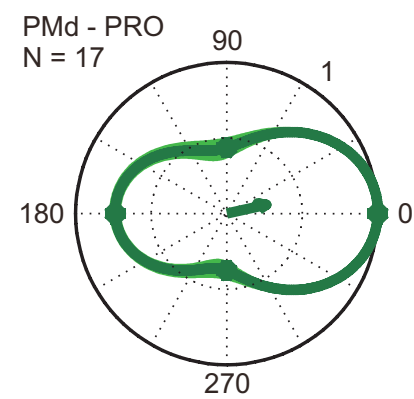

Figure S-4: Analysis of temporary bimodal tuning after cue presentation. A: Number of putatively bimodal neurons in PMd (dark) and PRR (light) and separate for pro- (left) and anti-trials (right) in the RS-condition after onset of the precue. B: Same as in A, but in the N condition after onset of the go-cue. C: Average normalized tuning of putatively bimodal PMd neurons in pro-trials in the $\mathrm{N}$ condition (the condition with the strongest indication for bimodal tuning). The tuning curve is bi-lobed, indicating bimodal tuning, even though not fully symmetrically. The time window, which was used for computing the directional tuning, is indicated by the black bar in B. Data are aligned and normalized to the average maximal response in pro-trials in the time window of analysis. 


\title{
Choosing Goals, Not Rules: Deciding among Rule-Based Action Plans
}

\author{
Christian Klaes, ${ }^{1,2}$ Stephanie Westendorff,, ${ }^{1,2}$ Shubhodeep Chakrabarti, ${ }^{2}$ and Alexander Gail1,2,* \\ ${ }^{1}$ Bernstein Center for Computational Neuroscience, Göttingen, Germany \\ ${ }^{2}$ German Primate Center, Kellnerweg 4, 37077 Göttingen, Germany \\ ${ }^{*}$ Correspondence: agail@gwdg.de \\ DOI 10.1016/j.neuron.2011.02.053
}

\section{SUMMARY}

In natural situations, movements are often directed toward locations different from that of the evoking sensory stimulus. Movement goals must then be inferred from the sensory cue based on rules. When there is uncertainty about the rule that applies for a given cue, planning a movement involves both choosing the relevant rule and computing the movement goal based on that rule. Under these conditions, it is not clear whether primates compute multiple movement goals based on all possible rules before choosing an action, or whether they first choose a rule and then only represent the movement goal associated with that rule. Supporting the former hypothesis, we show that neurons in the frontoparietal reach areas of monkeys simultaneously represent two different rule-based movement goals, which are biased by the monkeys' choice preferences. Apparently, primates choose between multiple behavioral options by weighing against each other the movement goals associated with each option.

\section{INTRODUCTION}

When passing the ball to a player of his team, a soccer player can identify and select the proper target among many potential targets by the color of the jerseys. In this situation the physical targets are identical to potential targets of action (Figure 1A, left). However, when a striker is approaching the opponent goal, multiple alternative action goals have to be inferred from a single physical target (the goal keeper) via spatial transformation rules (Figure 1A, right). The striker might want to aim for the goal keeper, speculating that he or she will jump away, or for the opposite corner of the goal, hoping that the keeper stays. Recently, a lot has been learned on how primates represent and decide between multiple physical targets in target-selection tasks, and how different frontal and parietal cortical areas contribute to target valuation and selection (Sugrue et al., 2005; Gold and Shadlen, 2007; Churchland et al., 2008; Rangel et al., 2008; Andersen and Cui, 2009; Kable and Glimcher, 2009; Kim and Basso, 2010; Bisley and Goldberg, 2010; Cisek and Kalaska, 2010). Little is known, however, about decision processes in rule-selection tasks, which require choosing among goals based on a spatial transformation rule (Tremblay et al., 2002), and in which alternative goals might not be physically present as target stimuli, but have to be spatially inferred, like in the example of the striker.

In rule-selection experiments, alternative movements are conducted under identical spatial sensory conditions, but according to different context-defined transformation rules (Wise et al., 1996; Wallis and Miller, 2003). In antisaccade or antireach tasks (Figure 1A, right) a single visuospatial input is associated with two alternative movement goals: one that is directly cued by the sensory input (aim at the keeper), and another that has to be inferred from a spatial cue by applying a remapping rule (aim at the corner of the soccer goal opposite to the keeper) (Crammond and Kalaska, 1994; Shen and Alexander, 1997; Schlag-Rey et al., 1997; Everling et al., 1999; Zhang and Barash, 2004; Medendorp et al., 2005; Gail and Andersen, 2006). Two alternative decision processes are conceivable in such ruleselection tasks. The sensorimotor system could first choose among the alternative rules, and then only compute one sensorimotor transformation to encode the single motor goal that is associated with the selected rule (rule-selection hypothesis). Alternatively, the system could first compute all potential sensorimotor transformations, and then select among the multiple resulting motor-goal options (goal-selection hypothesis).

The difference between the rule- and goal-selection hypotheses should become obvious in areas of the brain that have "spatial competence" for movement planning, i.e., areas that exhibit spatially selective neural encoding of motor goal information. This is the case, for example, in the premotor cortex (Weinrich and Wise, 1982; Snyder et al., 1997; Crammond and Kalaska, 2000) and the posterior parietal cortex (Mountcastle et al., 1975; Snyder et al., 1997; Batista et al., 1999; Gail and Andersen, 2006). The rule-selection hypothesis predicts that such areas only encode one goal at a time, according to the preliminarily selected rule, but not multiple rule-based potential goals simultaneously (Figure 1B, left). The goal-selection hypothesis predicts that they simultaneously encode all alternative potential movement goals prior to the decision (Figure 1B, right). Therefore, the two hypotheses are distinguishable only at predecision stages, where the simultaneous existence of multiple, alternative, potential motor goals in a rule-selection task would favor the goal-selection hypothesis.

Evidence for potential motor goal encoding in spatial rule selection tasks, i.e., in situations like in the example of the striker, is lacking. Several areas of the brain have been thought 
A

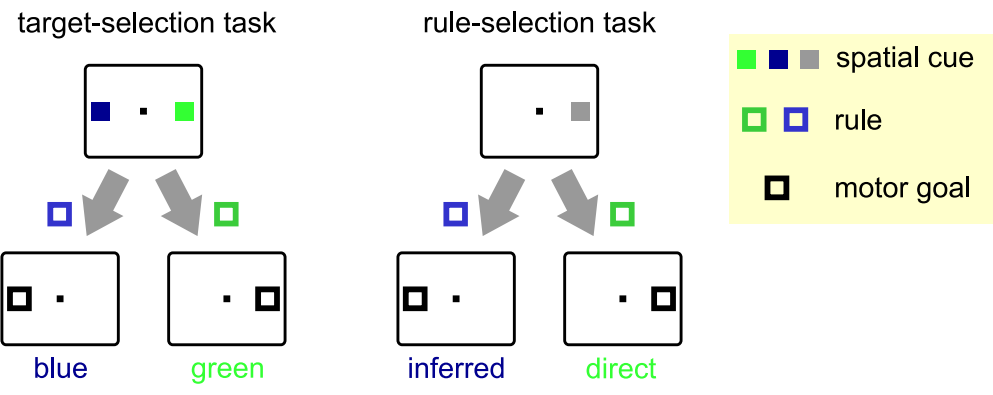

B

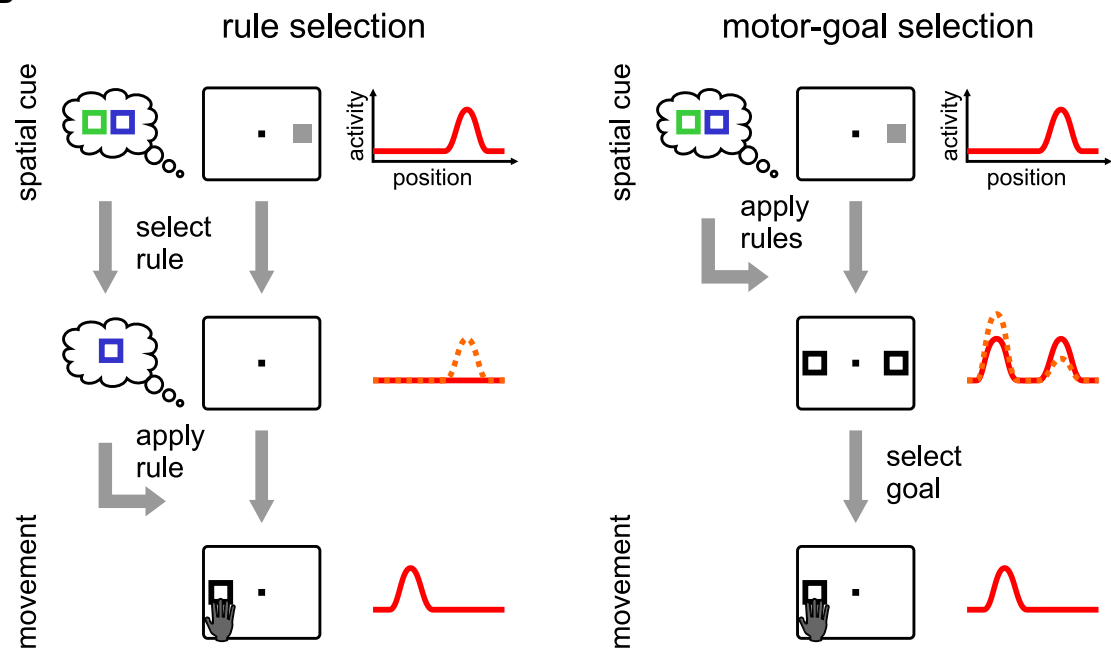

Figure 1. Target-Selection versus RuleSelection Task

(A) In a target-selection task (left) two distinguishable (e.g. colored) spatial cues (targets) are presented, of which one should be chosen as movement goal according to a selection rule. Both potential targets have been spatially specified before the selection. In our rule-selection task only one spatial cue is provided, while the goal of the movement can be the position of the spatial cue (direct rule) or diametrically opposite to it (inferred rule).

(B) Neural rule- or goal-selection encoding in a rule-selection task. Decisions in ambiguous ruleselection tasks might follow a "rule selection" hypothesis (left columns). The rule will first be selected, and then be applied to the spatial cue to compute the single associated spatial motor goal. In spatial planning areas there will be either no spatial information encoded (solid red), or a memory of the spatial cue location (dotted orange) prior to the final selection. The "motor-goal selection" hypothesis (right columns) states that both alternative rules are applied to the spatial cue to create two competing spatial representations for the direct and the inferred motor goal, which may (dotted orange) or may not (solid red) be modulated by choice preferences of the subject. Note that the initial spatial encoding during the spatial cue presentation and the postdecision encoding of the final motor goal are identical in both hypotheses. to encode multiple potential motor goals in space, but only in experiments involving selection among multiple physical targets (Basso and Wurtz, 1998; Cisek and Kalaska, 2005; Lau and Glimcher, 2008). However, in such tasks, multiple alternative spatial representations in the neural activity could be associated with multiple physical targets rather than motor goals. Therefore, target selection tasks are unsuitable for distin- reach planning in a novel rule-selection task (Figure 2). We show that two spatial, rule-based potential motor goals can be simultaneously encoded, supporting the goal-selection hypothesis.

Potential motor goals can encode all alternative choices as defined by the task (options), or biased representations of all choices based on previous reward experience (preferences),

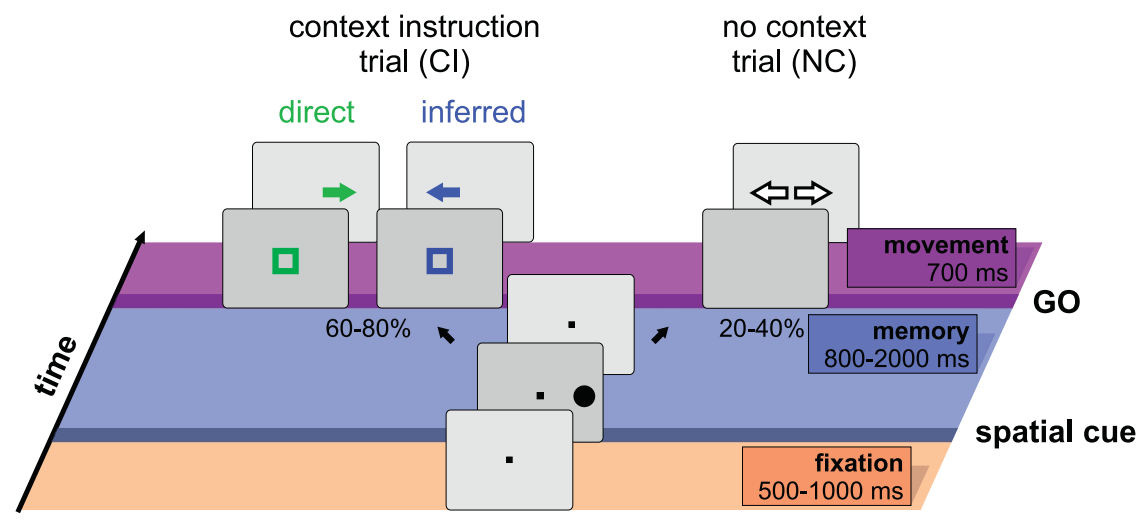

Figure 2. Rule-Selection Task with Instructed Delay

In our potential motor-goal (PMG) task a single spatial cue was presented at one of the four cardinal directions prior to a memory period. $60 \%-80 \%$ of the trials were context-instruction trials (PMG-Cl) in which a contextual cue was shown in a second cue period ("GO"). This contextual cue instructed a direct reach toward the position of the preceding spatial cue (green) or an inferred reach toward the diametrically opposite direction (blue). In $20 \%-40 \%$ of the trials no contextual cue was shown (PMG-NC), and the monkeys were free to choose the goal either according to the direct or inferred transformation rule. 
depending on which stage of the decision process they represent. So far, empirical evidence for preference encoding has been lacking for skeletomotor tasks, even in target selection experiments. Many previous oculomotor studies showed modulation of neural target responses by choice probability or some form of value assignment (preference encoding) in different brain areas of monkey (Basso and Wurtz, 1998; Dorris and Munoz, 1998; Platt and Glimcher, 1999; Sugrue et al., 2004; Dorris and Glimcher, 2004; Yang and Shadlen, 2007; Lau and Glimcher, 2008; Kim and Basso, 2010; Louie and Glimcher, 2010) and human (Hampton et al., 2006; Kable and Glimcher, 2007; Yanai et al., 2008; Wunderlich et al., 2009). Target-selection experiments using skeletomotor behavior, like reaching, showed encoding of freely selected targets in the parietal reach region (PRR) (Scherberger and Andersen, 2007; Pesaran et al., 2008), and potential motor goal encoding in the dorsal premotor cortex (PMd) of monkeys (Cisek and Kalaska, 2005), but the task designs in these studies did not allow dissociation of option versus preference encoding. We tested for the encoding of options versus preferences by using a mixture of instructed and free-choice trials in combination with different probabilistic reward schemes. We show that potential motor goal signals in monkey parietal and premotor cortex during reach planning represent mostly choice preferences, rather than options or preliminary selections.

\section{RESULTS}

\section{Balanced Choices between Alternative Rule-Based Motor Goals}

To distinguish between the rule- and goal-selection hypotheses we first tested if two potential rule-based motor goals can be encoded simultaneously, since this would provide evidence for the goal-selection hypothesis (Figure 1B, right). We designed a potential motor goal (PMG) task, in which subjects had to choose between two rule-based motor goals in each trial, and characterized the spatial selectivity of neural activity as a function of the spatial motor goal(s) during ambiguous reach planning.

Two male rhesus monkeys were trained to perform a memoryguided antireach task with instructed delay (Figure 2). A single spatial cue was combined with an optional contextual color cue. The contextual cue defined one of two spatial transformation rules according to which the spatial cue had to be mapped onto the associated motor goal. The reach goal could either be identical to the spatial cue (direct reach) or opposite to it (inferred reach). In each trial of this PMG task, both options were available in parallel to the subjects during reach planning, since the contextual cue was presented only at the end of the instructed delay, while the spatial cue was presented prior to the delay.

The PMG task consisted of two randomly interleaved trial types, either with context instruction at the end of the delay period (PMG-Cl, 60\%-80\%), or without context instruction (no context, PMG-NC, 20\%-40\%). We used the "free-choice" PMG-NC trials to probe the subjects' behavioral choice preferences, and manipulated the subject's choice preferences by varying the reward schedules (see below).

The performance in PMG-Cl trials was high for both monkeys (PMG-Cl: $88 \pm 1 \%$ [monkey A], $80 \pm 1.4 \%$ [monkey S]). Most errors could be attributed to ocular fixation breaks, while improper choices in instructed trials, i.e., confusions of the direct and inferred reach goal, were rare in both monkeys $(<2 \%)$. In the PMG-NC trials, both direct- and inferred-goal choices with proper ocular fixation and timing were considered correct, while reaches to any other directions were considered incorrect. In the first data set we are going to present, correct PMG-NC trials were rewarded according to a bias minimizing reward schedule (BMRS).

The BMRS was designed to reinforce balanced choice behavior, by taking the reward history of the monkey into account and reducing the reward probability if the behavior was biased. In the BMRS, random behavior with an equal amount of choices for either motor goal (on a short-term average across few trials) leads to $50 \%$ reward probability, while any consistent bias in choices leads to lower reward probabilities (see Experimental Procedures). With the BMRS, the direct choices $(40 \pm 0.1 \%$, monkey $A ; 39.4 \pm 2.5 \%$, monkey $S)$ and inferred choices $(48.7 \pm 0.1 \%$, monkey $A ; 44.9 \pm 2.6 \%$, monkey $S)$ were mostly balanced, with only a small bias in favor of inferred choices (Figure 3A). The overall balance between direct and inferred reach choices in PMG-NC trials suggests that the monkeys had close-to-equal preference for the two potential motor goals in BMRS sessions (= balanced data set).

\section{Rule Selection versus Goal Selection-Potential Motor Goal Encoding}

According to the goal-selection hypothesis, the planning of two equipotent alternative actions should lead to the neural encoding of both corresponding motor goal representations simultaneously. According to the rule-selection hypothesis, we would have to expect only one motor goal representation at a time despite balanced behavioral choices on average (Figure 1B). In the balanced choice condition, we recorded 145 (66 [A], 79 [S]) neurons in PRR, of which 97 (67\%; 49 [A], 48 [S]) fulfilled the criteria to be tested for the encoding of potential motor goals (see Experimental Procedures). For the purpose of separating the rule-selection from the goal-selection hypothesis PMG-Cl and PMG-NC trials were analyzed jointly, since the trial types are indistinguishable and unpredictable to the subjects prior to the optional contextual cue at the time of the GO signal.

Figure 3B shows an example neuron from PRR with a bimodal spatial selectivity profile from the balanced data set in the PMG task. We first tested the neurons spatial selectivity in two reference conditions. In the definite motor goal (DMG) task the monkeys were unambiguously instructed about the pending motor goal prior to memory period, i.e., the spatial and the contextual cue were shown at the beginning of the memory period (see Experimental Procedures). During such unambiguous planning in the DMG task, the neuron's responses reflected the unique downward motor goal in the "direct" (Figure 3B, left) and "inferred" (Figure 3B, center) context. This is indicated by the selectivity profiles for direct and inferred reaches that show the neural response as a function of the cue position, and that are shifted by $180^{\circ}$ relative to each other (Figure 3B, bottom). Such motor-goal selectivity is characteristic for PRR (Gail and Andersen, 2006; Gail et al., 2009), and common to most directionally selective neurons of the current study (>80\% across 
A

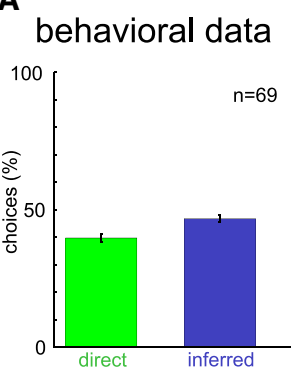

B
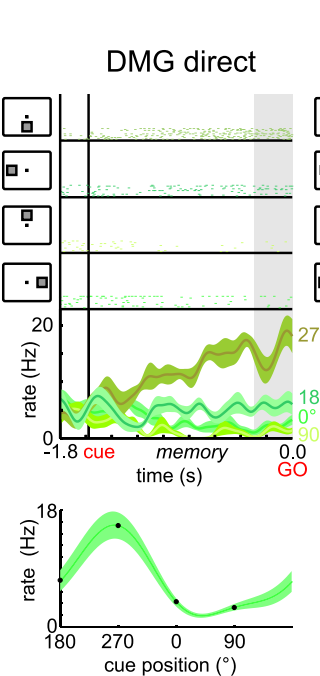

single cell example DMG inferred
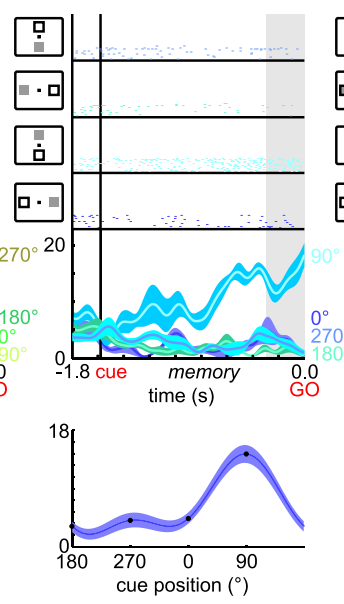

PMG
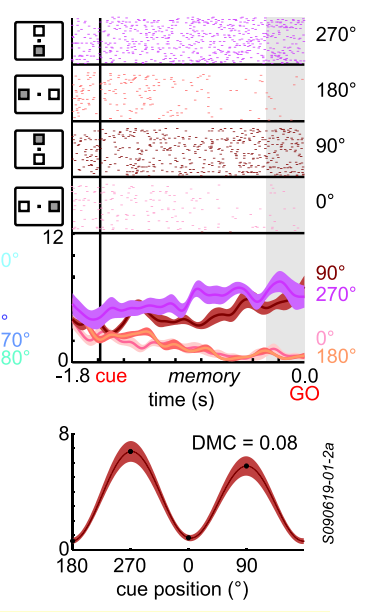

- cue $\square$ (potential) motor goal

C

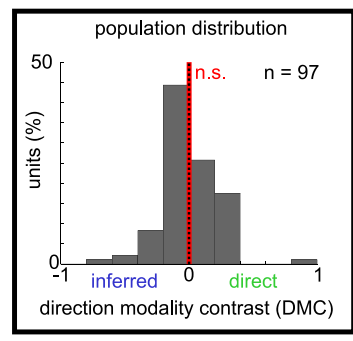

population activity

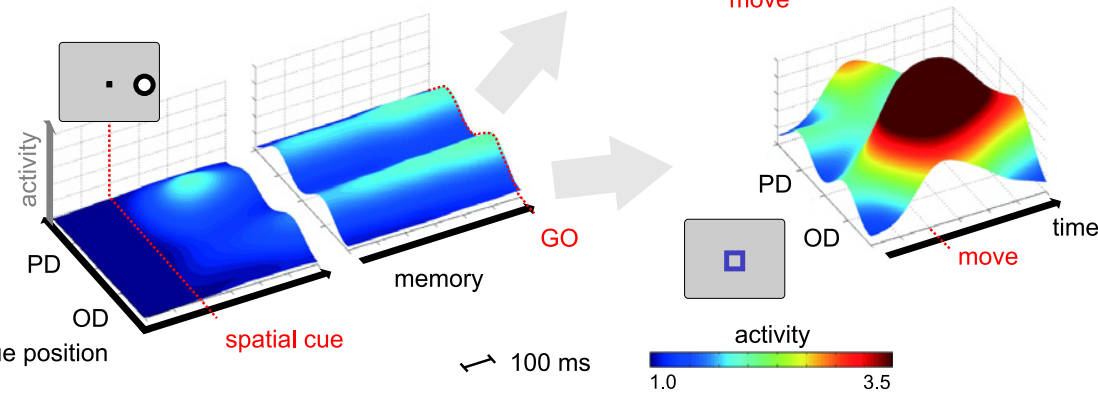

Figure 3. Neural Encoding of Potential Motor Goals in a Data Set with Balanced Choice Behavior

(A) Balanced percentage of direct (green) and inferred (blue) choices in PMG-NC trials with a bias minimizing reward schedule (BMRS; $n=$ total number of behavioral testing days for both monkeys; error bars denote SEM).

(B) Response of an example PRR neuron. The top panels show the spike rasters in the definite motor goal (DMG) task with the direct rule (left column), the inferred rule (center) and in the PMG task (right), with average responses for each spatial cue position $\left(0^{\circ}, 90^{\circ}, 180^{\circ}\right.$, and $\left.270^{\circ}\right)$ below. Trials were aligned to the GO signal, while "cue" marks the average onset time of the spatial cue. Pictograms at the side of the spike rasters illustrate the spatial cue positions (filled squares) and the corresponding motor goal positions (open squares) according to the task. The bottom panels show the selectivity profiles of the neuron (average rate) as a function of cue position in the late memory period (shaded time window). Selectivity profiles were interpolated for illustrative purposes only. The shaded curves denote the SEM.

(C) Population results for the balanced data set in PRR. The average normalized activity of all eligible PRR neurons during the PMG-Cl task is shown aligned to the spatial cue onset, the GO signal, and the movement onset (dotted red lines). Selectivity profiles were aligned to the neurons' preferred directions in DMG trials before averaging (PD, preferred direction; OD, opposite-to-preferred direction). Direct-cued and inferred-cued PMG-Cl trials physically differ only at the time of the context instruction, hence, data are plotted jointly for the cue and memory periods. The inset shows the distribution of direction modality contrast (DMC) values for all eligible neurons in the late memory period. 
A

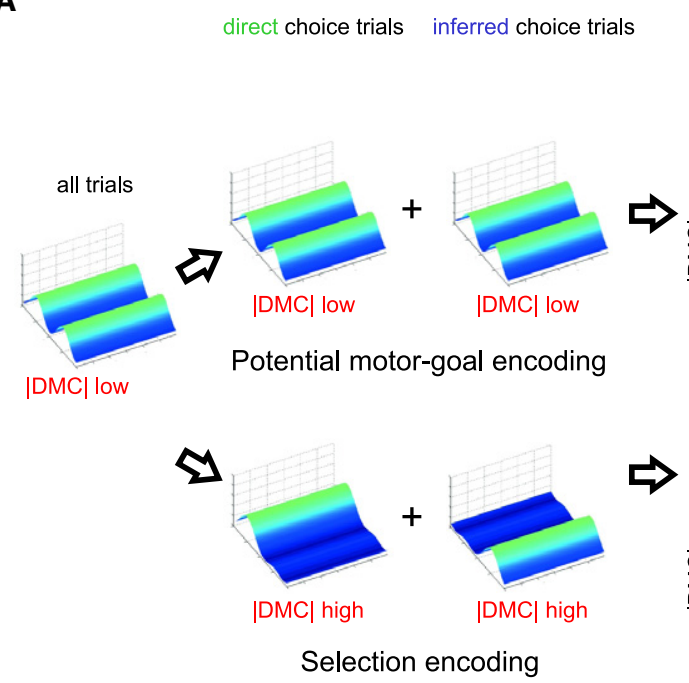

DMC similarity

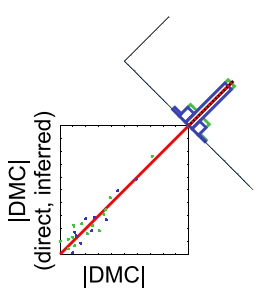

(all)

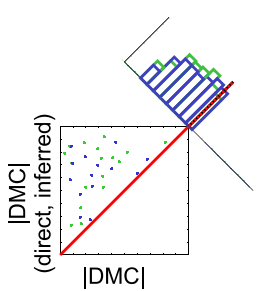

(all)
B

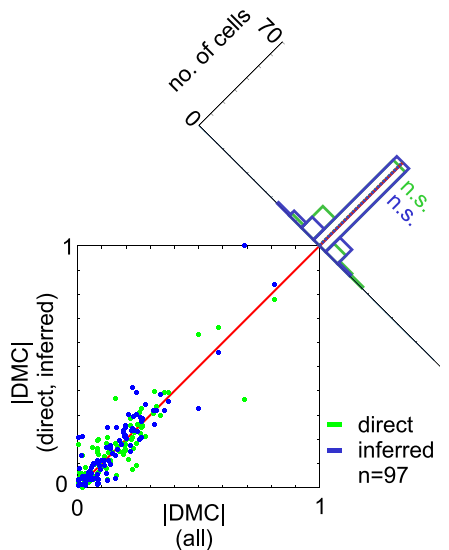

(all)

Figure 4. Encoding of Potential Motor Goals versus Preliminary Selections

(A) Schematic description of how to test the two alternative hypotheses with a choice-selective analysis of the free-choice trials (PMG-NC). Bimodal selectivity in the PMG trials could be the result of averaging across trials with alternating preliminary selection of either of the two potential motor goals (lower row), or reflect potential motor-goal encoding proper, independent of the later choice (top row). The similarity of the absolute DMC values, computed separately for direct-choice (green) and inferred-choice (blue) trials, with the choice-indifferent absolute DMC values, computed for all trials, allows to differentiate the two possibilities (see Figure S2 for a control).

(B) Choice-selective versus choice-indifferent absolute DMC values. The difference histogram shows that there is no significant deviation from the unity line, indicating encoding of two potential motor goals, and contradicting preliminary selection encoding.

data sets). Importantly, in the ambiguous PMG task (Figure 3B, right), the neuron was always most active if the previous spatial cue in a PMG task potentially indicated a downward $\left(270^{\circ}\right)$ reach, i.e., when it had appeared either at the upper $\left(90^{\circ}\right)$ or lower $\left(270^{\circ}\right)$ position. Since the spatial selectivity profile is plotted as a function of cue location, the bimodal activity profile with a peak separation of $180^{\circ}$ indicates encoding of a single motor-goal direction in two different cue conditions, not two different motor-goal directions. Notably, the neuron was not active in trials with right-side $\left(0^{\circ}\right)$ or left-side $\left(180^{\circ}\right)$ cues, but only for those two directions (up and down) that were equally probable to instruct a downward motor goal.

The bimodal response profile of the example neuron in Figure 3B in the PMG task matched the prediction of the goal-selection hypothesis, and contradicts the rule-selection hypothesis. The bimodal profile mimicked the response pattern one would expect when averaging (not summing) the two response profiles in the DMG task. This means, the response pattern during planning of two equipotent alternative potential motor goals was an equally weighted linear combination of the response patterns during unambiguous planning of the two respective unique motor goals. In a model-based analysis we quantitatively confirmed this view (see Figures S1 and S4 available online).

Bimodal selectivity profiles dominated the balanced data set in PRR. The average population activity in the balanced data set shows two stable ridges of activity during the memory period (Figure $3 \mathrm{C}$ ). Since the cue-position axis marks the location of the spatial cue relative to the preferred direction (PD) of each neuron (as measured in the DMG task), the two ridges indicate that on the population level the direct and inferred goals are represented simultaneously during ambiguous reach planning. For quantitative analysis we characterized the bimodal versus unimodal selectivity of each neuron with a direction modality contrast (DMC). Positive DMC indices indicate selectivity for the direct motor goal; negative values indicate selectivity for the inferred motor goal. Indices close to zero indicate symmetric bimodal tuning (not lack of tuning) since only directionally selective neurons were considered (see Experimental Procedures). The mean DMC of the balanced data set did not significantly deviate from zero $(\mathrm{m}=0.001$; standard error of the mean [SEM] $=0.021$, $p>0.05$ ), indicating that in the balanced data set most neurons had bimodal selectivity profiles (Figure 3C, inset).

\section{Potential Motor Goals or Preliminary Selections? Choice-Selective Analysis in PRR}

The existence of a bimodal neural selectivity pattern in the balanced data set is not sufficient to demonstrate potential motor goal encoding. The monkeys could have preliminarily selected one of the two potential motor goals during the memory period in every trial, and randomly switched their selection from trial to trial. Such switching would be obscured in PMG-Cl trials due to the explicit context instruction at the time of the GO cue. The bimodal selectivity pattern revealed by the above analyses would denote an artifact of averaging across inhomogeneous sets of trials in this case (Figure 4A, bottom).

With a choice-selective analysis of the free-choice (PMG-NC) trials we can rule out this possibility. We can instead show that both potential motor goals were encoded independently of the monkey's later choices (Figure 4A, top). If the monkeys made preliminary selections in every trial then this selection should 
be reflected in an unambiguous neural encoding of this preliminary selection in the late memory period immediately prior to the monkeys decision (preliminary selection encoding). We sorted PMG-NC trials according to the choice of the monkey, and computed DMC values of the neural activity in the late memory period separately for the trials in which the monkeys freely chose the direct and inferred goals. If the low DMC of a neuron with bimodal selectivity was the averaging result of two opposite unimodal selectivity profiles, one for direct-choice trials and the other for the inferred-choice trials, then a low absolute value of the original DMC would be attended with high absolute values of the two choice-selective DMC values for this neuron. This means, preliminary selection encoding would be indicated by a low similarity between the original and the choice-selective DMC values across neurons (Figure 4A, bottom). Vice versa, we can reject the selection hypothesis if a neuron in both choice-selective subsets of trials shows a bimodal selectivity pattern, i.e., when low absolute values of the original DMC is attended with low absolute choice-selective DMC values, resulting in a high similarity between original and choice-selective DMC across neurons (Figure 4A, top).

The balanced data set in PRR yielded bimodal selectivity in PMG-NC trials separately within direct-choice and within inferred-choice trials. The absolute choice-selective DMC values for direct- and inferred-choice trials were highly similar to the absolute original DMC values (Figure 4B). This can be seen by the fact that the average distance of the data points from the unity line did not significantly differ from zero neither for direct$\left(p_{d}>0.05\right)$ nor inferred-choice $\left(p_{i}>0.05\right)$ trials. When-as a control - the method was applied to the DMG data set, in which we know that the monkeys had selected the motor goal already during the memory period, then the choice-selective and original DMCs were highly and significantly dissimilar $\left(p_{d}=0.0012, p_{i}=\right.$ 0.00067 ; see Figure S2). Additional variance tests indicated that it is unlikely that the bimodal selectivity profiles were the consequence of rapid switching between two alternative preliminary selections within the time of a trial (Figure S2).

Taken together, the results from the choice-selective analysis of the balanced data set indicated genuine encoding of potential motor goals rather than alternating preliminary selections in PRR. This supports the motor-goal selection hypothesis and argues against the rule-selection hypothesis.

\section{Manipulating Behavioral Choice Preferences}

Depending on which stage of the decision process a brain area belongs to, encoding of multiple potential motor goals in that area could represent the multiple options offered to the subject (the "menu"), or the competing behavioral goals associated with these options and weighted with the subject's preference for either choice. Motor-goal options were defined solely by the task. In any PMG trial two motor-goal options (the direct and inferred motor goal) were valid during the memory period. Encoding of motor-goal options should lead to the representations of two potential motor goals during the memory period of all PMG trials, irrespective of any choice preferences of the monkeys. Motor-goal preferences were defined by the monkeys' average choice behavior in PMG-NC trials. Since the monkeys had close-to-equal choice preferences for direct and inferred motor goals in the balanced data set, the bimodal selectivity profiles are not suited to dissociate encoding of motor-goal options versus motor-goal preferences. If, on the other hand, the monkeys had a bias in favor of one of the two options, then encoding of motor-goal preferences should lead to neural activities in the memory period of PMG trials that reflect the relative probability of selecting either potential goal in the PMG-NC trials. By using different reward schedules we recorded two data sets, one with balanced choice behavior (see above), and one with strong behavioral choice bias, to dissociate the options and preference encoding hypotheses.

In the second data set, correct PMG-NC trials were rewarded according to an equal probability reward schedule (EPRS). With the EPRS, in which a $50 \%$ reward probability independent of the choice history was guaranteed (reward probability: $52 \pm 5 \%$; $p>$ $0.05[\mathrm{~A}], 50 \pm 4 \% ; \mathrm{p}>0.05$ [S]), both monkeys showed a strong bias in favor of the inferred reach goal (Figure 5A), i.e., most reaches in PMG-NC trials were directed toward the inferred motor goal $(85 \pm 4.0 \%$ monkey A, $63 \pm 4.1 \%$ monkey $S)$, and only a small fraction toward the direct goal $(2.4 \pm 0.8 \%$ monkey $A$, $17.8 \pm 3.4 \%$ monkey $S)$. In the remaining PMG-NC trials (12.6\% monkey A, 19.2\% monkey S) the monkeys aborted the trial without reaching, or reached toward one of the orthogonal goals $(<1 \%)$. This means that both monkeys had a preference for the inferred goal when the transformation rule was unknown, and when either goal selection was rewarded with equal probability in EPRS sessions (= biased data set).

We can only speculate about the reason for the intrinsic bias of both monkeys during the EPRS (Figure S3). The reason behind this behavior is not immediately relevant for the purpose of dissociating options encoding from preference encoding at the neural population level, though. It is sufficient to note that both monkeys consistently had a similarly strong bias over an extended period of time in the EPRS sessions, and little to no bias in the BMRS sessions.

\section{Options versus Preference Encoding in PRR}

If neurons encoded behavioral choice preferences then we would expect encoding of only the inferred motor goal in the PMG trials of the biased data set, in contrast to the encoding of both potential motor goals simultaneously as seen in the balanced data set. This should be true in the late memory period of all PMG trials, since PMG-Cl trials are indistinguishable from the PMG-NC trials prior to the GO signal, and were randomly interleaved.

In PRR, the biased data set contained a total of 258 (159 [A], 99 [S]) recorded neurons. A total of 148 (57\%) neurons (96 [A], 52 [S]) of the biased data set fulfilled the criterion for the analysis of potential motor-goal encoding.

The PRR example neuron in Figure 5B was recorded in the biased data set and was most active during planning of leftward $\left(180^{\circ}\right)$ reaches in direct-cued or inferred-cued DMG trials. In PMG trials the neuron was only highly active if the spatial cue was presented at the right side $\left(0^{\circ}\right)$, i.e., as if an inferred instruction had been given externally or had been selected internally. Such unimodal selectivity for the inferred goal dominated the biased data set in PRR. The average normalized population activity showed only a brief response increase when the cue 
A

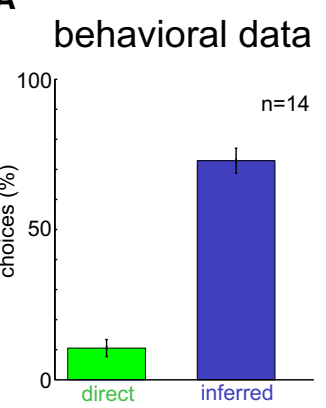

B
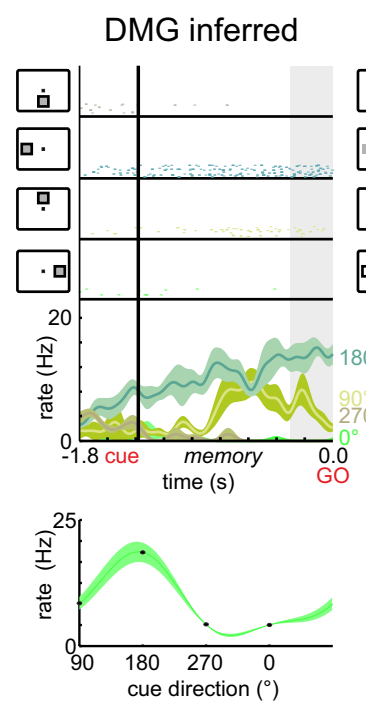

single cell example

DMG direct
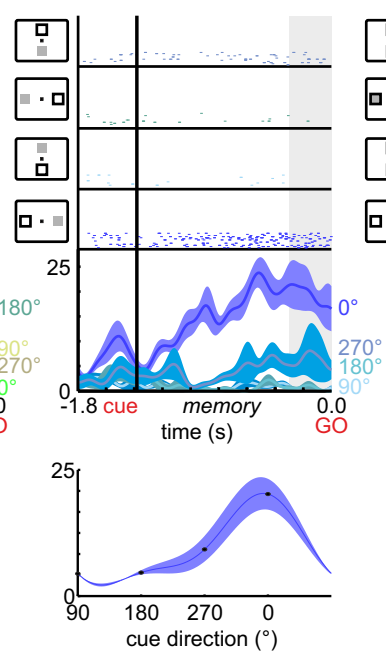

PMG
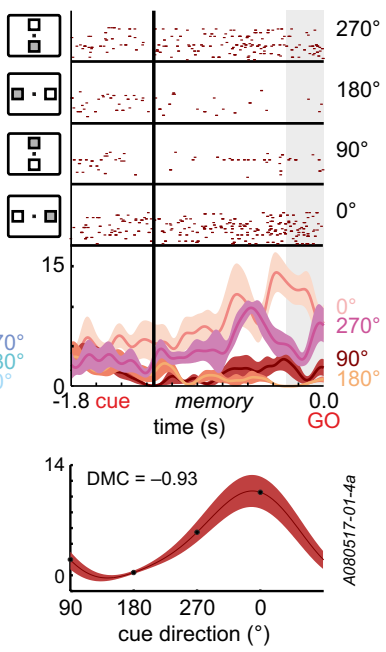

ㅁ (potential) motor goal

C

population activity

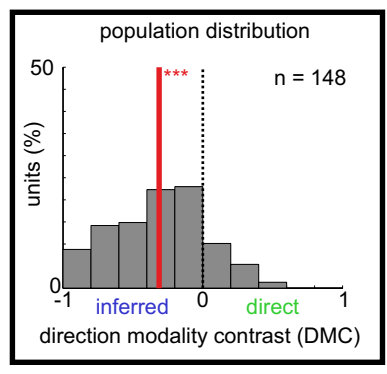

moe

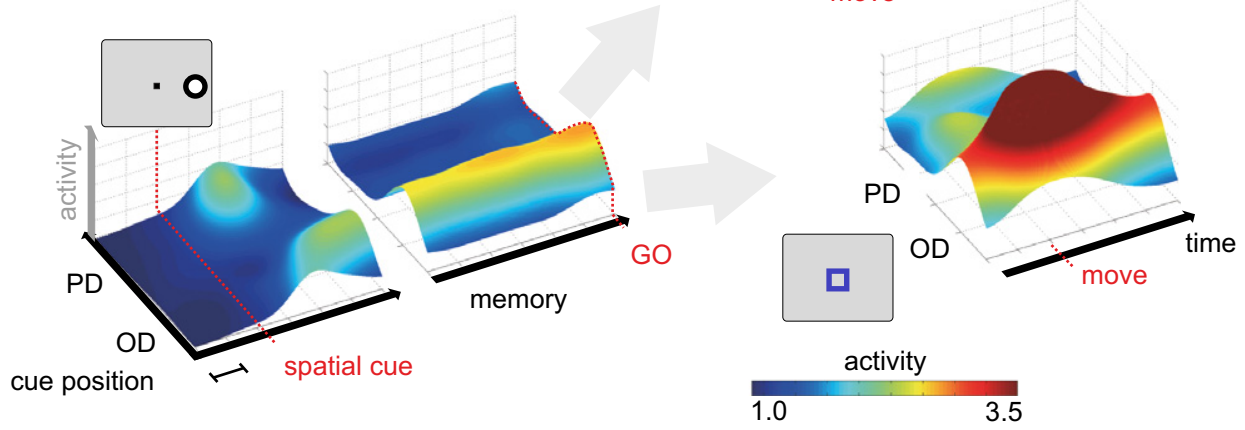

Figure 5. Neural Encoding of Motor Goal Preference in a Data Set with Biased Choice Behavior

(A) Biased percentage of direct (green) and inferred (blue) choices in PMG-NC trials with an equal probability reward schedule (EPRS). Same conventions apply as in Figure 3.

(B) Example PRR neuron from the biased data set. It showed motor-goal selectivity in the DMG task, as did the example in Figure 3. But in the PMG task it is only active if the spatial cue appears opposite to the neurons PD.

(C) Correspondingly, PRR population activity shows a strong representation at the opposite-to-cue position (OD) during the memory period. The DMC distribution is significantly biased for the inferred goal (mean $\mathrm{DMC}=-0.31$; ${ }^{* \star} \mathrm{p}<0.001$; rank-sum test).

matched the preferred direction (PD) of the neurons. This was followed by a high level of activity when the cue was opposite to the PD, corresponding to an encoding of the inferred goal throughout the memory period (Figure $5 \mathrm{C}$ ). The mean DMC during the memory period of the biased data set was negative $(\mathrm{m}=-0.31$; SEM $=0.028)$ and significantly different from zero (rank-sum test, $p<0.001$ ) (Figure $5 \mathrm{C}$, inset). This means that the behavioral preference was reflected in a significant bias of 
A

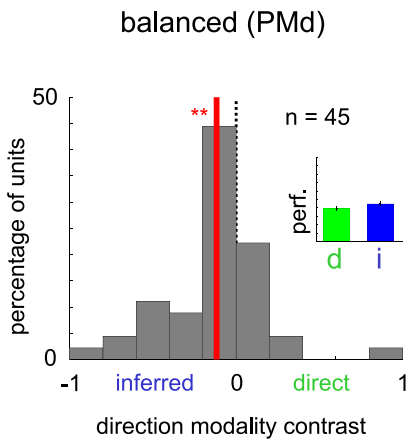

B

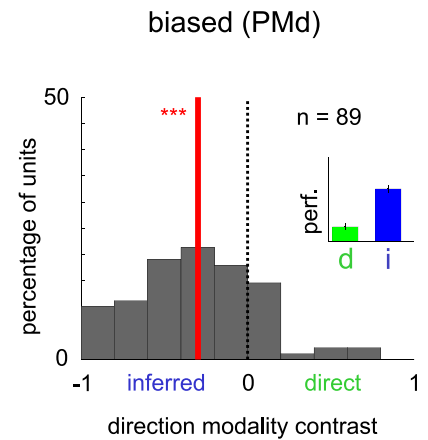

C

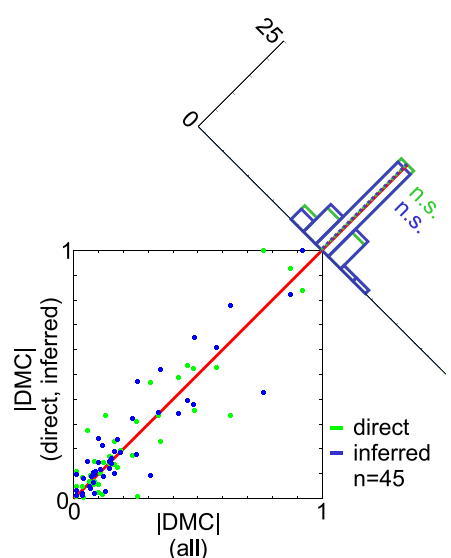

Figure 6. Potential Motor Goals in PMd

(A and B) Distribution of DMC values and the monkeys' choice-behavior (insets) of the balanced (A) and biased (B) data sets. Same conventions apply as in Figures 3 and $5\left({ }^{* * *} \mathrm{p}<0.001,{ }^{\star *} \mathrm{p}<0.01\right.$, rank-sum test).

(C) The choice-selective analysis of the DMC similarity in PMd. Same conventions apply as in Figure 4.

the neural directional selectivity in the population of PRR neurons. The inferred-goal neural preference is neither consistent with an unbiased equipotent encoding of the two taskdefined motor goal options (options hypothesis), nor with an encoding of the previous instruction cue (visual memory), but it is consistent with the preference hypothesis.

Based on the observed inferred-goal selectivity in the biased data set alone, one could not dissociate preference encoding from preliminary selection encoding. But we can argue against the latter possibility based on the choice-independent bimodal response profiles in the choice-selective analysis of the balanced data set. Preliminary selection encoding would have had to reveal direct-goal neural selectivity in direct-choice trials, and inferred-goal selectivity in inferred-choice trials, which was not the case (see above).

Motor-Goal Encoding in Dorsal Premotor Cortex (PMd) Another objective of our study was to compare parietal and premotor sensorimotor areas, which are well known to be involved in reach planning, while their role in reaching decisions is less clear (Cisek and Kalaska, 2005; Scherberger and Andersen, 2007). We conducted the same analyses for PMd as for PRR neurons. The biased data set contained 193 PMd neurons (118 monkey A, 75 monkey S), and the balanced data set 112 PMd neurons (monkey S). Of those, $46 \%$ fulfilled the criteria for the DMC analysis in the biased data set, and $40 \%$ in the balanced data set, which denote smaller fractions of neurons than in PRR (see above).

The analyses of potential motor-goal encoding in PMd revealed overall very similar results to PRR, but there were also differences. The distribution of DMC values in the biased data set of PMd (Figure 6B) revealed a significant bias in favor of the inferred motor goal $\left(\mathrm{m}=-0.298\right.$; SEM $=0.038, \mathrm{p}<10^{-10}, \mathrm{t}$ test), as it was the case in PRR (Figure 5C, inset). In contrast to PRR (Figure 3C, inset), the DMC distribution in PMd (Figure 6A) also showed a significant remaining bias for inferred goals $(\mathrm{m}=-0.11$; SEM $=0.05, \mathrm{p}=0.004)$ in the balanced data set. Note, though, that this bias in DMC values was significantly smaller $(p=0.002)$ than in the biased data set, which indicates that most neurons exhibited bimodal response profiles, while few had a weak bias for the inferred goal. Since the monkeys also had a small residual choice preference for the inferred goal (Figure $3 \mathrm{~A}$ ) this could mean that PMd is more strongly modulated by small choice preferences than PRR. The choiceselective analyses of the PMG-NC trials showed a high DMC similarity (Figure 6C), equivalent to PRR (Figure 4B). This, like in PRR, indicated that the bimodal directional selectivity was mostly not the consequence of preliminary selection encoding in combination with trial-by-trial switching of the behavioral choice. In summary, the PMd results are qualitatively very similar to PRR, suggesting similar encoding schemes in both areas. For a discussion of additional smaller differences between PRR and PMd as revealed by our model-based analyses and variance analyses see Figures S1 and S2.

Normalization of Multiple Motor-Goal Representations Models of decision making often involve mutual competition between the neural representations of multiple coexisting alternative choices (Platt and Glimcher, 1999; Cisek, 2006). Such competition implies that the response of a neuron should be reduced when its preferred motor goal marks only one out of two equally valid behavioral options, compared to when the motor goal is unambiguously selected. The responses of the example neurons and the population activity plots in Figures 3 and 5 suggest that this is the case. The results indicate a halving of the neural response strength to each potential motor goal in the balanced PMG task compared to the corresponding unambiguous motor goal in the DMG task or biased PMG task. A quantitative analysis of the weight coefficients (scaling factors) in the model-based analysis confirmed this view (Figure S4). The reduced neural response strengths during the simultaneous presence of two alternative motor goals compared to a single 
goal argues in favor of a competition between alternative motor goal representations.

\section{DISCUSSION}

The ability to plan multiple upcoming actions and decide among them is vital to an organism acting within a complex environment. We investigated how parietal and premotor reach planning areas encode the decision between different possible sensorimotor transformation rules that could be applied to a single visuospatial object. When monkeys were faced with two alternative spatial transformations, and chose them with equal preference, then two separate spatial motor goal representations coexisted in the frontoparietal reach network. This was the case despite the fact that only one goal was directly visually cued and the other had to be inferred from the visual cue by applying a remapping rule. Additionally, the parietal reach region (PRR) and the dorsal premotor cortex (PMd) predominantly encoded the variable choice preference between two potential motor goals. By using free-choice probe trials and two distinct reward schedules, we could rule out encoding of the monkeys' preliminary behavioral selections, as well as encoding of the task-defined choice options, during movement planning. Our results suggest that in rule-selection experiments the sensorimotor system first computes all potential motor goals associated with a currently valid set of potential transformation rules, weighs them according to the subject's choice preference, and then selects among these goals.

\section{Deciding among Alternative Action Plans Rather than Transformation Rules or Targets}

We showed that during movement planning two alternative potential reach goals can be represented simultaneously in PRR and PMd in a rule-selection task. In this task only one visuospatial target was presented at a time, allowing two alternative motor goals by applying two different mapping rules. Our results suggest that with preexisting knowledge about the visuospatial constraints of the task (knowing the spatial cue), and uncertainty about the to-be-applied rule (not knowing the context cue), the sensorimotor system constructs all remaining motor goal options, which are defined by the general context of the task, and are of subjective value to the monkey (see biased versus balanced condition below). We can reject the alternative ruleselection hypothesis according to which the monkeys in general would first select a rule, and then only compute the single associated motor plan. It is as if the sensorimotor system in a rule-selection task first creates all potential motor-goal representations and then applies the same computational decision algorithms as in a target-selection task.

The view that multiple spatial motor goal options can be simultaneously encoded prior to the decision in parietal and premotor areas is reminiscent of earlier saccadic target-selection experiments in the superior colliculus (Basso and Wurtz, 1998) and the lateral intraparietal area LIP (Platt and Glimcher, 1999; Sugrue et al., 2004; Dorris and Glimcher, 2004; Yang and Shadlen, 2007; Louie and Glimcher, 2010). They showed probabilistic, graded neural responses for preferred and nonpreferred targets, depending on saccadic choice probabilities or subjective values.
Also, a study in PMd showed bimodal response profiles in a manual two-target selection task (Cisek and Kalaska, 2005). Our conclusions go beyond the previous findings, since these studies showed the coexistence of multiple spatial representations associated with alternative choices, but used target-selection tasks. We show that the simultaneous representation of mutually exclusive spatial motor goals in sensorimotor areas does not require the presentation of multiple alternative spatial physical target stimuli.

This simultaneous encoding of alternative competing motor goals is also fundamentally different from the representation of two sequential movement goals. Previous experiments showed that in the parietal cortex, during the planning of a multicomponent (double-step) movement, two neural populations were activated, each of which was selective for one of the single movement components (Medendorp et al., 2006; Baldauf et al., 2008). Double-step experiments do not induce a decision process between mutually exclusive action goals, and rather suggest that multiple components of a complex movement can be planned at once. Our finding of simultaneous encoding of alternative competing motor goals does complement previous observations in effector-selection experiments, which showed that alternative eye or hand movements to the same spatial target, instructed (Calton et al., 2002) or freely chosen (Cui and Andersen, 2007), can elicit simultaneous movement planning activity in LIP and PRR.

The advantage of the goal-selection scheme over the ruleselection scheme for decision making could be that-by computing all associated motor goal alternatives and their implicit action plans during the ambiguous state of planning-a more comprehensive cost-benefit calculation of each choice can be achieved. When the striker in our introductory example has to decide between aiming for the position of the goal keeper versus the opposite corner, then it is not enough to consider the likelihood of the keeper to jump or stay. Also the costs associated with the striker's action alternatives are relevant, e.g., the striker might be poor at aiming for right-side goals, or the ball might be in an immediate position that eases aiming for one corner but not the other. Our results imply that the decision process in our rule-selection experiment selected between competing motor-goal alternatives, not between different transformation rules or target stimuli, and that this competition likely happened in the sensorimotor areas that are involved in planning the respective movements.

Note, we do note rule out the possibility that in parallel a competition between the two potential rules takes place in rule-encoding frontal cortical areas (White and Wise, 1999; Wallis et al., 2001; Wallis and Miller, 2003; Genovesio et al., 2005). The rule-competition could then, in the extreme case, just be mirrored by probabilistic motor goal representations in downstream sensorimotor areas. Because of the observed response normalization in our data (see below), we believe that if at all there was a rule-competition in our task then it was paralleled by a goal-competition in the sensorimotor areas, which would make sense for economical reasons, as discussed in the previous paragraph (Cisek and Kalaska, 2010).

\section{Preference versus Options Encoding}

Potential motor-goal representations in our experiment depended on the preference of the monkeys, as defined by the 
probability of behavioral choice of either action alternative. In our task design we cannot differentiate between choice probabilities and assigned subjective value (Sugrue et al., 2004; Samejima et al., 2005; Hampton et al., 2006; Kable and Glimcher, 2007; Lau and Glimcher, 2008; Wunderlich et al., 2009), as was attempted in a recent discounting experiment (Louie and Glimcher, 2010). Consequently, we speak more generally of preferences, as quantified by choice probabilities.

Simultaneous potential motor-goal encoding during reach planning had previously only been shown in PMd (Cisek and Kalaska, 2005). Since a dependence on the monkeys' choice preferences was not tested, it is unclear if this previous PMd data reflected preferences or task-defined motor-goal options (the menu, Padoa-Schioppa and Assad, 2006). The biased population tuning in the memory period of our biased data set contradicts options encoding, and suggests that potential motor-goal encoding predominantly reflected choice preferences in PMd.

In posterior parietal cortex, preference encoding between competing options has previously been shown in saccadic target-selection tasks (Platt and Glimcher, 1999; Sugrue et al., 2004; Dorris and Glimcher, 2004; Yang and Shadlen, 2007; Kable and Glimcher, 2007; Wunderlich et al., 2009; Louie and Glimcher, 2010). Corresponding data for skeletomotor movements, like reaching, and for rule-selection tasks in general is lacking. Previous target-selection tasks with reaching revealed post-GO-cue selection signals in PRR (Scherberger and Andersen, 2007; Pesaran et al., 2008), but no neural response modulations by choice preference was shown. Previous tasks with deterministic targets showed reward- or value-dependent modulations of the neural responses (Musallam et al., 2004; lyer et al., 2010), but relative weighing of alternative options against each other was not tested.

Taken together, the principle of weighing alternative motor goal representations with behavioral choice preferences is not restricted to the saccade planning system, but can be found in the skeletomotor system as well, and neural implementations of this principle include not only parietal movement planning areas, but also areas in the frontal cortex, like PMd.

\section{Competing Goal Representations}

Models of decision making often imply mutual competition between the neural representations of multiple coexisting alternative choices (Platt and Glimcher, 1999; Cisek, 2006). In our experiment, this competition likely happened in the sensorimotor areas that we recorded from and that are involved in planning the respective movements, since we found reduced neural response strengths during the simultaneous representation of two alternative motor goals compared to a single goal (Cisek and Kalaska, 2005).

\section{Conclusions}

Our findings support the idea that reach decision making and movement planning, in tasks that require the selection of a spatial transformation rule, are integrative rather than sequential processes, mediated by overlapping action-specific neural populations in PRR and PMd (Scherberger and Andersen, 2007; Cisek, 2007; Andersen and Cui, 2009). The results provide evidence for competitive encoding of alternative potential reach plans in PRR and PMd, reflecting the monkeys' average choice preferences, but being independent of the immediate behavioral choice of the monkey. This is consistent with the idea that the brain utilizes probabilistic representations throughout all stages of the decision process until an action is finally required (Knill and Pouget, 2004). Importantly, our results suggest that in situations of uncertain choice of which transformation rule to apply, the sensorimotor system can construct all potential motor goal alternatives, and then select among these alternatives, once enough evidence for a proper choice is available, rather than preliminarily betting on one of the transformation rules and computing only the single corresponding motor plan. This strategy could denote a valuable and general principle in decision making, allowing a more comprehensive cost-benefit analysis that includes the consequential costs of the movements associated with each choice.

\section{EXPERIMENTAL PROCEDURES}

Potential Motor Goal Task with Context Instruction In PMG-Cl trials (Figure 2), one spatial and one contextual visual cue were presented to the subjects at different times during the trial (ViewSonic VX922 LCD screen; $5 \mathrm{~ms}$ off-on-off response time). The peripheral spatial cue was located at one of four possible positions $\left(0^{\circ}, 90^{\circ}, 180^{\circ}\right.$, and $\left.270^{\circ}\right)$ with an eccentricity of $9 \mathrm{~cm}\left(14.5^{\circ}\right.$ visual angle, VA) relative to the fixation point. The contextual cue consisted of a green (direct-cued) or blue (inferred-cued) frame around the central eye and hand fixation points. It instructed the subject to reach toward (direct, proreach) or to the position diametrically opposite of the spatial cue (inferred, antireach).

A trial was initiated by the monkey by fixating a small red square in the center of the screen (eye fixation tolerance: 2.0-3.0 VA; $224 \mathrm{~Hz}$ CCD camera, ET-49B, Thomas Recording, Giessen, Germany) and touching an adjacent white square of the same size (hand fixation tolerance: $4.0^{\circ} \mathrm{VA}$, touch screen mounted directly in front of the video screen; IntelliTouch, ELO Systems, Menlo Park, CA). After a random period of 500-1000 ms (fixation period) the spatial cue was shown briefly for 200 ms. During the following 800-2000 ms (memory period) only the fixation squares were visible. The contextual cue was shown for $170 \mathrm{~ms}$ at the end of the memory period and the hand fixation square disappeared (GO signal). The monkey had to make a reach toward the instructed goal within a maximum of $700-1000 \mathrm{~ms}$ (movement period, $4.9^{\circ} \mathrm{VA}$ reach tolerance) and hold the goal position for $300-400 \mathrm{~ms}$ (feedback period). The monkey received visual feedback about the correct movement goal (filled circle of the same color as the contextual cue at the goal location) at the end of a correct trial.

Eye fixation had to be kept throughout the trial. Liquid reward and acoustic feedback indicated correct (high pitch tone, reward) or incorrect (low pitch tone, no reward) behavior. Correct choice of the instructed motor goal and fixation behavior were required for a PMG-Cl trial to be considered correct. Only correct trials were used for the analysis.

Potential Motor Goal Task without Context Information

PMG-NC trials were similar to the PMG-Cl trials, except that no contextual cue was shown at the end of the memory period. In those trials the monkey had to choose whether to reach to the direct or to the inferred goal. Until the end of the memory period PMG-Cl and PMG-NC trials were indistinguishable. Only PMG-NC trials in which the monkey either reached for the direct or the inferred position were considered correct and were used for the analysis. Note that not all of the correct trials were rewarded. Reward depended on the used reward schedule (see below).

\section{Definite Motor Goal Task}

The DMG task differed from the PMG-Cl trials only in the timing of the contextual cue. In the DMG task the spatial and the contextual cue were shown simultaneously at the beginning of the memory period. Only DMG trials with correct choices and ocular fixation were rewarded and analyzed. 
Block Design and Randomization

The PMG and DMG tasks were presented in separate blocks. The DMG block consisted of typically $\sim 100$ trials, the PMG block of a minimum of $\sim 300$ trials. The order of the two tasks was variable across days.

PMG-NC and PMG-Cl trials were randomly interleaved during PMG blocks. A PMG block contained 60\%-80\% (mean $=76 \%$ ) PMG-Cl trials and $20 \%-40 \%$ (mean $=24 \%$ ) PMG-NC trials. In each task the four spatial cuing directions were randomly interleaved with equal probability. In PMG-Cl trials and in the DMG task the direct-cued and inferred-cued trials were also randomly interleaved with equal probability.

\section{Reward Schedules in PMG-NC Trials}

We implemented two different reward schedules for PMG-NC trials.

One was the bias-minimizing reward schedule (BMRS). With a BMRS balanced behavior, i.e., $50 \%$ direct and $50 \%$ inferred reaches, leads to a $50 \%$ reward probability, while any biased choice behavior leads to lower reward probabilities. The BMRS algorithm takes the reward history of the monkeys into account and changes the probabilities for rewarding a direct or inferred reach in favor of the alternative that was chosen less often so far:

$$
\begin{aligned}
& p\left(R_{d}\right)=F\left(n_{i}-n_{d}\right), \\
& p\left(R_{i}\right)=F\left(n_{d}-n_{i}\right)
\end{aligned}
$$

where $n_{i}$ is the total number of rewarded inferred reaches and $n_{d}$ is the total number of rewarded direct reaches. $F$ was defined as

$$
F(x):=\left\{\begin{array}{ll}
1 & x>1 \\
2 / 3 & x=1 \\
1 / 2 & x=0 \\
1 / 3 & x=-1 \\
0 & x<-1
\end{array} .\right.
$$

The second reward schedule was the equal-probability reward schedule (EPRS). In EPRS trials the monkeys were rewarded with 50\% probability, no matter whether they reached for the direct or inferred goal, and regardless of the reward history. The reward probabilities for direct $\left(R_{d}\right)$ or inferred $\left(R_{i}\right)$ choices were

$$
p\left(R_{d}\right)=p\left(R_{i}\right)=0.5 .
$$

With the EPRS, the reward probability is independent of the behavioral strategy of the monkeys, as long as they chose between the two potential goals (see Figure S5 for data with 100\% reward probability).

\section{Biased and Balanced Condition}

The recorded data was split into two distinct data sets. One data set contains only units that were recorded with the EPRS, before we trained the respective monkey with the BMRS. Since both monkeys showed a very similar choice bias during EPRS sessions (see Results), we refer to this data as the biased data set. The second data set contains only units recorded after we used BMRS and is referred to as balanced data set. Behavioral tests with the PMG-NC trials were conducted at the end of the neuronal recording period in the biased data set. Control experiments with simultaneous behavioral and neural recording of biased PMG-NC trials confirmed that results and conclusions are unaffected by this (see Figure S5).

\section{Animal Preparation, Neural Recordings}

Surgical procedures and neural recordings were described previously (Gail et al., 2009). Animal care and all experimental procedures were conducted in accordance with German laws governing animal care.

\section{Neural Data Selection and Tuning Analyses}

Extracellular recordings were conducted with up to five microelectrodes in parallel ("mini-matrix"; Thomas Recording, Giessen, Germany) on each chamber. Spike times and waveforms were recorded and subjected to additional offline sorting (Offline Sorter; Plexon).

All isolated units were tested for their directional selectivity (Kruskal-Wallis test; four groups of different spatial cue positions; sample sizes defined by the number of identical trial repetitions). Selectivity was tested independently for direct-cued and inferred-cued trials during the late memory period in the DMG task (average spike rate during the last $300 \mathrm{~ms}$ of the memory period, i.e., activity succeeding the precue with a time-lag of at least $500 \mathrm{~ms}$, and immediately preceding the GO cue). The late memory period was chosen to extract movement planning activity without confounding effects of (1) immediate visual input from the cue stimuli; (2) transition phases from visual to motor-goal tuning (Gail and Andersen, 2006); or (3) visual and somatosensory input and motor-control signals related to movement initiation.

Only neurons that were significantly selective in direct-cued trials of the DMG task were used in the following analyses (Figure S6). For all analyses that involved PMG-Cl or PMG-NC trials, we additionally required the neurons to be significantly directionally selective in the late memory period of PMG trials (Kruskal-Wallis, see above).

\section{Population Activity}

To visualize the temporal dynamics of spatial representations on a population level, we averaged the time-resolved spiking activity across all neurons that were directionally selective during the memory period of PMG trials. Before averaging, the directional selectivity profiles for each neuron were aligned relative to the interpolated preferred direction in the late memory period of the DMG task and normalized to the baseline level (average spike density in the $300 \mathrm{~ms}$ before spatial cue onset). The population activity was only used for illustrative purposes (see Figures $3 \mathrm{C}$ and $5 \mathrm{C}$ ), not for quantitative statistical analyses.

\section{Analysis of Bimodal Selectivity Profiles}

We used a direction modality contrast (DMC) to quantify the bimodality of individual neuronal responses:

$$
D M C=\frac{R_{M D}-R_{O D}}{R_{M D}+R_{O D}} .
$$

$R_{M D}$ is the mean firing rate of a neuron during the last 300 ms of the memory period of all PMG trials (PMG-Cl and PMG-NC) at the same direction that evoked the maximum response (MD) in the DMG task. $R_{O D}$ is the firing rate for trials in the opposite-to-maximum direction (OD). Since the MD is measured relative to the direction of the spatial cue in direct-cued trials of the DMG task, positive DMC indices indicate preferred selectivity for the direct motor goal (at the spatial cue location), whereas negative values indicate preferred selectivity for the inferred motor goal (opposite the spatial cue). Values around zero indicate symmetric bimodal selectivity, not lack of selectivity, since neurons without directional selectivity were removed from this analysis.

\section{Choice-Selective Analyses}

To differentiate between the selection and the preference hypotheses, we sorted the PMG-NC trials in the balanced data set according to the free choice of the monkey, and calculated the DMC separately for direct-choice and inferred-choice trials. That means if in a PMG-NC task the monkey reached toward a goal position as if the contextual instruction had been direct, the trial was labeled "direct choice" and if he reached toward a goal position as if the contextual instruction had been inferred, the trial was labeled "inferred choice." The absolute choice-selective DMC values were then compared to the absolute original, choice-indifferent DMC values (average over all trials without sorting them according to the choice) in a similarity analysis (illustrated in Figure 4A). The DMG condition was used as a control for this similarity analysis (see Figure S2). To quantify the similarity between the choice-selective DMC values and the choice-indifferent DMC values, we calculated the distance from the unity line of the correlation plot, which is equivalent of calculating the difference between the choice-selective and choice-indifferent DMC values. We then used a $t$ test to determine if the distribution of these differences was significantly deviating from zero.

\section{SUPPLEMENTAL INFORMATION}

Supplemental Information includes six figures and can be found with this article online at doi:10.1016/j.neuron.2011.02.053. 


\section{ACKNOWLEDGMENTS}

We thank Sina Plümer and Ludwig Ehrenreich for their help in data collection. This work was supported by the Federal Ministry for Education and Research (BMBF, Germany, grants 01GQ0433, 01GQ0814, 01GQ1005C), the Deutsche Forschungsgemeinschaft (DFG) Collaborative Research Centre 889 "Cellular Mechanisms of Sensory Processing," and the Alexander von Humboldt Foundation.

Accepted: February 23, 2011

Published: May 11, 2011

\section{REFERENCES}

Andersen, R.A., and Cui, H. (2009). Intention, action planning, and decision making in parietal-frontal circuits. Neuron 63, 568-583.

Baldauf, D., Cui, H., and Andersen, R.A. (2008). The posterior parietal cortex encodes in parallel both goals for double-reach sequences. J. Neurosci. 28 , 10081-10089.

Basso, M.A., and Wurtz, R.H. (1998). Modulation of neuronal activity in superior colliculus by changes in target probability. J. Neurosci. 18, 7519-7534.

Batista, A.P., Buneo, C.A., Snyder, L.H., and Andersen, R.A. (1999). Reach plans in eye-centered coordinates. Science 285, 257-260.

Bisley, J.W., and Goldberg, M.E. (2010). Attention, intention, and priority in the parietal lobe. Annu. Rev. Neurosci. 33, 1-21.

Calton, J.L., Dickinson, A.R., and Snyder, L.H. (2002). Non-spatial, motorspecific activation in posterior parietal cortex. Nat. Neurosci. 5, 580-588.

Churchland, A.K., Kiani, R., and Shadlen, M.N. (2008). Decision-making with multiple alternatives. Nat. Neurosci. 11, 693-702.

Cisek, P. (2006). Integrated neural processes for defining potential actions and deciding between them: A computational model. J. Neurosci. 26, 9761-9770.

Cisek, P. (2007). Cortical mechanisms of action selection: The affordance competition hypothesis. Philos. Trans. R Soc. Lond. B Biol. Sci. 362, 15851599.

Cisek, P., and Kalaska, J.F. (2005). Neural correlates of reaching decisions in dorsal premotor cortex: Specification of multiple direction choices and final selection of action. Neuron 45, 801-814.

Cisek, P., and Kalaska, J.F. (2010). Neural mechanisms for interacting with a world full of action choices. Annu. Rev. Neurosci. 33, 269-298.

Crammond, D.J., and Kalaska, J.F. (1994). Modulation of preparatory neuronal activity in dorsal premotor cortex due to stimulus-response compatibility. J. Neurophysiol. 71, 1281-1284.

Crammond, D.J., and Kalaska, J.F. (2000). Prior information in motor and premotor cortex: Activity during the delay period and effect on pre-movement activity. J. Neurophysiol. 84, 986-1005.

Cui, H., and Andersen, R.A. (2007). Posterior parietal cortex encodes autonomously selected motor plans. Neuron 56, 552-559.

Dorris, M.C., and Glimcher, P.W. (2004). Activity in posterior parietal cortex is correlated with the relative subjective desirability of action. Neuron 44 , 365-378.

Dorris, M.C., and Munoz, D.P. (1998). Saccadic probability influences motor preparation signals and time to saccadic initiation. J. Neurosci. 18, 7015-7026.

Everling, S., Dorris, M.C., Klein, R.M., and Munoz, D.P. (1999). Role of primate superior colliculus in preparation and execution of anti-saccades and prosaccades. J. Neurosci. 19, 2740-2754.

Gail, A., and Andersen, R.A. (2006). Neural dynamics in monkey parietal reach region reflect context-specific sensorimotor transformations. J. Neurosci. 26, 9376-9384.

Gail, A., Klaes, C., and Westendorff, S. (2009). Implementation of spatial transformation rules for goal-directed reaching via gain modulation in monkey parietal and premotor cortex. J. Neurosci. 29, 9490-9499.

Genovesio, A., Brasted, P.J., Mitz, A.R., and Wise, S.P. (2005). Prefrontal cortex activity related to abstract response strategies. Neuron 47, 307-320.
Gold, J.I., and Shadlen, M.N. (2007). The neural basis of decision making. Annu. Rev. Neurosci. 30, 535-574.

Hampton, A.N., Bossaerts, P., and O'Doherty, J.P. (2006). The role of the ventromedial prefrontal cortex in abstract state-based inference during decision making in humans. J. Neurosci. 26, 8360-8367.

Iyer, A., Lindner, A., Kagan, I., and Andersen, R.A. (2010). Motor preparatory activity in posterior parietal cortex is modulated by subjective absolute value. PLoS Biol. 8, e1000444.

Kable, J.W., and Glimcher, P.W. (2007). The neural correlates of subjective value during intertemporal choice. Nat. Neurosci. 10, 1625-1633.

Kable, J.W., and Glimcher, P.W. (2009). The neurobiology of decision: Consensus and controversy. Neuron 63, 733-745.

Kim, B., and Basso, M.A. (2010). A probabilistic strategy for understanding action selection. J. Neurosci. 30, 2340-2355

Knill, D.C., and Pouget, A. (2004). The Bayesian brain: The role of uncertainty in neural coding and computation. Trends Neurosci. 27, 712-719.

Lau, B., and Glimcher, P.W. (2008). Value representations in the primate striatum during matching behavior. Neuron 58, 451-463.

Louie, K., and Glimcher, P.W. (2010). Separating value from choice: Delay discounting activity in the lateral intraparietal area. J. Neurosci. 30, 5498-5507.

Medendorp, W.P., Goltz, H.C., and Vilis, T. (2005). Remapping the remembered target location for anti-saccades in human posterior parietal cortex. J. Neurophysiol. 94, 734-740.

Medendorp, W.P., Goltz, H.C., and Vilis, T. (2006). Directional selectivity of BOLD activity in human posterior parietal cortex for memory-guided doublestep saccades. J. Neurophysiol. 95, 1645-1655.

Mountcastle, V.B., Lynch, J.C., Georgopoulos, A.P., Sakata, H., and Acuna, C. (1975). Posterior parietal association cortex of the monkey: Command functions for operations within extrapersonal space. J. Neurophysiol. 38, 871-908.

Musallam, S., Corneil, B.D., Greger, B., Scherberger, H., and Andersen, R.A. (2004). Cognitive control signals for neural prosthetics. Science 305, 258-262.

Padoa-Schioppa, C., and Assad, J.A. (2006). Neurons in the orbitofrontal cortex encode economic value. Nature 441, 223-226.

Pesaran, B., Nelson, M.J., and Andersen, R.A. (2008). Free choice activates a decision circuit between frontal and parietal cortex. Nature 453, 406-409.

Platt, M.L., and Glimcher, P.W. (1999). Neural correlates of decision variables in parietal cortex. Nature 400, 233-238.

Rangel, A., Camerer, C.F., and Montague, P.R. (2008). A framework for studying the neurobiology of value-based decision making. Nat. Rev. Neurosci. 9, 545-556.

Samejima, K., Ueda, Y., Doya, K., and Kimura, M. (2005). Representation of action-specific reward values in the striatum. Science 310, 1337-1340.

Scherberger, H., and Andersen, R.A. (2007). Target selection signals for arm reaching in the posterior parietal cortex. J. Neurosci. 27, 2001-2012.

Schlag-Rey, M., Amador, N., Sanchez, H., and Schlag, J. (1997). Antisaccade performance predicted by neuronal activity in the supplementary eye field. Nature 390, 398-401.

Shen, L., and Alexander, G.E. (1997). Preferential representation of instructed target location versus limb trajectory in dorsal premotor area. J. Neurophysiol. 77, 1195-1212.

Snyder, L.H., Batista, A.P., and Andersen, R.A. (1997). Coding of intention in the posterior parietal cortex. Nature 386, 167-170.

Sugrue, L.P., Corrado, G.S., and Newsome, W.T. (2005). Choosing the greater of two goods: Neural currencies for valuation and decision making. Nat. Rev. Neurosci. 6, 363-375.

Sugrue, L.P., Corrado, G.S., and Newsome, W.T. (2004). Matching behavior and the representation of value in the parietal cortex. Science 304, 1782-1787.

Tremblay, L., Gettner, S.N., and Olson, C.R. (2002). Neurons with objectcentered spatial selectivity in Macaque SEF: Do they represent locations or rules? J. Neurophysiol. 87, 333-350. 
Wallis, J.D., and Miller, E.K. (2003). From rule to response: Neuronal processes in the premotor and prefrontal cortex. J. Neurophysiol. 90, 1790-1806.

Wallis, J.D., Anderson, K.C., and Miller, E.K. (2001). Single neurons in prefrontal cortex encode abstract rules. Nature 411, 953-956.

Weinrich, M., and Wise, S.P. (1982). The premotor cortex of the monkey. J. Neurosci. 2, 1329-1345.

White, I.M., and Wise, S.P. (1999). Rule-dependent neuronal activity in the prefrontal cortex. Exp. Brain Res. 126, 315-335.

Wise, S.P., di Pellegrino, G., and Boussaoud, D. (1996). The premotor cortex and nonstandard sensorimotor mapping. Can. J. Physiol. Pharmacol. 74, 469-482.
Wunderlich, K., Rangel, A., and O'Doherty, J.P. (2009). Neural computations underlying action-based decision making in the human brain. Proc. Natl. Acad. Sci. USA 106, 17199-17204.

Yanai, Y., Adamit, N., Israel, Z., Harel, R., and Prut, Y. (2008). Coordinate transformation is first completed downstream of primary motor cortex. J. Neurosci. 28, 1728-1732.

Yang, T., and Shadlen, M.N. (2007). Probabilistic reasoning by neurons. Nature 447, 1075-1080.

Zhang, M., and Barash, S. (2004). Persistent LIP activity in memory antisaccades: Working memory for a sensorimotor transformation. J. Neurophysiol. 91, 1424-1441. 


\section{Supplemental information S1 - Model-based tuning analysis (related to Figures} 3-6)

The analysis of the direction modality contrast (DMC) only addresses the spike rate difference between the direction with the maximum response (MD) and the opposite direction (OD) for each neuron. Other characteristics of spatial selectivity are not considered, like the exact preferred direction, which can lie between the probed directions, and gain-modulation effects between direct and inferred reaches, which have been shown to occur in both PRR and PMd (Gail et al. 2009). For example, a neuron which is tuned for the same reach direction in direct-cued and inferred-cued trials, but due to gain-modulation has a substantially different overall spike rate in the two contexts (e.g. $15 \mathrm{~Hz}$ in the direct context and $30 \mathrm{~Hz}$ in the inferred context), could show an asymmetric bimodal selectivity profile in the balanced PMG data. The DMC index in this case would be negative, indistinguishable from the DMC of a unimodal selectivity profile, and erroneously suggesting the representation of only a single spatial motor goal at the inferred position.

Therefore, as an alternative to the DMC, we modeled the to-be-expected selectivity profile in the PMG task $\left(T^{P M G}\right)$ as a linear combination of the selectivity profiles in the direct-cued $(d)$ and inferred-cued $(i)$ DMG trials:

$$
T^{P M G}=A\left(\alpha_{d} T_{d}^{D M G}+\alpha_{i} T_{i}^{D M G}\right)
$$

Each selectivity profile $T$ consists of a 4-element vector with the neuron's responses to the four probed cue locations (left, right, up, down). With the model profiles we tested three discrete alternative coding hypotheses. We fixed the linear coefficients 
and $\alpha_{i}$ to different preset values for each hypothesis and fitted $A$ as the only free parameter on a least square basis. When both motor goals are encoded with equal strength, then the tuning in the PMG task should combine direct and inferred tuning of the DMG task with equal weights ( $\alpha_{d}=\alpha_{I}=0.5$; mean model). When a unique goal is encoded, then the PMG selectivity profile should either reflect the DMG selectivity profile of direct reach trials $\left(\alpha_{d}=1, \alpha_{l}=0\right.$; direct model), or the inferred reach trials ( $\alpha_{d}=0, \alpha_{i}=1$; inferred model), depending on which of these two alternatives was preferred or selected. We predicted the PMG selectivity profiles from the DMG profiles for these three models, and compared the mean square error (MSE) between the predicted and the measured profiles. We computed spike densities (Szucs, 1998) for a time-continuous representation of the neural response by convolving each spike train with a Gaussian kernel $(\sigma=50 \mathrm{~ms})$ and averaging across trials. We then determined the best-fitting model for each neuron as a function of time. In each time bin the three models were fitted to the tuning profiles, separately in the PMG-CI and PMG-NC task. In the PMG-CI task the trials were sorted according to direct- and inferred-goal instruction. In the PMG-NC task, trials were sorted according to directand inferred-goal choice of the monkeys.

To quantify preference and selection encoding we defined a 'preference signal' and a 'selection signal' (Fig. S1b bottom). The preference signal was determined by the total number of neurons in the PMG-NC task of the balanced dataset, which best fit the mean model, irrespective of the choice of the monkey. That means if a neuron fits best with the mean model in direct- and inferred-choice trials for a particular point in time it contributed to the preference signal for that point in time. The selection signal was likewise determined by the total number of neurons in the PMG-NC task 
of the balanced dataset, which best fit the direct model in direct-choice trials and the inferred model in inferred-choice trials.

Note that in the instructed PMG-CI trials we cannot determine the selection encoding during the memory period, since any preliminary selection signals would have to be overruled, and hence obscured, by the context instruction at the time of the 'GO'-cue. In non-instructed PMG-NC trials, on the other hand, preliminary selection signals could be detectable prior to the 'GO'-signal and would be predictive about the pending choice. This is why we only computed the preference and selection signals for the PMG-NC trials. After the 'GO'-signal we expect the selection signal to become dominant irrespective of the strength of the signal in the previous memory period, because the situation then is similar to a DMG task.

Note also that in the biased dataset the preference and selection signal can not be distinguished since the strong behavioral bias for inferred goals makes direct-goal choices extremely rare. This is why we analyzed the preference and selection signals only in the balanced dataset.

\section{Model-based PRR results}

In the PMG-CI task, most PRR neurons in the balanced condition (Fig. S1a; top left) were best fitted with the mean model $(66.0 \%)$, while inferred model $(26.8 \%)$ and direct model $(7.2 \%)$ were less prominent during the late memory period. In the biased dataset, the inferred model provided the best fit for most neurons $(84.5 \%)$, followed by the mean $(12.8 \%)$ and direct $(2.7 \%)$ models (Fig. S1a; bottom left). In Figure S1b (left) PMG-NC trials were analyzed accordingly. Dominance of the mean model in the balanced dataset and dominance of the inferred model in the biased dataset 
together suggest predominant encoding of motor-goal preferences in PRR during movement planning.

In the balanced dataset preference and options encoding can not be distinguished. Similarly, we can not distinguish preference and selection encoding in the biased dataset. Our conclusion that the neural populations in our experiment mainly encode preferences are derived from the fact that the unimodal selectivity (inferred model) in the biased dataset rules out general options encoding, and the predominant bimodal selectivity in the balanced dataset (mean model) rules out general selection encoding. The latter dissociation can be best made with a choiceselective analysis of the PMG-NC trials. If the selection hypothesis was true, then the mean model (red curves) would always have to be less likely than the direct model in the trials with direct choices (green solid curve) or the inferred model in trials with inferred choices (blue dotted curve). The two curves at the bottom of the panels in Fig. S1b denote the fraction of neurons at each point in time which, according to the neuron's model fit, complied best with the preference or with the selection hypothesis. For the late memory period (shaded area) the percentage of neurons showing a selection signal was $2.9 \%$. For the neurons to be considered they had to best fit the direct model in direct-choice trials and the inferred model in the inferred-choice trials. The percentage of neurons that showed a preference signal in the same time window was $36.8 \%$. In this case to be considered the neurons had to best fit the mean model in direct- and inferred-choice trials. The preference signal was higher than the selection signal during the memory period, while selection encoding became dominant after the 'GO'-cue.

The fact that the direct model more often than the inferred model was the best fitting model in direct-choice trials, and the inferred model more often was the best 
model in inferred-choice trials, points to partial selection encoding. Yet, in hardly any neuron both conditions were simultaneously met, leading to the negligible fraction of truly selection encoding neurons during the memory period.

\section{Model-based PMd results}

PMd neurons in the balanced condition (Fig. S1a; top right) show a qualitatively similar result as those in PRR (mean model: $51.1 \%$; inferred model: $40.0 \%$; direct model: $8.9 \%$ ), when PMG-CI trials are considered. The same is true for the biased condition (inferred model: $67.4 \%$; mean model: $31.5 \%$; direct model: $1.1 \%$ ). Overall, the results suggest a predominant encoding of motor-goal preferences, like in PRR. Quantitatively, the difference in the fractions of neurons for which the mean or the inferred model provided the best fits was smaller in PMd than PRR. Also, in the PMG-NC trials (Fig. S1b, right), the monkeys' preliminary selections modulated a larger fraction of neurons in PMd, as can be seen from the stronger separation of the direct- (solid) and inferred-choice (dotted) curves and a reduced preference signal (21.1\%) compared to PRR. The percentage of neurons showing a selection signal during the late memory period was $7.6 \%$, which is more than double the amount compared to PRR. We did not observe such large differences between PMd and PRR in the similarity analyses presented in the main text (cp. Fig. 4b and Fig. 6c). Assuming that PMd neurons are only slightly more modulated by a preliminary selection than PRR neurons the different results could be the consequence of the analysis method. The model based analysis classifies neurons by utilizing a winnertake-all mechanism, e.g. even if a model fit is only slightly better than another fit the assignment to a class is absolute. Therefore small modulatory differences between 
Potential motor goal

PMd and PRR can result in large differences in the number of neurons assigned to a particular class. The similarity analysis, on the other hand, does not enhance small differences, which explains why we cannot observe a similar difference between PMd and PRR in the similarity analyses presented in the main text (cp. Fig. 4 and Fig. 6c). 
a

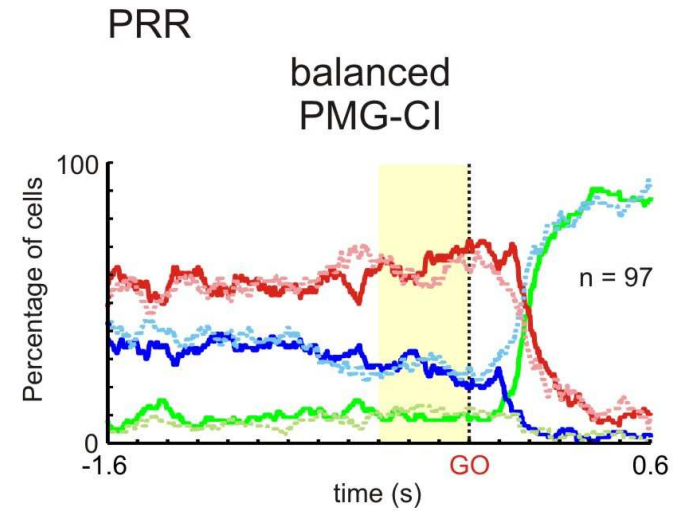

biased

PMG-Cl
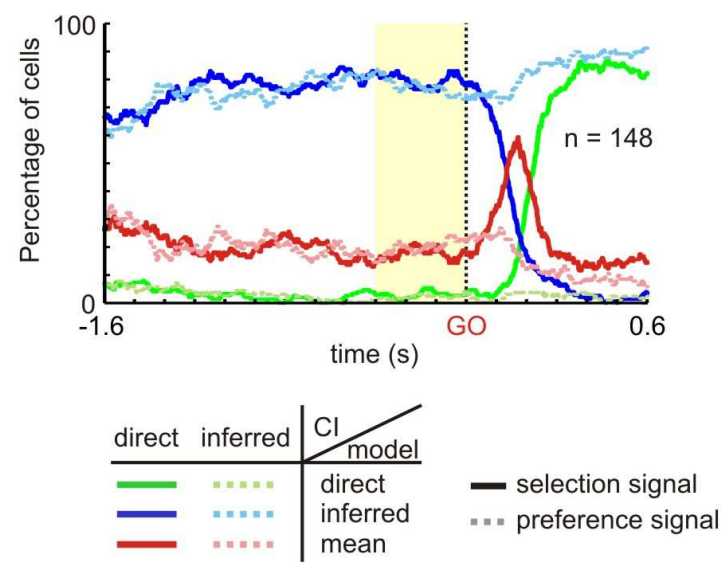

b

PRR

balanced

PMG-NC

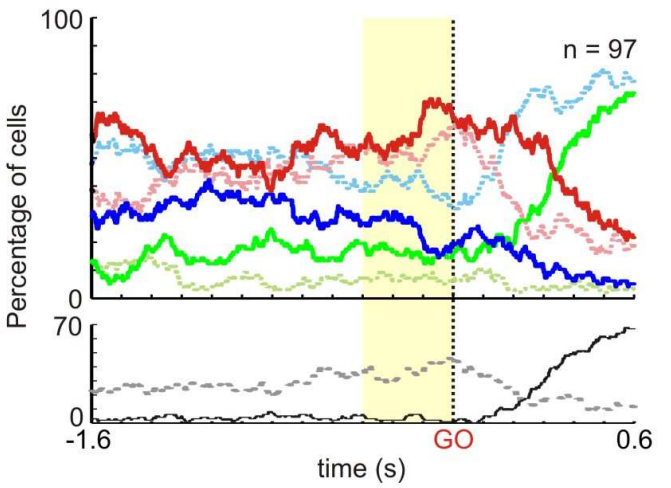

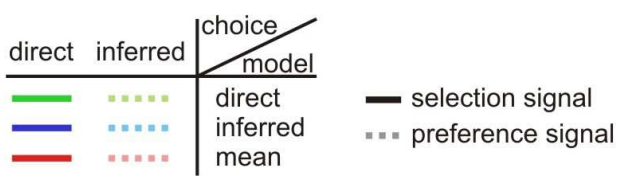

PMd

balanced

PMG-Cl

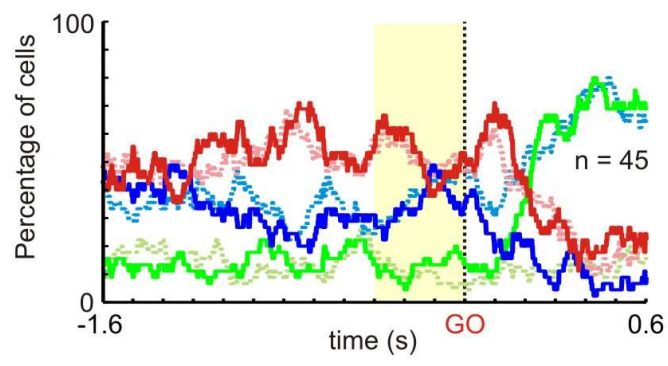

biased

PMG-Cl

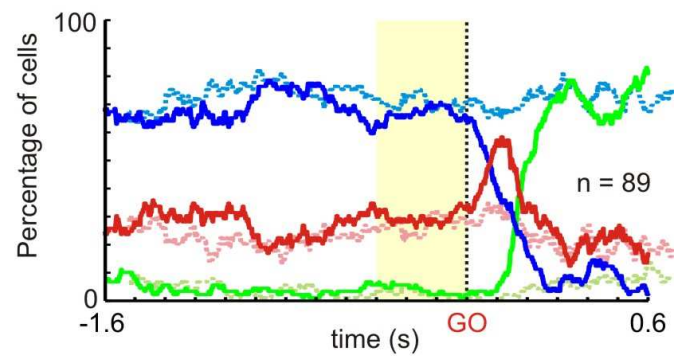

PMd

balanced

PMG-NC

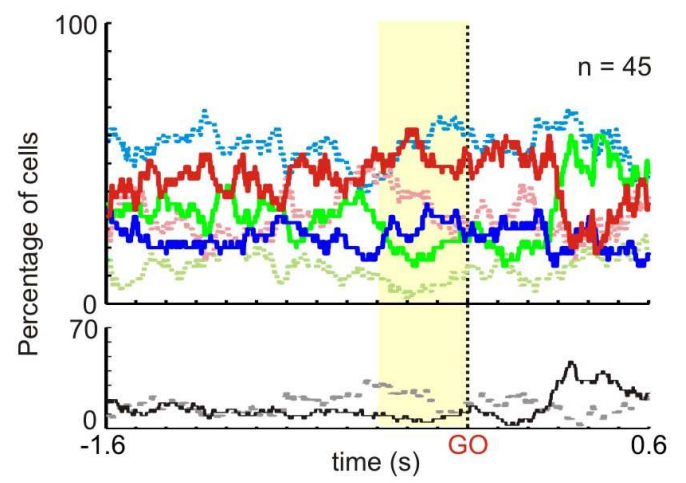

Fig. S1: Results of the model-based analysis for PRR (left) and PMd (right). (a)

Model fits for the balanced (top) and biased (bottom) condition in the PMG-CI task. 
Curves depict the percentage of neurons which fitted best with the direct model (green), inferred model (blue), or mean model (red). Direct-instructed (solid) and inferred-instructed (dotted) trials are analyzed separately. The spike densities were aligned to the 'GO'-signal. The late memory period (shaded area) was used to calculate the mean percentages of neurons listed in the text (SI 1). (b) Same analysis for the PMG-NC task. The smaller graphs (bottom) show the selection (solid black) and preference (dotted grey) signals, as defined in the text. 


\section{Supplemental Information S2 - Control analyses for ruling out the selection hypothesis (related to figures $4 \mathrm{~b}$ and $6 c$ )}

\section{Sensitivity of choice-selective DMC similarity analysis}

The DMC similarity analysis was used to determine if the monkeys used a trial-totrial guess-and-switch behavioral strategy (4b and 6c), i.e. to test if the bimodal motor-goal representations could be an averaging effect across trials in which the monkeys randomly switched between planning a direct or inferred reach. To test the sensitivity of the DMC similarity analysis, we applied it to neuronal data of the DMG task. First, the mean firing rates for each reach direction in DMG without distinction between direct-cued and inferred-cued trials were used to calculate mean DMC values. The idea of this was to simulate a scenario in which a bimodal selectivity profile is artificially created as an artifact of averaging across direct- and inferredchoice trials. Second, all correct DMG trials were sorted according to the choice of the monkey (as instructed by the context cue prior to the memory period in DMG trials) to compute choice-selective DMC values. Since neurons in PRR and PMd typically showed motor-goal selectivity in the DMG task, choice-selective profiles were unimodal with high absolute values of the DMC (Fig. S2). As expected, the differences between the choice-selective and choice-indifferent DMC values (see Methods) are significantly different from zero for PRR ( $p_{\text {direct }}=0.0012$; $\left.p_{\text {inferred }}=0.0007\right)$ and PMd $\left(p_{\text {direct }}=0.0032 ; p_{\text {inferred }}=0.0018\right)$. This shows that our DMC correlation method is a sensitive measure, which would detect trial-to-trial guess-andswitch strategies if present. 
A bimodal selectivity profile of a neuron could also be the consequence of a rapid behavioral switching between the two possible motor goals during single trials. To rule this out, we computed the variance of the spike densities in trials with reaches to the direction of maximum response (MD) and to the opposite direction (OD) in the PMG task, and compared it with the variance in the MD and OD trials in the DMG task. The variance was computed across time (within the memory period) and across trials, without differentiating between direct-cued and inferred-cued trials. The variances in the DMG task was expected to be high, since MD and OD by definition induce different spike rate levels. If the switching hypothesis was true, then the variance in the PMG trials should be similarly high. If genuine potential motor goal encoding was true, then the variance in PMG trials should be smaller than in the DMG task, since MD and OD trials in PMG would induce similar spike rate levels. We tested our prediction by analyzing the normalized variance

$$
\operatorname{Var}_{\text {norm }}=\frac{\log (\operatorname{var}(\text { spikedensity }))}{\log (\text { mean }(\text { spikedensity }))}
$$

during the last $600 \mathrm{~ms}$ of the memory period. With the normalization we compensated for the fact that the variance in our data increased with increasing mean spike rate, like it has been shown for V1 and MT neurons (Snowden and Hess, 1992). We extended the analysis period to $600 \mathrm{~ms}$, compared to the $300 \mathrm{~ms}$ for all other analyses, to take into account possible longer switch periods and to improve the statistical power.

For the balanced dataset in PRR we found that the variance in PMG was on average smaller than in DMG $\left(\mathrm{p}=1.84 \times 10^{-7}\right.$, paired Wilcoxon signed rank test; Fig. $\mathrm{S} 2 \mathrm{~b})$. This difference in the spike rate variance argues against the hypothesis that the bimodal selectivity profiles in PRR are mostly a consequence of averaging across short-term alternating choice-selective responses within or across trials. Instead it 
suggests that in PRR both potential motor goals are represented simultaneously within in each trial in the balanced dataset.

In PMd the variance in PMG trials was not lower than in DMG trials $(p>0.05$; Fig. S2d). This could be caused by slightly stronger trial-to-trial choice-selective signals, which the model-based analysis suggested to be present in PMd (see also Supplemental Information S1; Fig. S1b right). The stronger the choice-selective signal the higher the variance. This makes the variance measures in PMG and DMG more similar and reduces the distance to the unity line. 
a

$D M G_{\text {mean }}$ correlated with $D M G_{\text {direct }}$ and $D M G_{\text {inferred }}$ (PRR; $n=97$ )

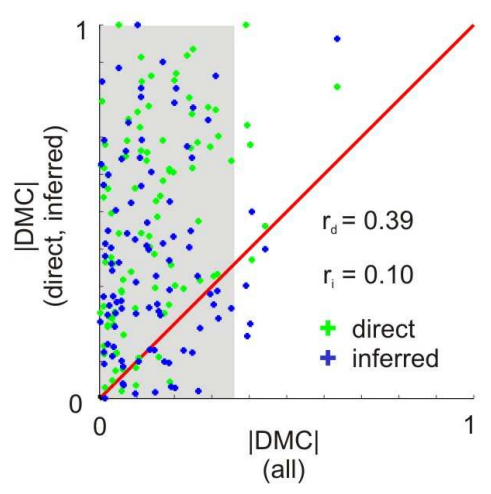

C

$D M G_{\text {mean }}$ correlated with $D M G_{\text {direct }}$ and $D M G_{\text {inferred }}$ (PMd; n=45)

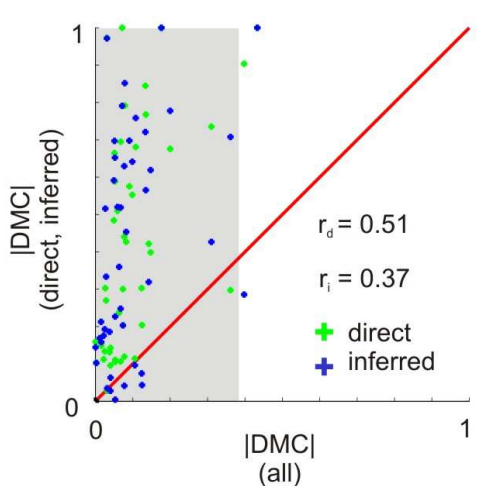

b

PMG vs. DMG pairwise (PRR)

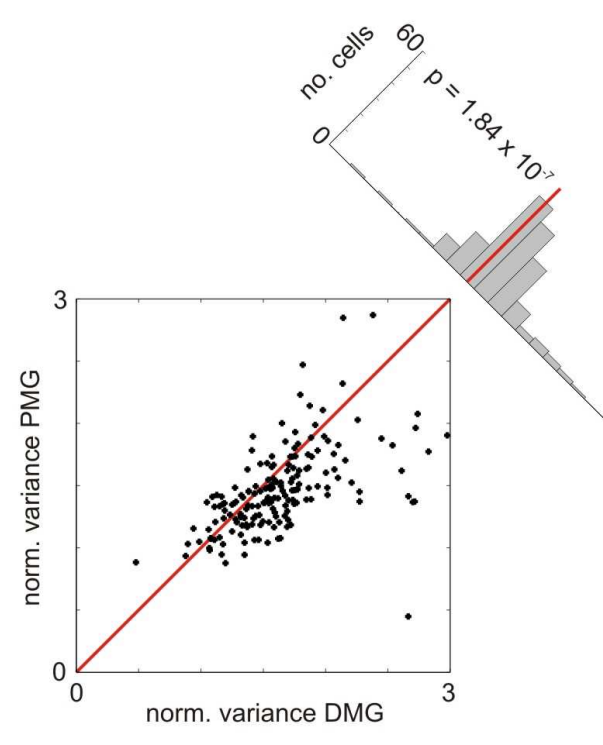

d

PMG vs. DMG pairwise (PMd)
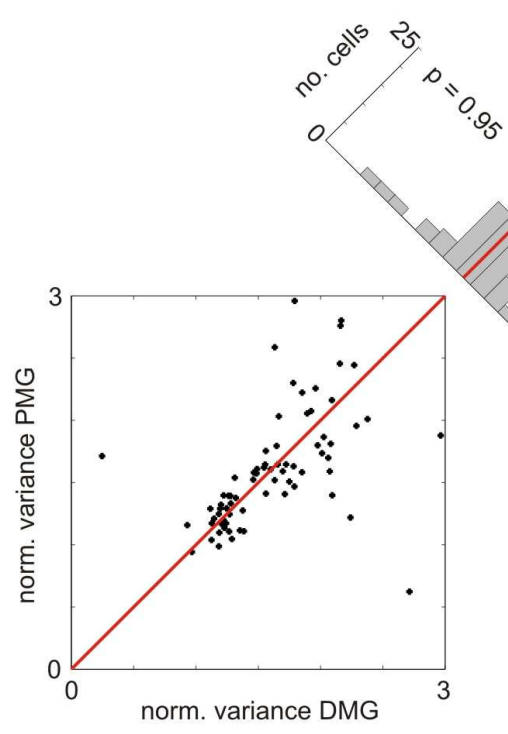

Figure S2: Control measures to rule out the selection encoding hypothesis. (a) DMC similarity analysis for the balanced dataset in the DMG task. The scatter of the data along the vertical axis demonstrates the sensitivity of this measure (see text for explanation). Same conventions as in Fig. 4b and 6c apply. (b) Within-trial motorgoal switching analysis. The normalized variances for each neuron in MD and OD trials are compared between the DMG and PMG task (see text). Top right shows the 
distribution of the variance differences between DMG and PMG for all neurons. The p-value denotes the result of a paired Wilcoxon sign rank test. 


\section{Supplemental Information S3 - Source of the behavioral bias in the biased dataset (related to Fig. 5a)}

Even though direct or inferred context instructions were equally likely in PMG-CI trials, and both choices were rewarded with equal amount and probability in PMG-NC trials, the monkeys' choice behavior and neuronal selectivity profiles showed a strong preference for the inferred reach goal in the biased dataset. At first glance, the preference for inferred reaches seems counter intuitive. In natural environments direct reaches are more common, and are easier to perform, since they do not require a spatial transformation.

One advantage of planning the less intuitive inferred reaches by default might be the preparation of the more difficult response alternative in order to comply with any constraint on reaction times after the 'GO'-signal. Alternatively, the more intensive training of the inferred task could have initially biased the monkeys. In the Bayesian sense, the frequency of exposure to inferred trials during training might have acted as prior probability which biased the decision (Körding and Wolpert, 2004; Diedrichsen et al., 2010). Since in the EPRS any choice strategy yields the same average reward, the monkeys did not have an incentive to deviate from any pre-existing behavioral bias. Even worse, any pre-existing bias should become enhanced since the monkeys, due to their bias, make the experience that more trials of the type for which they have a bias are being rewarded (simply because the monkey makes more of these trials).

Note, that our conclusions in the main manuscript are independent of the origin of the behavioral bias. Nevertheless, we believe that a combination of two of the above effects can be seen in our experiment. When, after several month of showing balanced 
behavior in the BMRS, one or our monkeys returned to the EPRS, he quickly developed a strong inferred-choice bias again (Fig. S3). This can hardly be explained with an imbalance between direct-cued and inferred-cued trials in the initial training stages. Rather, we interpret this as indication for a strategic advantage of planning inferred reaches in PMG trials. An initial small bias induced by this asymmetry might be self-enhanced via the Bayesian prior probability effect.

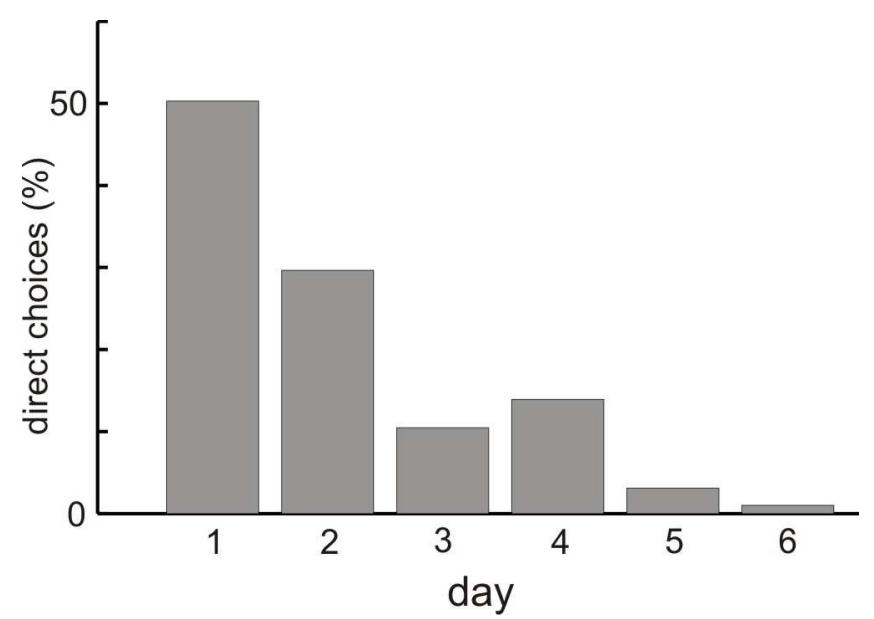

Figure S3: Re-occurrence of the inferred-choice bias in behavior when returning to the EPRS after several months of BMRS. The bars show the percentage of direct choices over six consecutive training days with an EPRS. Starting from a balanced behavior the monkey quickly developed a strong inferred-choice bias, even though direct-choices and inferred-choices were rewarded equally. 


\section{Supplemental Information S4 - Normalization and scaling factors (related to figures $3 b$ and $5 b$ )}

The example neurons in Figures $3 b$ and $5 b$, and the activity plots in Figures $3 c$ and $5 c$ show that the activity during the memory period is lower for two equally preferred motor goals than for a single preferred goal. To quantify this, we analyzed the distribution of the scaling factors $A$ that were used for the model based analysis (see SI 1). The scaling factor was the fitted parameter in this analysis, and it quantifies the relative response strength of each neuron between the DMG and PMG tasks. For the inferred model (and also the direct model, which was never the dominant model, and therefore is not further considered) a scaling factor of 1 (one) means that the activity in the PMG task is not different from the DMG task. Instead, a scaling factor of 1 (one) in the mean model indicates halving of the activity in the DMG compared to the PMG task (see Experimental Procedures).

Figure S4 shows the distribution of scaling factors for the inferred model (S4a; biased dataset) and the mean model (S4b; balanced dataset). The mean scaling factors did not significantly deviate from 1 in either case, which indicates that the neural response strength on average is only half as strong when two equally preferred motorgoals are present, compared to a single one. This argues in favor of a mutual competition between multiple motor goals, as implemented in dynamic field models (Erlhagen and Schoner, 2002; Cisek, 2006) or other models of decision making (Averbeck and Seo, 2008; Eliades and Wang, 2008). This competition seems to be active even during reach planning, well before the final decision is enforced after the 'GO'-signal. 
a

model scaling factors biased dataset (inferred model)

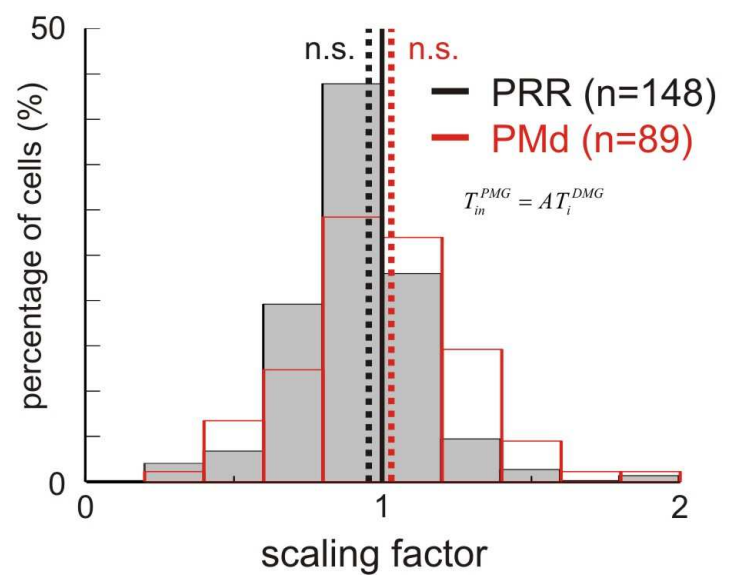

b model scaling factors
balanced dataset (mean model)

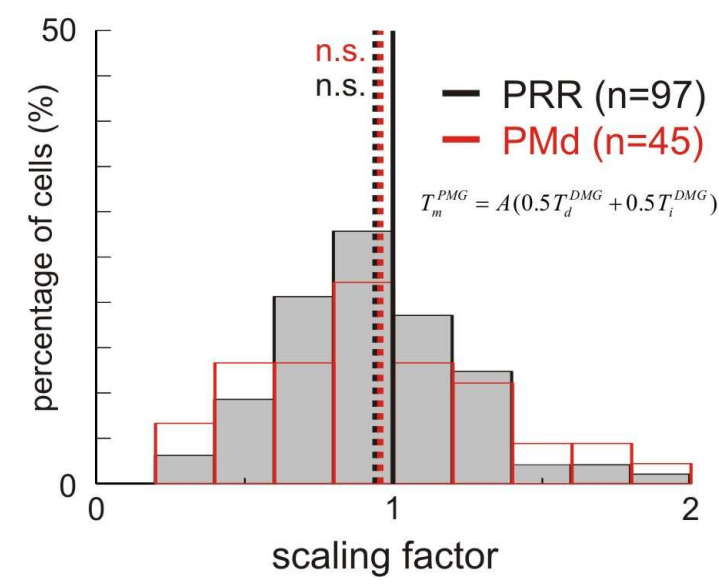

Figure S4: Scaling factors for model fit. (a) Distribution of the best-fit scaling factors (A in the linear model) of the inferred model for the biased dataset in PRR (grey; black outline) and PMd (blank; red outline). (b) Distribution of scaling factors A of the mean model for the balanced dataset (see Methods). The dotted lines indicate the mean values of the distributions, none of which significantly deviated from 1 . This means that the neural response strengths to either of the two potential motor goals in the balanced data set were on average half as strong as the responses to a unique single motor goal in the biased dataset. 


\section{Supplemental Information S5 - Simultaneous behavioral and neural data collection with biased dataset (related to Figure 5)}

In the main manuscript, behavioral testing of choice preferences with PMG-NC trials in the dataset with equal probability reward strategy (EPRS) was introduced at the end of the corresponding neural collection period. To ensure that independent behavioral and neural data collection causes no confounds on our interpretation, we also collected one biased dataset with the EPRS and strictly parallel neuronal and behavioral recordings (monkey A). This EPRS had a $100 \%$ reward probability for direct and inferred reaches, and showed a strong behavioral bias for inferred choices (Fig. S2a), i.e. most reaches were directed towards the inferred motor goal $(80 \pm 4.6$ $\%)$ and only a small fraction towards the direct goal $(1.6 \pm 1 \%)$, as in the EPRS-50 dataset of the main manuscript. The equivalent choice bias between the EPRS-50 and EPRS-100 datasets is not surprising. Neither EPRS pushes a monkey to change its choice behavior, since the reward is independent of the pursued behavior. The distribution of direction modality contrast (DMC) values in the control experiment $\left(\mathrm{m}=-0.27 ; \mathrm{p}=8.9 \times 10^{-4} ;\right.$ Fig. $\left.\mathrm{S} 2 \mathrm{~b}\right)$ was qualitatively the same as in the biased dataset of the main manuscript (Fig. 4d). The model based analysis also yielded equivalent results between the main and control data (cp. Figs. S5c and S1a). This control experiment shows that simultaneous behavioral testing and neural recording in PMGNC and PMD-CI trials yields identical results to subsequent testing and recording, and that the overall reward probability in an EPRS does not affect the results. 
a

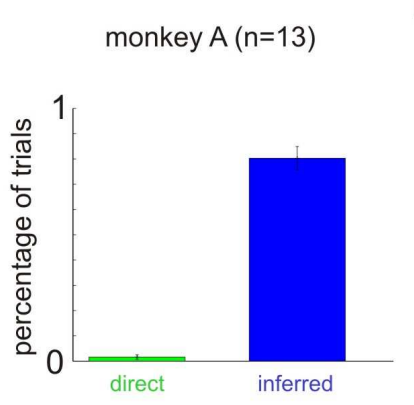

b

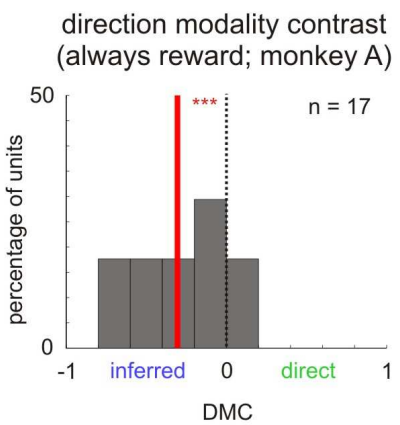

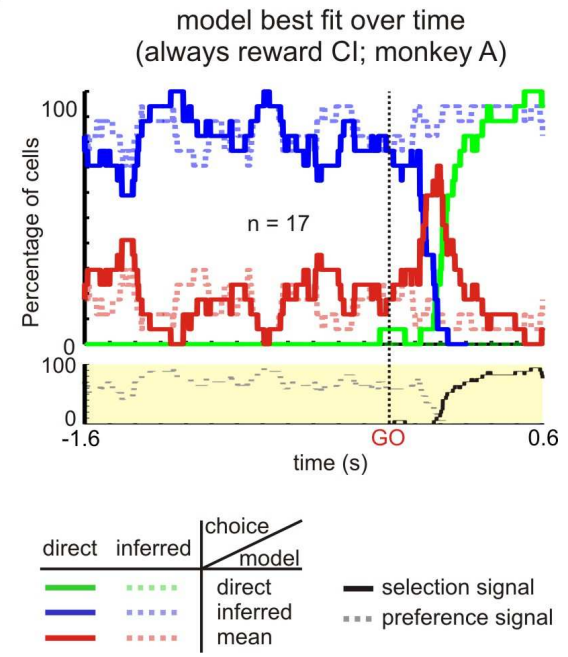

Figure S5: Preference encoding for a dataset with simultaneous recording of behavioral and neural recording during an EPRS in PRR of monkey A. Same conventions as in Figs. 5 and S1 apply. (a) The behavioral bias was the same when $100 \%$ reward for either the direct or inferred motor goal was applied compared to when the $50 \%$ EPRS was used (5a). The DMC values (b) and the model-based analysis (c) derived from neurons recorded in parallel with behavioral testing yielded the same results as for the subsequent testing (Fig. 5c). 


\section{Supplemental Information S6 - Alternative definitions of selection and options encoding (related to Methods)}

For our analyses we had two distinct criteria that neurons had to fulfill to be included. First neurons had to be directional selective in the DMG task. This criterion was necessary because we only wanted to analyze neurons which were related to the reach task. Also, we needed a defined preferred direction to calculate the DMC values and selectivity profiles for the model based analyses, which we could only determine from neurons which were directionally selective in the DMG task. Second, neurons also had to be directionally selective in the PMG task. This restriction ensured that nonselective neurons were separated from bimodal neurons, which would both have a DMC value close to zero.

We also used two alternative neuron selection criteria. We did this in order not to exclude neurons which could have counted for the selection or option hypotheses, if these hypotheses were defined less strictly. First, we analyzed all neurons which were directionally selective in the memory period of DMG trials and after the 'GO'-signal in PMG trials, but not in the PMG memory period. These cells were only active when a definite motor goal was defined, but not when a preliminary (potential) motor goal existed, which could be interpreted as definite selection encoding (as opposed to a preliminary selection signal during the memory period of the PMG task). These criteria applied to only a small fraction of neurons in PRR (9\% biased; $4 \%$ balanced) and PMd (3\% biased; $10 \%$ balanced), which does not change our interpretations. Second, we analyzed all neurons which were not directionally selective in the DMG task and also not in the PMG task after the 'GO'-signal, but in the PMG task during the memory period. These cells were only active when preliminary options existed, 
but not when a definite motor goal was defined, which could be interpreted as pure options encoding, which would only come into effect when more than one motor goal option is available. These criteria, too, applied only to small fractions of neurons in PRR (5 \% biased; $2 \%$ balanced) and PMd (10\% biased; $2 \%$ balanced), which also does not change our interpretations of the main manuscript. 
Reference List

Averbeck,B.B. and Seo,M. (2008). The Statistical Neuroanatomy of Frontal Networks in the Macaque. PLoS Computational Biology 4, e1000050.

Cisek,P. (2006). Integrated Neural Processes for Defining Potential Actions and Deciding between Them: A Computational Model. Journal of Neuroscience 26, 97619770.

Diedrichsen,J., White,O., Newman,D., and Lally,N. (2010). Use-Dependent and Error-Based Learning of Motor Behaviors. Journal of Neuroscience 30, 5159-5166.

Eliades,S.J. and Wang,X. (2008). Chronic multi-electrode neural recording in freeroaming monkeys. J Neurosci Methods 172, 201-214.

Erlhagen,W. and Schoner,G. (2002). Dynamic field theory of movement preparation. Psychological Review 109, 545-572.

Körding,K.P. and Wolpert,D.M. (2004). Bayesian integration in sensorimotor learning. Nature 427, 244-247.

Snowden,R.J. and Hess,R.F. (1992). Temporal frequency filters in the human peripheral visual field. Vision Research 32, 61-72.

Szucs,A. (1998). Applications of the spike density function in analysis of neuronal firing patterns. J Neurosci Methods 81, 159-167. 


\section{Reference frames}

\section{II.I. Reach planning in different reference frames}

To perform a reach movement one has to move the hand from the current position to the desired target position. To perform this movement the brain has, first, to encode both the position of the hand and of the target, and second, the difference vector between both positions. This motor error, i.e. the vector that points from the hand to the target position, can be described in intrinsic coordinates. In this sense it would be encoded in the specific pattern of muscle activation or changes of joint angles, which would be required to reach to the target. However, if we plan a reach we normally do not think about muscle activation or changing of joint angles, but we think in extrinsic coordinates about moving the hand from one position to the other. There is evidence that movement plans in the parietal and premotor cortex are also encoded in extrinsic space. A position in extrinsic space can be encoded with respect to different reference points. For example hand and target could be represented in relation to the gaze direction (eye-centered reference frame) or in relation to some body part as the shoulder (body-centered reference frame). In both reference frames the target position is encoded independent of the hand position. To achieve the motor error, an inseparable combination of hand and target representation is necessary. Once this is achieved the motor error itself is coded with respect to the hand position (handcentered reference frame).

Tuning properties of neurons in the posterior parietal cortex seem to be ideal for mediating the transformation from a separate representation of hand and target position (in eye-centered reference frame) to a representation of the motor error (in hand-centered reference frame). In PRR most cells encode the target position in an eye-centered reference frame (Batista et al., 1999; but see also Chang and Snyder, 2010). The activity of those cells is gain modulated by the initial hand position. Thus, the tuning of a neuron does not change with initial hand position (i.e. it will always respond best at a certain target position relative to the gaze) but the overall activity of the neuron does (i.e. the neuron is less active if the hand is for example on the right side compared to the left side). This leads to separate coding of eye and hand positions. In the adjacent area 5, the activity of the cells is best described by a combination of the hand- and eye-position and - in its extreme case - the coding of 
the target position in a hand-centered reference frame. The intermediate neurons likely play a critical role in the transformation from an eye- to a hand-centered representation. Their tuning properties are consistent with those of neurons in intermediate layers of artificial neural networks which perform such a transformation (Zipser and Andersen, 1988; Burnod et al., 1999). Neurons in PMd have been shown to mostly encode a combination of eye- hand- and target- position, but there are also neurons which are eye-centered and others, which are hand-centered.

Behavioral parameters in a reaching task reflect more or less strongly the one or other part of the movement process. Reaction times e.g. are closer to the planning stage since they are measured before movement execution. Movement times or reach precision, in contrast, although influenced by the movement planning, are also strongly affected by movement execution. How reach goal encoding in different reference frames in different brain areas transfer to behavioral parameters is not obvious. Several studies investigated influences of reference frames on reach endpoints and systematic reach errors. Depending on their tasks and analyses, the reference frames which influenced the reach parameters were different. Some studies suggest that the remembered target location for reaches is coded in an eye-centered reference frame (Henriques et al., 1998; Beurze et al., 2006; Sorrento and Henriques, 2008) Other experiments demonstrate influence of a hand-centered reference frame (Bock and Eckmiller, 1986) or indicate that reach planning can be achieved using a combination of multiple reference frames (McGuire and Sabes, 2009). These different results may be explained by the different manipulations in each task, which affect different stages of the processing pathway. Previous studies mostly compared two or more reference frame by analyzing one behavioural parameter. To see how different reference frames affect different behavioural parameters, we did a psychophysical study in which the influence of an eye-centered and hand-centered reference frame on reaction times, movement times and reach precision was compared. 


\title{
What is 'anti' about anti-reaches? Reference frames selectively affect reaction times and endpoint variability
}

\author{
Stephanie Westendorff · Alexander Gail
}

Received: 15 June 2010 / Accepted: 25 October 2010 / Published online: 13 November 2010

(C) The Author(s) 2010. This article is published with open access at Springerlink.com

\begin{abstract}
Reach movement planning involves the representation of spatial target information in different reference frames. Neurons at parietal and premotor stages of the cortical sensorimotor system represent target information in eyeor hand-centered reference frames, respectively. How the different neuronal representations affect behavioral parameters of motor planning and control, i.e. which stage of neural representation is relevant for which aspect of behavior, is not obvious from the physiology. Here, we test with a behavioral experiment if different kinematic movement parameters are affected to a different degree by either an eye- or hand-reference frame. We used a generalized anti-reach task to test the influence of stimulus-response compatibility (SRC) in eyeand hand-reference frames on reach reaction times, movement times, and endpoint variability. While in a standard anti-reach task, the SRC is identical in the eye- and hand-reference frames, we could separate SRC for the two reference frames. We found that reaction times were influenced by the SRC in eye- and hand-reference frame. In contrast, movement times were only influenced by the SRC in hand-reference frame, and endpoint variability was only influenced by the SRC in eye-reference frame. Since movement time and endpoint variability are the result of planning and control processes, while reaction times are consequences of only the planning process, we suggest that SRC effects on reaction times are highly suited to investigate reference frames of movement planning, and that eye- and hand-reference frames have distinct effects on different phases of motor action and different kinematic movement parameters.
\end{abstract}

\footnotetext{
S. Westendorff · A. Gail ( $\square)$

Bernstein Center for Computational Neuroscience Göttingen, German Primate Center, Kellnerweg 4,

37077 Göttingen, Germany

e-mail: agail@gwdg.de
}

Keywords Reach planning $\cdot$ Stimulus-response compatibility $\cdot$ Reference frames $\cdot$ Sensorimotor transformation $\cdot$ Eye-hand coordination

\section{Introduction}

To successfully plan and control goal-directed reach movements, one has to estimate the motor error between the current hand position and the target position. One assumption is that in order to compute the difference vector, the hand and target positions have to be represented in a common reference frame (Buneo and Andersen 2006). This might be a representation in eye-, shoulder- or some other body-centered reference frame. A lesion study (Khan et al. 2005) and imaging data (Medendorp et al. 2003) from humans and electrophysiological studies in monkeys (Batista et al. 1999; Buneo et al. 2002; Pesaran et al. 2006) showed that the parietal reach region (PRR) of the posterior parietal cortex encodes planned reach target locations predominantly relative to the direction of the gaze. This suggests that PRR represents a stage of reach planning prior to the definition of the motor error in a hand-reference frame. In parietal area 5 (Buneo et al. 2002) and the dorsal premotor cortex (Batista et al. 2007; Pesaran et al. 2006), the reach target location in a hand-reference frame contributes stronger to the spatial representations, often resulting in a combined encoding of eye, hand and target position.

Which level of processing finally is responsible for which aspect of behavioral performance is not clear from these neurophysiological observations. There is psychophysical support for the encoding of remembered reach target locations in an eye-reference frame (Beurze et al. 2006; Henriques et al. 1998; Sorrento and Henriques 2008), while other experiments showed an influence of 
hand-reference frame (Bock and Eckmiller 1986), or indicate that reach planning can be achieved in a combination of multiple reference frames (McGuire and Sabes 2009). The latter result suggests that the contribution of each reference frame depends on the available information in that reference frame. If the reach target was defined visually, the eye-centered representation was weighted stronger than a body-centered representation, and vice versa, if the reach target was defined by a proprioceptive target, the bodycentered representation gained more weight. These previous psychophysical studies used systematic reach endpoint errors for determining the reference frame of movement planning. Endpoint errors not only depend on movement planning, but also motor control. Similarly, movement times (MTs) and endpoint variability (EVs) reflect both planning and control processes of the movement. Reaction times (RTs), in contrast, cannot be influenced by motor control processes, since they are measured before onset of the movement and thereby allow isolating the influence of reference frames during the planning process.

We tested the hypothesis that different movement parameters are differently influenced by an eye- and handcentered reference frames. Alternatively, a single reference frames could affect multiple parameters of movement planning and control in the same way, e.g. resulting from a task-specific cognitive strategy or selective availability of different sensory input signals (McGuire and Sabes 2009). Going beyond previous studies, we designed an experiment in which we could compare the influence of eye- and handcentered reference frames on RTs, MTs and EVs within the same behavioral task.

We designed a new pro-/anti-reach task with which we could modify spatial stimulus-response compatibility (SRC) separately in an eye- and hand-reference frame (Lamberts et al. 1992; Nicoletti and Umilta 1989; Umilta and Liotti 1987). The idea is that SRC in a certain reference frame (e.g. eye-reference frame) only should have an effect on a certain parameter (e.g. RT) if this reference frame is functionally relevant for the respective movement parameter. Furthermore, the idea is that the reference frame which contributes to SRC effects in a certain movement parameter is also the reference frame of the neuronal representations underlying this movement parameter. In this sense, an influence of a certain reference frame on a behavioral parameter could help to relate behavioral parameters to brain areas with activity pattern in the same reference frame.

Spatial compatibility between the instruction stimulus (cue) and the associated behavioral response is known to influence RTs in various types of tasks. Subjects are in general faster if the spatial information contained in a visual cue matches spatial response parameters, independent of the exact type of movement to be performed (Duncan 1977; Fitts and Deininger 1954; Fitts and Seeger 1953; Georgop- oulos et al. 1989; Hommel 1996; Lamberts et al. 1992; Morin and Grant 1955; Nicoletti and Umilta 1984; Proctor and Vu 2002; Shaffer 1965). In pro-/anti-paradigms (Crammond and Kalaska 1994; Everling et al. 1998; Fischer and Weber 1992; Gail and Andersen 2006; Hallett 1978; Zhang and Barash 2000), a pro-response is directed toward a spatial stimulus, whereas an anti-response is directed opposite to the spatial stimulus. In contrast to button-presses or joystick experiments, subjects in pro-/anti-reach tasks execute reach movements in the same workspace as the visual instructions are given, which makes eye- and hand-visuospatial reference frames more comparable.

In a standard pro-/anti-reach task, the SRC is identical in the eye- and hand-reference frame. We developed a generalized pro-/anti-reach task to dissociate the influence of eye- and hand-reference frame on SRC effects. With the task design, we could define reaches that were compatible in one reference frame, but not the other, and vice versa. Viewed from a slightly different perspective, the generalized pro-/anti-reach task allows answering the question of what makes an anti-reach incompatible, the incompatibility of cue and response in the eye- or hand-reference frame. We found that SRC in eye- and hand-reference frames affected RTs, MTs and EVs in a distinct manner, indicating that different aspects of movement planning and control are influenced by the two reference frames to a different degree.

\section{Methods}

\section{Subjects}

Sixteen right-handed subjects (7 females, 22-38 years) with normal or corrected-to-normal vision participated in the main experiment, 15 ( 8 females, $21-27$ years) in a control experiment. All were naïve with respect to the objective of the study. Detailed written instructions were given to the subjects before the experiment. Subjects had the opportunity to get familiar with the setup and practice the task for about $15 \mathrm{~min}$. All subjects had a success rate higher than $70 \%$ during training, which was a prerequisite for participation in the recording session. Experiments were in accordance with institutional guidelines for experiments with humans and adhered to the principles of the Declaration of Helsinki. All subjects gave their informed consent prior to their inclusion in the study.

Generalized pro- and anti-reach task

In a choice reaction-time task subjects had to perform reaches with their preferred hand on a touch screen. Reaches were instructed by two visual cues: A colored context cue (green or blue square frame around eye-fixation 
point, $\sim 3^{\circ}$ visual angle (VA) edge length) indicated whether the subjects had to make a reach into the same (pro-reach, green) or opposite (anti-reach, blue) direction of a spatial cue. The spatial cue (white circular patch, diameter of $\sim 3^{\circ} \mathrm{VA}$ ) instructed the movement direction in an eyecentered reference frame.

Eye- and hand-fixation stimuli were presented at $\pm 5 \mathrm{~cm}$ $\left(7^{\circ} \mathrm{VA}\right)$ relative to the screen center, spatial cues at $0 \mathrm{~cm}$ (screen center) or $\pm 10 \mathrm{~cm}$. Over the whole experiment, the three potential spatial cue positions were also potential reach target positions. But in each individual trial only the two cue positions at $\pm 5 \mathrm{~cm}$ to the eye stimulus served as potential cue positions. Therefore, the spatial cue appeared always at the same visual eccentricity. Similarly, in each trial only the two target positions at $5 \mathrm{~cm}$ to the left or right of the hand-fixation position served as potential reach goals, such that all reaches had the same reach amplitude of $5 \mathrm{~cm}$, and a $50 \%$ probability of leftward or rightward direction.

Half of the trials were standard pro-/anti-trials. In standard pro-/anti-trials, the eye- and hand-fixation points were identical, either at the $+5 \mathrm{~cm}$ (right fixation) or at the $-5 \mathrm{~cm}$ (left fixation) screen position. A spatial cue appeared either left or right of the fixation points, and subjects had to make a movement in the same (pro) or opposite (anti) direction. A standard pro-reach is compatible in eye- and hand-reference frame, since cue and target are identical. A standard anti-reach is incompatible in eye- and hand-reference frame, since cue and target lie in opposite directions with respect to gaze and hand starting position.

The other half of the trials were generalized pro-/antitrials in which the compatibility in an eye-reference frame can be dissociated from compatibility in a hand-reference frame. In the generalized pro-/anti-reach, eye- and handfixation points were separated. Subjects had to eye-fixate at the $-5 \mathrm{~cm}$ and hand-fixate at the $+5 \mathrm{~cm}$ screen position, or vice versa. The instruction for the generalized pro-/antireach was the same as for the standard pro-/anti-reach: Subjects had to reach in the same or opposite direction of the cue. Note, for the purpose of instructing the subjects, the spatial cue direction was defined relative to the eyefixation position (solid black arrow; Fig. 1a), and the reach direction was defined relative the to hand-fixation position (open gray arrow). For the purpose of analyzing the data, and different from the task instruction, a trial was defined as compatible/incompatible in eye-reference frame if the direction of the spatial cue and the reach goal both were the same/opposite in relation to the eye-fixation stimulus (black arrows, Fig. 1b). And a trial was defined as compatible/ incompatible in hand-reference frame if the direction of the a
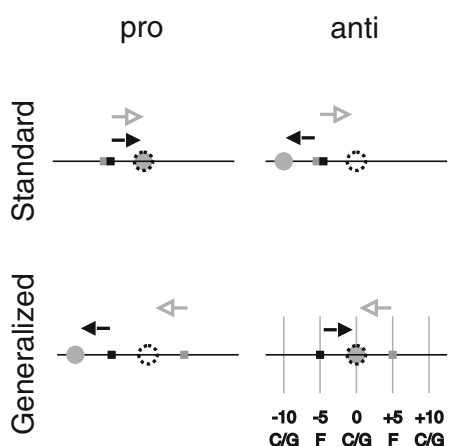

b

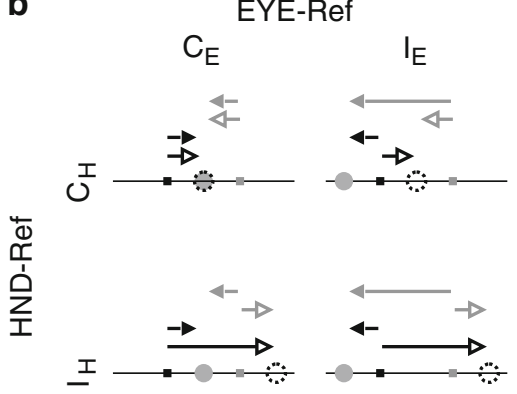

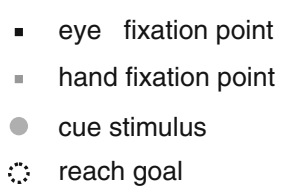

- eye fixation point

- hand fixation point

reach goa $\rightarrow$ direction of spatial cue in eye reference frame

$\rightarrow$ direction of reach goal in eye reference frame

$\rightarrow$ direction of spatial cue in hand reference frame

$\rightarrow$ direction of reach goal in hand reference frame
Fig. 1 Spatial layout of the generalized pro-/anti-reach task. a General task design in standard and generalized trials. Squares depict positions where eye- and/or hand-fixation points $(F)$ were presented. Gray circles depict the positions where the spatial cue $(C)$ was presented. Dotted circles depict the positions of the reach goal $(G)$. In the lower right panel, the $5 \mathrm{~cm}$ raster is illustrated at which the fixation points, cues, and goals could be positioned. For simplicity, each panel only shows one out of four possible spatial configurations (fixation left/ right, cue left/right) for each of the four task conditions (pro/ anti $\times$ standard/generalized). The task design consists of a total of 16 conditions. In a, black arrows indicate the direction of the spatial cue relative to eye fixation, and the gray arrows the direction of the reach goal relative to hand fixation. In pro-trials (per definition) both arrows point in the same direction, whereas in anti-trials, they point in opposite directions. b Four example trials in the generalized task condition, which illustrate the $2 \times 2$ variations of the SR compatibility in the eye-reference frame (black arrows) and in the hand-reference frame (gray arrows). The open arrows show the direction of the spatial cue, the solid arrows show the direction of the reach goal 
spatial cue and the reach goal were the same/opposite in relation to the hand-fixation stimulus (gray arrows). The separation of eye fixation from hand fixation in the generalized conditions, in combination with the pro-/anti-rule, leads to reaches, which were compatible in eye-reference frame, but incompatible in hand-reference frame $\left(\mathrm{C}_{\mathrm{E}} \mathrm{I}_{\mathrm{H}}\right.$; Fig. 1b), and reaches, which were compatible in hand-reference frame, but incompatible in eye-reference frame $\left(\mathrm{I}_{\mathrm{E}} \mathrm{C}_{\mathrm{H}}\right)$. There were also reaches, which were compatible in eyeand hand-reference frame $\left(\mathrm{C}_{\mathrm{E}} \mathrm{C}_{\mathrm{H}}\right)$ and reaches, which were incompatible in eye- and hand-reference frame $\left(\mathrm{I}_{\mathrm{E}} \mathrm{I}_{\mathrm{H}}\right)$, like in the standard pro-/anti-task. Note that the compatibility of cue and reach goal in either reference frame refers only to compatibility with respect to the direction of cue and reach goal. The distance of the cue from the eye-/hand-fixation point can be different than the distance of the reach goal from the respective fixation point (see arrow length in Fig. 1b).

The timeline of the reaction-time task is shown in Fig. 2. The subject initiated a trial by fixating a small, red fixation spot and touching a white hand target (fixation period). After a random delay $(0.5-1.0 \mathrm{~s})$, the context cue was briefly flashed (pre-cue period, $0.2 \mathrm{~s}$ ). The context cue was presented early to induce the effects of compatibility even in a paradigm in which compatible and incompatible conditions were interleaved randomly (de Jong 1995; Proctor and

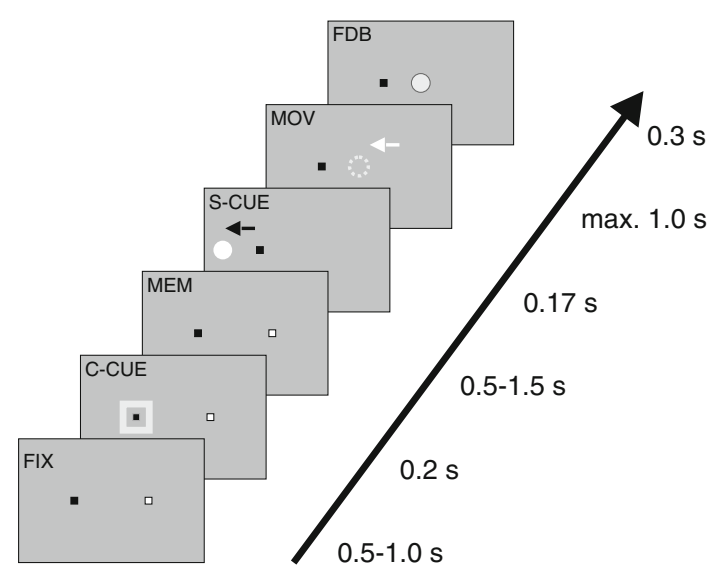

Fig. 2 Timeline of the generalized pro-/anti-reach task. The subject had to direct gaze to a small red square throughout the trial. A flashed context cue (C-CUE, frame around the eye-fixation) instructed whether to prepare a pro- or anti- reach. In the experiment, a green frame instructed a pro-reach and a blue frame instructed an anti-reach. The reach goal (dotted circle, not visible to the subject) was defined by the combination of the context cue and a spatial cue (S-CUE, white circle), which was flashed left or right of the eye fixation after a variable memory period $(M E M)$. Visual feedback $(F D B)$ appeared only after the subject touched the correct reach goal on the screen. Pro- and anti-reaches were defined as a reaches relative to hand fixation (symbolized by white arrow, not shown to subjects) in the same (pro) or opposite (anti) direction as the spatial cue was relative to eye fixation (symbolized by black arrow, not shown to subjects). The example shows a generalized pro-trial
Vu 2002; Shaffer 1965). For a variable duration, the subject had to keep the hand fixation (memory period, $0.5-1.5 \mathrm{~s}$ ) until the hand target turned off (go-signal) and simultaneously the spatial cue was flashed (go-cue period, $0.17 \mathrm{~s}$ ). After the go-signal, the subject had to reach toward the cued reach goal location (movement period, max. $1.0 \mathrm{~s}$, including reaction and movement time). The hand had to be kept at the reach goal location (feedback period, $0.3 \mathrm{~s}$ ) to successfully finish the trial. The subject received visual feedback about the correct reach goal, consisting of a circular patch stimulus at the reach goal location presented immediately after acquiring the desired position. If the subject did not reach the goal location before the maximum movement period expired, then the trial was aborted immediately. An auditory feedback (high/low pitch tone) indicated whether the trial was correct or not.

All parameters of the task (standard/generalized, pro/anti, cue left/cue right, fixation left/ fixation right) were randomly interleaved. Only correct trials were analysed, and each subject performed about 20 correct trials per condition.

\section{Visual display and behavioral control}

Visual instruction stimuli were presented on a LCD screen (19" ViewSonic VX922) mounted behind a touch screen (IntelliTouch, ELO Systems, CA, USA). Custom-written display software $(\mathrm{C}++)$ was controlled via a real-time LabView control program running on a PXI computer (National Instruments). The display of the stimuli was synchronized with the vertical synchronization of the screen to avoid latency jitter. Visual display latencies were recorded with a photodiode and corrected for in the data analysis. All visual instruction stimuli had high contrast and were readily visible. Subjects were seated in front of a fronto-parallel touch screen $(40 \mathrm{~cm}$ distance from eye, screen center at eye level) with a chinrest to minimize head movements. Reaches were not constrained in any specific manner other than the touch positions on the touch screen.

Hand position was registered with the touch screen and monitored within the real-time control software. The hand fixation and reach targets had to be continuously touched within a tolerance window of typically $3 \mathrm{~cm}\left(4.0^{\circ} \mathrm{VA}\right)$ radius. Otherwise the trial was immediately aborted. Reaction time (RT) was defined as the time between the go-signal and the subject's release of the touch screen from the fixation position. Movement time (MT) was defined as the time between the release and re-acquisition of the touch screen at a target position. Endpoint variability (EV) was defined as variable reach error, i.e. the distance of reach endpoint in each trial to the mean reach endpoint to the same reach target. EVs within each subject were calculated separately for the $x$ - and $y$-dimension. RTs, MTs and EVs were calculated separately for each task condition. 
To control for possible effects of eye movements in the main dataset, a second group of 15 subjects was recorded in the same task but with eye movements being constrained. With this control experiment, we wanted to exclude the possibility that compatibility effects could be explained by reflexive saccades of the subjects toward the spatial cue location before executing the reach toward the reach goal. Such behavior would cause a delay in reach responses, since eye movements often lead and predict hand movements (Ariff et al. 2002), and reaction times in incompatible trials would be artificially prolonged, if the subjects executed two saccades (one reflexive to the spatial cue position and one corrective to the reach goal) before the start of the reach. In the control experiment, gaze direction was constrained to a tolerance window of $2 \mathrm{~cm}\left(\sim 2.8^{\circ} \mathrm{VA}\right)$ radius, otherwise the trial was immediately aborted $(500 \mathrm{~Hz}$ IR camera, SMI, Teltow, Germany).

\section{Data analysis}

In the standard pro-/anti-task, compatibility and incompatibility in eye- and hand-reference frames covary. We tested for the compatibility effects in eye- and hand-reference frames by comparing RTs, MTs and EVs in all compatible against all incompatible trials, independent of the direction of cue and reach (left/right) and laterality of fixation (left/ right), using a $t$ test. The main research question of our experiment regards the separation of compatibility effects in an eye-centered from compatibility effects in a hand-centered reference frame. For this, we tested RTs, MTs, and EVs with a repeated-measurement two-way ANOVA with the factors eye- and hand-reference frame in the generalized pro-/anti-task. Figure $1 \mathrm{~b}$ illustrates the $2 \times 2$ design of the ANOVA. The columns depict the conditions, which are compatible (left column) and incompatible (right column) in an eye-centered reference frame, whereas the rows depict conditions, which are compatible (upper row) and incompatible (lower row) in a hand-centered reference frame. Direction of cue and reach (left/right) and laterality of eye and hand fixation (left/right) were not treated as factors. Note, since in the standard task the compatibility in eyeand hand-reference frame always covary, we cannot simply expand the ANOVA to a third factor "standard/generalized", but instead have to analyse both data sets separately.

\section{Results}

The average success rate of the subjects in standard trials was $88 \pm 2 \%$ in pro and $88 \pm 1 \%$ in anti-reaches (mean $\pm \mathrm{SEM})$. First, we tested standard pro- and antireaches for SRC effects across all sample subjects (Fig. 3a, dashed line). RTs in standard pro-trials were on average faster than in standard anti-trials (pro: $326 \pm 11 \mathrm{~ms}$, anti: $355 \pm 11 \mathrm{~ms}$, mean $\pm \mathrm{SEM}, N=16, P=0.00016$, paired $t$ test). In the standard conditions, compatibility in eye- and hand-reference frame was always the same. To test compatibility effects in eye- or hand-reference frame, we analysed the generalized pro-/anti-conditions. In generalized trials, cue and reach goal were compatible in either eye- or handreference frame, but not in the other $\left(\mathrm{C}_{\mathrm{E}} \mathrm{I}_{\mathrm{H}}\right.$ or $\left.\mathrm{I}_{\mathrm{E}} \mathrm{C}_{\mathrm{H}}\right)$, or they were compatible in both reference frames $\left(\mathrm{C}_{\mathrm{E}} \mathrm{C}_{\mathrm{H}}\right)$, or they were incompatible in both reference frames $\left(\mathrm{I}_{\mathrm{E}} \mathrm{I}_{\mathrm{H}}\right)$. The average success rate was similar in those conditions $\left(\mathrm{C}_{\mathrm{E}} \mathrm{C}_{\mathrm{H}}\right.$ : $\left.87 \pm 2 \% ; \mathrm{C}_{\mathrm{E}} \mathrm{I}_{\mathrm{H}}: 84 \pm 2 \% ; \mathrm{I}_{\mathrm{E}} \mathrm{C}_{\mathrm{H}}: 89 \pm 2 \% ; \mathrm{I}_{\mathrm{E}} \mathrm{I}_{\mathrm{H}}: 87 \pm 2 \%\right)$. Figure 3 shows the RT results across all sample subjects. RTs were fastest if spatial cue and reach goal were compatible in both reference frames $\left(\mathrm{C}_{\mathrm{E}} \mathrm{C}_{\mathrm{H}}: 367 \pm 18 \mathrm{~ms}\right)$, intermediate if cue and goal were compatible in one reference frame but incompatible in the other reference frame $\left(\mathrm{I}_{\mathrm{E}} \mathrm{C}_{\mathrm{H}}\right.$ : $415 \pm 13 \mathrm{~ms} ; \mathrm{C}_{\mathrm{E}} \mathrm{I}_{\mathrm{H}}: 414 \pm 16 \mathrm{~ms}$ ), and slowest if cue and goal were incompatible in both reference frames $\left(\mathrm{I}_{\mathrm{E}} \mathrm{I}_{\mathrm{H}}\right.$ : $447 \pm 16 \mathrm{~ms}$ ). A repeated-measurement two-way ANOVA with factors eye compatibility and hand compatibility revealed a main effect for both, eye $(F(1,15)=22.558$, MSE $=1,163, P=0.0003)$ and hand $(F(1,15)=19.46$, $\mathrm{MSE}=1,263, P=0.0005)$ reference frame, with no interaction $(F=0.57, \mathrm{MSE}=1,545, P=0.46)$ in generalized trials. Standard trials were faster than generalized trials $\left(P<10^{-8}\right.$, paired $t$ test $)$.

Figure $3 \mathrm{~b}$ shows the average differences of all paired group comparisons. We conducted post hoc comparisons between all groups using paired $t$ tests $\left(\alpha_{\text {corr }}=0.0083\right.$ for $n=6$ multiple comparisons). The significances are indicated in Fig. 3b. Additionally, one can take from these post hoc comparisons that (a) the hand-compatibility effect, i.e. the RT difference between trials, which were compatible and trials, which were incompatible in a hand-centered reference frame, in eye-compatible trials $\left(\mathrm{C}_{\mathrm{E}} \mathrm{C}_{\mathrm{H}}\right.$ vs. $\mathrm{C}_{\mathrm{E}} \mathrm{I}_{\mathrm{H}}$; $47 \pm 14 \mathrm{~ms}$ ) is about equal to the eye-compatibility effect, i.e. the RT difference between trials which were compatible and trials which were incompatible in an eye-centered reference frame, in hand-compatible trials $\left(\mathrm{C}_{\mathrm{E}} \mathrm{C}_{\mathrm{H}}\right.$ vs. $\mathrm{I}_{\mathrm{E}} \mathrm{C}_{\mathrm{H}}$; $48 \pm 15 \mathrm{~ms}$ ), and (b) the hand-compatibility effect in eyeincompatible trials $\left(\mathrm{I}_{\mathrm{E}} \mathrm{C}_{\mathrm{H}}\right.$ vs. $\left.\mathrm{I}_{\mathrm{E}} \mathrm{I}_{\mathrm{H}} ; 32 \pm 13 \mathrm{~ms}\right)$ is about equal to the eye-compatibility effect in hand-incompatible trials $\left(\mathrm{C}_{\mathrm{E}} \mathrm{I}_{\mathrm{H}}\right.$ vs. $\mathrm{I}_{\mathrm{E}} \mathrm{I}_{\mathrm{H}} ; 33 \pm 10 \mathrm{~ms}$; Fig. 3b). The first and the second bar of Fig. 3b show that the difference between $\mathrm{I}_{\mathrm{E}} \mathrm{I}_{\mathrm{H}}$ and $\mathrm{C}_{\mathrm{E}} \mathrm{C}_{\mathrm{H}}$ trials is less for standard $(29 \pm 6 \mathrm{~ms})$ then for generalized $(80 \pm 14 \mathrm{~ms})$ trials $(P=0.0023$, paired $t$ test $)$. In summary, this means that RTs increase due to incompatibility in eye-reference frame and due to incompatibility in hand-reference frame and that both effects were about equally large.

Figure 4 shows the influences of different reference frames on SRC effects in MTs. The analysis is equivalent to 


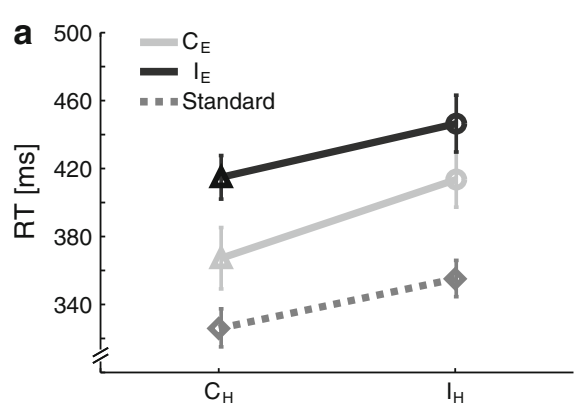

Fig. 3 Influence of SR compatibility in eye- and hand-reference frames on reach reaction times $(R T s)$. a Average (mean \pm SEM) RTs for the different combinations of compatibility/incompatibility in the hand-reference frame $\left(\mathrm{C}_{\mathrm{H}}\right.$ (triangles $) / \mathrm{I}_{\mathrm{H}}$ (circles)) and compatibility/ incompatibility in the eye-reference frame $\left(\mathrm{C}_{\mathrm{E}}\right.$ (light gray) $/ \mathrm{I}_{\mathrm{E}}($ black $\left.)\right)$. RTs in the standard trials are plotted separately (dashed, dark gray

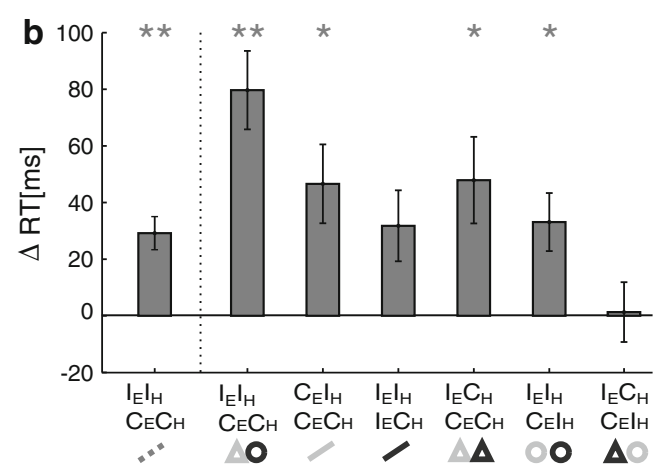

curve). Note that for the standard trials eye and hand compatibility are identical. b Average inter-subject difference between the compatible and incompatible trials in the standard condition (1 st bar) and between all possible combination of compatibility conditions in generalized trials. $* P<0.05$; $* * P<0.01$, paired $t$ test, Bonferroni corrected
Fig. 4 Influence of SR compatibility in eye- and hand-reference frames on reach movement times $(M T s)$. Conventions are the same as for Fig. 3

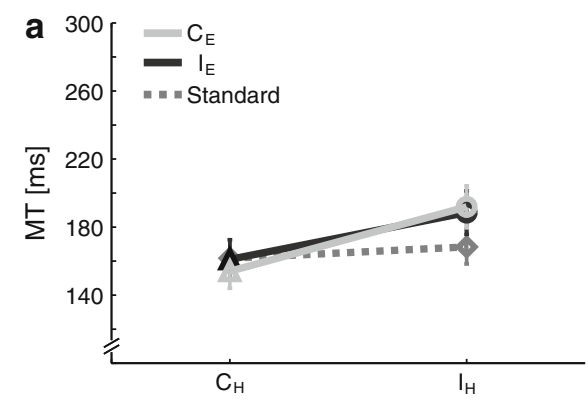

RTs in Fig. 3. MTs in standard pro-reaches $(162 \pm 11 \mathrm{~ms})$ were faster than in standard anti-reaches $(168 \pm 10 \mathrm{~ms}$; $P=0.042$, paired $t$ test; Fig. $4 \mathrm{a}$, dashed line; Fig. $4 \mathrm{~b}$, left bar). In generalized trials, the repeated-measurement twoway ANOVA with factors eye- and hand-reference frame revealed a main effect for hand-reference frame $(F(1,15)=$ 69.00, MSE $\left.=248, P<10^{-4}\right)$, but no effect for eye-reference frame $(F(1,15)=0.25$, MSE $=201, P=0.62)$, and no interaction $(F(1,15)=1.48, \mathrm{MSE}=288, P=0.24)$. Post hoc tests revealed that there was a hand-compatibility effect in both eye-compatible $\left(P<10^{-5}, \alpha_{\text {corr }}=0.0083\right)$ and eye-incompatible trials $(P=0.0003)$, while there was an eye-compatibility effect neither in hand-compatible $(P=0.14$, $)$ nor in handincompatible trials $(P=0.61)$.

The influence of SRC in eye- and hand-reference frame on EVs in the relevant horizontal $x$-dimension is shown in Fig. 5. Standard pro-trials show smaller EVs $(0.47 \pm$ $0.02 \mathrm{~cm}$ than standard anti-trials $(0.58 \pm 0.04 \mathrm{~cm}, P=$ 0.0017, paired $t$ test; Fig. 5a, dashed line; Fig. 5b, left bar). In generalized trials, the repeated-measurement two-way ANOVA showed a significant main effect of eye-reference frame $\left(F(1,15)=48.8, \mathrm{MSE}=0.003, P<10^{-4}\right)$, but no effect of hand-reference frame $(F(1,15)=1.19, \mathrm{MSE}=0.022$,
$P=0.29)$, and no interaction $(F(1,15)=1.34, \quad \mathrm{MSE}=$ $0.009, P=0.27)$. Post hoc tests revealed that there was no hand-compatibility effect in either eye-compatible $(P=0.077$, $\left.\alpha_{\text {corr }}=0.0083\right)$ or eye-incompatible trials $(P=0.84)$. There was an eye-compatibility effect in hand-compatible $(P=0.0005)$ but not hand-incompatible trials $(P=0.027)$. In our task design reach goal position varied only in the $x$-dimension. Accordingly, we did not see any effect of eyeor hand-reference frame on the EV in the $y$-dimension (data not shown).

\section{Constraint of eye movements}

The results described earlier were obtained while subjects were instructed to keep ocular fixation on the fixation spot, but without registering the actual eye movements. If subjects could not reliably follow the instruction of keeping their gaze fixed, but, for example, made many unvoluntary saccades toward the flashed spatial cue, then such saccades could have interfered with the reach initialization, and could thereby have confounded RT data.

We recorded 15 additional subjects (14 new, 1 from the previous sample) in the same task while constraining their 
Fig. 5 Influence of eye- and hand-reference frames on reach endpoint variability $(E V)$. Conventions are the same as in Fig. 3

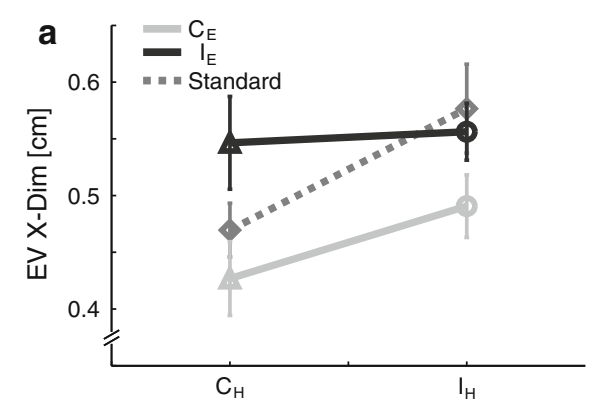

gaze in real time (see "Methods"). The control experiment turned out to be challenging to the subjects. On average across subjects, the success rate was $79 \pm 3 \%$ in standard pro-trials and $80 \pm 3 \%$ in standard anti-trials. The average success rate in the standard trials of the control experiment was lower than in the main experiment $(P=0.0055, t$ test, $N=16$ subjects (no constraint) and $N=15$ (with constraint)). The average RTs in the control experiment $(438 \pm 18 \mathrm{~ms})$ were higher by about $100 \mathrm{~ms}$ compared to the main experiment $\left(341 \pm 11 \mathrm{~ms} ; P<10^{-4}, t\right.$ test). The success rates in the generalized trials of the control experiment were $67 \pm 4 \%\left(\mathrm{C}_{\mathrm{E}} \mathrm{C}_{\mathrm{H}}\right), 67 \pm 3 \%\left(\mathrm{C}_{\mathrm{E}} \mathrm{I}_{\mathrm{H}}\right), 80 \pm 2 \%$ $\left(\mathrm{I}_{\mathrm{E}} \mathrm{C}_{\mathrm{H}}\right)$ and $71 \pm 3 \%\left(\mathrm{I}_{\mathrm{E}} \mathrm{I}_{\mathrm{H}}\right)$. This means that the task performance for the generalized conditions dropped significantly compared to the main experiment $\left(P<10^{-5}, t\right.$ test, $N=16$ subjects (no constraint) and $N=15$ (with constraint)). Whereas in the main dataset there was no significant success rate difference between standard and generalized conditions ( $88 \pm 1$ vs. $86 \pm 1 \%, P>0.05$, paired $t$ test), the success rate in generalized conditions of the control experiment was significantly lower $(69 \pm 3 \%)$ than in the standard conditions $\left(80 \pm 2 \% ; P<10^{-5}\right.$, paired $t$ test). This means that performance difficulties in the control experiment mainly affected the generalized conditions.

The poor overall performance in the control experiment did not allow systematic comparisons between the results of the control and main experiments (data not shown). We attribute the idiosyncratic and non-conclusive results of the control experiment to the overall increased task difficulty, as indicated by a significant drop in performance and strong increase in average RTs (cf. "Discussion”).

\section{Discussion}

We tested how reach RTs, MTs and EVs are influenced by the spatial compatibility between a visual cue and the associated motor-goal in an eye- and/or hand-centered frame of reference. Our results show that there was not a global consistent influence of one single reference frame on multiple movement parameters, but different reference frames affected different behavioral parameters in a specific way. RTs were influenced by both, SRC in eye- and hand-reference frame, whereas MTs were influenced only by SRC in the hand-reference frame, and EVs were influenced only by $\mathrm{SRC}$ in the eye-reference frame.

\section{Spatial reference frames for reach planning}

Previous neurophysiology studies showed different predominant frames of reference for reach targets in different brain areas. Spatial tuning properties of most neurons in the parietal reach region (PRR; Batista et al. 1999; Buneo et al. 2002; Pesaran et al. 2006) as well as human imaging data from the posterior parietal cortex (Medendorp et al. 2003) fit best with a representation of the reach target in an eyereference frame. Neurons in parietal area 5 as well as a subpopulation of neurons in PRR showed hand-centered tuning (Buneo et al. 2002; Chang and Snyder 2010). In premotor areas, tuning properties of most neurons are driven by a combination of eye, hand and target position for reaching, whereas some are purely hand-centered, and others are purely eye-centered (Batista et al. 2007; Pesaran et al. 2006). How the coding of neurons in different reference frames translates into overt behavior is not obvious and might depend on the specific task.

Previous psychophysical experiments analysed systematic reach errors suggesting reach goal encoding in an eyecentered reference frame (Beurze et al. 2006; Henriques et al. 1998; Sorrento and Henriques 2008). Other experiments, which also analysed systematic reach errors, provided evidence for a hand-centered representation of reach goals (Bock and Eckmiller 1986; Gordon et al. 1994), or a combination of different reference frames (McGuire and Sabes 2009). This means, previous studies based on systematic reach errors showed idiosyncratic results. The diversity of findings could be due to the fact that reach errors can be expected to be influenced by different processes of motor planning and control or different sources of sensory input were differently weighted (McGuire and Sabes 2009).

Other studies have investigated the influence of reference frames on SR compatibility effects in reaction time 
(Lamberts et al. 1992; Nicoletti and Umilta 1989; Umilta and Liotti 1987). Lamberts et al. (1992) found joint compatibility effects of visual hemifield and relative position of two stimuli, in a task in which the response required buttonpresses of either the ipsi- or contralateral hand. In contrast to our study, this previous study could not compare the dissociated effects of an eye- and hand-reference frame, which was mostly investigated in neurophysiological studies. The reason for the inaccessibility of the hand-reference frame in the Lamberts study was the use of a dissociated workspace for visual cue and bimanual button-press responses (Lamberts et al. 1992), which is known to influence compatibility effects (Stins and Michaels 2000). Our results confirmed the influence of an eye-reference frame on RTs, and additionally show an influence of the hand-reference frame. Moreover, we could show distinct effects on other movement parameters, as discussed in the following paragraph.

\section{Kinematic reach parameters}

In contrast to RTs, MTs in our experiment were only influenced by compatibility in the hand-reference frame. The fact that MTs reflect parameters of movement planning and control might account for this, and is consistent with the notion, that hand-reference frames gain increasing importance the closer a brain structure is to the motor output (Batista et al. 2007; Pesaran et al. 2006).

EVs showed a significant effect of eye- but not of handreference frame. This effect was mainly induced by a reduced $\mathrm{EV}$ in trials with SR compatibility in both eye and hand-reference frame (Fig. 5a). Eye- and hand-compatible trials are characterized by the fact that cue and motor-goal were physically identical (same screen position). Despite the spatial cue being only briefly flashed (s. Methods), this might have led to a certain degree of visual guidance with reduced variability in movement trajectories, whereas in the other generalized task conditions the reach goal had to be spatially inferred, without the possibility of direct visual guidance.

\section{Spatial stimulus-response compatibility}

In the previous section, we interpreted and discussed our results in terms of eye- and hand-reference frames and their influence on SR compatibility effects. SR compatibility was defined as left/right compatibility of cue and motor-goal directions. However, there might be a more parsimonious explanation for the observed RT differences. RTs in the main experiment can be grouped to three different levels (Fig. 3). There was no difference between $\mathrm{C}_{\mathrm{E}} \mathrm{I}_{\mathrm{H}}$ reaches and $\mathrm{I}_{\mathrm{E}} \mathrm{C}_{\mathrm{H}}$ reaches $\left(P=0.9\right.$, paired $t$ test, $\left.\alpha_{\text {corr }}=0.0083\right)$. These three levels of RTs correlate with the distance between spatial cue and reach goal. A previous study (Stins and Michaels 2000) showed that the distance between cue and target can indeed influence RTs. Subjects are faster the closer the cue and target were together. In our task design, we cannot differentiate between the possibilities that RTs are explained by the combined compatibility in eye- and hand-reference frame, or by the distance between cue and reach goal.

Unlike RTs, MTs and EVs cannot be explained by the same dependency of absolute distance between cue and reach goal, since neither movement parameter scaled with this absolute distance. But the MT and EV results could possibly be explained by the compatibility of cue and goal eccentricity, i.e. the distance of the cue and the reach goal from the eye- or hand-fixation position (as depicted by the length of the arrows in Fig. 1b). According to this alternative view, compatibility of the direction of cue and reach goal would be irrelevant. Instead, reaches which are faster and more precise if cue and reach goal are compatible in eye eccentricity, i.e. if cue and goal are at the same distance from the eye-fixation, would indicate an influence of the eye-reference frame, Correspondingly, a compatibility effect of hand eccentricity would be taken as indication for an influence of the hand-reference frame. With this interpretation, RTs would have been determined by the compatibility of cue and goal eccentricity in an eye- and hand-reference frame. MTs would have been determined by the compatibility of cue and goal eccentricity in an eye-centered reference frame only. The latter means that reaches were faster if the reach goal position had the same distance from eye fixation as the cue compared to conditions in which the reach goal had a larger distance from eye fixation, despite identical hand eccentricity of the goal (=reach amplitude) in both conditions. EVs would have been determined by cue and reach goal eccentricity in a hand-centered reference frame. EVs were smaller if the cue had the same distance to the hand-fixation stimulus as the reach goal compared to conditions in which the cue had a larger distance from the hand-fixation stimulus, despite identical eye eccentricity of the cue in both conditions. We consider the possibility that MTs and EVs are explained by the compatibility of cue and goal eccentricities less plausible, since at least for the EVs it seems counter intuitive that they should be influenced by the distance of the cue from the hand-fixation stimulus independent of reach amplitude.

\section{Effect of eye movements}

In the control experiment, we wanted to test in how far involuntary saccades in the main experiment could have confounded our results. In standard trials of the control experiment, subjects performed similarly well as in the main experiment, and we found qualitatively the same results. However, results across subjects in the generalized conditions of the control experiment were rather idiosyncratic. We 
rule out that the RT results of the main experiment are explained by involuntary saccades aimed at the flashed spatial cue (or a following reorientation saccade toward the goal). If this was the case then we would have to expect that ocular fixation breaks mostly happened during or briefly after the presentation of the spatial cue, which was only the case for less than $19 \%$ of all ocular fixation breaks (3.7\% of all trials). Also, the standard pro-reaches and generalized anti-reaches denote trials with physically identical reaches, in which the cue was identical to the reach goal position and at the same distance from the fixation position. Yet, RTs in the main experiment were significantly different between these two conditions ( $P=0.0054$, paired $t$ test), again arguing against an effect induced by involuntary eye movements. Instead, we attribute the idiosyncratic results of the control experiment to the overall increased task difficulty, as indicated by significant drop in task performance and strongly increased overall reaction times (see "Results").

\section{Conclusions}

Different movement parameters, like reaction times, movement times, and endpoint variability, each reflect the stages of planning and control of a motor act to a different degree. We could show that eye- and hand-frames of reference have selective effects on the different movement parameters during goaldirected reaching. Hence, our results imply that eye- and hand-reference frames have distinct effects on the different stages of planning and control. In this sense, our results denote a psychophysical manifestation of the different observed reference frames at the different stages of neuronal processing, which putatively underlie different phases of overt motor behavior. During the planning stage, which we argue should be best reflected in the SRC effects on RTs, we found a twofold influence of eye- and hand-reference frame, without interaction, reminiscent of mixed reference frames of neurons in the parietal and frontal reach related sensorimotor areas.

Acknowledgments We thank Sadeem Jawhar and Constanze Lenschow for help in data collection, and Sina Plümer and Ludwig Ehrenreich for administrative and technical support. This work was supported by the Federal Ministry for Education and Research (BMBF, Germany) grants 01GQ0433 and 01GQ0814.

Open Access This article is distributed under the terms of the Creative Commons Attribution Noncommercial License which permits any noncommercial use, distribution, and reproduction in any medium, provided the original author(s) and source are credited.

\section{References}

Ariff G, Donchin O, Nanayakkara T, Shadmehr R (2002) A real-time state predictor in motor control: study of saccadic eye movements during unseen reaching movements. J Neurosci 22:7721-7729
Batista AP, Buneo CA, Snyder LH, Andersen RA (1999) Reach plans in eye-centered coordinates. Science 285:257-260

Batista AP, Santhanam G, Yu BM, Ryu SI, Afshar A, Shenoy KV (2007) Reference frames for reach planning in macaque dorsal premotor cortex. J Neurophysiol 98:966-983

Beurze SM, Van Pelt S, Medendorp WP (2006) Behavioral reference frames for planning human reaching movements. J Neurophysiol 96:352-362

Bock O, Eckmiller R (1986) Goal-directed arm movements in absence of visual guidance-evidence for amplitude rather than position control. Exp Brain Res 62:451-458

Buneo CA, Andersen RA (2006) The posterior parietal cortex: sensorimotor interface for the planning and online control of visually guided movements. Neuropsychologia 44:25942606

Buneo CA, Jarvis MR, Batista AP, Andersen RA (2002) Direct visuomotor transformations for reaching. Nature 416:632-636

Chang SWC, Snyder LH (2010) Idiosyncratic and systematic aspects of spatial representations in the macaque parietal cortex. PNAS 107:7951-7956

Crammond DJ, Kalaska JF (1994) Modulation of preparatory neuronal activity in dorsal premotor cortex due to stimulus-response compatibility. J Neurophysiol 71:1281-1284

de Jong R (1995) Strategical determinants of compatibility effects with task uncertainty. Acta Psychol 88:187-207

Duncan J (1977) Response selection errors in spatial choice reaction tasks. Q J Exp Psychol 29:415-423

Everling S, Dorris MC, Munoz DP (1998) Reflex suppression in the anti-saccade task is dependent on prestimulus neural processes. J Neurophysiol 80:1584-1589

Fischer B, Weber H (1992) Characteristics of antisaccades in man. Exp Brain Res 89:415-424

Fitts PM, Deininger RL (1954) S-R compatibility: correspondence among paired elements within stimulus and response codes. J Exp Psychol 48:483-492

Fitts PM, Seeger CM (1953) S R compatibility—spatial characteristics of stimulus and response codes. J Exp Psychol 46:199-210

Gail A, Andersen RA (2006) Neural dynamics in monkey parietal reach region reflect context-specific sensorimotor transformations. J Neurosci 26:9376-9384

Georgopoulos AP, Lurito JT, Petrides M, Schwartz AB, Massey JT (1989) Mental rotation of the neuronal population vector. Science 243:234-236

Gordon J, Ghilardi MF, Ghez C (1994) Accuracy of planar reaching movements. I. Independence of direction and extent variability. Exp Brain Res 99:97-111

Hallett PE (1978) Primary and secondary saccades to goals defined by instructions. Vision Res 18:1279-1296

Henriques DYP, Klier EM, Smith MA, Lowy D, Crawford JD (1998) Gaze-centered remapping of remembered visual space in an openloop pointing task. J Neurosci 18:1583-1594

Hommel B (1996) No prevalence of right-left over top-bottom spatial codes. Percept Psychophys 58:102-110

Khan AZ, Pisella L, Rossetti Y, Vighetto A, Crawford JD (2005) Impairment of gaze-centered updating of reach targets in bilateral parietal-occipital damaged patients. Cereb Cortex 15:15471560

Lamberts K, Tavernier G, d'Ydewalle G (1992) Effects of multiple reference points in spatial stimulus-response compatibility. Acta Psychol 79:115-130

McGuire LMM, Sabes PN (2009) Sensory transformations and the use of multiple reference frames for reach planning. Nat Neurosci 12:1056-1061

Medendorp WP, Goltz HC, Vilis T, Crawford JD (2003) Gaze-centered updating of visual space in human parietal cortex. J Neurosci 23:6209-6214 
Morin RE, Grant DA (1955) Learning and Performance on a key-pressing task as function of the degree of spatial stimulusresponse correspondence. J Exp Psychol 49:39-47

Nicoletti R, Umilta C (1984) Right-left prevalence in spatial compatibility. Percept Psychophys 35:333-343

Nicoletti R, Umilta C (1989) Splitting visual space with attention. J Exp Psychol Hum Percept Perform 15:164-169

Pesaran B, Nelson MJ, Andersen RA (2006) Dorsal premotor neurons encode the relative position of the hand, eye, and goal during reach planning. Neuron 51:125-134

Proctor RW, Vu KP (2002) Mixing location-irrelevant and locationrelevant trials: influence of stimulus mode on spatial compatibility effects. Mem Cognit 30:281-293
Shaffer LH (1965) Choice reaction with variable S-R mapping. J Exp Psychol 70:284-288

Sorrento GU, Henriques DYP (2008) Reference frame conversions for repeated arm movements. J Neurophysiol 99:2968-2984

Stins JF, Michaels CF (2000) Stimulus-response compatibility for absolute and relative spatial correspondence in reaching and in button pressing. Q J Exp Psychol Sect A Hum Exp Psychol 53:569-589

Umilta C, Liotti M (1987) Egocentric and relative spatial codes in S-R compatibility. Psychol Res Psychologische Forschung 49:81-90

Zhang M, Barash S (2000) Neuronal switching of sensorimotor transformations for antisaccades. Nature 408:971-975 


\section{Motor learning and motor adaptation}

\section{III.I. Motor learning}

To survive in an environment which is constantly changing during their lifetime it is not sufficient for animals and humans to rely on pre-defined motor programs or reflexes. To be able to deal with changes in the world they have to expand their motor repertoire to develop new motor programs. Additionally those motor programs have to be constantly adapted and changed in such a way that the original -best- motor performance can be maintained. The two versions of motor learning, namely skill acquisition and motor adaptation differ in the following points. In skill acquisition the motor system learns a new skill and therefore extends the performance of its borders. For example, if one learns to ride a bicycle one has, after some practice, accomplished the ability of a new form of locomotion without loosing the ability to walk. Skill acquisition seems to depend on the given circumstances of the task and the way how one accomplishes these tasks can vary largely between individuals. In motor adaptation, in contrast, one does not expand the capabilities of the motor system. Motor adaptation becomes important after changes in the environment, which reduces one's original motor performance. Motor adaptation allows the system to react to those changes and brings back the performance of the motor system to its original level. If, for example, somebody changes the speed settings of ones computer mouse, in the beginning one will not be able to use the cursor precisely and it will miss the target one aimed at. After a while, however, one adapts to the new settings and can handle the mouse with the new settings as precisely as before. In contrast to skill acquisition in this case one has not expanded one's motor program but overwritten an existing program. This becomes obvious if one switches back the settings of the computer mouse to the old settings. In this case one will not be precise anymore and again miss the targets, even though one had been able to handle the same settings before. But this old motor program got overwritten during the adaptation to the new settings. This requirement for a de-adaptation, after the adaptation, in order to go back to the original settings, is the main difference to skill acquisition. In experiments, which study motor adaptation, this property manifests itself in so called after-effects, which causes an error in performance which is in the opposite direction to the error cause by the original distortion. 


\section{III.II. Reach adaptation}

In reach adaptation experiments, adaptation usually was elicited in three different ways. First, experimenters induced a mismatch between different sensory modalities e.g. with prisms, which distort the visual input. Second, in so-called visuomotor rotation tasks the trajectory of a given motor command is distorted. Normally in these experiments the visual feedback of one's hand is replaced by a computer cursor. In the baseline condition the cursor represents the original hand trajectory. However, during the distortion the mapping between the hand and the cursor changed e.g. if one moves its hand straight up, the cursor on the screen would move to the right. Third, adaptation can be elicited by applying an unexpected force to ones hand during the movement, which then causes a deviation of the hand from its normal trajectory. In all three cases, one adapts to the perturbations and subjects, after a while, are able to perform precise, straight reaches again.

Lesion studies (Weiner et al., 1983; Martin et al., 1996; Baizer et al., 1999; Kurata and Hoshi, 1999) as well as imaging studies (Clower et al., 1996; Desmurget et al., 1998; Diedrichsen et al., 2005) showed an involvement of the cerebellum, the premotor cortex and the posterior parietal cortex during adaptation. Other studies with electrophysiological studies in monkeys show changes in tuning properties of neurons in M1, which is the main output to the spinal cord for movement generation (Wise et al., 1998; Li et al., 2001; Paz et al., 2003; Mandelblat-Cerf et al., 2009; Arce et al., 2010b).

Behaviorally, the strength of the effect of reach adaptation can be influenced by the form of the given feedback. Adaptation effects are stronger if the feedback is provided by one's own hand in contrast to a cursor feedback (Clower and Boussaoud, 2000; Norris et al., 2001). Another study showed that the size of prism-adaptation effects decreases if the feedback signal of the hand is delayed after reaching the target position (Kitazawa et al., 1995). Also, implicit strategies of the subjects do not prevent adaptation. Even if subjects use cognitive strategies to counterbalance the distortion (in a visuomotor rotation task) the motor system still adapts to the mismatch between visual input/feedback and the expected visual output of the issued motor command, which in the given situation causes a decrease in performance due to adaptation effects (Mazzoni and Krakauer, 2006). 


\section{III.III. Generalization}

If one adapts to a certain distortion in a specific situation, how does this transfer to other situations? Are there still adaptation effects if the arm is moved to a different position or if the reach aims at a different target? Several studies dealt with the question of generalization in reach adaptation, since the pattern of generalization allows conclusions about the properties of the underlying neuronal units. In tasks which induce a rotational distortion, the adaptation effect is maximal for the direction in which the subject performed reaches during the exposure of the distortion. However for targets near the adapted direction subjects still showed an adaptation effect but the size of this effect decreased with increasing distance from the target (Imamizu et al., 1995; Pine et al., 1996; Krakauer et al., 2000; Donchin et al., 2003; Thoroughman and Taylor, 2005; Mattar and Ostry, 2007; Mattar and Ostry, 2010). In contrast to this partial generalization to different target directions there seems to be a more complete generalization to different gains. If subjects were adapted with a distortion in which the translation of the arm movement to a respective cursor movement had a altered gain factor, i.e. the cursor moved faster/further or slower/nearer, this adaptation transferred to different target distances as well as to different directions (Bock, 1992; Krakauer et al., 2000). It was shown that reach adaptation generalizes partly to different postures of the arm (Baraduc and Wolpert, 2002) and to different positions in workspace as long as the target vector (difference between starting position and the target) and movement vector (starting position and the reach endpoint) are kept constant (Wang and Sainburg, 2005). It seems that at least partly, the broadness of the generalization function depends on whether there is a simple rule which can be learned in one situation and then easily transferred to other situations. For example for gain adaptation there is a linear relationship between peak velocity and target distance, which is approximately constant for the whole workspace. In contrast, the relationship for directional generalization can not be estimated from a single input-output coupling (Krakauer et al., 2000).

In a psychophysical experiment, we used a generalization paradigm to, first, examine whether the adaptation is coded in a Cartesian or a polar reference frame and, second, test if the direction of the adaptation effect causes an asymmetry in the generalization. 


\section{Asymmetric generalization in adaptation to target displacement errors}

Stephanie Westendorff ${ }^{1,2}$, Shenbing Kuang ${ }^{1}$, Bahareh Taghizadeh ${ }^{1}$, Opher Donchin ${ }^{3}$, Alexander Gail ${ }^{1,2}$

1. German Primate Center, Kellnerweg 4, 37077 Göttingen, Germany

2. Bernstein Center for Computational Neuroscience Göttingen

3. Department of Biomedical Engineering and Zlotowski Center for Neuroscience, Ben-Gurion University of the Negev, Beer Sheva, Israel 


\begin{abstract}
Adaptation is crucial for the sensorimotor system to compensate systematic changes in the environment. In visuomotor tasks, artificially perturbed visual feedback about hand position leads to movement adaptation, which could be driven by prediction errors about sensory hand representations or the induced target error. While the first is mostly believed to be decisive for adaptation, we here demonstrate and characterize reach adaptation in a novel visuomotor task based on target error alone. A sudden target displacement ('jump') induced reach errors, when perception of the jump was suppressed by a simultaneous saccade to a separate target, while hand perception was unperturbed. Generalization was characterized by two findings. First, reach endpoints to unperturbed targets were off-set in a direction parallel to the jump, indicating adaptation of endpoint position rather than reach direction. Second, generalization was asymmetric, with larger adaptation for targets in the direction of the target jump. Our results demonstrate that reach adaptation can be driven by target error alone. The asymmetric generalization pattern suggests neural adaptation mechanisms different from visuomotor rotation tasks, which typically generalize symmetrically. A recurrent neural network model which we previously used for simulating context-specific sensorimotor transformations reproduces the asymmetry observed in the human behavioral data, but only under specific conditions. The model thereby puts constraints on the possible underlying neural mechanisms of adaptation in our paradigm and makes testable predictions about selective widening and narrowing of the tuning in sensorimotor neurons which are tuned for positions near the adapted target position.
\end{abstract}




\section{Introduction}

Our sensorimotor system needs to adapt to environmental changes to sustain proper performance. Depending on which aspect of a movement is perturbed, adaptation may serve the purpose of reducing the offset between reach endpoint and target ('target error', (Diedrichsen et al., 2005; Magescas et al., 2009; Cameron et al., 2011), readjusting forward model predictions which no longer match the actual sensory feedback (Shadmehr and Krakauer, 2008), or both. To understand the sensorimotor system, it is essential to know how different functional perturbations map onto changes at different levels of the sensorimotor process.

In the widely used visuomotor rotation task (Bedford, 1989; Bock, 1992; Kitazawa et al., 1995; Imamizu et al., 1995; Pine et al., 1996; Martin et al., 1996; Ghahramani et al., 1996; Wise et al., 1998; Vetter et al., 1999; Krakauer et al., 2000; Paz et al., 2003; Krakauer et al., 2004; Redding and Wallace, 2006) kinematic adaptation is elicited by a perturbed visual feedback (cursor) about hand position. This perturbation leads to a mismatch between the learned visual and proprioceptive feedback of hand position, and to prediction errors in the sense that a familiar motor command leads to unexpected sensory consequences. When the perturbation can not be compensated during the course of the movement then also target errors occur. Unless one can isolate a single source of adaptation, it is difficult to attribute adaptive behavioral changes to an individual cause. While sensory prediction error alone has been shown to induce adaptation (Mazzoni and Krakauer, 2006), consistent noticeable target error alone failed to induce adaptation in previous studies (Diedrichsen et al., 2005). In an attempt to isolate target error as an effective source of adaptation, we tested if clearly noticeable target errors despite unimpaired hand localization can induce implicit adaptation in reaching.

In our novel target-jump paradigm subjects directly saw their own hand, and therefore knew that perturbation of the seen hand position was impossible. Saccadic suppression rendered a peri-movement target jump invisible, while the resulting target error was obvious to the subjects. We tested if adaptation to target error alone would show similar transfer of adaptation to neutral target positions (generalization) as other forms of visuomotor adaptation, or if generalization characteristics suggested adaptation at a different level of sensorimotor processing or with a different 
underlying neural mechanisms. We tested if reach directions (Bock, 1992; Krakauer et al., 2000) or reach endpoints (Ghahramani et al., 1996) would be adapted, and if generalization patterns were symmetric, as observed in previous visuomotor rotation tasks (Pine et al., 1996; Krakauer et al., 2000). With a recurrent neural network, which was previously built to simulate context-specific visuomotor associations (Brozovic et al., 2007), we could simulate the target-jump adaptation of our experiment and reproduce our observed asymmetry in generalization. Our psychophysical and modelling results suggest that target jump induces implicit adaptation of visuospatial motor goal representations, and, complementary to the case of symmetric generalization (Tanaka et al., 2009), that neurons in visuomotor layers should change their tuning properties during adaptation. 


\section{Methods}

\section{Subjects}

In total, 47 subjects (36 females, 20 to 58 years) participated in the experiment. Detailed written instructions were given to the subjects before the experiment. In a training session, a few minutes before the recording session, subjects could practice the control task without target jump and ask any question not resolved by the written instructions. All subjects were right handed with normal or corrected-to-normal vision and were naïve with respect to the objective of the study. Subjects were not informed about the target jump that would be introduced. Experiments adhered to the principles of the Declaration of Helsinki.

\section{Task design}

Subjects simultaneously had to reach and saccade to two separate targets in the opposite hemifield on a frontoparallel screen. Subjects did free reaches towards the touch screen and could see their own hand during the whole experiment, i.e. they had direct visual feedback about the hand position during the reach. Saccadic suppression allowed repositioning of the reach target (target jump), without subjects noticing. Saccade targets were chosen to be opposite to the reach target, i.e. spatially separate to avoid interference of saccadic and reach adaptation (Bekkering et al., 1995; De Graaf et al., 1995). When interviewed after completion of the experiment, most subjects (32/46) reported not to have noticed any target perturbation (see below for subject selection).

The task consisted of three different trial types: standard, jump, and probe trials (Fig. 1A, B). In all three trial types subjects had to perform center-out reaches and saccades. In all trial types the peripheral saccade target remained visible and stationary. In standard trials the peripheral reach target remained visible and stationary until the trial was finished. In jump trials the reach target remained visible but was re-located to a new position during saccade execution. In probe trials the reach target disappeared during saccade execution and reaches had to be executed to the remembered target position. 
Saccade and reach targets were only varied in their direction relative to central eye and hand fixation, and appeared at a fixed eccentricity of $12 \mathrm{~cm}\left(=17.2^{\circ}\right.$ visual angle (VA) at $40 \mathrm{~cm}$ screen distance), unless stated otherwise. In all trial types the direction of the saccade target randomly alternated between two fixed positions at $195^{\circ}$ and $285^{\circ}$ from trial to trial. In standard and jump trials the initial peripheral reach target appeared at a fixed direction of $60^{\circ}$ (adapted direction, AD). In probe trials the reach target position varied (see below) in order to measure the generalization of adaptation and to establish a baseline performance before adaptation. To balance potential asymmetries of the workspace, we tested a counter-clockwise jump (CCW; N=30 subjects) and a clockwise jump (CW; $\mathrm{N}=17 ; 4$ subjects in both conditions). The $\mathrm{CW}$ and $\mathrm{CCW}$ experiments partially differ in the number of trials in the different experimental periods (see below) to optimize parameter settings in the $\mathrm{CW}$ experiment, which was conducted after the CCW experiment. The differences in trial numbers did not affect the results or the conclusions.

\section{Task conditions}

For each subject the experiment consisted of a sequence of task conditions (Fig. 1C). The control task was used to measure baseline performance. Subjects first conducted 10 standard trials to the AD. The standard trials were followed by 100(120) probe trials to $10(12)$ screen positions in the $\mathrm{CCW}(\mathrm{CW})$ experiment. Seven directional targets were located at $15,35,50,60,70,85$ and $105^{\circ}$, i.e. at $-45,-25,-10,0,10,25$, and $45^{\circ}$ relative to $\mathrm{AD}$ (Fig. 1D). An additional 3(5) radial targets were tested at the direction of the $\mathrm{AD}$ in the $\mathrm{CCW}(\mathrm{CW})$ experiment, which had an eccentricity of 7,10 , 14,17 and $20.5 \mathrm{~cm}$, i.e. $-5,-2,2,5$, and $7.5 \mathrm{~cm}$ relative to the adapted target. The order of appearance of the target positions was randomized and different for each subject. On average each target appeared 10 times. 
A

Standard

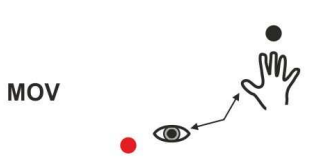

B

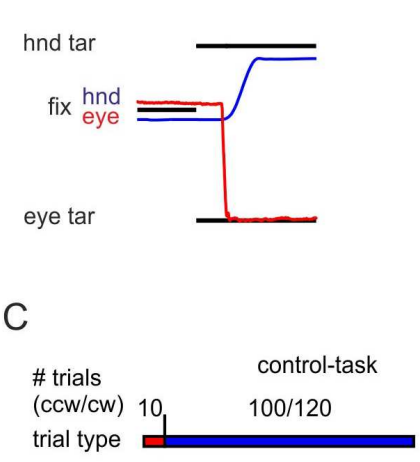

Target Jump
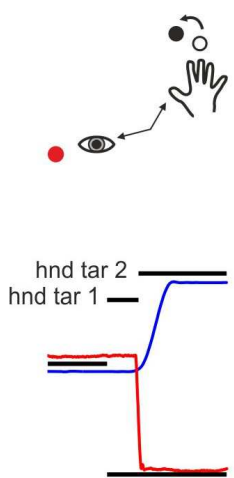

Probe
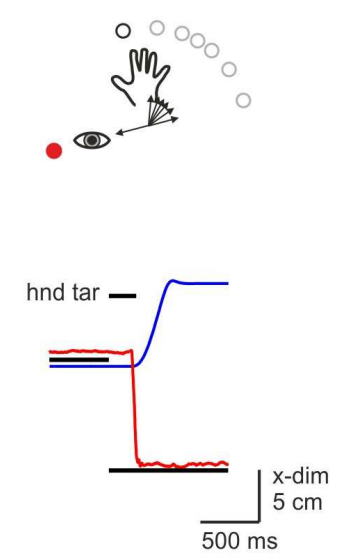

D

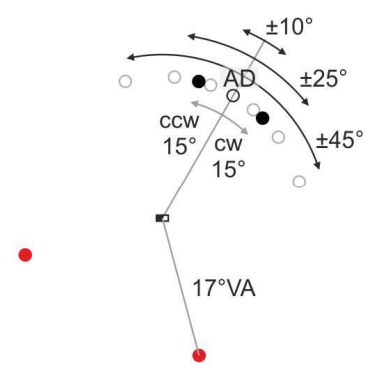

Figure 1 Target jump task with saccadic suppression. A: Trial types. The presentation of reach (black) and saccade (red) targets for standard, target jump and probe trials are shown during the time of movement. Filled symbols depict visible targets, black open symbols depict previous reach target positions, gray symbols depict alternative probe target positions in probe trials. For saccade targets only one of the two alternative positions is shown. B: Example behavior during standard, target jump and probe trials. Black lines represent duration and position of eye and hand targets. Red lines depict eye and blue lines hand positions. Note that positions are shown only in the horizontal dimension to be able to show the tangential target jump as well as the radial centerout movements of hand and eye within one dimension as a function of time. C: Sequence of task conditions. Red encodes standard trials, green target jump trials and blue probe trials. Hatching indicates randomly interleaved trial types. D: Spatial layout of the generalization task. Eye and hand fixation stimuli are shown as black and white squares, possible probe target positions are shown as gray open circles, possible saccade targets are shown as red circles. The target for the adapted direction (AD) is shown as open black circle. Target positions after the clockwise (CW) and counter-clockwise (CCW) jump of the target from the position at the adapted direction (AD) are shown as black filled circles.

The generalization task started with 10 standard trials to the AD (pre-exposure period) and ended with 15(20) standard trials to the AD in the $\mathrm{CCW}(\mathrm{CW})$ experiment (postexposure period). Between these trials, 40 jump trials were conducted in which the reach target was re-positioned to $75^{\circ}\left(\mathrm{CCW}\right.$ jump of $\left.15^{\circ}\right)$ or to $45^{\circ}\left(\mathrm{CW}\right.$ jump of $\left.15^{\circ}\right)$, 
while keeping the eccentricity constant (exposure period). Jumps of $15^{\circ}$ direction change corresponded to a $3.1 \mathrm{~cm}$ target offset on the screen.

To test generalization, subjects conducted probe trials to all target positions (generalization period) in between the exposure and post-exposure periods. The probe target positions matched the positions in the control condition and were presented in a random order. In the generalization period, probe trials appeared with a probability of $60 \%$ (average 108/180 trials) in the CCW experiment, and with 40\% (120/300) in the $\mathrm{CW}$ experiment. The remaining trials of the generalization period were jump trials, which served as refresher to maintain adaptation.

\section{Behavioral control and recording}

Visual instruction stimuli were presented on a LCD screen (19" ViewSonic VX922) in the frontoparallel plane. Subjects initiated a trial by fixating a small, red fixation spot $(0.5 \mathrm{~cm}$ square $)$ and touching an adjacent, gray hand fixation spot $(0.5 \mathrm{~cm}$ square) in the center of the screen (fixation period). After a variable delay (0.5 to 1.0 s) the fixation stimuli disappeared ('go-signal'), while simultaneously the reach target (gray, $0.5 \mathrm{~cm}$ radius) and the saccade target (red, $0.5 \mathrm{~cm}$ radius) appeared. Hand fixation had to be continuously touched within $2.5 \mathrm{~cm}\left(3.6^{\circ} \mathrm{VA}\right)$ and reach targets had to be continuously touched within $5 \mathrm{~cm}\left(7^{\circ} \mathrm{VA}\right)$ tolerance radius. Reach target tolerance was chosen to be relatively large such that it would not impose constraints onto adaptation or generalization effects. Eye fixation and saccade targets had to be continuously fixated within a tolerance window of $3 \mathrm{~cm}\left(4.3^{\circ} \mathrm{VA}\right)$ radius. Fixation breaks of either hand or eye immediately aborted the trial. Subjects had $700 \mathrm{~ms}$ to react to the appearance of the eye and hand targets by initiating a saccade. Saccade initiation was defined as the time when the gaze signal exited the eye-tolerance window. The eye had to leave the tolerance window in a direction of $\pm 45^{\circ}$ of the direction of the saccade target, otherwise the trial was aborted. The target jump was triggered 24-40 ms after saccade initiation (depending on when the saccade occurred relative to the refresh cycle of the screen). After saccade initiation subjects had to position gaze and hand within the tolerance window of the respective targets within $500 \mathrm{~ms}$. Gaze and hand had to stay within the tolerance windows for additional 500 ms to successfully finish the trial. 
Custom-written visual display software $(\mathrm{C}++)$ was controlled via a real-time LabView control program running on a PXI real-time computer (National Instruments). Eye and hand movements were monitored in real-time. Reach endpoints were registered on a touch screen (IntelliTouch, ELO Systems, CA, USA) mounted in front of the visual display. In 10 subjects of the CW experiment, we additionally measured hand trajectories at $200 \mathrm{~Hz}$ with a 3D optical motion tracking device (4300 Visualeyez, PTI Systems). One marker was attached to the nail of the index finger of the preferred hand, which was used by all subjects to touch the screen. Motion tracking data were smoothed with a 6th-order Butterworth filter (10 Hz cut-off frequency). Gaze positions were registered with an infrared eye tracking device at $500 \mathrm{~Hz}$ (SMI Sensomotoric Instruments, Germany). Subjects used a chinrest to minimize head movements.

\section{BEHAVIOR ANALYSIS}

\section{Time course of adaptation}

We fitted the directional reach errors during the exposure and post-exposure with an exponential function over successive trials $t$ to quantify incremental changes in the reach endpoint positions due to adaptation or re-adaptation (Fig. 2):

$$
\operatorname{dev}(t)=o f f s e t+a e^{-\varepsilon a}
$$

were $\varepsilon$ defines the learning rate, offset defines the asymptotic reach error and $a$ defines the scale. Directional reach errors were defined as the angular deviation of the direction of the reach from the direction of the target. Directions were defined as center-out direction from the fixation stimulus to the target/reach endpoint. To compensate idiosyncratic biases, we subtracted the mean directional reach error in the pre-exposure period from the directional reach error in each trial for each individual subject before averaging across subjects.

\section{Translational versus rotational generalization}

To quantify the amount of generalization properly, we first needed to test in which way adaptation transferred to neutral probe targets. For this we analyzed the $2 \mathrm{D}$ reach endpoint positions, not only the reach directions as above. We compared the error vector at the $\mathrm{AD}$ to the error vectors at the different directional probe targets. The error vector for each target was defined as the difference vector between the mean 
reach endpoints in the probe trials of the control condition and the probe trials in the generalization period of the test condition. This means, the direction of error vectors is measured relative to the baseline reach endpoints which correspond to each probe target. Error vectors were measured separately for each subject.

Two hypotheses concerning the directional aspect of the generalization were tested. According to the translation hypothesis, the direction of the error vector is independent of the probe target position (red arrows in Fig. 3). Any spatial profile of the generalization pattern across the work space would affect only the length of the error vector, not its direction. Such generalization would suggest a transfer of the 2D shift in the Cartesian endpoint position due to adaptation. According to the rotation hypothesis, the error vector is always tangential to the probe target direction (blue arrows, Fig. 3). The generalization pattern across the work space would affect the length and direction of the error vector simultaneously in a specific way. Such generalization would suggest a transfer of only the 1D direction of the adapted reach endpoints. Note, the task design does not constrain the subjects. The jump can be either interpreted as a positional shift or a directional change of the target, hence either generalization pattern is possible. We included only the directional probe targets, not the radial probe targets, in this analysis, since only targets with different reach direction than the $\mathrm{AD}$ allowed us to differentiate between the translation and the rotation hypothesis.

The measured error vector at the adapted target in individual subjects might not perfectly match the physical displacement of the target (the size and direction of the jump). We accounted for inter-individual differences in adaptation when testing for the alternative translation and rotation hypotheses. We used the error vector at the adapted target (Fig. 3A, $\vec{e}_{\mathrm{AD}}$ ) as reference for predicting the error vectors at the probe targets according to the translation and the rotation hypotheses. The predicted error vectors for the translation hypothesis $\left(\vec{t}_{T}\right)$ and for the rotation hypothesis $\left(\vec{r}_{T}\right)$ at each probe target $\mathrm{T}$ were defined as:

$$
\begin{aligned}
& \vec{t}_{T}=\vec{e}_{A D} \\
& \vec{r}_{T}=R\left(\delta_{T}\right) \vec{e}_{A D}=R\left(\delta_{T}\right) \vec{t}_{T}
\end{aligned}
$$


were $\mathrm{R}$ is a 2-D rotation matrix, and $\delta_{\mathrm{T}}$ equals the angular difference between the mean reach endpoint at the $\mathrm{AD}$ and the mean reach endpoint at the respective probe target in the control condition (Fig. 3A).

We compared the direction of the measured error vector at each target $\left(\vec{e}_{\mathrm{T}}\right)$ with the vectors predicted by the two hypotheses. The residual errors were computed as

$$
\begin{gathered}
\tau_{T}=\left(\operatorname{angle}\left(\vec{e}_{T}\right)-\operatorname{angle}\left(\vec{t}_{T}\right)\right) / \delta_{T} \\
\rho_{T}=\left(\operatorname{angle}\left(\vec{r}_{T}\right)-\operatorname{angle}\left(\vec{e}_{T}\right)\right) / \delta_{T}
\end{gathered}
$$

for the translation and rotation hypothesis, respectively. Note that $\delta_{\mathrm{T}}$ denotes the maximal angular difference that the alternative predictions according to the two hypotheses would differ for each probe target. The residual errors were normalized by $\delta_{\mathrm{T}}$ to take the different size of the expected difference for the different probe targets into account. The residual error will be close to 0 if the data fit the tested hypothesis and close to 1 if the data fit the alternative hypothesis.

Testing the translation against the rotation hypothesis requires an effect of adaptation at the respective probe target in the respective subject. If endpoints do not significantly deviate from the target position, the error vector and the residual errors become random. This would likely affect especially probe targets with large distance from the adapted target. Therefore, we computed the average residual errors across subjects at each probe target position only for subjects in which reach directions in the control and test conditions were significantly different (ranksum test).

\section{Asymmetry of generalization}

We tested whether the generalization showed an asymmetry between probe targets which lie in the direction of the target jump (relative target position $>0$, per definition) compared to probe targets in the opposite direction $(<0)$. Note that this definition for the sign of the probe targets allows us the averaging of the data of the $\mathrm{CCW}$ and $\mathrm{CW}$ adaptation to extract effects which are specific for the direction of jump, and independent of the absolute direction in the workspace. We quantified the amount of transfer of adaptation to probe target positions in three different ways. First we analyzed the directional reach error (as used for the time course of adaptation above). Second and third the amount was defined by projecting the measured error 
vector onto the direction of the error vector as predicted by the translation hypothesis and the rotation hypothesis, respectively. This means, the relative adaptation for each probe target $T$ was for the translation hypothesis defined as:

$$
R A=\frac{\left\langle\vec{e}_{T} \cdot \vec{t}_{\mathrm{T}}\right\rangle}{\left|\vec{t}_{T}\right|}=\frac{\left|\vec{e}_{T}\right|}{\vec{t}_{T}} \cos \left(\operatorname{angle}\left(\vec{e}_{T}\right)-\operatorname{angle}\left(\vec{t}_{T}\right)\right)
$$

And for the rotation hypothesis defined as

$$
R A=\frac{\left\langle\vec{e}_{T} \cdot \vec{r}_{\mathrm{T}}\right\rangle}{\left|\vec{r}_{T}\right|}=\frac{\left|\vec{e}_{T}\right|}{\vec{r}_{T}} \cos \left(\operatorname{angle}\left(\vec{e}_{T}\right)-\operatorname{angle}\left(\vec{r}_{T}\right)\right),
$$

with <·> being the dot product. Note that the below results on the symmetry/asymmetry of generalization did not depend on this optimized quantification of the adaptation transfer. The results would hold equally true if symmetry/asymmetry were tested with the simple directional reach error, an observation which will be important for the comparison with the model data (see Results).

The resulting generalization function $(=R A(T))$ was fitted with a skewed t-distribution (Azzalini and Capitanio, 2003):

$$
\begin{aligned}
& t_{\text {skewed }}=\frac{2 a}{\sigma} \operatorname{tpdf}(z, d f) \operatorname{tcdf}(p, d f+1) \\
& z=\frac{x-\mu}{\sigma} \\
& p=\lambda z \sqrt{\frac{1+d f}{z^{2}+d f}} \\
& \operatorname{tpdf}(x, v)=\frac{\Gamma\left(\frac{v+1}{2}\right)}{\frac{v}{2}} \frac{1}{\sqrt{v \pi}} \frac{1}{\left(1+\frac{x^{2}}{v}\right)^{\frac{v+1}{2}}} \\
& \operatorname{tcdf}(x, v)=\int_{-\infty}^{x} \operatorname{tpdf}(x, v) \mathrm{dx}
\end{aligned}
$$

$\mu$ defines the center of the distribution (not the mean), $\sigma$ the width, and $\lambda$ the skewness. $\alpha$ defines a scaling factor and $d f$ the degrees of freedom. $\lambda$ was used to quantify the asymmetry of the generalization function. This function was fitted to the mean behavioral data across subjects on a least-mean-square basis. Individual data 
points were weighted with the inverse of their standard error (Matlab Curve fitting toolbox).

Only correct trials were included in the analysis. Yet, adaptation effects might occur in trials which were not finished correctly, but nevertheless provided some kind of feedback to the subjects. This could be trials with fixation breaks during the targethold time after the reach, or trials in which the target position was acquired, but not fast enough. We ran the same analyses including such trials. The results led to the same conclusions as when excluding these trials.

\section{Exclusion of subject}

Only subjects were analyzed who performed the task without difficulties. First, subjects with a success rate of $<40 \%$ in the initial test condition were excluded from the analysis (1 subject each in the $\mathrm{CW}$ and $\mathrm{CCW}$ experiment). This did not exclude several subjects whose scores were low due to eye fixation breaks, but whose performance otherwise was satisfactory. Second, subjects who reported that they had noticed the target jump were also excluded from the analysis (11 in CCW, 4 in $\mathrm{CW}$ ). We choose to exclude subjects who noticed the target jump as a conservative approach, even though they show similar results in several aspects (see Results). Third, subjects who did not show significant adaptation at the AD during the generalization period (directional reach error at $\mathrm{AD}$ not significant larger than zero, Wilcoxon signed rank test) were not included in the analysis (1 in CCW, 2 in $\mathrm{CW}$ ). The reduced dataset consisted of $16(\mathrm{CCW})$ plus $13(\mathrm{CW})$ subjects.

\section{Model simulations}

\section{Neural network model}

We used a three-layer recurrent neural network model to simulate our target-jump adaptation experiment. The idea was to test the changes of the neuronal representations in the network as a result of re-training the network from an initially congruent 1-to-1 mapping between sensory input and motor output to a locally perturbed input-output mapping. The model was nearly identical to a model, developed in a previous study, which had successfully performed flexible, contextspecific sensory-motor transformations (Brozovic et al., 2007). 
In brief, the model includes topographically organized input and output layer neurons which represent the one-dimensional workspace of the sensory input and the motor output, here the circular space of reach directions. Input layer neurons have Gaussian tuning profiles and tile the workspace evenly. Output layer neurons developed such tuning profiles after the network was trained from an initially random connectivity to a state in which it produces population tuning with a circular Gaussian profile (von Mises function) in response to local input (see below). The intermediate (hidden) layer with recurrent connections receives direct information from the input layer and is reciprocally connected with the output layer (Fig. 5A; Network II in (Brozovic et al., 2007)). All connection weights were updated according to a supervised backpropagation-through-time (BPTT) learning algorithm. The detailed implementation of the network and algorithm can be found in Brozovic et al. (Brozovic et al., 2007).

Only one modification to the original model was necessary to simulate the current experiment. The network was trained to only one mapping rule at a time (see training procedure below), whereas the original model learned four mapping rules in parallel. Importantly, no context input existed in the current version and the network had no explicit information about the exposure or non-exposure to the target jump. This network design mimics the consequences of the saccadic suppression in our experiment, namely, the fact that subjects did not explicitly know about the perturbation trials. The only source for driving learning is the target error at the end of the trial.

Additional minor modifications were made for 'aesthetic' reasons, namely to get smooth tuning functions and incremental adaptation with a learning rate similar to the experiments. The number of input/output neurons was increased to 180 and the number of hidden layer unit was increased to 50. This allowed simulations with a wide range of tuning widths (see below), while still covering the full workspace densely. We used a population vector algorithm to quantify the model's motor output, i.e. the 'reach' direction was decoded via the sum of vectors defined by the preferred direction of each output neuron weighted with its activation level (Georgopoulos et al., 1986). 


\section{Simulation procedure}

We trained the model in the following steps to simulate the target jump adaptation paradigm: (1) Standard mapping: Starting from a random-weights initialization, the network was trained to perform a 1-to-1 mapping of the visual input to a congruent motor output across the full workspace. Standard mapping mimics standard trials of our experiment. A circular Gaussian population response across output layer neurons with a predefined width and centered on the desired reach direction served as teaching signal. Training was continued until the mean square error between the desired and actual activity profile across output layer neurons dropped below 0.001. (2) Target jump: Starting from the model which performed the standard mapping, the model then was trained to perform a rotated mapping $\left(15^{\circ}\right)$ for a single input direction (adapted direction, $\mathrm{AD}$ ). The training algorithm was identical to the standard mapping. These trials simulated the exposure phase with target jumps in our experiment. (3) Generalization: After the model learned the rotated mapping in one direction, the synaptic weights were kept fixed to mimic probe trials without feedback, i.e. without an error signal. We then probed the model across the workspace to test how adaptation at one location generalizes to other untrained targets, i.e., to assess postadaptation generalization.

Note, the workspace of the model is inherently 1-dimensional. For comparison with human generalization data, the model reach error was defined as the angular deviation of the model reach direction in the generalization (phase 3) from the model reach direction after learning the initial standard mapping (end of phase 1), normalized to the size of the target jump. The quantitative differences between translational and rotational generalization can not be addressed with this 1-D model, but the qualitative testing for symmetry/asymmetry in generalization is independent of this.

The tuning width within input and within output neurons was constant (see Brozovic et al. 2007 for definition of tuning width). The motor tuning width was always equal or larger than the visual tuning width. Both tuning widths were varied in the range between $\sigma=15^{\circ}$ and $75^{\circ}$ in steps of $10^{\circ}$ to find the combination which best matched the human data (see Results). 
We ran all simulations with 30 different sets of random initialization. The 30 seeds in the analysis of the model data were treated equivalently to the different subjects of the experimental data.

\section{Model-neuron tuning analyses}

Directional selectivity and preferred direction (PD) of output and hidden layer neurons was quantified with a directional tuning vector (DTV), which is defined as the vector average across all spatial input directions $\vec{u}_{i}$ (unit vectors) weighted with the response $r_{i j}$ of neuron $j$ to input at direction $i$, normalized to the average response of neuron $j$ :

$$
D T V_{j}=\frac{\sum_{i=1}^{n} r_{i j} \vec{u}_{i}}{\frac{1}{n} \sum_{i=1}^{n} r_{i j}}
$$

For defining peak activity and tuning width the tuning curves of the neurons were interpolated by a factor of 5 ('ideal' low-passing via fast Fourier-transform; MATLAB function interpft) leading to a nominal $1^{\circ}$ resolution. Peak activity was defined as the activity at the PD of the neuron. Tuning width was defined as full width at half maximum (FWHM). Changes in PD, tuning width and activity are analyzed as difference:

$\Delta$ Parameter $=$ Parameter $_{\text {Generalization }}-$ Parameter $_{\text {Standard }}$ 


\section{Results:}

Subjects followed the instructions and conducted the saccade and reach simultaneously. In the generalization task the reach reaction times were $226 \pm 29 \mathrm{~ms}$ (mean \pm standard deviation) and the saccadic reaction times $215 \pm 24 \mathrm{~ms}$. In the control task reach reaction times were $239 \pm 34 \mathrm{~ms}$ and the saccadic reaction times $225 \pm 32 \mathrm{~ms}$.

\section{Target jump adaptation}
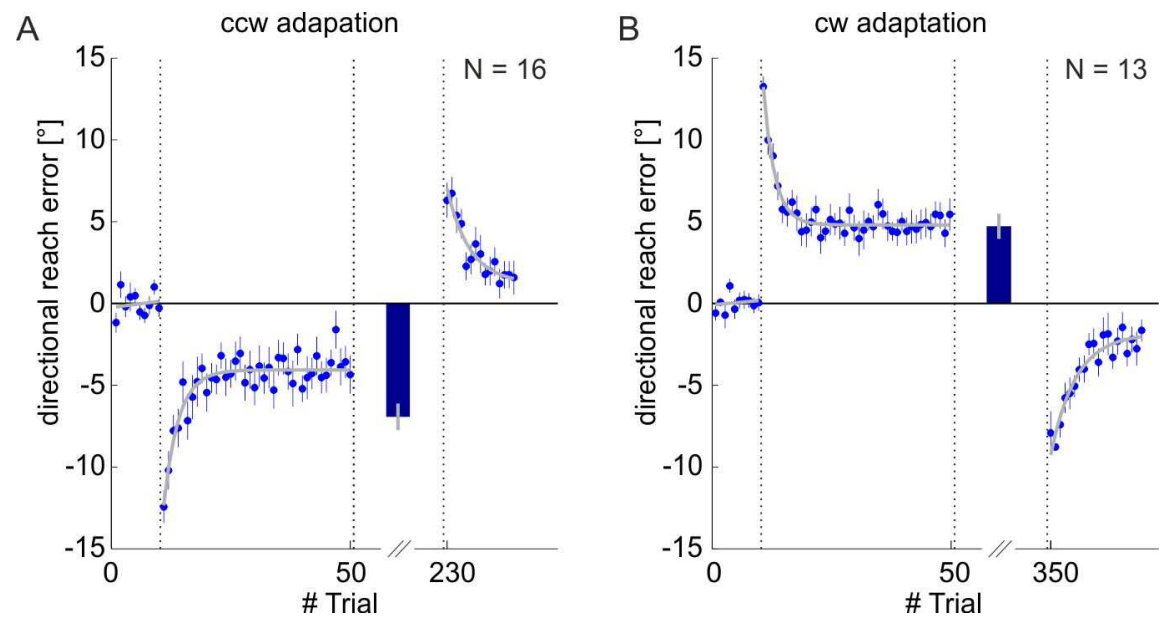

Figure 2 Learning curves. The average directional reach error (mean \pm s.e.m., baseline corrected) across $\mathrm{N}=16$ and $\mathrm{N}=12$ subjects is shown for $\mathrm{CCW}$ (left) and $\mathrm{CW}$ (right) adaptation and fitted with an exponential (gray curves) in exposure and post-exposure periods. The bar in the generalization period reflects the mean directional reach error $( \pm$ s.e.m.) for the target jump trials presented during this period.

Our target jump paradigm reliably induced reach adaptation. This is shown by an incremental reduction of the reach error during exposure and the negative aftereffect in the post-exposure period (Fig. 2 and Table 1). The learning rates in the exposure were $-0.33^{\circ}$ and $-0.43^{\circ}$ per trial and the initial directional reach errors in the postexposure were $7.0^{\circ}$ and $9.8^{\circ}$ for the $\mathrm{CCW}$ and $\mathrm{CW}$ experiments. All values are significantly different from zero (95\% confidence limits, see Table 1). Adaptation was incomplete and the size of the initial reach error during post-exposure approximately matched the difference between the initial reach error during exposure and the 
asymptotic remaining reach error ('offset') at the end of the exposure period. This difference is also the level of adaptation that was sustained during the generalization period.

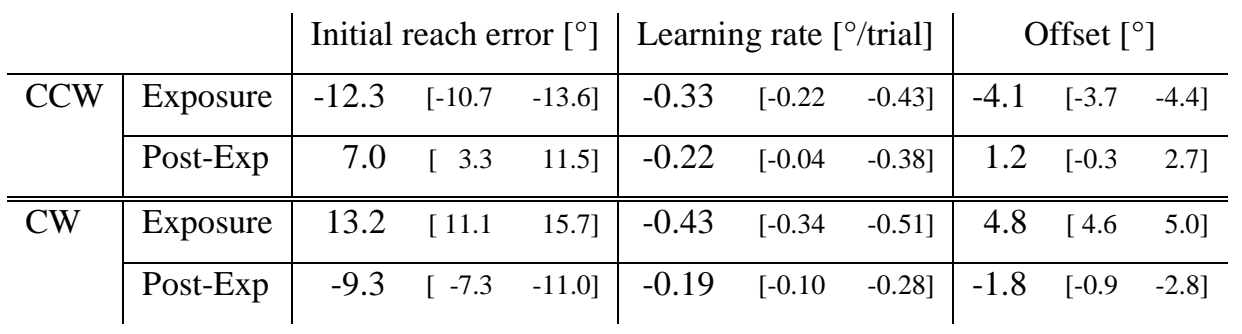

Table 1: Initial directional reach error, learning rate and asymptotic reach error (offset) as derived from an exponential fit for the exposure and post-exposure period in the CCW and CW experiment. Values in brackets give the $95 \%$ confidence intervals.

Since the results from the $\mathrm{CCW}$ and $\mathrm{CW}$ generalization were not qualitatively different they will be presented jointly in the following.

As a control experiment, we tried to induce reach adaptation with a target jump paradigm, but without the accompanying saccade. In this case subjects $(\mathrm{N}=4)$ did not show incremental improvement or a negative aftereffect. Reach endpoints were only significantly different from the displaced target position in the first trial after beginning of the exposure period or the first trial after beginning of the post-exposure, but then performance immediately returned to baseline in the second trials (data not shown). Since these results are consistent with previous studies (Diedrichsen et al., 2005), we did not further investigate this condition. This control confirms the existing view that a consistent and noticeable target jump due to its predictability leads to a strategic updating of the intended reach goal, rather than an implicit adaptation, in otherwise identical task conditions.

The main results of human psychophysics contain only data from subjects who reported not to have seen the target jump (see Methods). As a second control, we also analyzed the subpopulation of subjects who reported to occasionally have seen the target jump. The fraction of subjects who did show significant adaptation within the group with only partial suppression of target jump visibility (13 out of 15) was not 
smaller than the fraction in the group of subjects who reported fully successful suppression (29 out of 32, p $=0.92$, Pearson's $X^{2}$ - test with Yates' correction for continuity). Since we did not probe and quantify the subjects' abilities of detecting jumps, we were not able to correlate the degree of subjective suppression of the jump with the degree of adaptation. Subjects never reported to have seen the jump consistently in every exposure trial, which is probably the reason why the general adaptation effects were not abolished (see generalization results below for differences between both subpopulations).

In summary, the incremental improvement in reach performance and significant negative aftereffects confirm that implicit adaptation can be induced by noticeable target errors alone, without peri-movement prediction errors or cross-modal mismatch, as long as the target jump as the source of the target error is rendered invisible and the target believed to be stationary by the subjects.

\section{Generalization of endpoint position}

After establishing the adaptation to target jump, our second main goal was to characterize this form of adaptation in terms of its generalization. Our paradigm is different from previously tested paradigms and we tested generalization of reach endpoints (2D), rather than initial reach direction (1D). Therefore, we needed to establish in which way the learning effect transfers to other directions before we could precisely quantify the amount of generalization. In the individual example subject (Fig. 3A), the average reach endpoints largely coincide with the predicted direction of reach errors according to the translation hypothesis ( $\vec{t}$, red arrows) but not according to the rotation hypothesis ( $\vec{r}$, blue arrows) (Fig. 3B, see Methods). In the example data and across the population of subjects (Fig. 3C), the generalization pattern fits the translation hypothesis and rejects the rotation hypothesis. The average residual error for the translation hypothesis across all subject and all targets is -0.031 , which is not significantly different from $0(\mathrm{p}=0.77$, $\mathrm{t}$-test $)$. The average residual error for the rotation hypothesis is 1.075 , which is significantly larger than $0\left(\mathrm{p}<10^{-7}\right.$, t-test). Note that values around zero for one hypothesis automatically lead to values around 1 for the other hypothesis. But the fact that the average residual error for the rotation 
hypothesis is significantly larger than 0 , contains the important information that the non-significant result of the translation hypothesis is not due to a lack of statistical power.

The observed translational generalization of reach endpoints suggests that target jump adaptation happens at the task level of motor goal representations in a visuospatial reference frame, rather than at an implementation level with independent encoding of reach direction and amplitude.
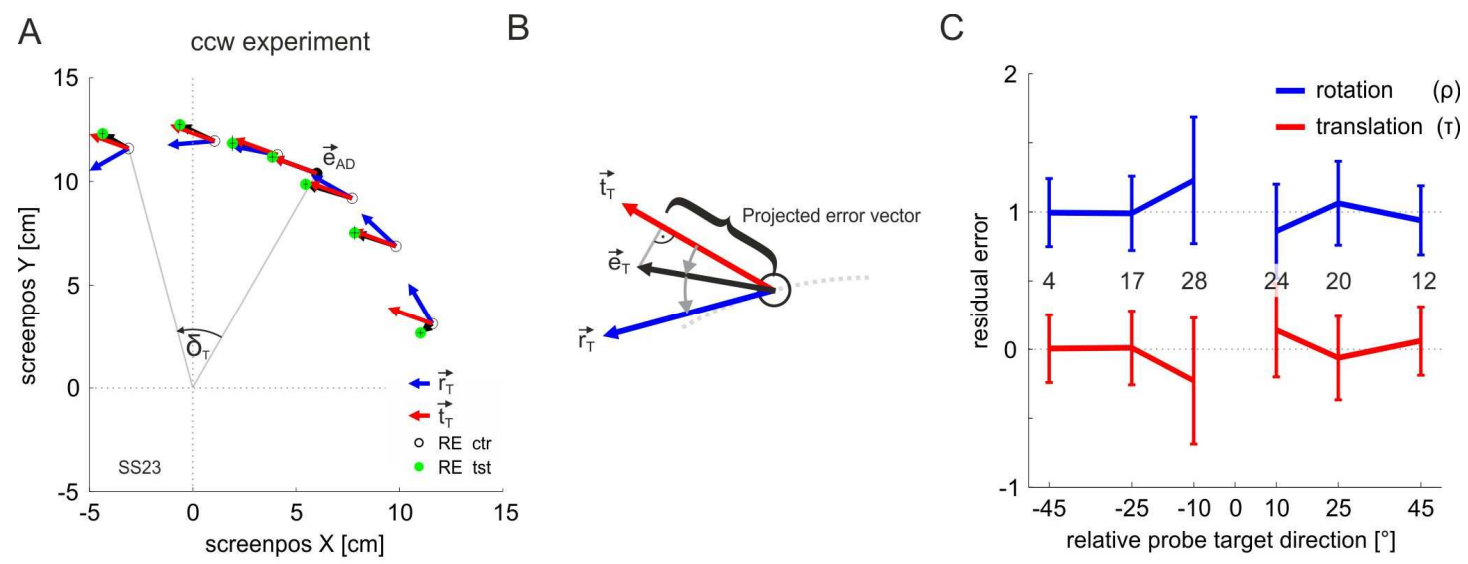

Figure 3 Translational versus rotational generalization. A: Average reach endpoints (RE) in the control condition (ctr, open circles) and the test condition (tst, green circles) for one example subject in CCW generalization. Red and blue arrows depict the predictions for the translation $\left(\vec{t}_{T}\right)$ and rotation $\left(\vec{r}_{T}\right)$ hypothesis at each probe target position. Black arrows depict the subject's actual reach error $\left(\vec{e}_{T}\right)$. B: Enlarged illustration of the error vector with the predictions for translation and rotation hypothesis at one probe target. The angular difference between $\vec{e}_{T}$ and $\vec{t}_{T}$ divided by the maximum to-be-expected angular difference $\delta_{T}$ between both hypotheses defines the residual error $\tau_{T}$. The angular difference between $\vec{r}_{T}$ and $\vec{e}_{T}$ divided by $\delta_{T}$ defines the residual error $\rho_{T}$. C: Residual error for rotation $\left(\rho_{T}\right.$, blue $)$ and translation $\left(\tau_{T}\right.$, red) hypothesis at different probe targets for pooled data from the $\mathrm{CW}$ and $\mathrm{CCW}$ experiment. Probe target positions (x-axis) are labeled relative to the $\mathrm{AD}$ at $60^{\circ}$. At each probe target only those subjects are included with show a significant difference in direction between the test and the control condition (see numbers within figure). 


\section{Asymmetry of generalization}

After establishing the direction along which adaptation transfers, we quantified the amount of transfer as a function of distance from the AD. Average reach trajectories in the adapted condition are shifted into the direction of the target jump (Fig. 4A), especially for probe targets neighboring the $\mathrm{AD}$, and less so for probe targets further away from the AD. The transfer of adaptation in this example subject was stronger for probe targets on the side of the jump target (clockwise to $\mathrm{AD}$ ), compared to probe targets on the opposite side. In the previous section we show that adaptation to the target jump paradigm follows a translational hypothesis. Therefore, for the population analysis, we quantify the relative amount of adaptation in the following as projection on the predicted translation vector (see methods). According to the example subject, the relative adaptation on average across subjects (Fig. 4B) decreased with increasing distance from the $\mathrm{AD}$, which results in significant differences in the size of the adaptation effect for the different target positions $\left(\mathrm{p}<10^{-9}\right.$, repeated measurement ANOVA).
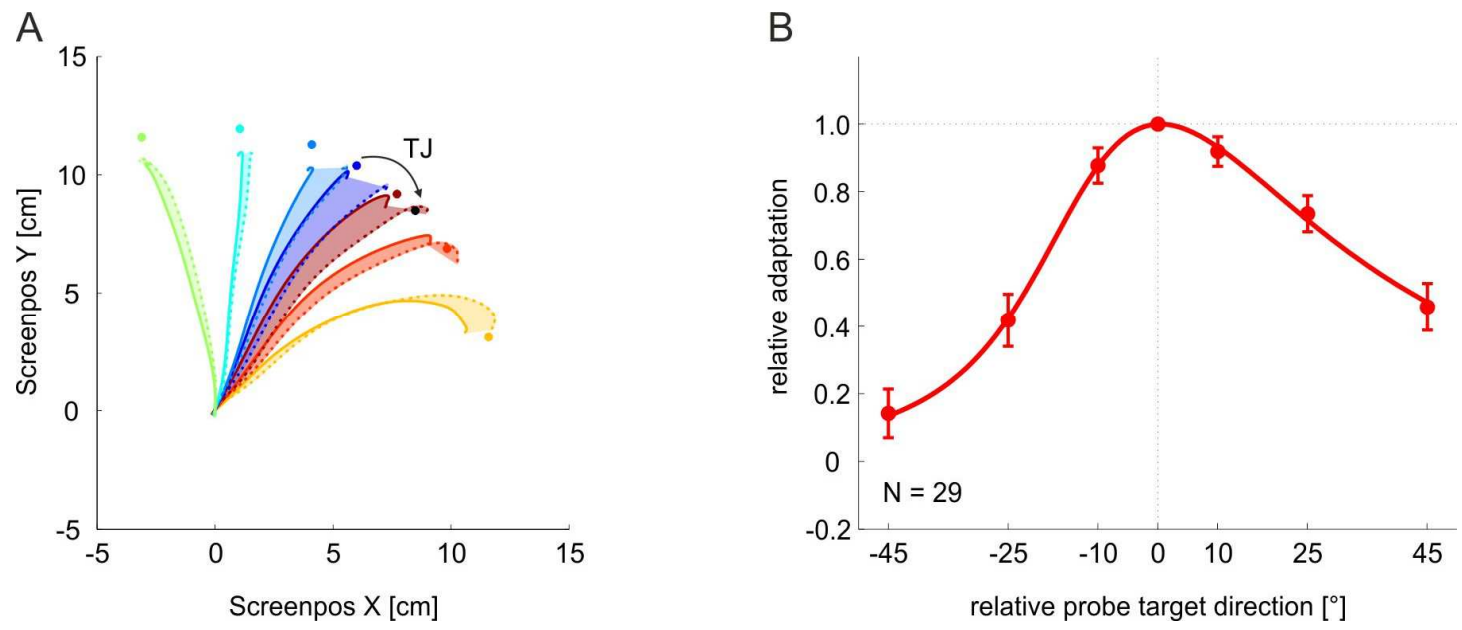

Figure 4 Generalization pattern of target jump adaptation. A: Average reach trajectories for the different probe target positions during the control (solid) and test (dashed) condition for an example subject in the $\mathrm{CW}$ generalization. Trajectories are corrected for an initial offset during central fixation. B: Average relative adaptation (mean \pm s.e.m.) for pooled data from the $\mathrm{CW}$ and $\mathrm{CCW}$ experiment, fitted with a skewed t-distribution. Probe target positions are defined relative to the $\mathrm{AD}$ at $60^{\circ}$. Positive deviations are defined as probe targets into the direction of the target jump, negative deviations are probe targets in the direction opposite to the jump. 
Besides the drop-off with distance, the generalization showed an asymmetry. Probe targets in the direction of the jump (relative target direction $>0$ ) showed larger relative adaptation than probe targets in the opposite direction of the jump. We quantified this asymmetry by fitting a skew t-distribution to the average generalization data. The positive $\lambda$ of 1.91 (95\% confidence interval [0.65 3.17]) indicated a rightwards skewness, i.e., larger adaptation effect in the direction of the jump. We found a trend towards the same generalization pattern, but the skewness was smaller and not significantly different from zero, when subjects reported that they had noticed the jump occasionally $(\lambda=1.02$, CI [-1.67 3.7], $\mathrm{N}=13)$.

The results show that in our target jump paradigm the adaptation generalizes asymmetrically and more strongly towards the side of the perturbation. This is different to visuomotor rotation experiments, which show symmetric generalization. While the results suggest that a skewed generalization could depend on a fully successful suppression of jump visibility, we can not rule out that a lack of statistical power $(\mathrm{N}=13$ compared to $\mathrm{N}=29)$ is partly responsible for the non-significant skewness in the subgroup of subjects who occasionally noticed the target jump.

\section{Neural network simulations}

We used a recurrent multi-layer neuronal network model (Fig. 5A) to simulate the adaptation learning in our task and investigated the associated changes in the underlying neuronal network structure (see Methods). We first established that the model's motor output in generalization trials was consistent with human behavior. The transfer of adaptation to non-adapted probe targets significantly decreased with increasing distance from the $\mathrm{AD}\left(\mathrm{p}<10^{-10}\right.$, repeated measurement ANOVA). Importantly, the model produced significant asymmetric generalization $(\lambda=1.32, \mathrm{CI}$ [1.06 1.58]) within the range of the confidence limits of the experimental data (Fig. $5 \mathrm{~B})$. Note that the model was not trained to produce asymmetry. The motor layer output during initial training and exposure was trained to produce symmetric population responses, which for the $\mathrm{AD}$ during adaptation where shifted relative to the control task. 

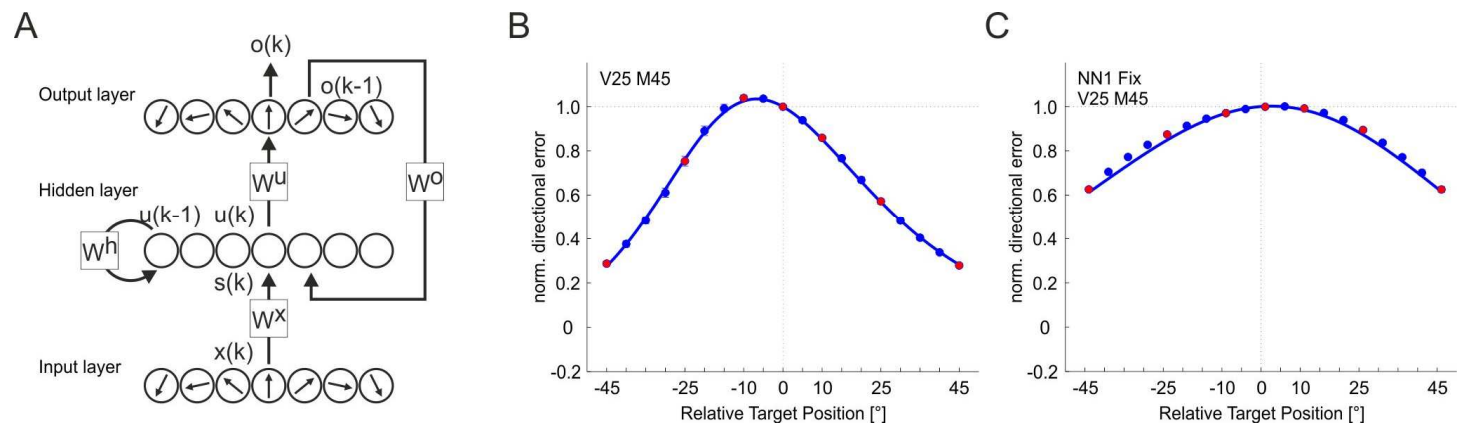

Figure 5 Simulation of target jump adaptation with a recurrent neural network model. A: Network architecture. The input (sensory) layer encodes the position of the visual stimulus. The hidden layer receives direct input from the sensory layer and input via feedback connection from the output layer. Additionally, the hidden layer units are recurrently connected. The total input into the hidden layer is defined as $s(k)=W^{x} x(k)+W^{h} u(k-1)+W^{o} o(k-1)$, with W defining weight matrices and $\mathrm{k}$ defining time steps. The output of the hidden layer is defined as $u(k)=\varphi(s(k))$, with $\varphi$ being a sigmoid transfer function. The output (motor) layer receives the hidden layer activation as input $o(k)=\varphi\left(W^{u} u(k)\right)$. Reach direction is decoded with a population vector based on the output layer activities. For details see (Brozovic et al., 2007) and Methods. B: Asymmetric generalization curve of the model for an example simulation with tuning width of $25^{\circ}$ for visual units (V25) and $45^{\circ}$ for motor units (M45). Data points show the average normalized directional error (mean \pm s.e.m.) over 30 model simulations with independent random initialization. The red colored data points highlight probe target positions which were also used in the behavioral experiment. Other conventions are the same as in figure 4B. C: Symmetric generalization curve of a variation of the model in which fixed tuning properties in the hidden layer were enforced (see text). Conventions are the same as for B.

We tested the asymmetry in generalization with various combinations of tuning width for the input (visual tuning V) and output layer neurons (motor tuning $\mathrm{M}$ ), which are the free parameters of the model $\left(\sigma=25^{\circ}\right.$ to $75^{\circ}$ in steps of $10^{\circ}$ for input and output units, with $\sigma_{\mathrm{V}} \leq \sigma_{\mathrm{M}}$ ). The model produced asymmetric generalization which overlapped in strength with the confidence limits of the human data for a wide range of the tested tuning widths (all but V25M35, V25M65, V35M35, V35M75, V45V45, V55M75; data not shown). Note that the model did not match the shift in center $(\mu)$ of the experimental generalization curve $(\mu=-15.6[-20.2-11.0])$ for a wide range of tuning widths. We quantified the tuning parameters of the model which best fit the shape of the human data independent of the center shift on the basis of the mean square error (MSE). The best fit between model and human data was achieved with a 
tuning width of $25^{\circ}$ for the input neurons and of $45^{\circ}$ for the output neurons. The discrepancy of $\mu$ between human and model data was $11^{\circ}$ for this parameter setting.

Previous computational studies simulated adaptation with symmetric generalization and suggested that the tuning in visuomotor areas should not change during visuomotor rotation (Tanaka et al., 2009). We tested whether the asymmetric generalization in our model depends on tuning property changes in the hidden (=visuomotor) layer, or if changes in the projections from the hidden to motor layer are sufficient to reproduce the behavioral data. We ran a model simulation in which the weights from the input to the hidden layer, as well as the weights of the recurrent connections within the hidden layer were fixed after learning the standard mapping. Additionally, the network architecture was changed such that the feedback connections from output to hidden layer were removed during all phases. All three measures in combination were necessary to create a situation in which the tuning in the hidden layer could be kept unchanged. With the fixed tuning properties of the hidden layer, the model was still able to learn the target jump, but the generalization no longer showed a positive skewness $(\lambda=-1.14$, CI [-2.41 0.13]) (Fig. 5C).

These simulations suggest that without changes of the tuning properties in the hidden layer of the model (the putative visuomotor areas of the brain) asymmetric generalization can not be explained. The fact that in the model changes of motor goals lead to a skewed generalization, suggests that the skewed generalization in the human data is also based on changes of motor goals in response to adaptation to target jump.

We analyzed the tuning changes of units in the hidden and the output layer of the network to learn how adaptation to the target jump was achieved. Figure 6A shows for all neurons of the network across 30 different random initializations how preferred direction (PD), tuning width, and peak activity changed after adaptation to target jump (see Methods). Several observations can be made. First, changes in the output and hidden layer are qualitatively very similar, where tuning changes in the hidden layer show more variability than changes in the output layer. Second, model units with a $\mathrm{PD}$ identical to the $\mathrm{AD}\left(\mathrm{PD}=0^{\circ}\right)$ and with a $\mathrm{PD}$ opposite to the $\mathrm{AD}\left(\mathrm{PD}=180^{\circ}\right)$ are least affected by tuning changes. Units with PDs at $-45^{\circ} \pm 5^{\circ}$ (opposite to the side of the 
jump) and $+65^{\circ} \pm 5^{\circ}$ (same side as jump) relative to $\mathrm{AD}$ showed the maximal changes in their PDs. These are units for which the flanks of the tuning curves overlap well with the AD. Third, additional to the asymmetry in the PDs at which there is a maximum effect on tuning, we also quantified whether there is an asymmetry in the amount of change to the tuning for the neurons with a PD at the same or opposite side of the jump. The maximal change of PD and tuning width was stronger on the side of the target jump. In the output layer PD decreased by $4.40^{\circ}$ at the side of the jump compared to a decrease of $4.27^{\circ}$ at the opposite side $(\mathrm{p}=0.045$, rank sum test). The tuning width increased by $17.2^{\circ}$ on the side of the target jump compared to a decrease of $18.0^{\circ}$ at the opposite side $(\mathrm{p}=0.0026)$. The change in peak activity was stronger on the side opposite to the target jump. In the output layer the peak activity increased by 0.004 on the side of the target jump compared to a decrease of 0.009 at the opposite side $\left(\mathrm{p}<10^{-7}\right)$. For the hidden layer the asymmetry in the strengths of the effects (independent of sign) were not significant due to the large variability in the hidden layer tuning. Note, the observed differences between the directions in and opposite to the jump direction increase in absolute size when teaching the network to larger jumps of $45^{\circ}$ and $90^{\circ}$, compared to the $15^{\circ}$ jump shown here (data not shown).

The combination of changes in PD and tuning width can be explained by specific changes to the shape of the tuning curves of the affected units. Figure 6B shows average tuning curves of maximally affected hidden and output layer units with PDs at the $\mathrm{AD}$ and at $-45^{\circ} \pm 5^{\circ}$ and $+65^{\circ} \pm 5^{\circ}$ from the $\mathrm{AD}$ before and after the adaptation. The tuning changes mainly the activity at only one flank of the tuning curve. This results in a combination of changes in PD and tuning width, but less so in changes of peak height.. Units with a PD opposite to the target jump showed a decrease of their activity at the flank overlapping the AD (falling flank), whereas units with a PD in the direction of the target jump showed an increase at the flank overlapping the AD (rising flank). This opposing change in the tuning width caused the PD of both types of units to move away from the direction of the target jump. Units with their PD close to $180^{\circ}$ apart from the AD show comparably small effects and shift their whole tuning curve instead of changing the activity at one flank only (Fig. 6C). 
A
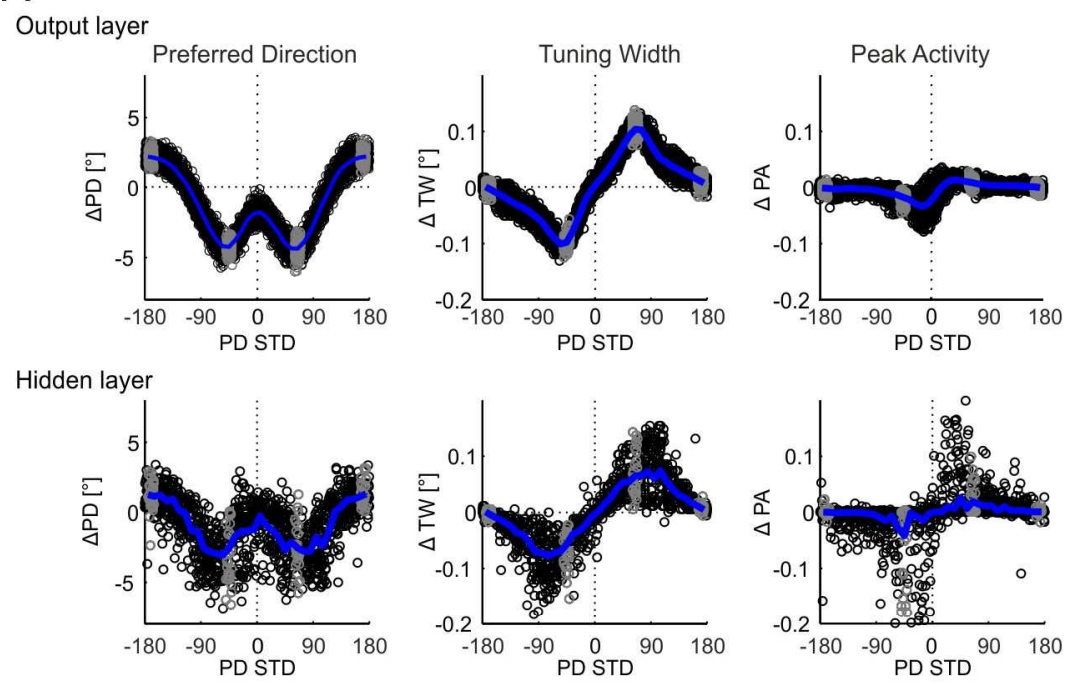

B

C
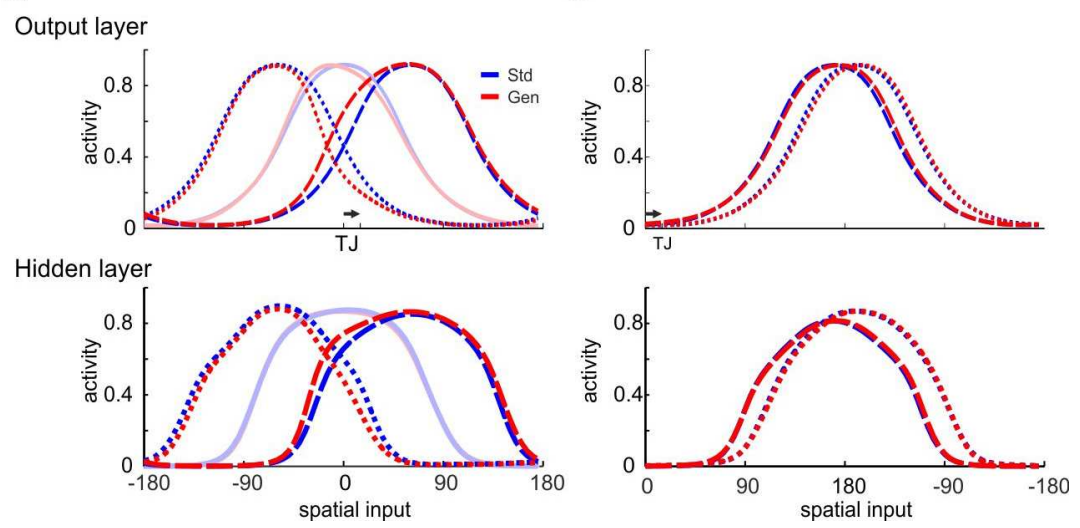

Figure 6 Tuning changes (after adaptation-before adaptation) in hidden and output layer of the model due to adaptation. A: Adaptation-induced change of PD (left), tuning width (middle), and peak activity (right) in each of the 180 units of the output layer (top row) and 50 units of the hidden layer (bottom row), sampled across 30 independent random network initializations. The units are sorted according to the PD before adaptation (x-axis). The blue curve plots the mean \pm s.e.m. for $10^{\circ}$ bins. Units in gray depict those whose average is plotted in B and C. B: Average tuning curves for units in hidden (bottom) and output (top) layer, which have a PD of $-45 \pm 5^{\circ}$ (dotted), $0 \pm 5^{\circ}$ (solid) and $+65 \pm 5^{\circ}$ (dashed). PDs were chosen to be at the $10^{\circ}$ bins where adaptation-induced PD changes in the output layer were strongest (see Figure 6A left). Tuning curves before adaptation (Std) are shown in blue, tuning curves after adaptation (Gen) are shown in red. C: Same as B but for units which have a PD of $-170^{\circ} \pm 5^{\circ}$ (dotted) and $+170^{\circ} \pm 5^{\circ}$ (dashed). Note the shifted $\mathrm{x}$-axis. 


\section{Discussion}

Adaptation behavior can reveal important insights into the nervous system function, where different types of perturbation seem to engage adaptation at different levels of the sensorimotor system (Shadmehr and Wise, 2005). We developed a perturbed reaching paradigm in which we displaced the reach target during saccadic suppression early in the reaching movements, and which induced implicit adaptation by target error alone. We found generalization patterns different to those reported in kinematic adaptation paradigms with visual feedback perturbation. With a neural network model we were able to simulate the behavioral adaptation and make predictions about neural tuning changes in visuomotor areas putatively underlying this adaptation.

\section{Adaptation to target jump}

Perturbing feedback during reaching movements often leads to sensorimotor adaptation (Shadmehr and Mussa-Ivaldi, 1994; Kitazawa et al., 1995; Martin et al., 1996; Vetter et al., 1999; Thoroughman and Shadmehr, 1999; Krakauer et al., 2000; Paz et al., 2003; Redding and Wallace, 2006). In most experiments adaptation could be driven by a combination of prediction and target error. It has been shown, though, that prediction error alone can drive adaptation (Mazzoni and Krakauer, 2006; Tseng et al., 2007). Here we show adaptation to target error alone, which so far was believed to trigger adaptation if at all then only under highly restricted conditions, since previous efforts to generate adaptation in response to target jump had faced serious difficulties. When a target jump is noticeable to subjects as a sudden displacement, no negative aftereffects are observed during post-exposure. This has been shown previously (Diedrichsen et al., 2005; Cameron et al., 2010) and confirmed here. This suggested that noticeable target jumps engage explicit changes in strategy rather than implicit adaptation mechanisms. Tasks in which the target jump was perceptually suppressed by a simultaneous saccade to the reach target itself lead to saccadic adaptation (Bekkering et al., 1995). Such task design makes it difficult to distinguish reach adaptation from changes in motor goals secondary to saccade adaptation, since it has been shown that the error signal of the saccade system (also independently of adaptation) can be used by the reach system to generate online corrections to reaching movements (Pelisson et al., 1986; Prablanc and Martin, 1992; Bekkering et al., 1995; De Graaf et al., 1995). Other studies attempted to address this confound by 
introducing unperturbed saccade trials to prevent saccadic adaptation. In this case, shifts of reach endpoints could only be gradually induced with successive and unnoticeably small target displacements, while the view of the hand was blocked during the movement (Magescas and Prablanc, 2006; Magescas et al., 2009; Cameron et al., 2010), Thus, it has been the consensus of the field that reach adaptation is possible in target jump only if the hand-to-target error is small enough to be interpreted as "natural" planning error (Magescas et al., 2009). Very recently, target jump adaptation could also be shown for noticeably large jumps without saccadic coadaptation, but generalization was not analyzed (Cameron et al., 2011).

In summary, our study shows that target jump adaptation is also possible for large and clearly noticeable target errors during unperturbed and unblocked direct vision of one's own hand. When saccadic suppression lets subjects perceive the peripheral target as stable despite the target jump, then their sensorimotor system seems to attribute the resulting target error to errors in the agents sensorimotor processing, rather than an update of the movement target in the distal world, and thereby allow adaptation.

\section{Adapting visuospatial motor goals}

Different adaptation paradigms should affect different parts of the sensorimotor system (Shadmehr and Wise, 2005). Visuomotor rotation tasks, as used, for example, in the experiments of Krakauer et al. (Krakauer et al., 2000), alter the mapping between where the actual hand moves and where the representing cursor moves. Adaptation in these experiments affected direction and extend of the reach separately (when perturbed individually), suggesting changes to reach-related neuronal representations at the level of movement implementation. The translational generalization pattern in our data suggests that target jump adaptation affects a part of the sensorimotor system in which motor goals are represented in visuospatial coordinates at the task level (see also discussion of model data in next section). Similar to our results, an experiment in which only the visual feedback about the hand endpoint position was perturbed showed translational generalization (Ghahramani et al., 1996). Yet, such visual feedback perturbation experiments are thought to affect the alignment of the visually and proprioceptively sensed locations of the hand (Ghahramani et al., 1996; Shadmehr and Wise, 2005). This might be a plausible explanation for cursor tasks which are characterized by an a priori artificial 
alignment of visual and proprioceptive inputs, and in which visuomotor rotation leads to a large misalignment in the localization of the exposed compared to the unexposed hand, but less to a mislocalization of the visual target (Simani et al., 2007). In contrast, it is not plausible that in our target jump a visuo-proprioceptive missalignment is induced, since the experimental setup leaves no doubt about hand position in either sensory modality.

\section{Changes in visuomotor tuning during target jump adaptation - a prediction}

The generalization pattern of adaptation can help to identify different forms of adaptation and reveal constraints for possible underlying neural mechanisms (Imamizu et al., 1995; Pine et al., 1996; Krakauer et al., 2000; Donchin et al., 2003; Shadmehr and Wise, 2005; Thoroughman and Taylor, 2005; Mattar and Ostry, 2007; Tanaka et al., 2009; Magescas et al., 2009; Mattar and Ostry, 2010). Generalization in kinematic visuomotor reach adaptation has mostly been reported to be symmetric (Pine et al., 1996; Krakauer et al., 2000; Tanaka et al., 2009). In two kinematic adaptation studies where asymmetry might have been observed it was neither quantified nor discussed (Ghahramani et al., 1996). Gonzalez Castro and colleagues (Gonzalez Castro et al., 2011) measured and quantified generalization in a force-field adaptation paradigm with blockwise alternating adaptation to $\mathrm{CW}$ and $\mathrm{CCW}$ force fields. They showed 'asymmetry' in the sense that a symmetric generalization pattern was shifted relatively to a fixed reference point, but there was no skewness in generalization, like in our data.

This means, the skewed generalization corroborates the idea that we observed a type of adaptation different to the phenomena previously reported, for which we wanted to understand how it could be achieved. We were able to simulate our human behavioral data using a recurrent three layer neural network. The model was originally designed to flexibly remap visual targets onto motor goals according to an explicit context input (Brozovic et al., 2007). The resulting tuning of the hidden layer model units closely matched neural tuning in parietal and premotor cortices of monkeys (Gail et al., 2009). We predict that, in an analog fashion, the observed tuning changes in our simulation of the target jump task could happen in visuospatial motor goal areas of the brain. Obviously, such model can only be a cartoon of the real neural system. But the observed skewed generalization in the target jump task gives our model some 
credibility, since it preexisted and was not modified in any way to account for such asymmetry. Our model robustly produced skewed generalization using a wide range of different parameter sets, but only when tuning changes in the hidden layer were allowed. When only changes of the mapping from the hidden layer to the output were allowed, the model produces symmetric generalization. Symmetric generalization was also shown in a previous computational study and has been associated with adaptation of visual-to-motor transformations (Tanaka et al., 2009) or visuo-proprioceptive realignment (Ghahramani et al., 1996), rather than with adaptation of visuospatial motor goals. In this sense the findings of the previous computational studies and our study are complementary. Our model tuning changes are also different from what has been observed in monkey primary motor cortex during adaptation to visumotor rotation, where only neurons with PDs close to the adapted movement increase their activity at their PD (Paz et al., 2003).

The results of our model are suggestive. The model mostly modified the relative representations between sensory input and hidden layer, while the changes between hidden and output layer where consistent. Effectively, this would correspond to a change in the transformation from sensory input to visuospatial motor goal representations in task space. Based on our simulations, we speculate that neurons in sensorimotor areas, e.g. the parietal reach region (PRR), change their tuning properties during adaptation to target jump in the specific manner described in our model.

\section{Conclusions}

Our novel target jump paradigm induced reach adaptation effects in response to target errors only. The observed generalization patterns suggest adaptation at the level of motor goal representations in a visual task space. Adaptation effects generalized asymmetrically and stronger in the direction of the target jump. Simulation of this asymmetry in a recurrent neuronal network model is contingent on tuning property changes in the hidden layer, suggesting adaptation based on neural tuning changes at early levels of visuospatial reach goal representations. 


\section{References}

Azzalini A, Capitanio A (2003) Distributions generated by perturbation of symmetry with emphasis on a multivariate skew t-distribution. Journal of the Royal Statistical Society: Series B (Statistical Methodology) 65: 367-389.

Bedford FL (1989) Constraints on learning new mappings between perceptual dimensions. Journal of Experimental Psychology: Human Perception and Performance 15: 232-248.

Bekkering H, Abrams RA, Pratt J (1995) Transfer of saccadic adaptation to the manual motor system. Human Movement Science 14: 155-164.

Bock O (1992) Adaptation of aimed arm movements to sensorimotor discordance: evidence for direction-independent gain control. Behavioural Brain Research 51: 4150 .

Brozovic M, Gail A, Andersen RA (2007) Gain Mechanisms for Contextually Guided Visuomotor Transformations. J Neurosci 27: 10588-10596.

Cameron BD, Franks IM, Inglis JT, Chua R (2010) Reach adaptation to explicit vs. implicit target error. Exp Brain Res 203: 367-380.

Cameron BD, Franks IM, Timothy IJ, Chua R (2011) Reach adaptation to online target error. Exp Brain Res 209: 171-180.

De Graaf JB, Pelisson D, Prablanc C, Goffart L (1995) Modifications in end positions of arm movements following short-term saccadic adaptation. Neuroreport 6: 17331736.

Diedrichsen J, Hashambhoy Y, Rane T, Shadmehr R (2005) Neural Correlates of Reach Errors. J Neurosci 25: 9919-9931.

Donchin O, Francis JT, Shadmehr R (2003) Quantifying generalization from trial-bytrial behavior of adaptive systems that learn with basis functions: Theory and experiments in human motor control. J Neurosci 23: 9032-9045.

Gail A, Klaes C, Westendorff S (2009) Implementation of Spatial Transformation Rules for Goal-Directed Reaching via Gain Modulation in Monkey Parietal and Premotor Cortex. J Neurosci 29: 9490-9499.

Georgopoulos AP, Schwartz AB, Kettner RE (1986) Neuronal population coding of movement direction. Science 233: 1416-1419.

Ghahramani Z, Wolpert DM, Jordan MI (1996) Generalization to Local Remappings of the Visuomotor Coordinate Transformation. J Neurosci 16: 7085-7096.

Gonzalez Castro LN, Monsen CB, Smith MA (2011) The binding of learning to action in motor adaptation. PLoS Comput Biol 7: e1002052. 
Imamizu H, Uno Y, Kawato M (1995) Internal representations of the motor apparatus: Implications from generalization in visuomotor learning. Journal of Experimental Psychology: Human Perception and Performance 21: 1174-1198.

Kitazawa S, Kohno T, Uka T (1995) Effects of delayed visual information on the rate and amount of prism adaptation in the human. J Neurosci 15: 7644-7652.

Krakauer JW, Pine ZM, Ghilardi MF, Ghez C (2000) Learning of visuomotor transformations for vectorial planning of reaching trajectories. J Neurosci 20: 89168924.

Krakauer JW, Ghilardi MF, Mentis M, Barnes A, Veytsman M, Eidelberg D, Ghez C (2004) Differential Cortical and Subcortical Activations in Learning Rotations and Gains for Reaching: A PET Study. J Neurophysiol 91: 924-933.

Magescas F, Prablanc C (2006) A joint-centred model accounts for movement curvature and spatial variability. Neurosci Lett 403: 114-118.

Magescas F, Urquizar C, Prablanc C (2009) Two modes of error processing in reaching. Exp Brain Res 193: 337-350.

Martin TA, Keating JG, Goodkin HP, Bastian AJ, Thach WT (1996) Throwing while looking through prisms: I. Focal olivocerebellar lesions impair adaptation. Brain 119: 1183-1198.

Mattar AAG, Ostry DJ (2007) Modifiability of Generalization in Dynamics Learning. J Neurophysiol 98: 3321-3329.

Mattar AAG, Ostry DJ (2010) Generalization of Dynamics Learning Across Changes in Movement Amplitude. J Neurophysiol 104: 426-438.

Mazzoni P, Krakauer JW (2006) An Implicit Plan Overrides an Explicit Strategy during Visuomotor Adaptation. J Neurosci 26: 3642-3645.

Paz R, Boraud T, Natan C, Bergman H, Vaadia E (2003) Preparatory activity in motor cortex reflects learning of local visuomotor skills. Nat Neurosci 6: 882-890.

Pelisson D, Prablanc C, Goodale MA, Jeannerod M (1986) Visual control of reaching movements without vision of the limb. II. Evidence of fast unconscious processes correcting the trajectory of the hand to the final position of a double-step stimulus. Exp Brain Res 62: 303-311.

Pine ZM, Krakauer JW, Gordon J, Ghez C (1996) Learning of scaling factors and reference axes for reaching movements. Neuroreport 7: 2357-2361.

Prablanc C, Martin O (1992) Automatic control during hand reaching at undetected two-dimensional target displacements. J Neurophysiol 67: 455-469.

Redding GM, Wallace B (2006) Generalization of prism adaptation. J Exp Psychol Hum Percept Perform 32: 1006-1022. 
Shadmehr R, Mussa-Ivaldi FA (1994) Adaptive representation of dynamics during learning of a motor task. J Neurosci 14: 3208-3224.

Shadmehr R, Krakauer J (2008) A computational neuroanatomy for motor control. Exp Brain Res 185: 359-381.

Shadmehr R, Wise SP (2005) The computational neurobiology of reaching and pointing: A foundation for motor learning. Cambridge, MA: The MIT Press.

Simani MC, McGuire LM, Sabes PN (2007) Visual-shift adaptation is composed of separable sensory and task-dependent effects. J Neurophysiol 98: 2827-2841.

Tanaka H, Sejnowski TJ, Krakauer JW (2009) Adaptation to visuomotor rotation through interaction between posterior parietal and motor cortical areas. J Neurophysiol 90834.

Thoroughman KA, Shadmehr R (1999) Electromyographic correlates of learning an internal model of reaching movements. J Neurosci 19: 8573-8588.

Thoroughman KA, Taylor JA (2005) Rapid Reshaping of Human Motor Generalization. J Neurosci 25: 8948-8953.

Tseng YW, Diedrichsen J, Krakauer JW, Shadmehr R, Bastian AJ (2007) Sensory prediction errors drive cerebellum-dependent adaptation of reaching. J Neurophysiol 98: 54-62.

Vetter P, Goodbody SJ, Wolpert DM (1999) Evidence for an eye-centered spherical representation of the visuomotor map. J Neurophysiol 81: 935-939.

Wise SP, Moody SL, Blomstrom KJ, Mitz AR (1998) Changes in motor cortical activity during visuomotor adaptation. Exp Brain Res 121: 285-299. 


\section{Original Manuscripts}

1. Gail A, Klaes C, and Westendorff S (2009). Implementation of Spatial Transformation Rules for Goal-Directed Reaching via Gain Modulation in Monkey Parietal and Premotor Cortex. J Neurosci 29: 9490-9499

2. Westendorff S, Klaes C, Gail A (2010) The Cortical Timeline for Deciding on Reach Motor Goals. J Neurosci 30: 5426-5436.

3. Klaes C, Westendorff S and Gail A (2011) Choosing Goals, Not Rules: Deciding among Rule-Based Action Plans. Neuron 70: 536-548.

4. Westendorff S and Gail A (2011) What is 'anti' about anti-reaches? - Reference frames selectively affect reaction times and endpoint-variability. Exp Brain Res 208: 287-296.

5. Westendorff S, Kuang S, Taghizadeh B, Donchin O, Gail A; Generalization of reach adaptation in a target jump paradigm; in preparation.

Author's contribution:

1. A.G., C.K. and S.W. designed the experiment, did the analysis and wrote the manuscript. C.K. and S.W. collected the data. All authors discussed the results and commented on the manuscript at all stages.

2. S.W., C.K. and A.G. designed the experiment. S.W. and C.K. collected the data. S.W. did the analysis and wrote the manuscript; A.G. edited the manuscript. All authors discussed the results and commented on the manuscript at all stages.

3. C.K., S.W., and A.G. designed the experiment. C.K., S.C. and S.W. collected the data. C.K. did the analysis and wrote the manuscript; A.G. edited the manuscript. All authors discussed the results and commented on the manuscript at all stages.

4. S.W. and A.G. designed the experiment. S.W. collected the data, did the analysis and wrote the manuscript. A.G. edited the manuscript. Both authors discussed the results and commented on the manuscript at all stages.

5. S.W. and A.G. designed the experiment. S.W. and B.T. collected the data. S.W. did the analysis and wrote the manuscript. A.G. and O.D. edited the manuscript. All authors discussed the results and commented on the manuscript at all stages. 


\section{Summary}

Every day we execute hundreds of reaches. Those reaches are done in different situations and driven by different purposes. This $\mathrm{PhD}$ thesis investigates how the neuronal representation and behavioral parameter of reach plans might be influenced by those different contexts. One electrophysiological study in monkeys showed that spatial tuning properties of neurons, that encode the reach plan of the monkey, are gain-modulated in their spatial tuning by a current valid task rule. We suggest that this gain modulation serves as a mechanism to flexibly remap spatial activity to achieve representations of the reach goal. Differences in the strength of this modulation between the investigated brain areas, PMd and PRR, suggest different functionalities for those areas within the cortical circuit for reach planning. The second electrophysiology study further investigated properties of this network by comparing latencies for the emergence of tuning for the reach goal. It rebuts a strict feed-forward network in which PRR first send reach goal information to PMd but rather suggests that this information is fed back from PMd to PRR. We interpret reach goal activity in PRR to be contingent on the projections from PMd and interareal latency differences as being dependent on visuospatial remapping processes in PRR. Furthermore task rules influenced the latencies within an area such that tuning for the reach goal was accomplished earlier if a spatial cue directly indicated a reach goal compared to situations in which the reach goal had to be spatially inferred. However, even though we suggest that PRR inherits reach goal activity from PMd we can not rule out an alternative interpretation in which a third brain region provides the input to PMd and PRR in parallel. To distinguish between those possibilities further studies could for example inactivate PMd. If an inactivation abolishes reach goal tuning in PRR that would provide strong evidence that the reach goal tuning in PRR is indeed contingent on the input from PMd.

To plan goal directed reaches it is important to know about the spatial position of the target object as well as about the position of the hand. This spatial information always has to be coded with respect to a reference point. A psychophysical study showed that overt behavioral parameters of the reach, as reaction time, movement time and reach endpoint variability, are influenced in a different way by reference frames. Reaction times, which are closer to the planning process, are affected by an eye- as well as a hand-centered reference frame. Movement times in contrast are influenced only by a 
hand-reference frame and endpoint variability only by an eye-reference frame. Those different reference frames might reflect the influences of different brain areas, which code target information in eye-, hand- or intermediate reference frames.

Changes in our environment might influence our reach precision. Motor adaptation is a mechanism to deal with those changes and re-gain former precision. In a psychophysical experiment, subjects adapted to an unconscious target displacement. For an angular displacement the adaptation effect transfers to directional neighboring target positions in such a way that the error vector just scales but does not rotate with different target directions. We interpret this result as in interdependent adaptation of direction and amplitude. The adaptation effect decreases with increasing distance from the adapted target. The decrease followed an asymmetric pattern. The adaptation effect was larger in the direction of the previous target displacement. The results of this study can be taken as psychophysical basis to investigate changes in neuronal activity under motor adaptation. Previous electrophysiological studies on reach adaptation focused mainly on the motor and premotor cortex (Paz et al., 2003; Paz et al., 2005; Mandelblat-Cerf et al., 2009; Arce et al., 2010a). Little is known about the influence of adaptation on response properties of neurons in the parietal cortex, even though an involvement of parietal cortex for reach adaptation had been shown (Clower et al., 1996; Diedrichsen et al., 2005). There are theoretical models (Donchin et al., 2003; Thoroughman and Taylor, 2005; Shadmehr and Krakauer, 2008; Tanaka et al., 2009) which provide suggestions for mechanisms underlying reach adaptation, which also include the parietal cortex. Further studies investigating changes in spatial tuning properties of neurons in brain areas of the frontoparietal reach network could test predictions of these theoretical models.

Taken together the results of the studies within this thesis show that a given context in which a reach is executed can influence how the plan for this movement is cortically represented. They help for a more detailed understanding of the cortical network underlying reaching behavior. In future the understanding of interactions of the given context with spatial constraints for goal directed movements might help for the further development of neuroprostheses. The idea of such devices is that cortical signals representing movement plan are used to program prostheses that can replace lost limbs. Next to technical challenges it would be important to understand how those cortical signals are influenced by the given situation in which the movement is 
executed. To successfully decode a movement plan and make it available for the prostheses one would have to take such factor into account. 


\section{Reference List}

Arce F, Novick I, Mandelblat-Cerf Y, Israel Z, Ghez C, Vaadia E (2010a) Combined Adaptiveness of Specific Motor Cortical Ensembles Underlies Learning. J Neurosci 30: 5415-5425.

Arce F, Novick I, Mandelblat-Cerf Y, Vaadia E (2010b) Neuronal Correlates of Memory Formation in Motor Cortex after Adaptation to Force Field. J Neurosci 30: 9189-9198.

Asaad WF, Rainer G, Miller EK (1998) Neural activity in the primate prefrontal cortex during associative learning. Neuron 21: 1399-1407.

Baizer JS, Kralj-Hans I, Glickstein M (1999) Cerebellar lesions and prism adaptation in macaque monkeys. J Neurophysiol 81: 1960-1965.

Baraduc P, Wolpert DM (2002) Adaptation to a visuomotor shift depends on the starting posture. J Neurophysiol 88: 973-981.

Barbas H, Pandya DN (1987) Architecture and frontal cortical connections of the premotor cortex (area 6) in the rhesus monkey. The Journal of Comparative Neurology 256: 211-228.

Batista AP, Buneo CA, Snyder LH, Andersen RA (1999) Reach plans in eye-centered coordinates. Science 285: 257-260.

Batista AP, Andersen RA (2001) The Parietal Reach Region Codes the Next Planned Movement in a Sequential Reach Task. J Neurophysiol 85: 539-544.

Battaglia-Mayer A, Caminiti R, Lacquaniti F, Zago M (2003) Multiple levels of representation of reaching in the parieto-frontal network. Cereb Cortex 13: 10091022.

Baumann MA, Fluet MC, Scherberger H (2009) Context-specific grasp movement representation in the macaque anterior intraparietal area. J Neurosci 29: 6436-6448.

Beurze SM, Van Pelt S, Medendorp WP (2006) Behavioral Reference Frames for Planning Human Reaching Movements. J Neurophysiol 96: 352-362.

Blatt GJ, Andersen RA, Stoner GR (1990) Visual receptive field organization and cortico-cortical connections of the lateral intraparietal area (area LIP) in the macaque. J Comp Neurol 299: 421-445.

Bock O, Eckmiller R (1986) Goal-Directed Arm Movements in Absence of Visual Guidance - Evidence for Amplitude Rather Than Position Control. Exp Brain Res 62: 451-458.

Bock O (1992) Adaptation of aimed arm movements to sensorimotor discordance: evidence for direction-independent gain control. Behavioural Brain Research 51: 4150 . 
Boettiger CA, D'Esposito M (2005) Frontal Networks for Learning and Executing Arbitrary Stimulus-Response Associations. J Neurosci 25: 2723-2732.

Boussaoud D, Wise SP (1993) Primate Frontal-Cortex - Effects of Stimulus and Movement. Exp Brain Res 95: 28-40.

Brozovic M, Gail A, Andersen RA (2007) Gain Mechanisms for Contextually Guided Visuomotor Transformations. J Neurosci 27: 10588-10596.

Bruce CJ, Goldberg ME (1985) Primate frontal eye fields. I. Single neurons discharging before saccades. J Neurophysiol 53: 603-635.

Bruce CJ, Goldberg ME, Bushnell MC, Stanton GB (1985) Primate frontal eye fields. II. Physiological and anatomical correlates of electrically evoked eye movements. J Neurophysiol 54: 714-734.

Burnod Y, Baraduc P, Battaglia-Mayer A, Guigon E, Koechlin E, Ferraina S, Lacquaniti F, Caminiti R (1999) Parieto-frontal coding of reaching: an integrated framework. Exp Brain Res 129: 325-346.

Calton JL, Dickinson AR, Snyder LH (2002) Non-spatial, motor-specific activation in posterior parietal cortex. Nat Neurosci 5: 580-588.

Caminiti R, Genovesio A, Marconi B, Mayer AB, Onorati P, Ferraina S, Mitsuda T, Giannetti S, Squatrito S, Maioli MG, Molinari M (1999) Early coding of reaching: frontal and parietal association connections of parieto-occipital cortex. Eur J Neurosci 11: 3339-3345.

Chang SWC, Snyder LH (2010) Idiosyncratic and systematic aspects of spatial representations in the macaque parietal cortex. PNAS 107: 7951-7956.

Clower DM, Hoffman JM, Votaw JR, Faber TL, Woods RP, Alexander GE (1996) Role of posterior parietal cortex in the recalibration of visually guided reaching. Nature 383: 618-621.

Clower DM, Boussaoud D (2000) Selective Use of Perceptual Recalibration Versus Visuomotor Skill Acquisition. J Neurophysiol 84: 2703-2708.

Colby CL, Gattass R, Olson CR, Gross CG (1988) Topographical organization of cortical afferents to extrastriate visual area PO in the macaque: a dual tracer study. $\mathrm{J}$ Comp Neurol 269: 392-413.

Colby CL, Goldberg ME (1999) Space and attention in parietal cortex. Annu Rev Neurosci 22: 319-349.

Connolly JD, Andersen RA, Goodale MA (2003) FMRI evidence for a 'parietal reach region' in the human brain. Exp Brain Res 153: 140-145.

Connolly JD, Goodale MA, Desouza JFX, Menon RS, Vilis T (2000) A Comparison of Frontoparietal fMRI Activation During Anti-Saccades and Anti-Pointing. J Neurophysiol 84: 1645-1655. 
Crammond DJ, Kalaska JF (1994) Modulation of preparatory neuronal activity in dorsal premotor cortex due to stimulus-response compatibility. J Neurophysiol 71: 1281-1284.

Desmurget M, Pelisson D, Urquizar C, Prablanc C, Alexander GE, Grafton ST (1998) Functional anatomy of saccadic adaptation in humans. Nat Neurosci 1: 524-528.

Diedrichsen J, Hashambhoy Y, Rane T, Shadmehr R (2005) Neural Correlates of Reach Errors. J Neurosci 25: 9919-9931.

Donchin O, Francis JT, Shadmehr R (2003) Quantifying generalization from trial-bytrial behavior of adaptive systems that learn with basis functions: Theory and experiments in human motor control. J Neurosci 23: 9032-9045.

Everling S, Desouza JFX (2005) Rule-dependent Activity for Prosaccades and Antisaccades in the Primate Prefrontal Cortex. J Cogn Neurosci 17: 1483-1496.

Gail A, Andersen RA (2006) Neural Dynamics in Monkey Parietal Reach Region Reflect Context-Specific Sensorimotor Transformations. J Neurosci 26: 9376-9384.

Gail A, Klaes C, Westendorff S (2009) Implementation of Spatial Transformation Rules for Goal-Directed Reaching via Gain Modulation in Monkey Parietal and Premotor Cortex. J Neurosci 29: 9490-9499.

Gnadt JW, Andersen RA (1988) Memory Related Motor Planning Activity in Posterior Parietal Cortex of Macaque. Exp Brain Res 70: 216-220.

Godschalk M, Lemon RN, Nijs HG, Kuypers HG (1981) Behaviour of neurons in monkey peri-arcuate and precentral cortex before and during visually guided arm and hand movements. Exp Brain Res 44: 113-116.

Goodale MA, Milner AD (1992) Separate visual pathways for perception and action. Trends Neurosci 15: 20-25.

Grafton ST, Mazziotta JC, Woods RP, Phelps ME (1992) Human functional anatomy of visually guided finger movements. Brain 115: 565-587.

Hanakawa T, Honda M, Zito G, Dimyan MA, Hallett M (2006) Brain activity during visuomotor behavior triggered by arbitrary and spatially constrained cues: an fMRI study in humans. Exp Brain Res 172: 275-282.

Henriques DYP, Klier EM, Smith MA, Lowy D, Crawford JD (1998) Gaze-centered remapping of remembered visual space in an open-loop pointing task. J Neurosci 18: 1583-1594.

Hoshi E, Shima K, Tanji J (1998) Task-dependent selectivity of movement-related neuronal activity in the primate prefrontal cortex. J Neurophysiol 80: 3392-3397.

Imamizu H, Uno Y, Kawato M (1995) Internal representations of the motor apparatus: Implications from generalization in visuomotor learning. Journal of Experimental Psychology: Human Perception and Performance 21: 1174-1198. 
Johnson PB, Ferraina S, Bianchi L, Caminiti R (1996) Cortical networks for visual reaching: Physiological and anatomical organization of frontal and parietal lobe arm regions. Cereb Cortex 6: 102-119.

Johnston K, Everling S (2006) Neural Activity in Monkey Prefrontal Cortex Is Modulated by Task Context and Behavioral Instruction during Delayed-Match-toSample and Conditional Prosaccade-Antisaccade Tasks. J Cogn Neurosci 18: 749765.

Jones EG, Powell TP (1970) An anatomical study of converging sensory pathways within the cerebral cortex of the monkey. Brain 93: 793-820.

Kitazawa S, Kohno T, Uka T (1995) Effects of delayed visual information on the rate and amount of prism adaptation in the human. J Neurosci 15: 7644-7652.

Krakauer JW, Pine ZM, Ghilardi MF, Ghez C (2000) Learning of visuomotor transformations for vectorial planning of reaching trajectories. J Neurosci 20: 89168924.

Kurata K (1989) Distribution of neurons with set- and movement-related activity before hand and foot movements in the premotor cortex of rhesus monkeys. Exp Brain Res 77: 245-256.

Kurata K (1993) Premotor cortex of monkeys: set- and movement-related activity reflecting amplitude and direction of wrist movements. J Neurophysiol 69: 187-200.

Kurata K, Hoshi E (1999) Reacquisition deficits in prism adaptation after muscimol microinjection into the ventral premotor cortex of monkeys. J Neurophysiol 81: 19271938.

Kurata K (1991) Corticocortical inputs to the dorsal and ventral aspects of the premotor cortex of macaque monkeys. Neuroscience Research 12: 263-280.

Li CS, Padoa-Schioppa C, Bizzi E (2001) Neuronal correlates of motor performance and motor learning in the primary motor cortex of monkeys adapting to an external force field. Neuron 30: 593-607.

Lu MT, Preston JB, Strick PL (1994) Interconnections between the prefrontal cortex and the premotor areas in the frontal lobe. The Journal of Comparative Neurology 341: 375-392.

MacAvoy MG, Gottlieb JP, Bruce CJ (1991) Smooth-Pursuit Eye Movement Representation in the Primate Frontal Eye Field. Cereb Cortex 1: 95-102.

Mandelblat-Cerf Y, Paz R, Vaadia E (2009) Trial-to-Trial Variability of Single Cells in Motor Cortices Is Dynamically Modified during Visuomotor Adaptation. J Neurosci 29: 15053-15062.

Marconi B, Genovesio A, Battaglia-Mayer A, Ferraina S, Squatrito S, Molinari M, Lacquaniti F, Caminiti R (2001) Eye-hand coordination during reaching. I. Anatomical relationships between parietal and frontal cortex. Cereb Cortex 11: 513527. 
Martin TA, Keating JG, Goodkin HP, Bastian AJ, Thach WT (1996) Throwing while looking through prisms: I. Focal olivocerebellar lesions impair adaptation. Brain 119: 1183-1198.

Mattar AAG, Ostry DJ (2007) Modifiability of Generalization in Dynamics Learning. J Neurophysiol 98: 3321-3329.

Mattar AAG, Ostry DJ (2010) Generalization of Dynamics Learning Across Changes in Movement Amplitude. J Neurophysiol 104: 426-438.

Mazzoni P, Bracewell RM, Barash S, Andersen RA (1996) Motor intention activity in the macaque's lateral intraparietal area. I. Dissociation of motor plan from sensory memory. J Neurophysiol 76: 1439-1456.

Mazzoni P, Krakauer JW (2006) An Implicit Plan Overrides an Explicit Strategy during Visuomotor Adaptation. J Neurosci 26: 3642-3645.

McGuire LMM, Sabes PN (2009) Sensory transformations and the use of multiple reference frames for reach planning. Nat Neurosci 12: 1056-1061.

Medendorp WP, Goltz HC, Vilis T (2005) Remapping the Remembered Target Location for Anti-Saccades in Human Posterior Parietal Cortex. J Neurophysiol 94: 734-740.

Mountcastle VB, Lynch JC, Georgopoulos AP, Sakata H, Acuna C (1975) Posterior parietal association cortex of the monkey: command functions for operations within extrapersonal space. J Neurophysiol 38: 871-908.

Norris SA, Greger BE, Martin TA, Thach WT (2001) Prism adaptation of reaching is dependent on the type of visual feedback of hand and target position. Brain Res 905: 207-219.

Pandya DN, Kuypers HGJM (1969) Cortico-cortical connections in the rhesus monkey. Brain Res 13: 13-36.

Paz R, Boraud T, Natan C, Bergman H, Vaadia E (2003) Preparatory activity in motor cortex reflects learning of local visuomotor skills. Nat Neurosci 6: 882-890.

Paz R, Natan C, Boraud T, Bergman H, Vaadia E (2005) Emerging Patterns of Neuronal Responses in Supplementary and Primary Motor Areas during Sensorimotor Adaptation. J Neurosci 25: 10941-10951.

Perenin MT, Vighetto A (1988) Optic ataxia: A specific disruption in visuomotor mechanisms. Brain 111: 643-674.

Petrides M, Pandya DN (1999) Dorsolateral prefrontal cortex: comparative cytoarchitectonic analysis in the human and the macaque brain and corticocortical connection patterns. Eur J Neurosci 11: 1011-1036.

Pine ZM, Krakauer JW, Gordon J, Ghez C (1996) Learning of scaling factors and reference axes for reaching movements. Neuroreport 7: 2357-2361. 
Rizzolatti G, Camarda R, Fogassi L, Gentilucci M, Luppino G, Matelli M (1988) Functional organization of inferior area 6 in the macaque monkey. II. Area F5 and the control of distal movements. Exp Brain Res 71: 491-507.

Rizzolatti G, Gentilucci M, Fogassi L, Luppino G, Matelli M, Ponzoni-Maggi S (1987) Neurons related to goal-directed motor acts in inferior area 6 of the macaque monkey. Exp Brain Res 67: 220-224.

Rizzolatti G, Luppino G (2001) The cortical motor system. Neuron 31: 889-901.

Rushworth MFS, Paus T, Sipila PK (2001) Attention Systems and the Organization of the Human Parietal Cortex. J Neurosci 21: 5262-5271.

Sakata H, Taira M (1994) Parietal control of hand action. Curr Opin Neurobiol 4: 847-856.

Shadmehr R, Krakauer J (2008) A computational neuroanatomy for motor control. Exp Brain Res 185: 359-381.

Snyder LH, Batista AP, Andersen RA (1997) Coding of intention in the posterior parietal cortex. Nature 386: 167-170.

Snyder LH, Batista AP, Andersen RA (2000) Intention-related activity in the posterior parietal cortex: a review. Vision Res 40: 1433-1441.

Sorrento GU, Henriques DYP (2008) Reference Frame Conversions for Repeated Arm Movements. J Neurophysiol 99: 2968-2984.

Stoet G, Snyder LH (2004) Single Neurons in Posterior Parietal Cortex of Monkeys Encode Cognitive Set. Neuron 42: 1003-1012.

Tanaka H, Sejnowski TJ, Krakauer JW (2009) Adaptation to visuomotor rotation through interaction between posterior parietal and motor cortical areas. J Neurophysiol 90834.

Tanne-Gariepy J, Rouiller EM, Boussaoud D (2002) Parietal inputs to dorsal versus ventral premotor areas in the macaque monkey: evidence for largely segregated visuomotor pathways. Exp Brain Res 145: 91-103.

Thoroughman KA, Taylor JA (2005) Rapid Reshaping of Human Motor Generalization. J Neurosci 25: 8948-8953.

Wallis JD, Anderson KC, Miller EK (2001) Single neurons in prefrontal cortex encode abstract rules. Nature 411: 953-956.

Wallis JD, Miller EK (2003) From Rule to Response: Neuronal Processes in the Premotor and Prefrontal Cortex. J Neurophysiol 90: 1790-1806.

Wang J, Sainburg RL (2005) Adaptation to Visuomotor Rotations Remaps Movement Vectors, Not Final Positions. J Neurosci 25: 4024-4030. 
Weiner MJ, Hallett M, Funkenstein HH (1983) Adaptation to lateral displacement of vision in patients with lesions of the central nervous system. Neurology 33: 766-772.

Weinrich M, Wise SP (1982) The premotor cortex of the monkey. J Neurosci 2: 13291345.

Weinrich M, Wise SP, Mauritz KH (1984) A Neurophysiological Study of the Premotor Cortex in the Rhesus Monkey. Brain 107: 385-414.

White IM, Wise SP (1999) Rule-dependent neuronal activity in the prefrontal cortex. Exp Brain Res 126: 315-335.

Wise SP, Boussaoud D, Johnson PB, Caminiti R (1997) Premotor and Parietal Cortex: Corticocortical Connectivity and Combinatorial Computations. Annu Rev Neurosci 20: $25-42$.

Wise SP, Mauritz KH (1985) Set-related neuronal activity in the premotor cortex of rhesus monkeys: effects of changes in motor set. Proc R Soc Lond B Biol Sci 223: 331-354.

Wise SP, Moody SL, Blomstrom KJ, Mitz AR (1998) Changes in motor cortical activity during visuomotor adaptation. Exp Brain Res 121: 285-299.

Wise SP, Weinrich M, Mauritz KH (1986) Movement-related activity in the premotor cortex of rhesus macaques. Prog Brain Res 64: 117-131.

Zipser D, Andersen RA (1988) A back-propagation programmed network that simulates response properties of a subset of posterior parietal neurons. Nature 331: 679-684. 


\section{Stephanie Westendorff}

Affiliation German Primate Center, Sensorimotor Group, Kellnerweg 4, 37077 Göttingen

Email: $\quad$ swesten@gwdg.de

Phone: $\quad+49(0) 5513851342$

Education:

2010 Dr. rer. nat. in System Neuroscience, Title: 'Neuronal mechanisms of the adaptation of conditional visuomotor behavior'. Graduated "Summa cum laude"

Biology Diploma, Title: 'Topography of visual structures in the pretectum for slow eye movements in a foveate fish (Serranus cabrilla)', Dept. of General Zoology and Neurobiology, RuhrUniversity Bochum.

2000-2005 Study of biology at the Ruhr-University, Bochum.

2000 Graduated from highschool, Essen, Germany.

\section{Professional Experience:}

2010- Postdoctoral researcher in Dr. Alexander Gail's laboratory, Sensorimotor Group, German Primate Center, Göttingen.

2006-2010 Research associate in Dr. Alexander Gail's laboratory, Sensorimotor Group, German Primate Center, Göttingen.

2006

Scientific assistant, Dept. of General Zoology and Neurobiology, Ruhr-University Bochum.

2006

Internship with Prof. Dr. Nikolaus Troje, Biomotion Lab, Queens University, Kingston, Ontario, Canada.

Grants: 
Courses:

2010

CSHL summer course: 'Computational Neuroscience: Vision', Cold Spring Harbor, USA

2009

PENS/Hertie Winter School: 'Structure and Function of Neural Circuits', Obergurgl, Austria

2008

Computational Workshop: 'Cue combination - Unifying

perceptual theory', Rauischholzhausen, Germany

Professional Memberships:

Society for Neuroscience since 2008

$\underline{\text { Organized Meetings: }}$

'Neuroprosthetics: from sensorimotor research to applications', PhD-Symposium 2010 of the Göttingen Graduate School for Neurosciences and Molecular Biosciences (GGNB)

Teaching experience:

2007-2011 Organization of the Girls Day in the lab

$2010 \quad$ Supervision of a practical project (Patric Pelzer)

2009 Supervision of a practical project (Angelika Schulz)

2009 Supervision of a labrotation project (Roman Stilling)

2009 Supervision of a practical project (Constanze Lenschow)

2009 Supervision of a diploma thesis (Mark Kaltenhäuser)

2008 Supervision of a labrotation project (Sadeem Jawahr) 